NBS

Publi-

cations

Reference

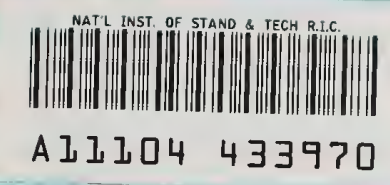

NBSIR 82.2472

Migration of Low Molecular Weight Additives in Polyolefins and Copolymers

U.S. DEPARTMENT OF COMMERCE

National Bureau of Standards

- Center for Materials Suience

Polymer Science and Standards Civision

Washington, DC $20: 34$

Final Project Report

Issued March 1982

$32-2472$

1982

Prepared for

Buraau of Foods

Food and Drug Administration

Washington, DC 20201 


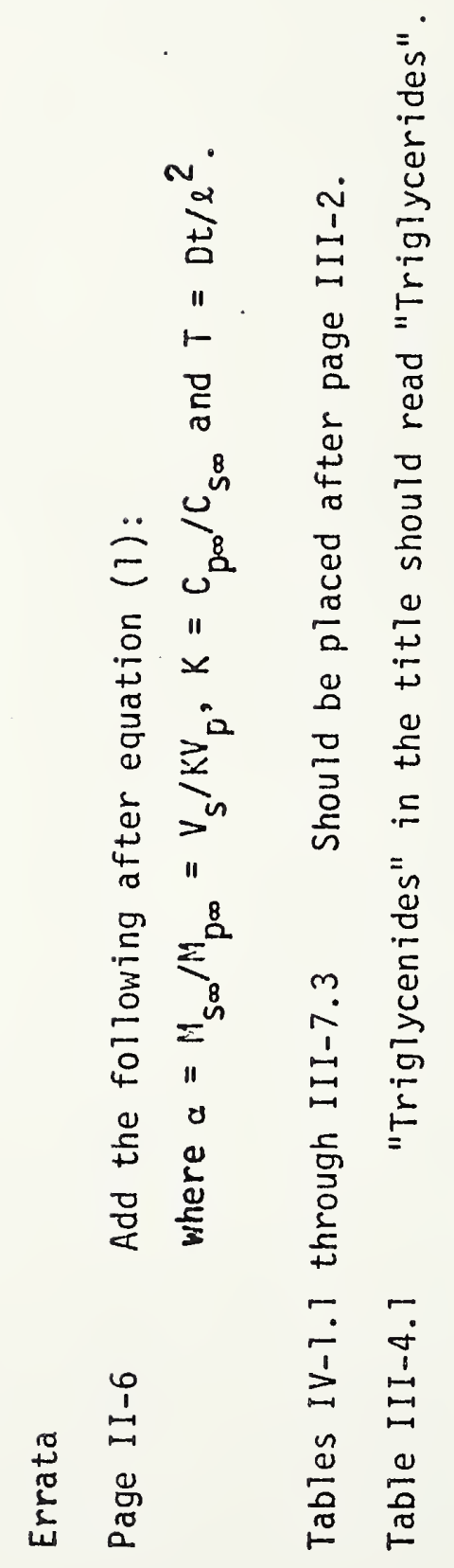




\section{MIGRATION OF LOW MOLECULAR WEIGHT ADDITIVES IN POLYOLEFINS AND COPOLYMERS}

S. S. Chang, G. A. Senich and L. E. Smith

U.S. DEPARTMENT OF COMIVIERCE National Bureau of Standarcis Center for Materials Science Polymer Science and Standards Division Washington, DC 20234

Final Project Report

Issued March 1982

Prepared for

Bureau of Foods

Food and Drug Administration

Washington, DC 20201

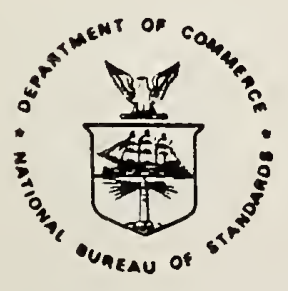

U.S. DEPARTMENT OF COMMERCE, Malcolm Baldrige, Secretary NATIONAL BUREAU OF STANDARDS. Ernest Ambler, Director 



\section{Abstract}

Food fackaging is an essential part of modern life. Any substances that migrate from packaging material into foods are viewed as indirect food additives. In addition to toxicological knowledge, it is important to know the amount of such indirect food additives expected to be present in the food during storage and processing. This program, sponsored by the Bureau of Foods of the Food and Drug Administration, is designed to provide theoretical models, a reliable data base and methodology for studying migration phenomena and can provide reasonable worst-case estimates for the concentrations of the indirect additives in foods. In this final report, we present the results of approximately 250 completed migration experiments based on radiotracer techniques on the migration of low molecular weight hydrocarbons and antioxidants from polyethylene, polypropylene, and ethylene-vinyl acetate copolymers. Results of a study for the determination of relative diffusion coefficients of severai probe molecules in the polyethylene melt by inverse gas chromatography are also presented. Based on these studies, ethanol appears to be a far more reasonable food-oil stimulating solvent than $n$-heptane. Other pure or mixed triglycerides may also be considered as food-oil simulating solvents, however they may pose the same analytical difficulties associated with the use of the food oils themselves.

Keywords: antioxidants, diffusion, ethylene-vinyl acetate copolymers, food additives, food packaging, inverse gas chromatography, migration, oligomers, polyethylene, polypropylene, radiotracer. 

Migration of Low Molecular Weight Additives in Polyolefins and Their Copolymers

1. Introduction

II. Experimental Details of Extraction "Experiments

Materials

Sample Plaque Preparation .

Experimental Methods

Run Designation

Estimation of Diffusion Coefficients

III. Results of Polyolefin Extraction Experiments

IV. Loss of BHT from Polymers Stored at Ambient Temperature

V. Migration of BHT from Ethylene-Vinyl Acetate Copolymer

VI. IGC Determinations of the Relative Diffusion Coefficients of Several Probes in Linear Polyethylene

A Review of Pertinent Theory for IGC

Absolute Diffusivity Determinations

Summary of Previous Results for LPE-Octadecane

Experimental Details

Relative Diffusivity Determinations

Future Results

VII. A Simplified Migration Decision Tree

Load Limited Migration.

Partition Limited Migration

Diffusion Limited Migration

VIII. Simulating Solvents

IX. Summary of Conclusions

X. Appendix: Numerical Data for Migration of Low Molecular Weight Additives from Polyolefins and Copolymers 


\section{List of Figures}

IV-1 BHT Content as a Function of Time

V-1.1 Migration of BHT from E-5\% VA Copolymer at $30^{\circ} \mathrm{C}$

$V-1.2$ Migration of BHT from E-5\% VA Copolymer at $60^{\circ} \mathrm{C}$

V-1.3 Migration of BHT from E-13\% VA Copolymer at $30^{\circ} \mathrm{C}$

V-1.4 Migration of BHT from E-13\% VA Copolymer at $60^{\circ} \mathrm{C}$

V-2.1 Migration of BHT from E-VA Copolymers into $n$-Heptane

V-2.2 Migration of BHT from E-VA Copolymers into Corn 011

V-2.3 Migration of BHT from E-VA Copolymers into Ethanol

V-2.4 Migration of BHT from E-VA Copolymers into 95\% Ethanoi

VI-1 Elution Chromatogram Schematic

VI-2 van Deemter Plot

VI-3 Illustration of Polymer Phase Geometries within an IGC Column

VI-4 Typical Recorder Output of a Probe Peak

VI-5 High Speed Chart Record of Five Determinations of $t_{m}$ with Methane

VI-6 Typical High Speed Chart Record of a Probe Peak and First Time Derivative

VI-7 Plot of $H^{-1} / u_{0}$ vs. $2 p_{0} /\left(p_{i}+p_{0}\right)$

VII-1 Flow Chart of Decision Tree

VIII-1 Comparison of Diffusion Coefficients in Pure Triglycerides and Corn 0il

VIII-2 Comparison of Diffusion Coefficients in Ethanol and Corn 0 il

VIII-3 Effect of Accelerating Solvent

VIII-4 Molecular Weight Distributions of Ethanol and n-Heptane Extracts of LPE

VIII-5 Molecular Weight Distributions of Ethanol and n-Heptane Extracts of BPE 


\section{List of Tables}

II-1 Characteristics of Polyolefin Samples

II-2 Characteristics of Ethylene-Vinyl Acetate Copolymers

II-3 Characteristics of Radioactive Tracers

II-4 Run Designation Coding--Solvent, Temperature, Method

Ii -5 Run Designation Coding--Sample

III-1.i Migration of $n$-Octadecane from Linear Polyethylene

.2 Migration of $n$-Octadecane to/from Linear Polyethylene

.3 Migration of $n-0 c t a d e c a n e$ from Branched Polyethylene

.4 Migration of $n$-Octadecane to/from Branched Polyethylene

.5 Migration of $n$-Octadecane from Polypropylene

III-2.1 Migration of n-Dotriacontane from Linear Polyethylene

.2 Migration of $n$-Dotriacontane from Branched Polyethylene

.3 Migration of $n$-Dotriacontane from Polypropylene

III-3.1 Migration of BHT from Linear Polyethylene

.2 Migration of BHT from Branched Polyethylene

.3 Migration of BHT from Polypropylene

III-4.? Migration of n-Octadecane into Triglycerides

.2 Migration of $n-0 c t a d e c a n e$ into Ethanol and $n-0 c t a n o l$

.3 Migration of $n-0 c t a d e c a n e$ into Ethanol/Water Mixture

.4 Migration of $n$-Octadecane into $n$-Heptane

.5 Migration of $n$-Octadecane into $n$-Octadecane

.6 Migration of n-Octadecane into Water

III-5.1 Migration of n-Dotriacontane into Triglycerides

.2 Migration of $n$-Dotriacontane into Ethanol, Ethanol/Water Mixture and n-Octanol

.3 Migration of $n$-Dotriacontane into $n$-Heptane

.4 Migration of $n$-Dotriacontane into Water 
III-5:1 Migration of BHT into Triglycerides

.2 Migration of BHT into Ethanol, Ethanol/Water Mixtures and n-Octanol

.3 Migration of BHT into n-Heptane

.4 Migration of BHT into Water

III-7.1 Activation Energies of Migration of $n$-Octadecane

.2 Activation Energies of Migration of $n$-Dotriacontane

.3 Activation Energies of Migration of BHT

IV ..? LOSS of BHT

V-1.1 Migration of BHT from Ethylene-5\% Vinyl Acetate Copolymer

.2 Migration of BHT from Ethylene-13\% Vinyl Acetate Copolymer

$V=2 \quad$ Diffusion Coefficients and Activation Energies of Migration of BHT from E-VA Copolymers

VI-1 Comparison of the C Coefficient Determined by Two Methods for LPE-Octadecane at $150^{\circ} \mathrm{C}$

- 2 Effective Layer Thicknesses for Columns with Differing Polymer Phase Geometry

- 3 Influence of Film Geometry on the Diffusion Coefficient of Octadecane in Linear Polyethylene at $150^{\circ} \mathrm{C}$

- 4 Effect of Thickness Distribution on Diffusivity in the Polymer Phase

- 5 Probe Employed in an IGC Study of Relative Diffusivity in LPE

- 6 Experimenta? Data for an IGC Diffusivity Study of Tridecane in LPE at $150^{\circ} \mathrm{C}$

- 7 Relative Diffusivity Results for Several Alkanes and BHT in LPE at $150^{\circ} \mathrm{C}$

VIII-1 Extraction of Polyethylenes by Solvents

- 2 Molecular Weight Distributions of Ethanol and n-Heptane Extractions of Poi yethylenes 


\section{Introduction}

As in the proceeding decades, most foods available today are sold in packaged forms rather than in bulk for reasons of preservation, convenience and ease of distribution. The Bureau of Foods of the Food and Drug Administration has regulatory responsibility for the use of packaging materials in contact with food. Any substances that migrate from the food packaging materials into foods are considered to be indirect food additives. The regulatory decisions on the types of packaging materials suitable for use with various types of foods are based on: the indirect food additives that can be expected to migrate from the package into the food, ones knowledge of toxicology and the expected amount of migration during storage and usage. Since it is impossible to test every conceivable combination of polymer-migrant-food, this program at the National Bureau of Standards, sponsored by the Food and Drug Administration, is aimed at providing sound technical suggestions and reliable data to the Food and Drug Administration, and recommending theoretical or empirical estimation and extrapolation methods to yield at least a reasonable worst-case estimates for regulatory purposes. However, this report represents only the technical opinions of the authors as representatives of the National Bureau of Standards and should not be taken in any way as containing regulatory decisions or recommendations of the Bureau of Foods.

A large number of kinetic experiments were performed involving the migration of radioactive ${ }^{14} \mathrm{C}$-labeled paraffinic oligomers ( $n$-octadecane and n-dotriacontane) and an antioxidant (butylated-hydroxytoluene) from two different polyethylenes (high density or linear poiyethylene and low density or branched polyethylene), isotactic polypropylene and two (ethylene-vinyl acetate) copolymers of relatively low vinyl acetate 
content into various solvents including a variety of triglycerides, alcohols, hydrocarbons, and water at temperatures from room temperature to $60^{\circ} \mathrm{C}$.

Among the correlations for various parameters, it was found that either the pure triglycerides (e.g. tributyrin and trioctanoin), mixed triglycerides, or anhydrous ethanol may successfully be employed as food-oil simulant in all cases studied. The degree of accelerating action of extracting migrants by $n$-heptane over that by the food oi?s is quantified.

Future works in the additive migration program should be mainly in the area of migration of additives from glassy polymers, where the diffusion coefficients are many orders of magnitude slower than those for the amorphous or semi-crystalline polymers above their glass transition temperatures.

Inverse gas chromatography (IGC) studies have been used to determine the relative diffusion coefficients of 01 igomers and antioxidants in polymers at temperatures above the glass transition region and in the supercooled-liquid or liquid phase. The determinations of the relative diffusion coefficients of several probe molecules in polyethylene are illustrated. 


\section{Experimental Details of Extraction Experiments}

All scheduled experiments on the migration of additives from polyolefins (polyethylenes and polypropylenes) are now completed. In this report we present a complete summary of the polyolefin work, most of which has been reported in previous NBSIRs [1-6] on this subject, therefore, other than necessary descriptions to make this report selfexplainatory, detailed discussions on each sub-category will not be repeated here.

As the copolymer work was started in mid-year and was just completed recently, rather detailed descriptions and discussions of it will be given in this report.

Migrations of low molecular weight additives moving from polymeric materiais into surrounding liquid media under well stirred conditions were measured in this laboratory for the following combinations. of base. polymer, migrant, migrant concentration, solvent and temperature:

$\begin{array}{ll}\text { Polymers } & \text { Linear Polyethylene (LPE), SRM } 1475 \\ & \text { Branched Polyethylene (BPE), SRM } 1476 \\ & \text { Polypropylene (PP) } \\ & \text { Ethylene-5\% Vinyl Acetate Copolymer } \\ & \text { Ethylene-13\% Vinyl Acetate Copolymer } \\ & \text { n-Octadecane, n- } \mathrm{C} 18 \mathrm{H}_{38} \\ \text { Migrants } & \mathrm{n} \text {-Dotriacontane, n- } \mathrm{C}_{32}{ }^{\mathrm{H}} 66 \\ & \text { BHT or 3,5-di-t-butyl-4-hydroxytoluene }\end{array}$

Migrant Con- From $30 \mathrm{ppm}$ to $10 \%$ centration

Solvents

Accelerating solvents: $n$-Heptane, $n$-Octadecane Fat and Dil Simulating Solvents: Corn 0il, Ethanol, n-Octanoi, Tributyrin, Trioctanoin, HB307 Aqueous Solvents: Water, Ethanol-Water Mixtures

Temperatures $24,30,40,60^{\circ} \mathrm{C}$

The migrants are radiolabeled by carbon-14. The amount of radioactivity is determined by liquid scintillation counting techniques with detection limit less than $10 \mathrm{pCi}$. 
Materials

The characteristics of the base polymers are listed in Table II-l and II-2 and those of the base radioactive-labeled migrants are 1 isted in Table II-3.

Samole Plaque Preparation

The following procedure for the mixing of additives in the polymer stock and the molding of the sample plaques was chosen. A large quantity of polyethylene powder stock was prepared from either the National Bureau of Standards-Standard Reference Material (NBS-SRM) 1475 or 1476 pellets by first dissolving it in hot toluene or xylene. Most of the polyethylene precipitates out upon cooling. The precipitate, together with the residue obtained by evaporating the solvent, was dried in a vacuum oven to remove the last trace of solvent.

A specific amount of labeled additive dissolved in a highty volatile solvent is mixed with a quantity of the polyethylene powder stock. The mixture, together with a number of glass beads which act as a ball

- mil?, is then evaporated to dryness in a rotary evaporator under reduced pressure at relatively low temperatures. Further drying is carried out in a yacuum oven.

The mixture is then compression molded in a hydraulic press operated at about $180^{\circ} \mathrm{C}$ for oligomers and $165^{\circ} \mathrm{C}$ for $\mathrm{BHT}$. Plaques of $125 \mathrm{~mm}$ x $125 \mathrm{~mm}$ or less are molded with brass or stainless steel shim stocks of appropriate thickness sandwiched between two sheets of Teflon or Teflon coated plates. Teflon surfaces are used to allow easy removal of the sample plaques without the use of, and subsequent contamination by, mold-release agents.

The isotactic polypropylene and (ethylene-vinyl acetate) copolymers were received in granular form from the suppliers. Therefore, they were used directly to form slurries with the migrants in solution as des- 
Special problems are encountered in the preparation of sample plaques with BHT as an additive. A much longer time is required to mix BHT with polymeric powder in a rotating flask with glass beads acting as a ball mill. Apparently BHT is relatively insoluble in the polymer even in the molten state, therefore, sample plaques made from such insufficiently mixed batches of powder mixtures yield autoradiographs of distinctively sharp regions of streaks and patches. Each molding at $185^{\circ} \mathrm{C}$ seems to "fix" an additional $15-20 \%$ of the available BHT, i.e. only $80 \%$ of the avialable BHT before the last molding may be extracted exhaus:tively even by $n$-heptane. Only $20 \%$ of original amount of BHT can be extracted from plaques remolded five times at $185^{\circ} \mathrm{C}$. The residual BHT is combined with the polymer. This residual radioactivity stays in the polymeric precipitate, even after the plaques were dissolved in toluene at high temperatures. However, molding of well mixed batches of BHT and polymeric powder at $165^{\circ} \mathrm{C}$ seems to result in rather uniformly distributed sample plaques with less than $5 \%$ of BHT being combined with the polymer. The final fraction extracted from the original loading, $M_{f} / M_{0}$, by $n-$ heptane, as listed in Tables in Section III, gives an indication of the unreacted fraction of BHT in those samples. Therefore the original loading $M_{0}$, is irrelevant and should be replaced by the remaining unreacted amount, $M_{f}$, for kinetic and partition calculations. There are further complications due to the volatility of BHT or its degraded components as detailed later in Section II.

\section{Experimental Methods}

Two extraction methods were used: (1) continuous extraction into a limited solvent volume and (2) discrete extraction into a simulated infinite solvent volume. 
In method (1) an extraction vial of $25 \mathrm{ml}$ volume with a Teflon valved cap is used. The 10 to $15 \mathrm{ml}$ of solvent, in the vial will only contact glass walls and Teflon surfaces during normal experimental processes. A silicone plug is situated above the valve. A small area of the silicone rubber, less than $1 \mathrm{~mm}$ in diameter which is used as a septum for the hypodermic needle, is exposed to the solvent vapor. The polymer sample was sometimes surrounded by a nichrome or stainless steel screen to prevent it from sticking to another sample or to the walls, if the sample has lower density than the solvent.

The total amount extracted, at time $t, M_{t}$, is the sum of the amount of migrant in the solution at time $t$ plus that were removed in previous al iquots,

$$
M_{t}=C_{s t} W_{s t}+\sum_{i=1}^{t-1} c_{s i} W_{a i}
$$

where $C_{s}, W_{s}$ and $W_{a}$ represent the concentration of the migrant, total weight of the solution (including that of the aliquot for the determination of $C_{S t}$ ) and the weight of the aliquot, respectively. At equilibrium the partition coefficient is estimated as the ratio of the concentrations in solvent and in polymer at equilibrium,

$$
k=\frac{C_{S \infty}}{C_{p \infty}}=\frac{C_{S \infty} W_{p}}{M_{0}-M_{\infty}}
$$

where $M_{0}$ is the amount of migrant originally present in the polymer of weight $W_{p}$, and $M_{\infty}$ is the total amount extracted at long times.

In method (2), the polymer sample is immersed in about $10 \mathrm{ml}$ of extracting solvent in a typical $20 \mathrm{ml}$ liquid scintillation counting vial. At specific times the sample is removed from the solvent, rinsed, and placed in another vial with fresh solvent to repeat the extraction process. The rinse is then combined with the previous extracting solvent. The total amount of migrant extracted at time $t$ is simply the sum 
of the migrant from all extracts:

$$
M_{t}=\sum_{i=1}^{t} M_{j}
$$

Method (1) is able to yield information about the equiitibrium partition coefficient at an infinite extraction time. However, this method suffers from the rigid requirements of knowing accurately the weight or volume ratio of aliquot to total solution and accounting for materials lost during the sampling process for material balance purposes. As the extraction time increases, there is only a very small change in the concentration of extracted material in the solution, whereas the weighing or ratio error may persist. Therefore, the results for method (1) at long times or at high degree of extraction will show considerable scatter.

Method (2) is much simpler in operation, but simulates a condition of migration into infinite media. It is relatively free from aforementioned experimental difficulties. However, it should oniy be used for convenience where the migrant is highly soluble in or miscible with the solvent and thus the results of method (1) and method (2) are indistinguishable. It can neither be used to generate equilibrium partition information nor migration kinetics for cases where the migrant is sparingly soluble in the solvent.

A method often used in the literature consists of repienishing the amount of solution aliquot removed for testing with the same amount of fresh solvent, to keep the volume of solvent and exposed surface area ratio the same throughout the experiment. This method is a hybrid of method (1) and method (2), and thus suffers from the same drawback:s as those of method (2).

For both methods (1) and (2) mentioned above, the extraction vials are shaken inside a temperature controlled aluminum block on a shaking 
table at a rate of about 200 reciprocations per minute.

In order to check the mass balance and variations in the migrant concentration, radioactivity of the residual low molecular weight species remaining in the polymeric sample is monitored by dissolving the sample in toluene at high temperatures after the extraction proceedure is ended. We have found that the single crystals or precipitates of polymer in the counting vial do not interfere with the counting efficiency beyond the normal uncertainty of the counting results. Experimental Run Designation

The numerical representation of experimental run designations, PPSSTTN, are assigned as follows:

1) Least significant $\operatorname{digit}(\boldsymbol{N})$--method of observation and repetition number.

2) Tens and Hundreds digits(TT)--temperature in ${ }^{\circ} \mathrm{C}$.

3) Thousands and Ten Thousands digits(SS)--solvent code.

4) Hundred Thousands and Millions digits(PP)--sample code.

This coding scheme is described in more detail in Table II-4 and II-5. Estimation of Diffusion Coefficient

One of the widely used solutions for the Fickian diffusion equations solved for the case of diffusion between a plane sheet $p$ of thickness $2 l$ and a stirred liquid s of finite volume $V_{S}$, is presented by $J$. Crank ${ }^{[7]}$

$$
\frac{M_{t}}{M_{\infty}}=1-\sum_{n=1}^{\infty} \frac{2 \alpha(1+\alpha)}{1+\alpha+\alpha^{2} q_{n}^{2}} \exp \left(-q_{n}^{2} T\right)
$$

The solution for the non-zero positive roots, $q_{n}$, of

$$
\tan q_{n}=-\alpha q_{n}
$$

lies between $n \Pi$ when $\alpha=0$ and $(n-1 / 2) \Pi$ when $\alpha=\infty$. At $\alpha<1$,

$$
q_{n} \sim n /(1+\alpha)
$$

For other values of $\alpha$,

$$
q_{n} \sim[n-\alpha / 2(1+\alpha)] \pi
$$


as

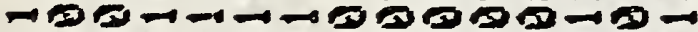

x 4 a

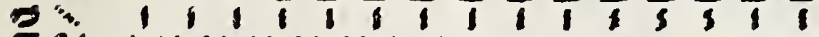

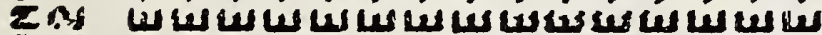

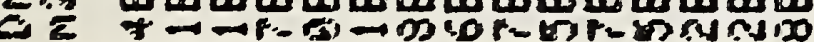

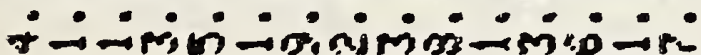

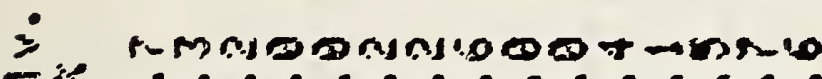

2.

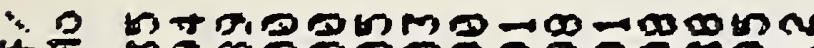

$4=$ mo.sh

2

نे

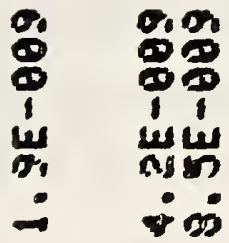

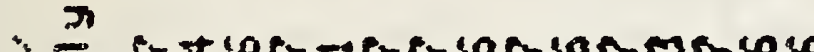

a $\square ? \cdots$

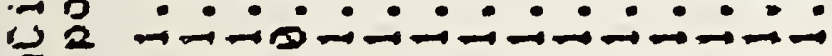

2

a) $\infty \mathrm{s}$

- iris

or ars

$\therefore \quad 0$

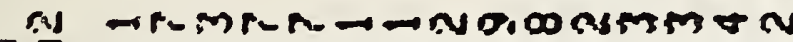

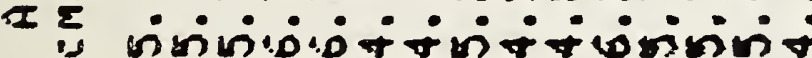

$\underset{-1}{0}$

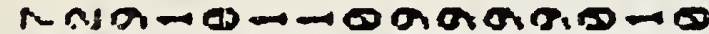

LOH.

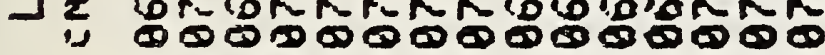

வ்

0 nis
0 nim

m $\infty 0$

(n)

- No

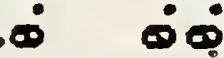

- -nitgonnomaradog

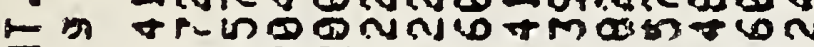

$\rightarrow-\rightarrow \mathrm{N} \times-\rightarrow-\infty \rightarrow-1$

ம்

$\rightarrow \infty$

o m

-

क $\quad \circ$

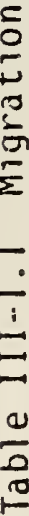

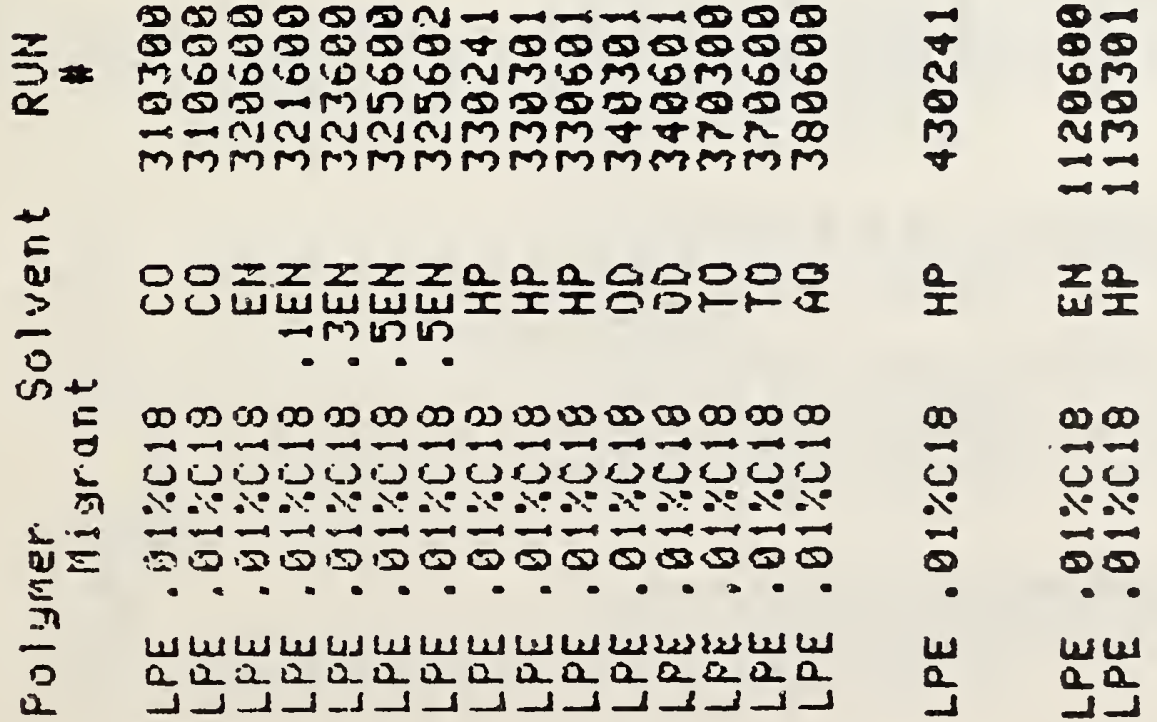




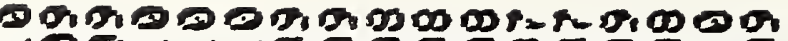

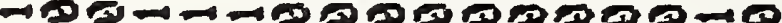

x 4 2

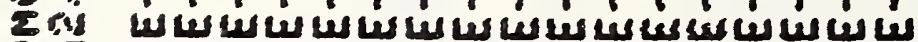

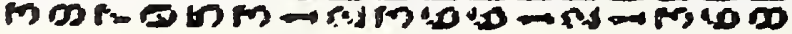
$\therefore$ -

$\frac{2}{n}$

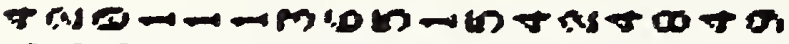

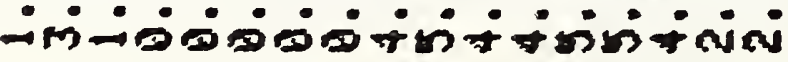

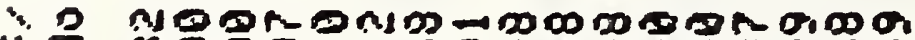

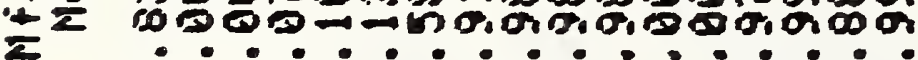

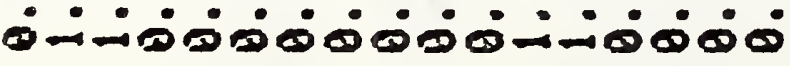

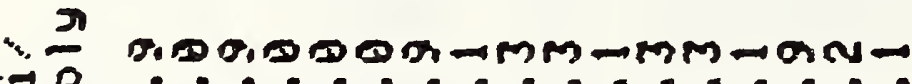

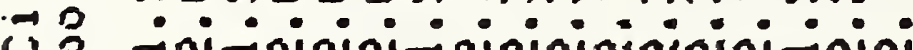

32

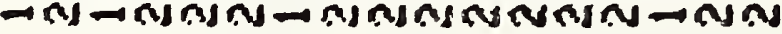

$=2$

I - TIMMMMT

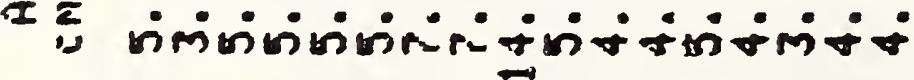

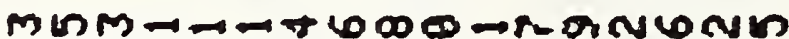

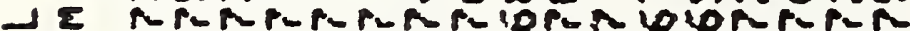

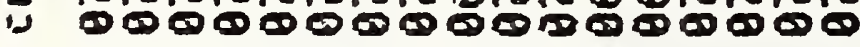

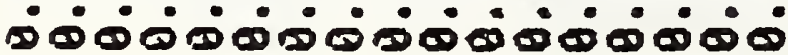

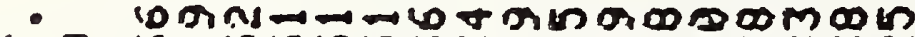

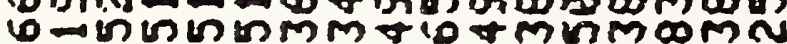

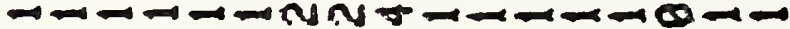

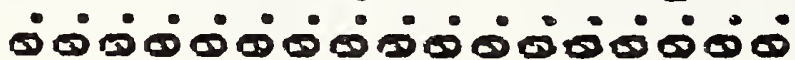

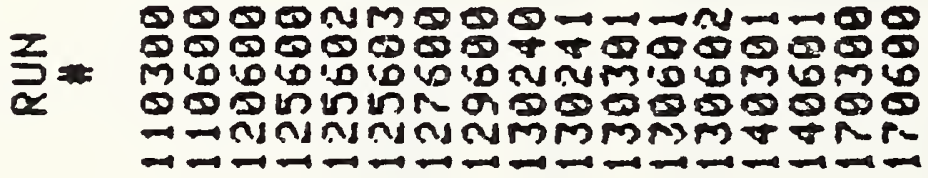

$+$

5

2

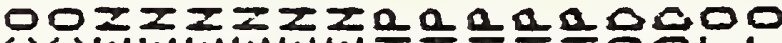

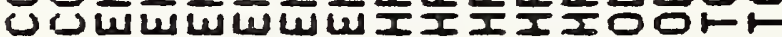
nen $\operatorname{con}$

.

$\infty+\infty$

$=$

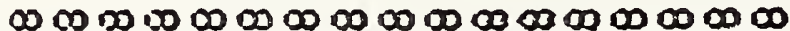

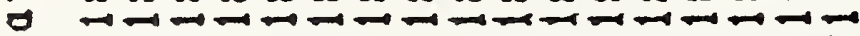

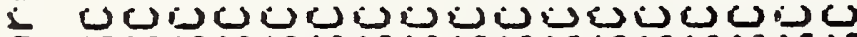

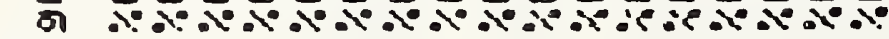

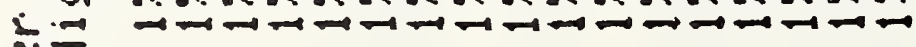

di $=$

5

2

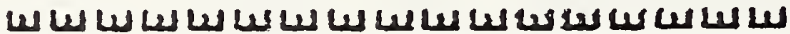

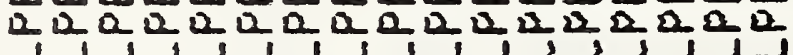




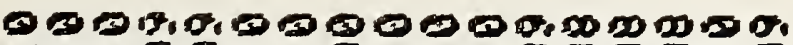

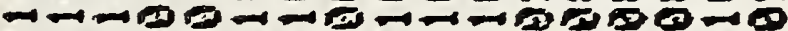

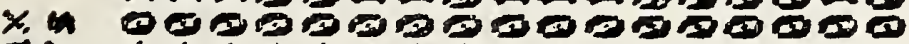

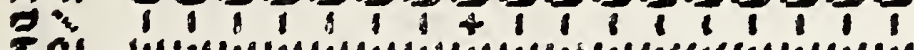

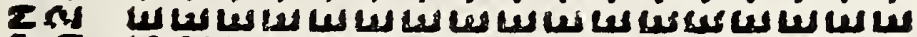

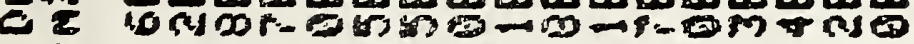

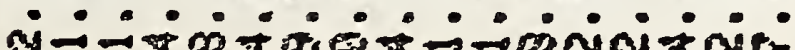

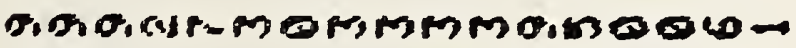
कن

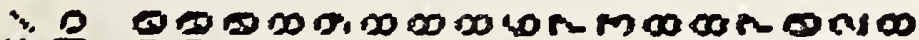

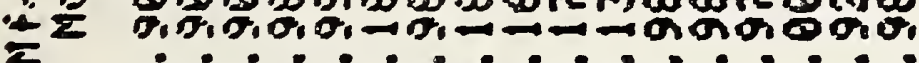

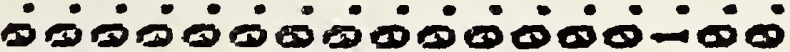
2 $\therefore \frac{1}{8}$ $\omega 2$ $\sum$ is $\checkmark m-\infty 00-\infty 00000 \times-m+\infty$

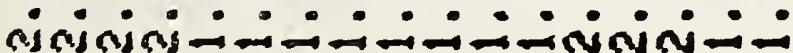

CI Hor

$2 \geq$

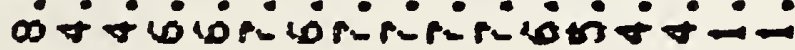

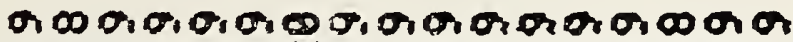

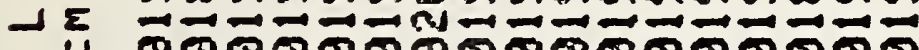

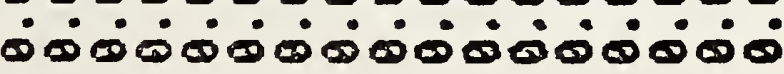

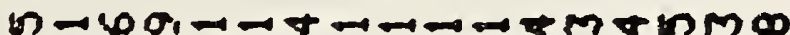

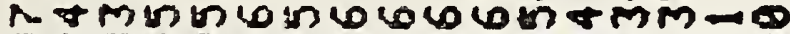

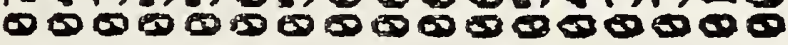
-

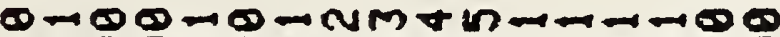
$\forall+000000000000000$

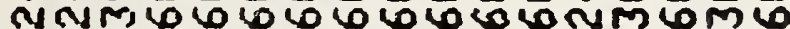
00000 in

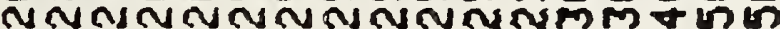

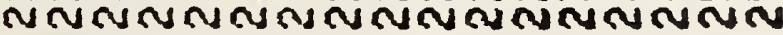

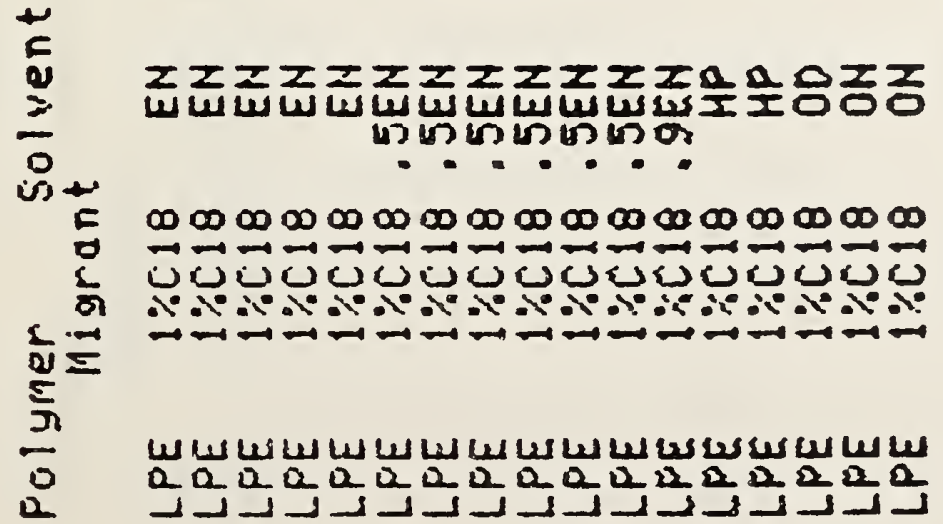




$$
\begin{aligned}
& \text { RDos op of } \\
& \text { os } 0
\end{aligned}
$$

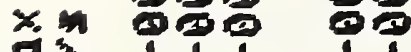

$$
\begin{aligned}
& 011111 \\
& \text { zar when wus } \\
& \text { Dom? } \\
& \rightarrow \text { mi }
\end{aligned}
$$

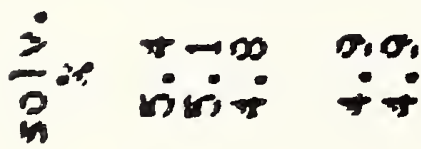

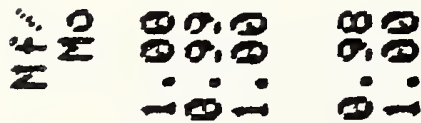

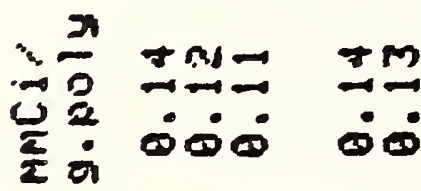

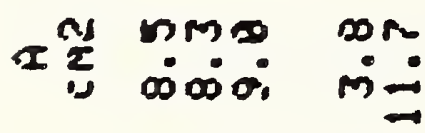

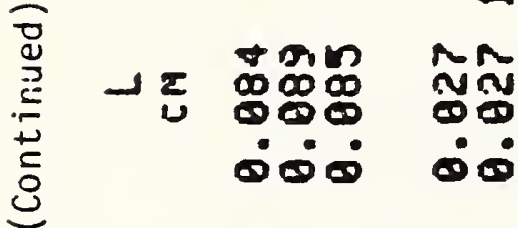

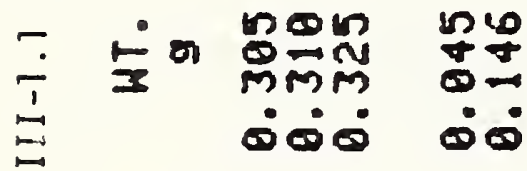

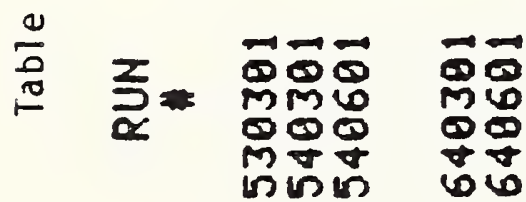

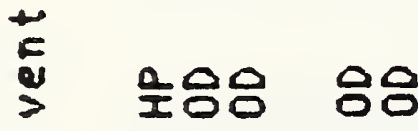

$$
\begin{aligned}
& \overline{0} \\
& =000000 \\
& \text { a } \\
& \text { 닌 }
\end{aligned}
$$

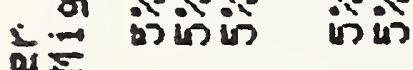

$$
\begin{aligned}
& \frac{5}{\pi} \\
& \frac{1}{3} \text { and }
\end{aligned}
$$




$$
\begin{aligned}
& 0 \\
& x \rightarrow 28 \\
& \text { zis ú } \\
& a \sum_{0}
\end{aligned}
$$

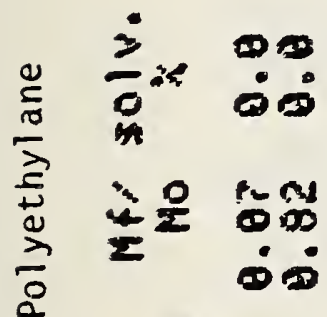

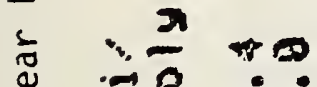

$$
\begin{aligned}
& \stackrel{0}{ \pm} \ddot{0} \\
& \text { I }
\end{aligned}
$$

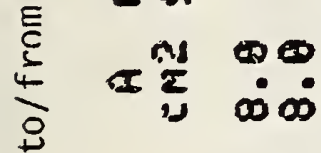

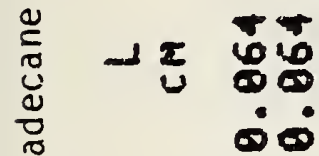

$$
\begin{aligned}
& \text { O } \quad M M \\
& \stackrel{1}{=}=M M \\
& \text { प } 50
\end{aligned}
$$

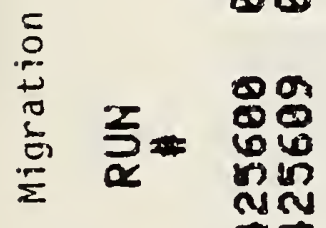

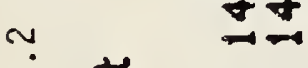

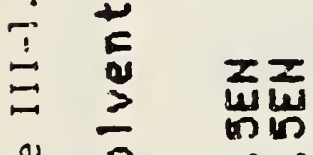

$$
\begin{aligned}
& \text { \& } 0 \text { แ } \\
& \text { 茂 } \\
& \text { 角 } \\
& \text { 둥 } \\
& \frac{\sum^{2}}{2} \text { us }
\end{aligned}
$$




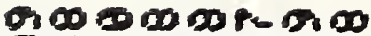

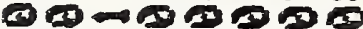

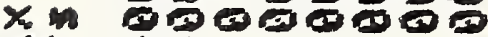

- 4. 1511111

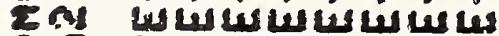

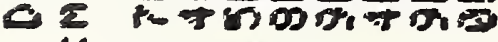
$-\circ \circ$. anisent $40 \div$

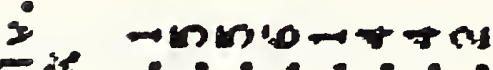
$\because-\infty \dot{0}-\dot{0}-\dot{0}$

is

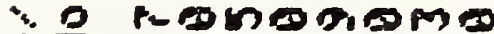

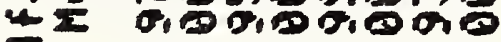
$\Sigma$ $\dot{0-\infty}-\dot{\infty}-\dot{0}$

$\pi$

$4 \div$

18

2:

$M-r-\infty, \infty 0 m n$

ns $x \rightarrow-1-m$ n

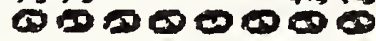

- 0000000

M M $T$ MASMM

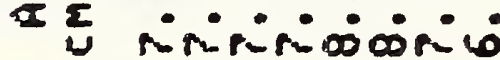

Tros PNOW

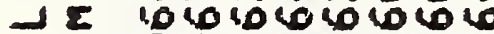

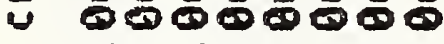
000000000

- Moroom- $\rightarrow$

- a orocor 0 or

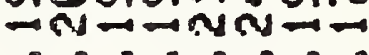
कம்

5
$\frac{5}{0}$
$\frac{0}{2}$
$\frac{0}{2}$
$\frac{1}{2}$
$\frac{1}{2}$
$\frac{0}{0}$

$\frac{2}{\alpha}=$ 00000 MLMLML

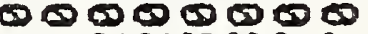
$\rightarrow-\infty n m m n$ NARLPLRMR

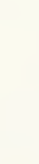

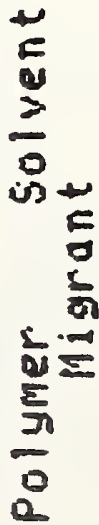
00 준둔

$\infty 0000000$ $\rightarrow-m-\infty \rightarrow-100$ UU⿺⿻一⿰冫⿰亅⿱丿丶丶⿱⿰㇒一乂 $x \cdot x \cdot x^{\circ} x^{\circ} \cdot x^{\circ}$

$\rightarrow-\infty-10$

แผแน

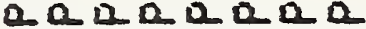
0000000000000

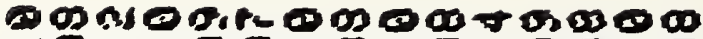

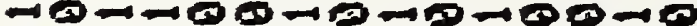
ore

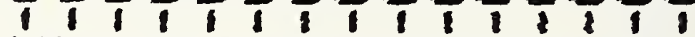

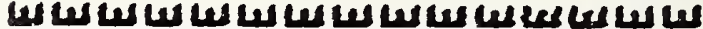

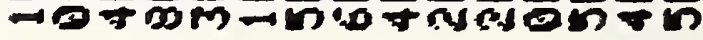

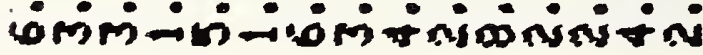

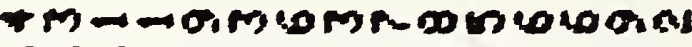

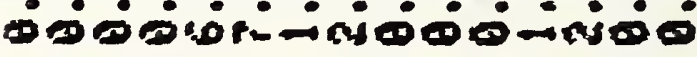

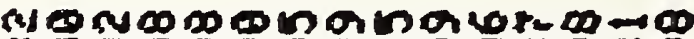

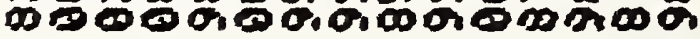

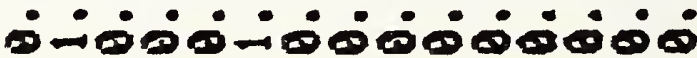

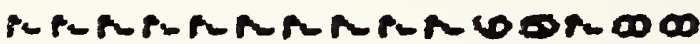

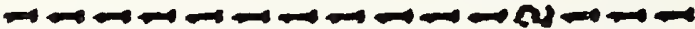

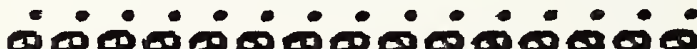

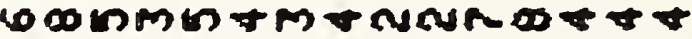

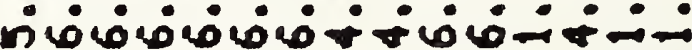

WR

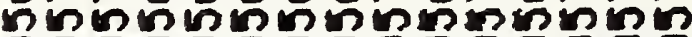
600000000000000

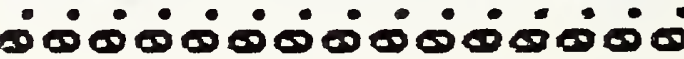

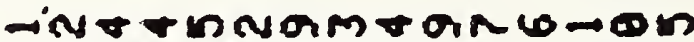

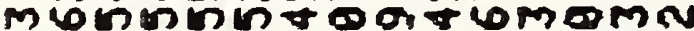
$\rightarrow-1-1-1-6-10-00$ is 0 is

$0000--0000000000$ 000000000000000 M

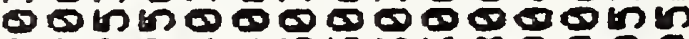
NNMN+

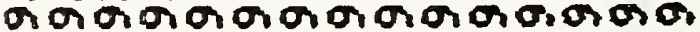

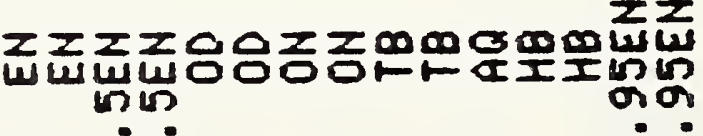

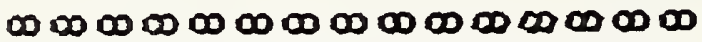

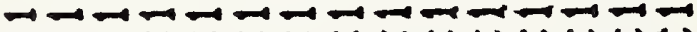

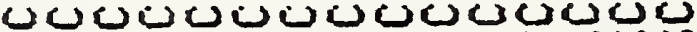

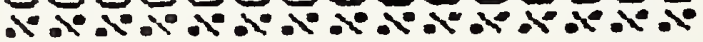

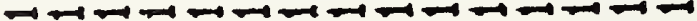

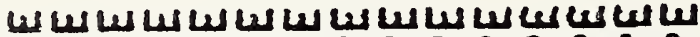

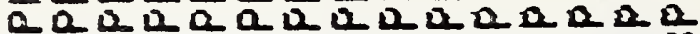

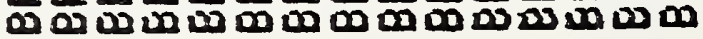




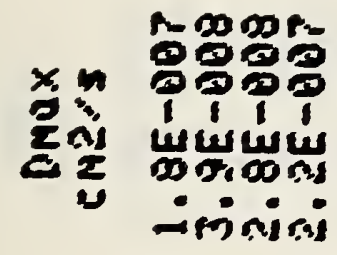

$\frac{2}{m}$ morises

4욘

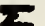

is

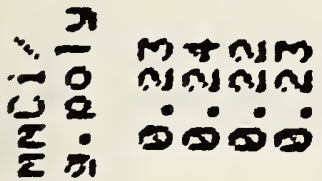

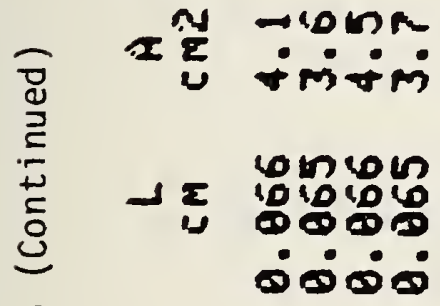

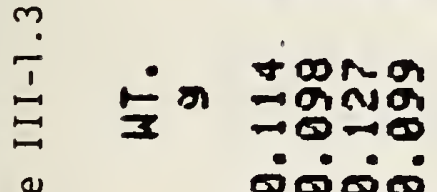

- $\quad 0000$

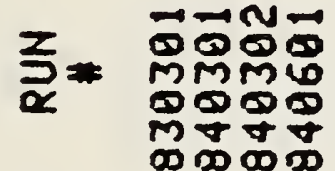

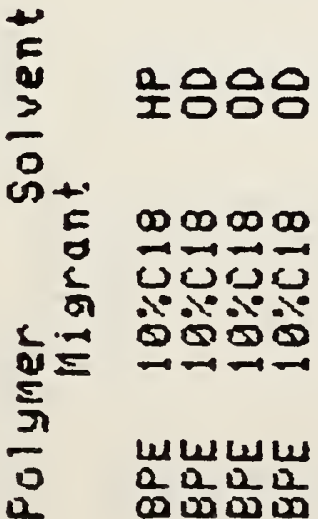




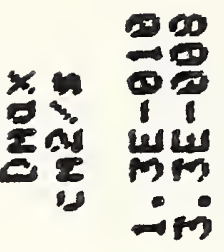

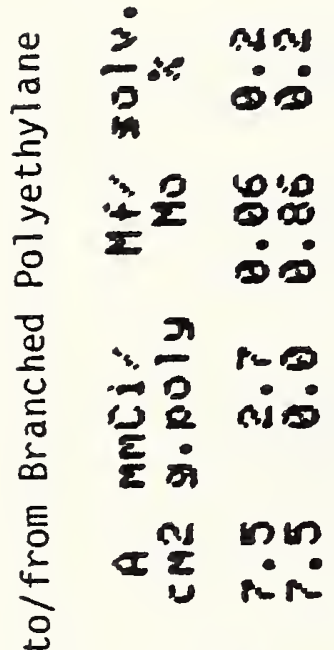

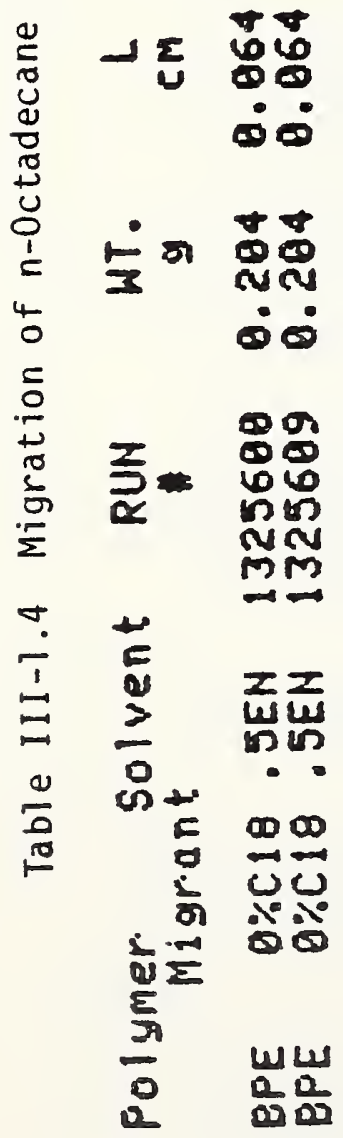




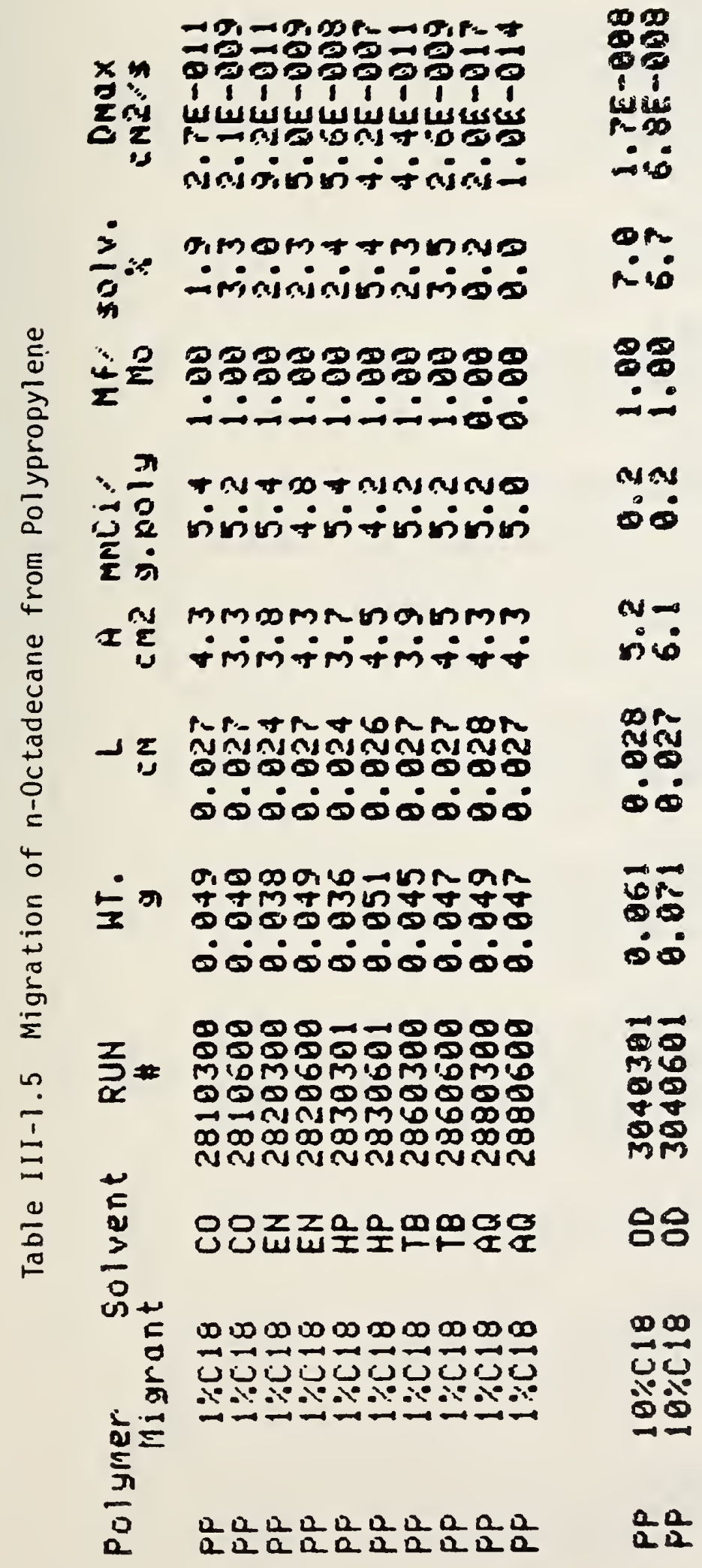




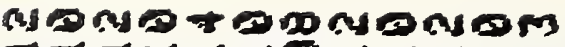

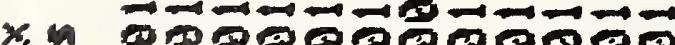

$2 \%$

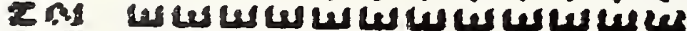

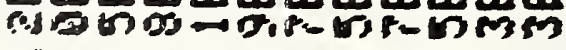

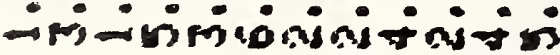

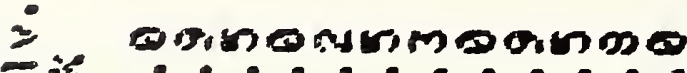

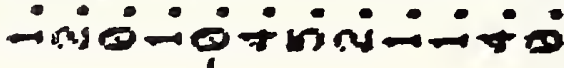

if 1$$
1
$$

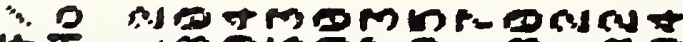

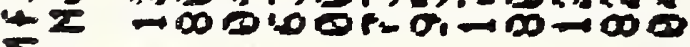

$\sum$

is

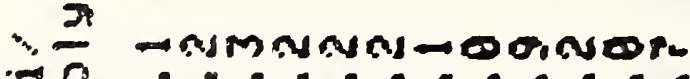

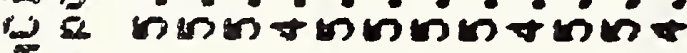

2

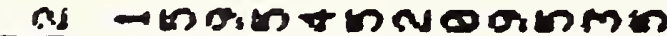

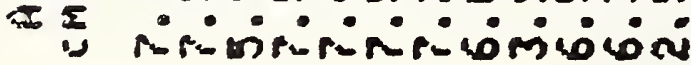

$0,0,000, \infty$ on 00 on

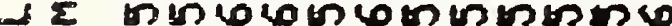

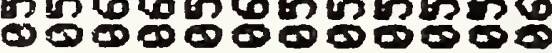

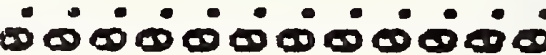

thmmogo-10-10mo

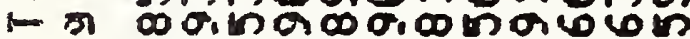

I $\quad-1 \rightarrow-4-\infty-0=0$ $\therefore-10000000$

600060-1000000

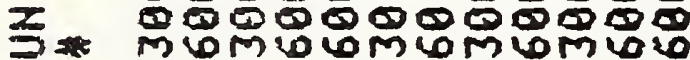
0010 - WNRMmintran or or oiororoiono

$+\quad-\infty+\infty-\infty-\infty-\infty$

E

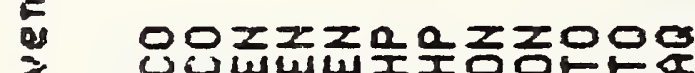

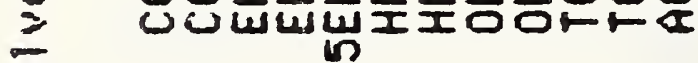

o ?

CH

- mancinarumanes

- MMMMMMNMMMMM

क ن

20

$\underline{5} \pm$

5

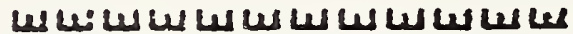

- Laaaaaa․

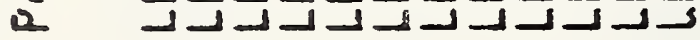




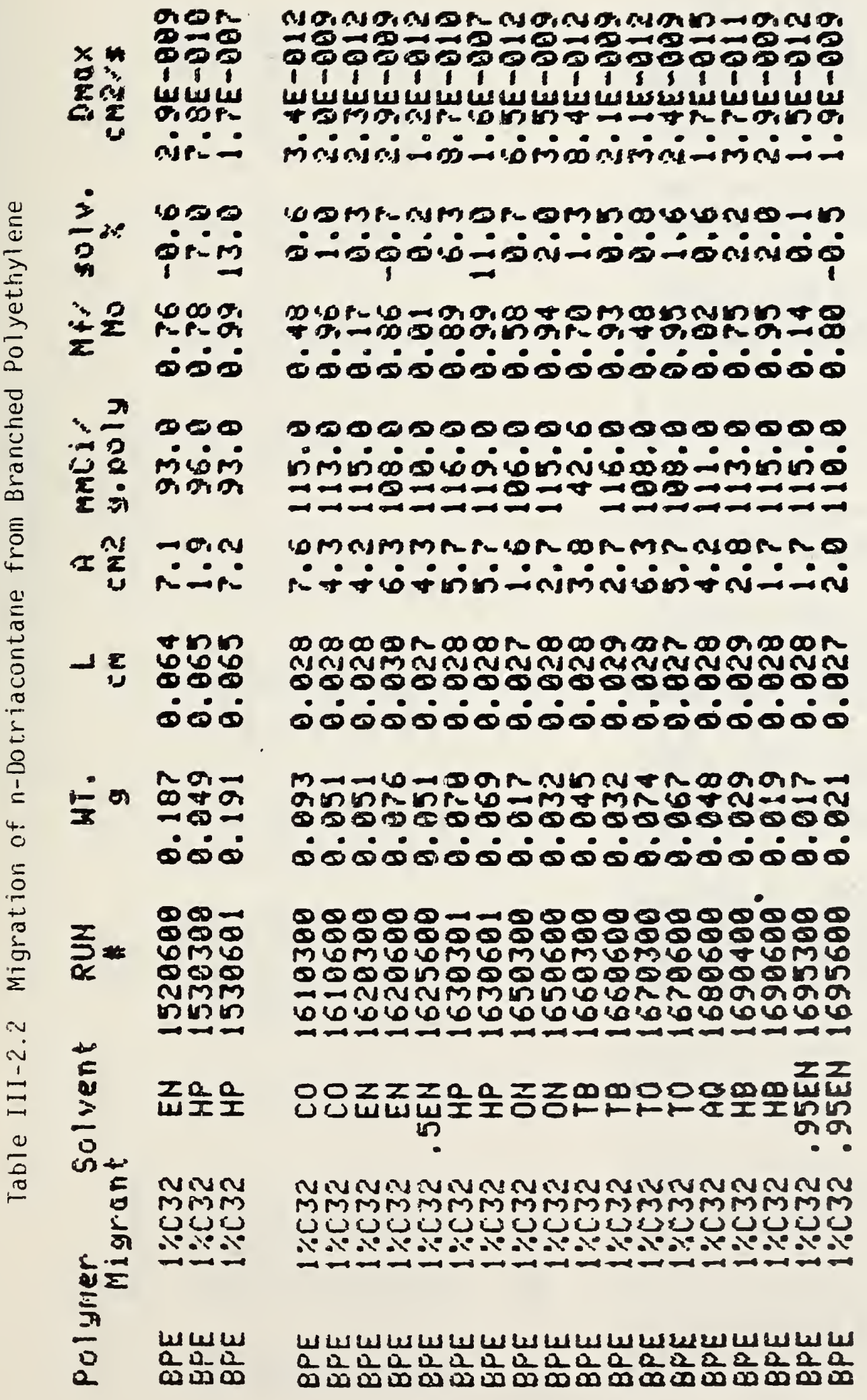




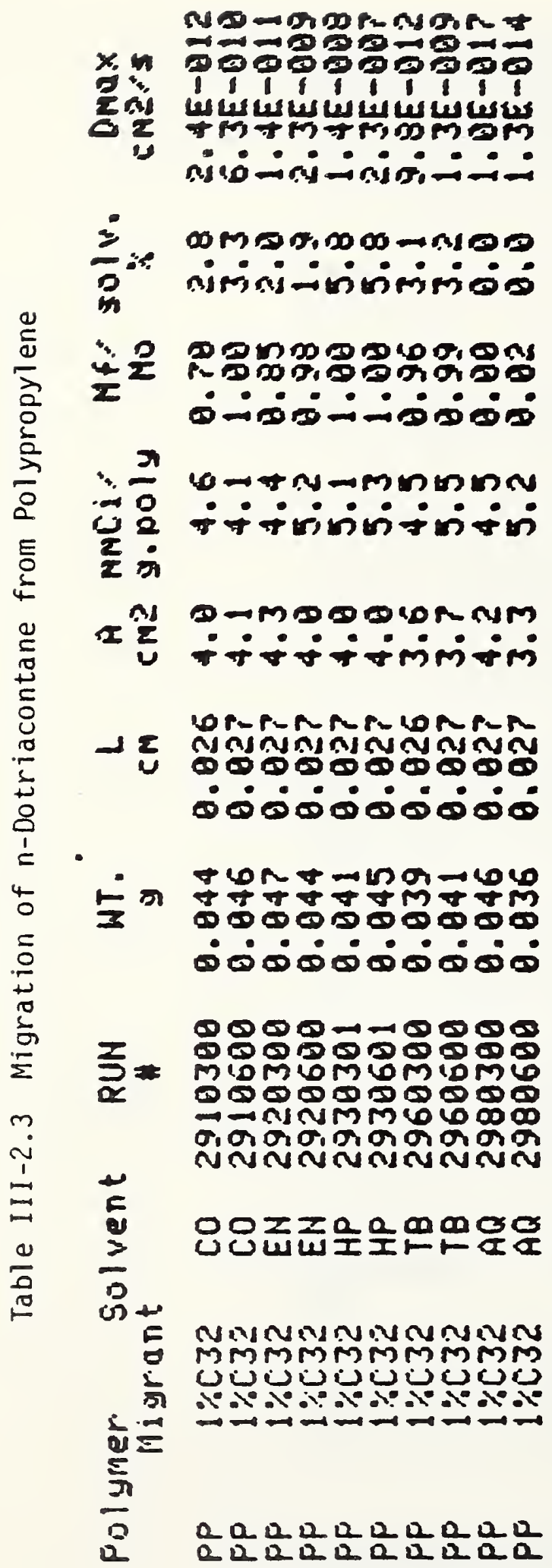




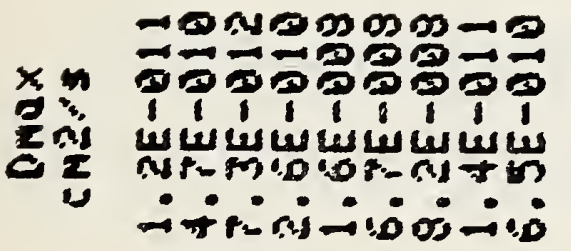

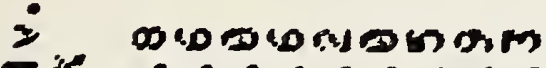

$\frac{2}{n}$

oni-i

ㅇ

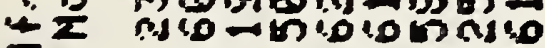
$z$

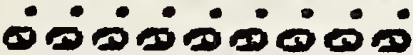
$\pi$ $\therefore \frac{1}{20}$ 20 ह

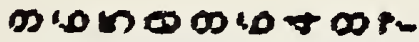

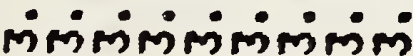

on montom

$2 \sum_{1}^{2}$ Disiminisis

$00 \rightarrow-0 \rightarrow-96$

$d=10.060400000$

is வ்ம்க்க் ம்

- Gingarntab -m ninnosomis $\rightarrow-1-0 \rightarrow-1+10$ ம் ம் ம்

0000 $\sum_{0}=$ 000000000 MLMGMWOMN 000000000 - $=$ NIMMMNR 000000000 लกNMNMNN

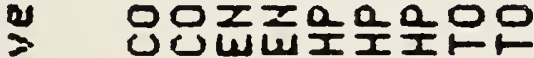

$\frac{1}{0}$

ज十

E トレトトトトトトト

조ㅍㅗㅗㅗㅗำ

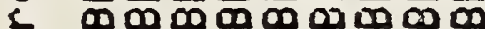

on

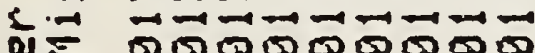

y $=0000000000$

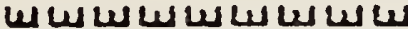
a c i a a a a n

$\infty$

1

4

$\because$

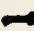

as

$\div$

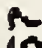

(2)

4

$\infty$

$\dot{*}$

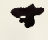

i

$\infty$

N

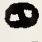

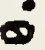

$\infty$

s

$\infty$

0

$\overrightarrow{0}$

0

$\infty$

m

$\vec{N}$

모

$\frac{1}{2}$

$\frac{T}{0}$

$\therefore$

$\infty$

.

u

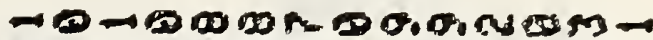

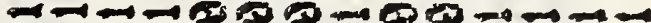

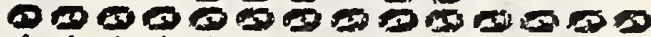
$111111.111 \% 1$

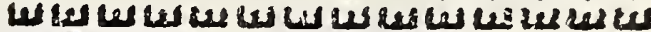
- 0 \%

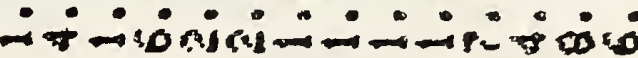

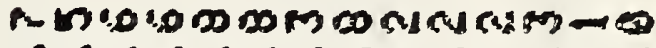

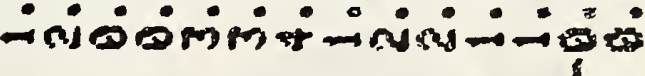

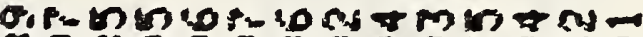

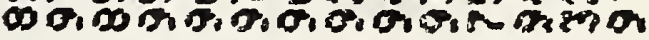
is

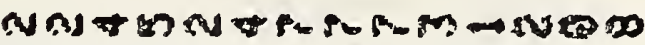

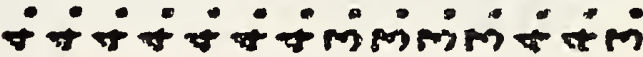

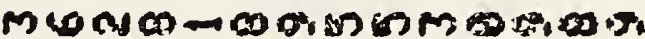
ம்

ORPHAR 606060600

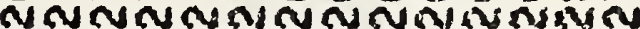
0000000000000

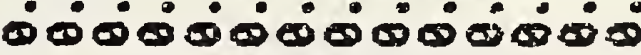

NMMAMNR- REWHEON

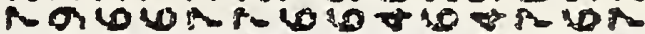
00000000450000

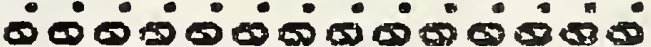

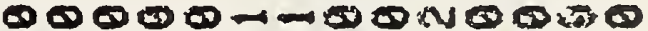
00000000000000 MLMLMMLMLLMLML 00000000000000 - - N NMMMnMU

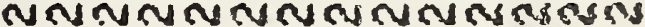

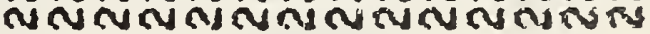

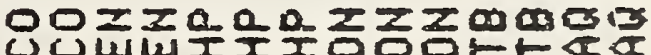

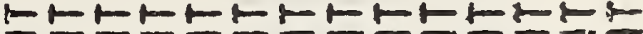

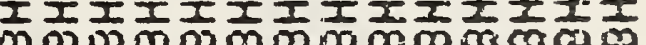

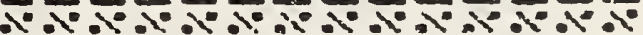
1 00000000000000

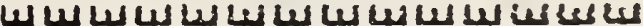

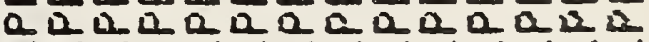

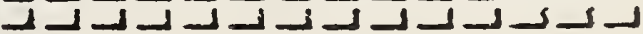




$$
\begin{aligned}
& \text { ocos } \\
& -100 \\
& x, 0000 \\
& \text { 2 i } 11 \\
& \text { zos unus } \\
& \text { is } 20= \\
& \text { nopi- } \\
& \text { in misió }
\end{aligned}
$$

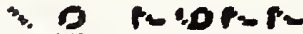

$$
\begin{aligned}
& \angle 20.00 \% \\
& \Sigma
\end{aligned}
$$

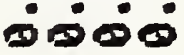

$$
\begin{aligned}
& \therefore \frac{\pi}{0} \text { n.upto } \\
& \text { ua ninnu } \\
& \text { ㅎํ }
\end{aligned}
$$

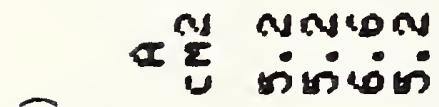

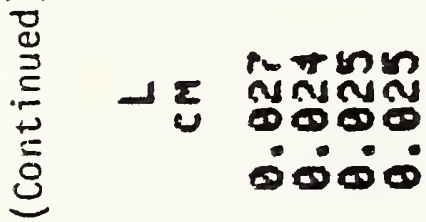

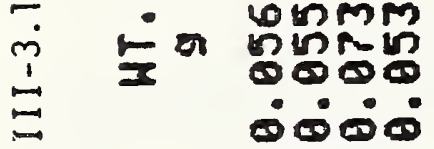

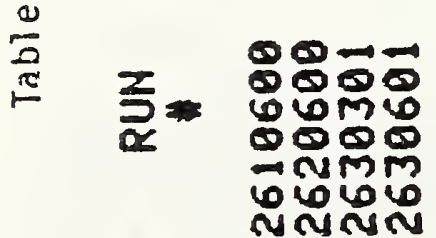

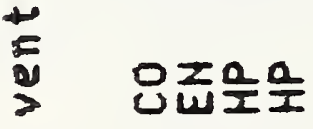

$$
\begin{aligned}
& 0
\end{aligned}
$$

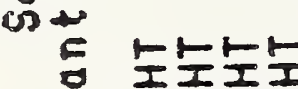

$$
\begin{aligned}
& \text { - III퐁 }
\end{aligned}
$$

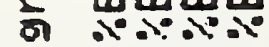

$$
\begin{aligned}
& \text { iो } \\
& \frac{2}{\pi}=000 \\
& \text { - uس山س } \\
& \text { a ana }
\end{aligned}
$$




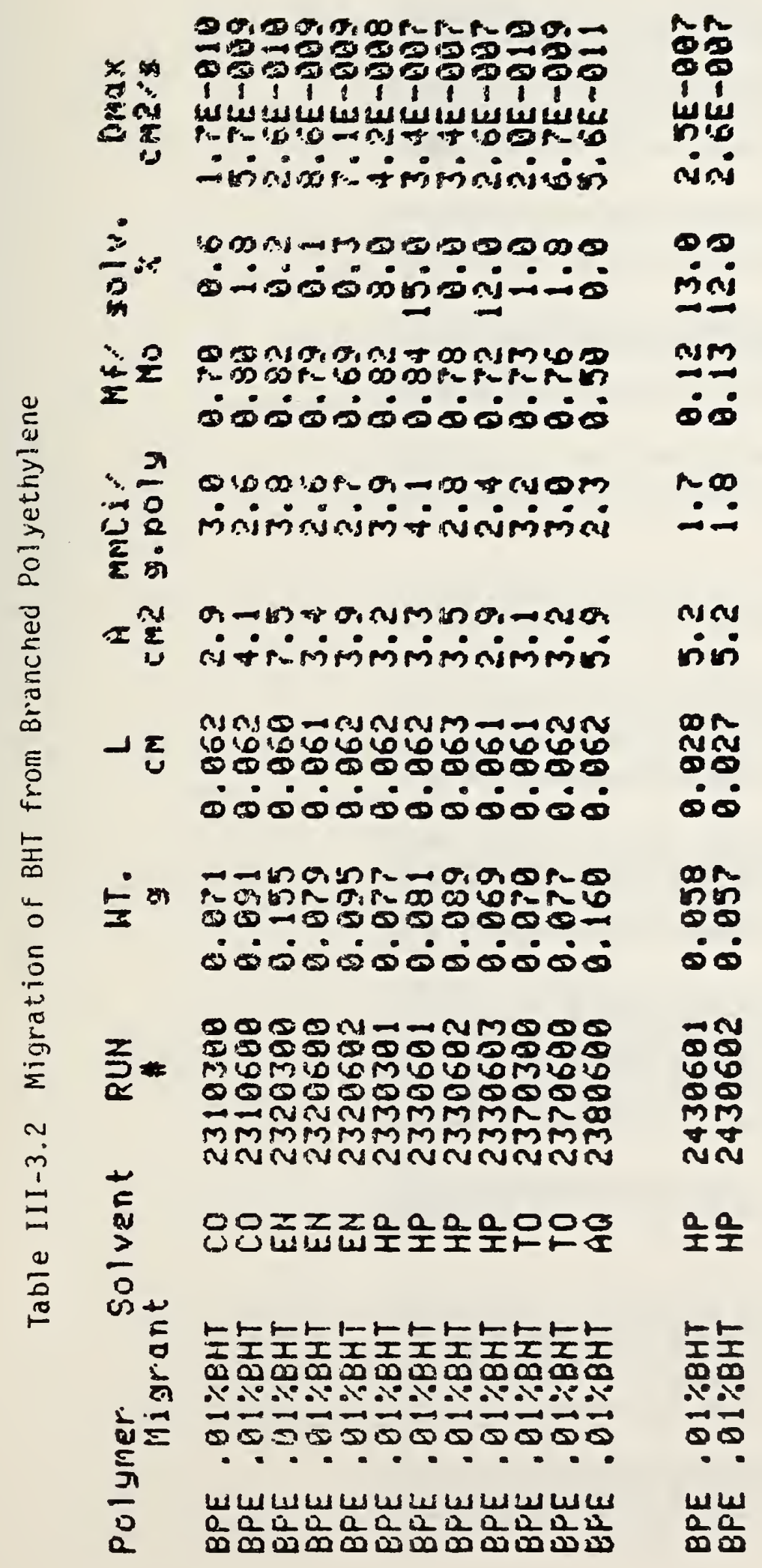




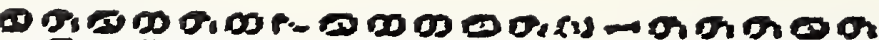

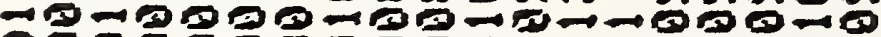

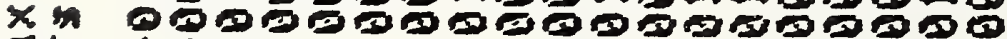

2\% $11,1,1,1,1,11,1$

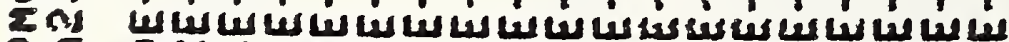

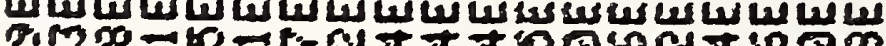

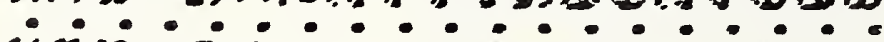

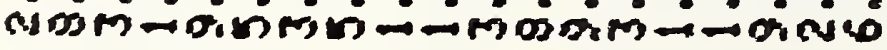

$\frac{2}{2}$

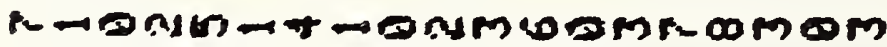

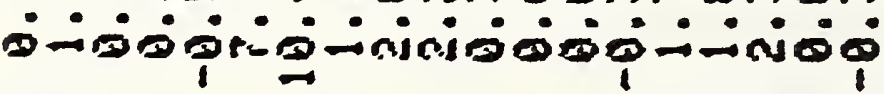

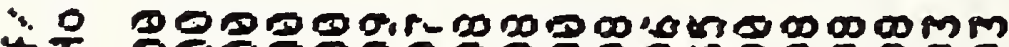

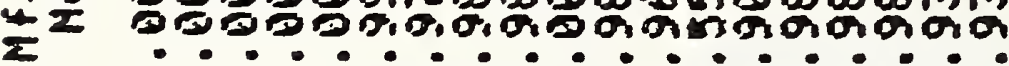

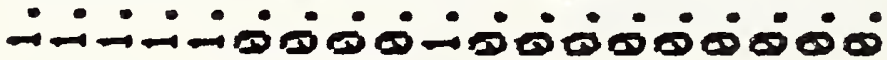

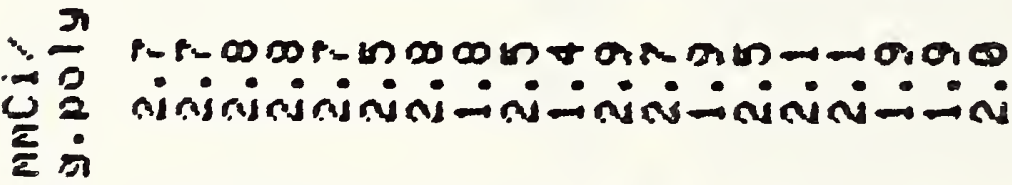

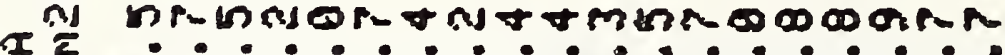

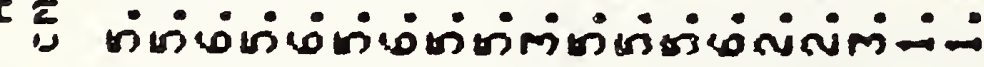

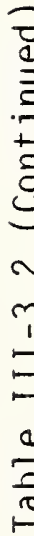

PLPR-

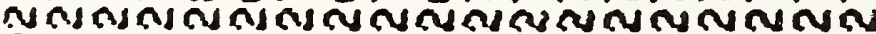
0000000000000000000 ن்

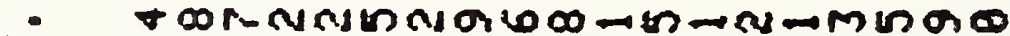

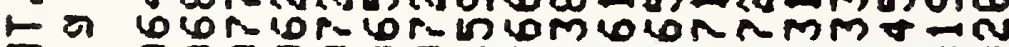
I 00000000000000000000

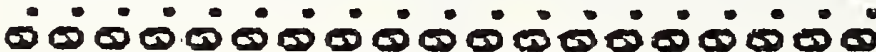

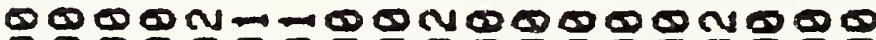

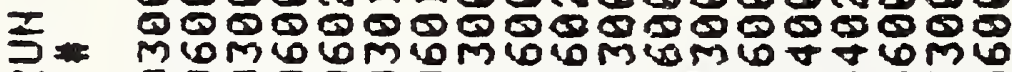
00000000000000000 - añmmin v

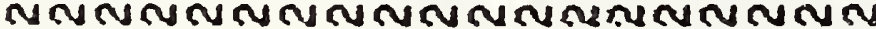

$+$ 즌 $00 z$ 주은

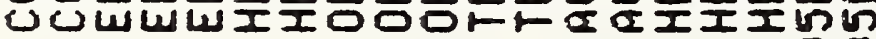

(2)

ن) + ?

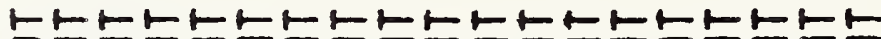
IIIIIIIIIIIIIIIIIIII

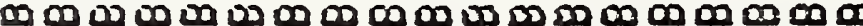

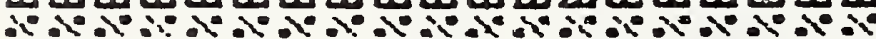

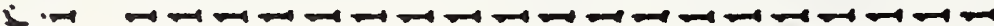

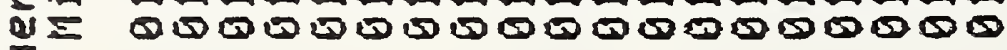

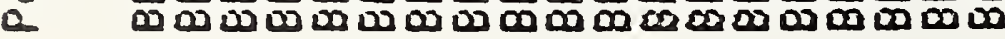


momost-nom-

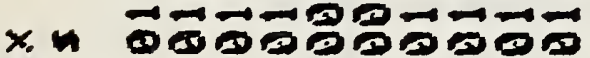

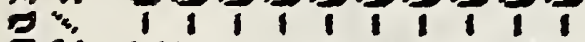

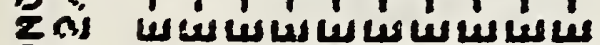

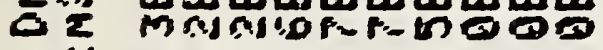

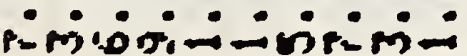

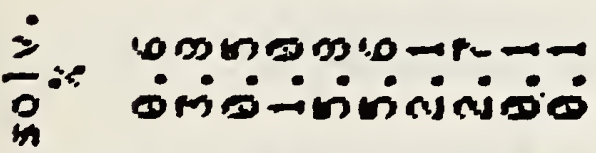

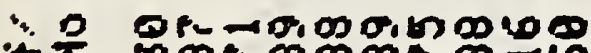

$4 \frac{2}{2}$ MmPDs

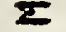

-

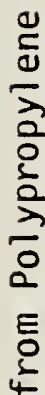

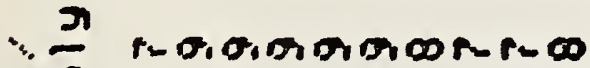

$\rightarrow 0 . \cdots . \cdot 1 . .$.

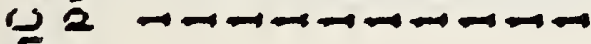

要

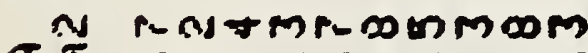

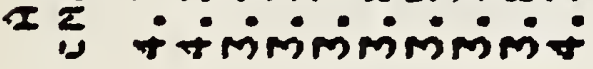

MANLDDORMR

- I MNMNNMNMNA

0000000000

نं

4

동

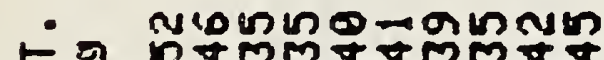

0000000000

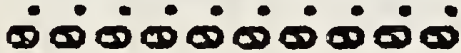

$\sum$

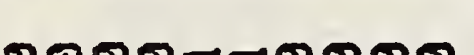

m $\quad 0000700000$

Z

MLMOMOMOM⿻

0000000000

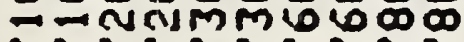

rnRrnRnRn

F

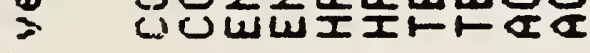

D

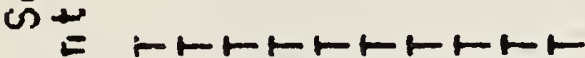

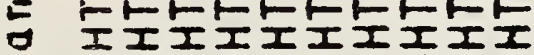

- 000000000000

争

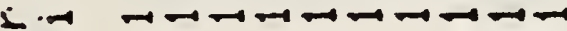

ए $=000000000$

ก)

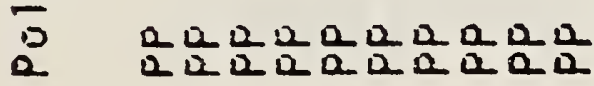




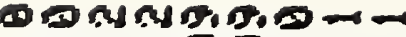
$\Rightarrow=-00-1=$

$x$ 400000000

2" 11191111

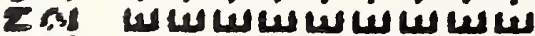

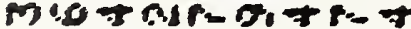

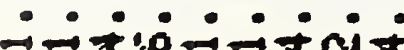

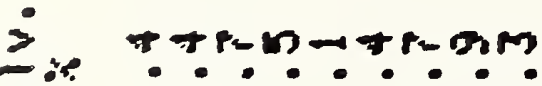

2

$\rightarrow$ ris $\rightarrow$ sisins

$\because \frac{9}{2}$

a

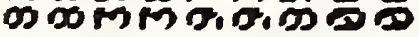
in் $\pi$

$\div \frac{1}{2}$

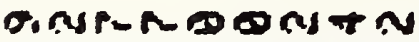

12

-ni-1000in

$\sum \dot{2}$

ns -DAMMMNMn

$2=$

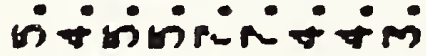

malmodormer

$+\frac{z}{v}$

niclopweon

000000000

ن்

- $\quad 1000001-70$ ton

-n

건 1000

ڤ

$0000-\infty 000$

000000000

MMMMMMMMM

000000000

$\Rightarrow r=n=R-\infty=6$

$\rightarrow$ mmra arom

$\omega$

8

-

$\infty$

$\infty$

$m$

$\infty$

$\infty$

$\frac{z}{a}=$

$\dot{8}$

$\Xi$

$\frac{1}{0}$

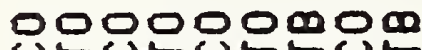

UTURUFHUF

$\stackrel{\infty}{1}$

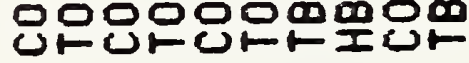

जम

$=\infty 00000000000000$

व $\rightarrow \overrightarrow{7} \overrightarrow{-1} \overrightarrow{\mathrm{O}} \overrightarrow{\mathrm{B}}$

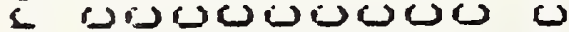

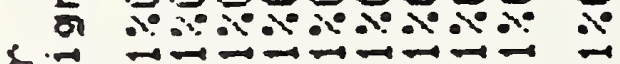

$\infty 0000000000$ $\rightarrow-1 \rightarrow-\infty \rightarrow-\infty$

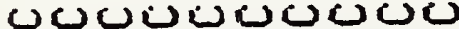

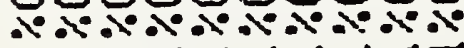

$\sum=\infty 0$

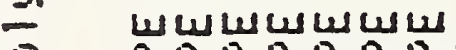

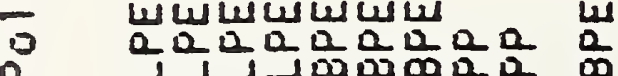

$-\rightarrow-1-1-10-1$ $\infty \infty$

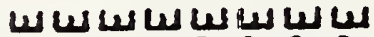

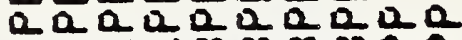
$1]+1000000$ 


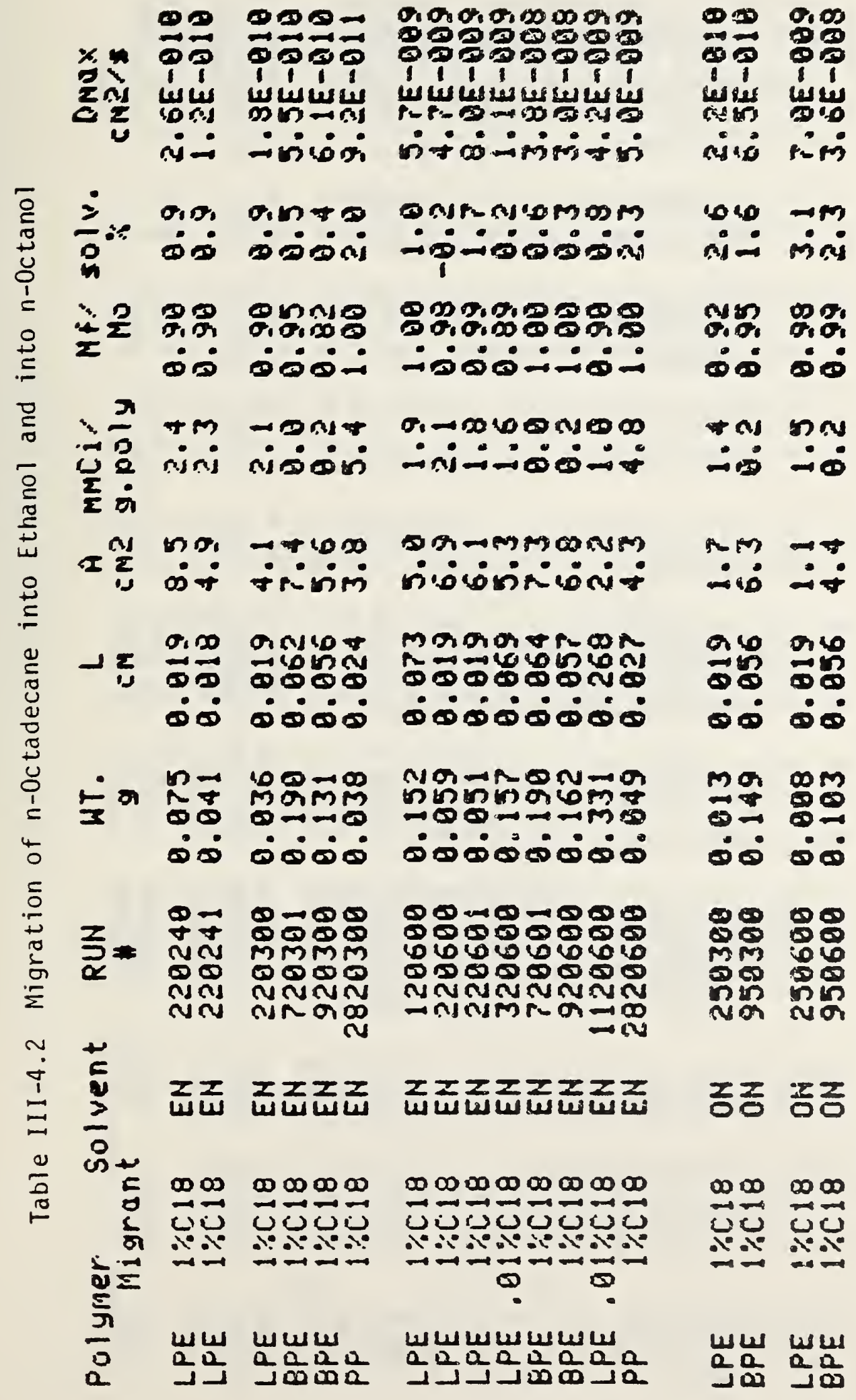




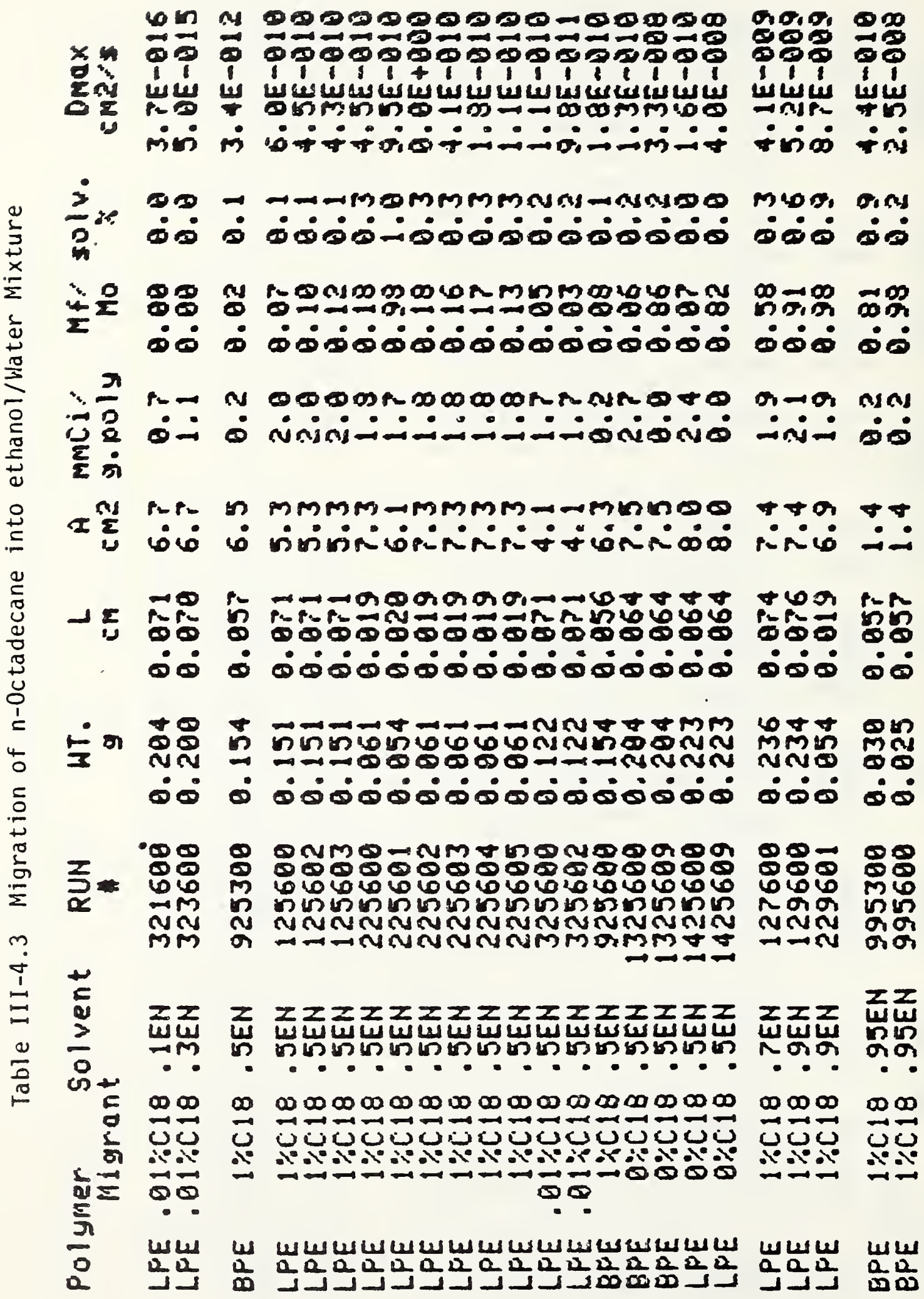




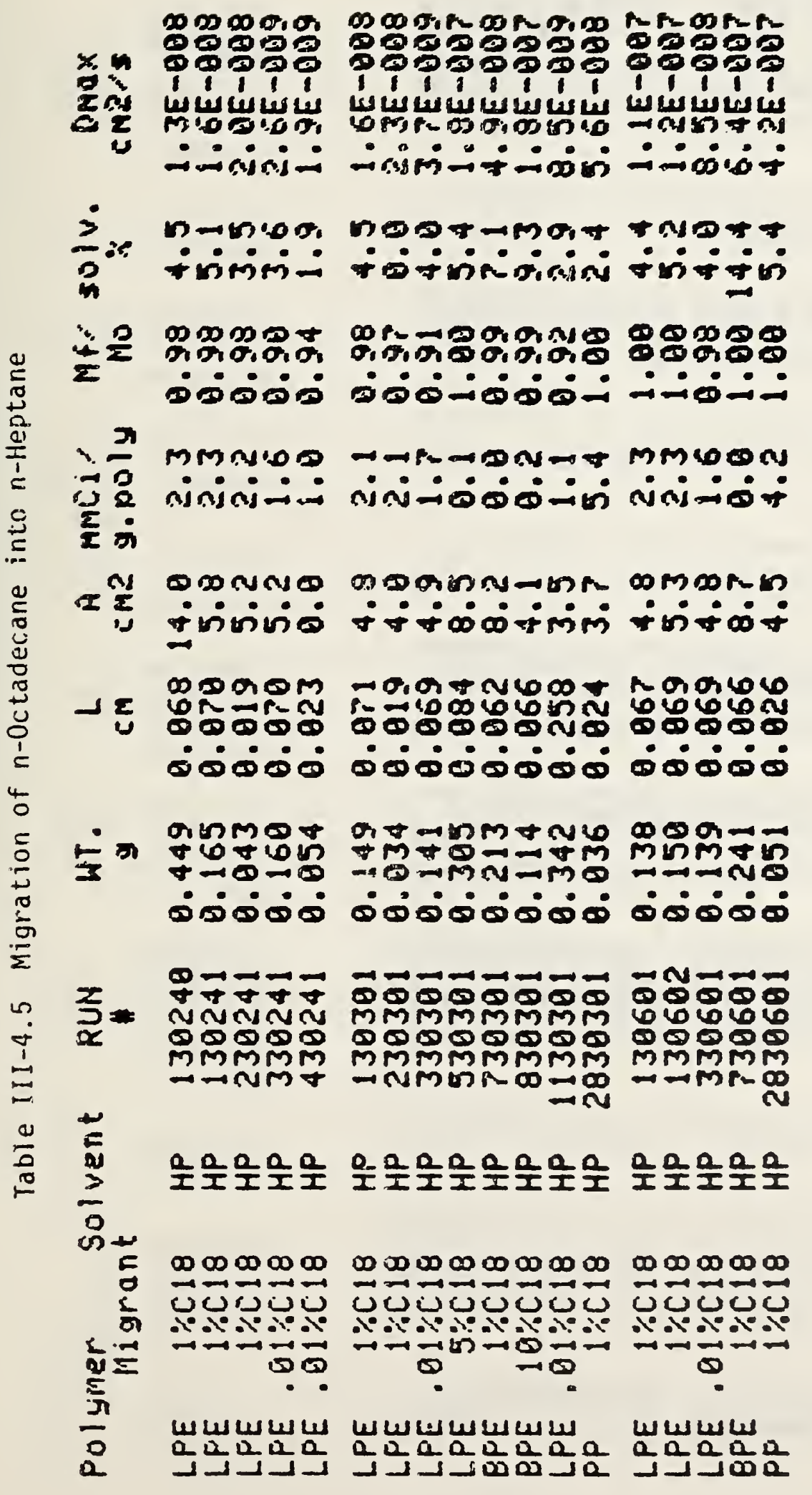




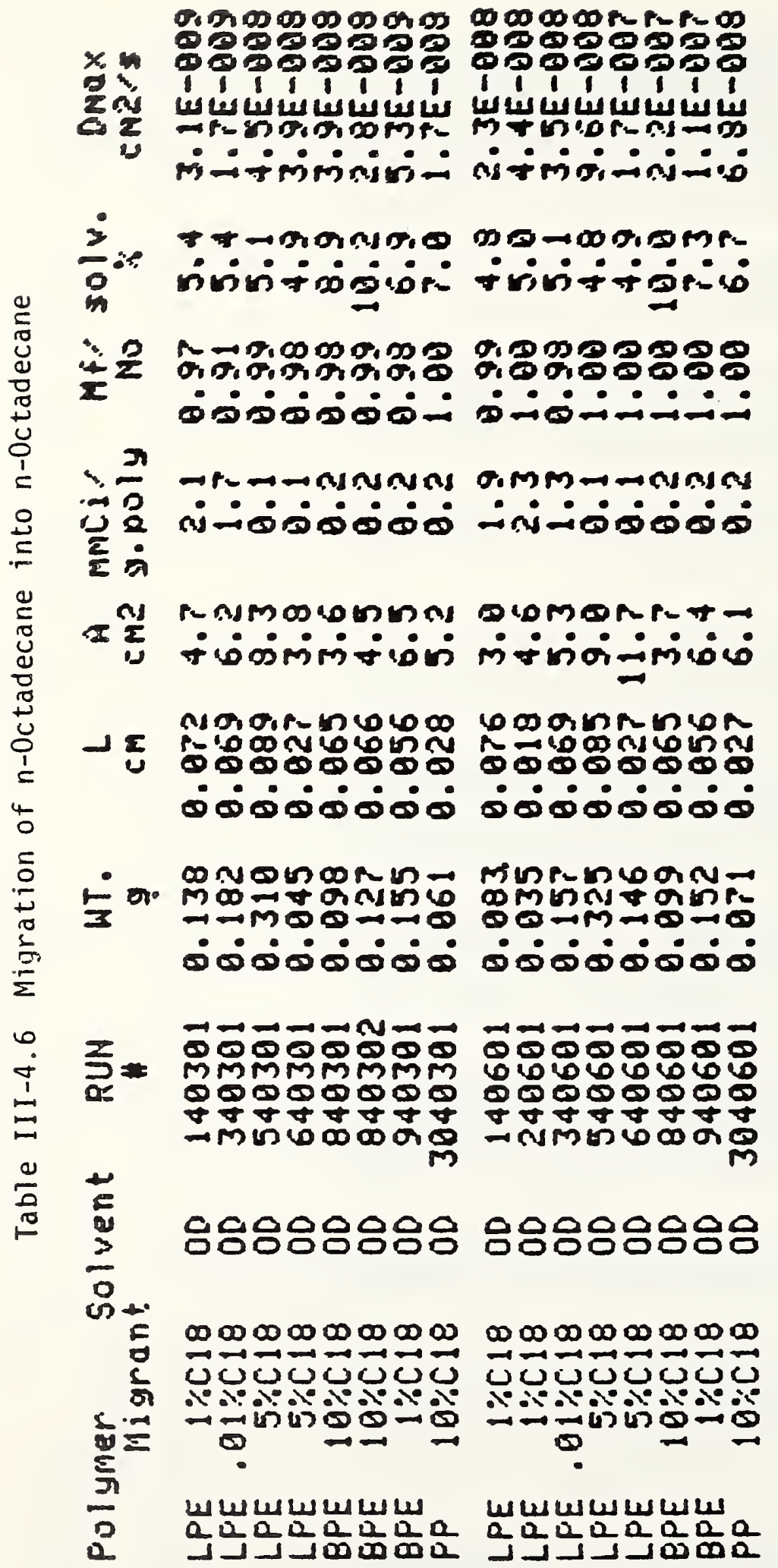




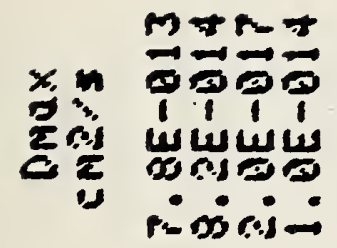

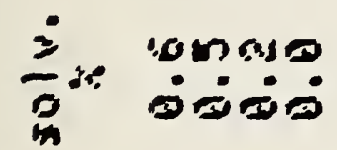

$$
\begin{aligned}
& \div \frac{0}{2}=000 \\
& \stackrel{1}{3} \quad \frac{\pi}{\pi} \operatorname{lng} 0
\end{aligned}
$$

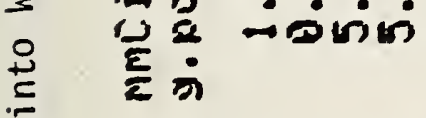

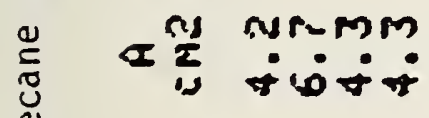

$$
\begin{aligned}
& \text { 苛 } \quad \text { or } \\
& \text { U }-\frac{E}{0} \text {, } \\
& \text {. } 000000 \\
& 4
\end{aligned}
$$

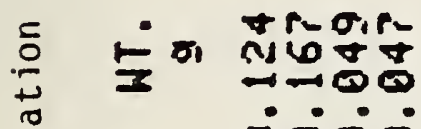

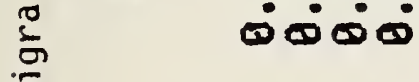

$$
\begin{aligned}
& \text { 文 } 0000 \\
& -\quad \sum=0000 \\
& \text {; } \overline{0} 00000 \\
& \text { Đ } \quad \text { Mo. }
\end{aligned}
$$

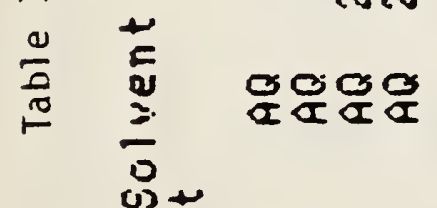

$$
\begin{aligned}
& \text { E } 000000 \\
& \text { 요 } \rightarrow-1 \\
& \text { 再 }
\end{aligned}
$$

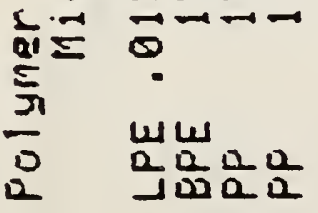




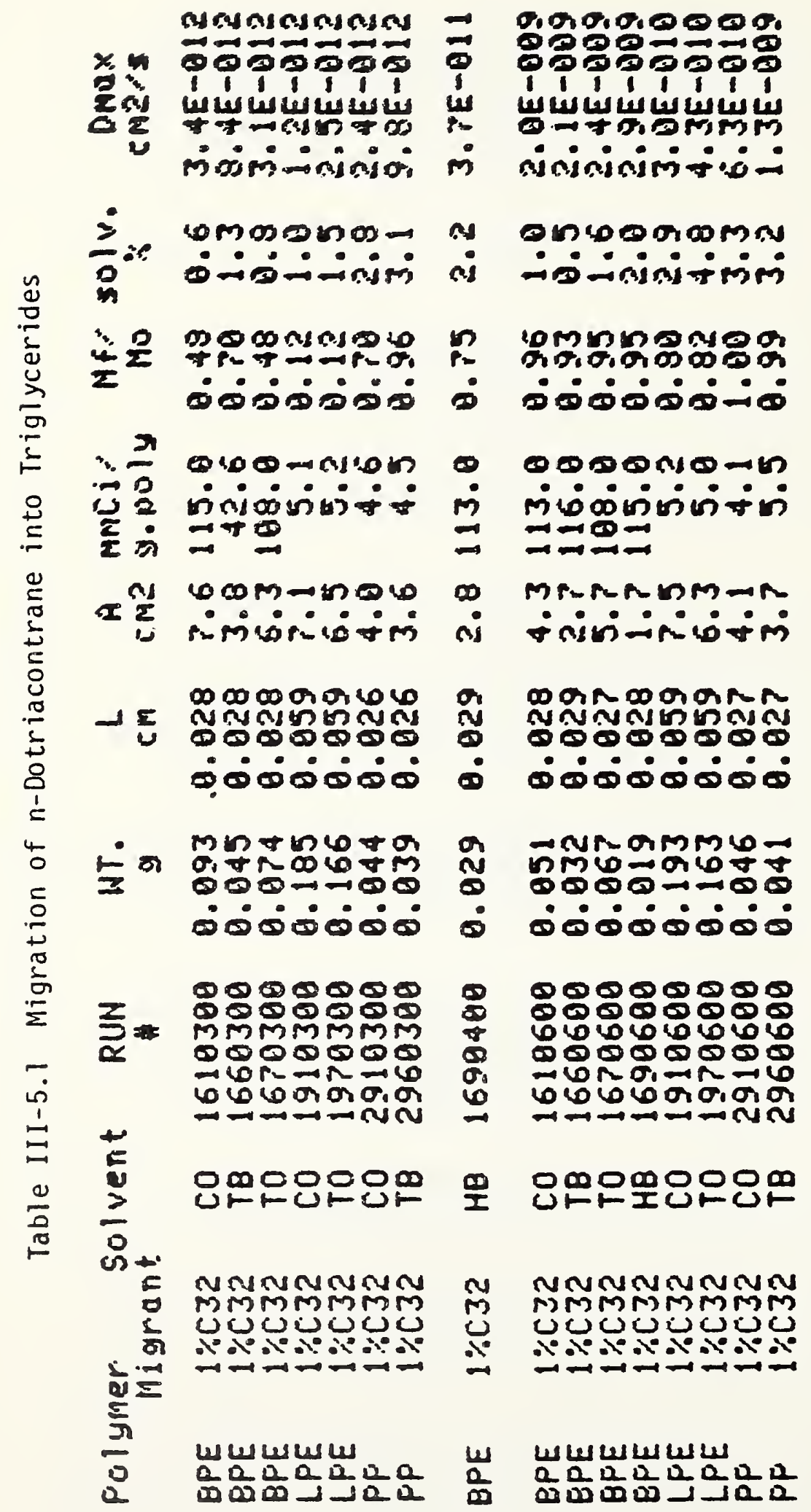




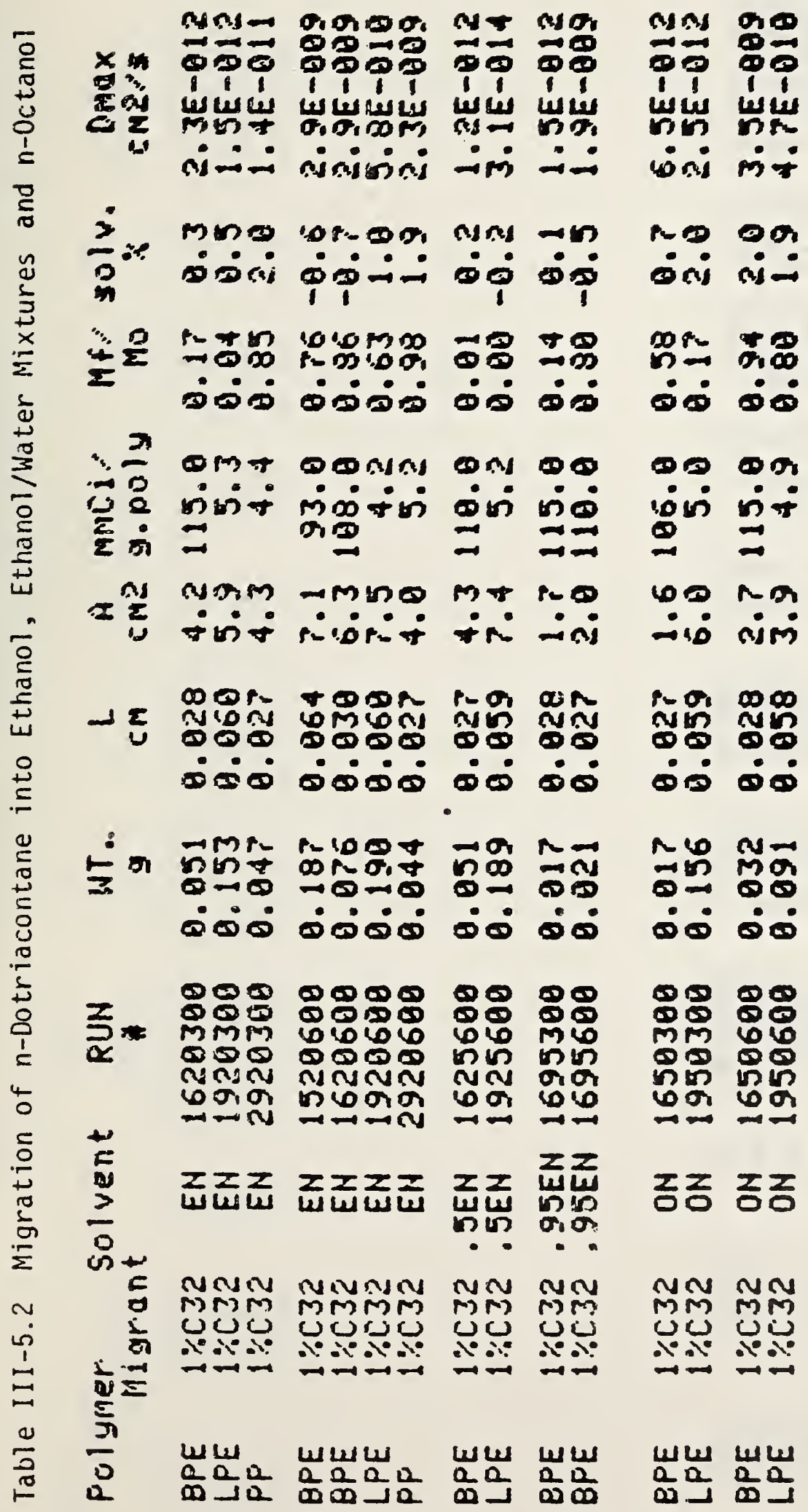




$$
\begin{aligned}
& \text { agom hropr } \\
& \text {-700 9000 } \\
& x * 0000000 \\
& \text { क\% } 111,111 \\
& \text { isuch whis } \\
& \text { कof } \\
& \text { Pisio-i inini }
\end{aligned}
$$

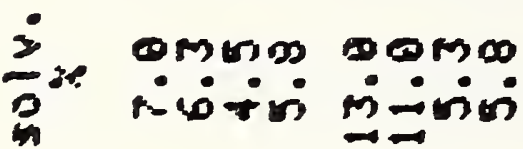

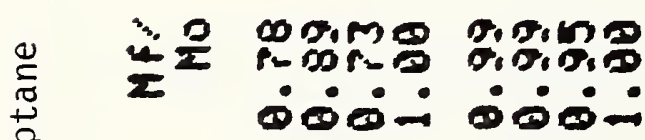

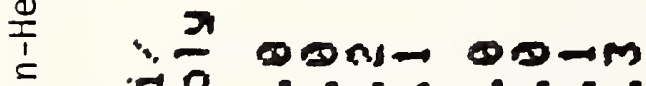

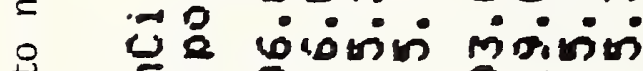

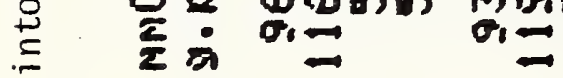

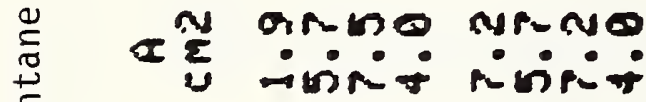

$$
\begin{aligned}
& \text { O } \\
& \therefore \quad=\quad 20-70000 \\
& \text { d } \\
& 00000000 \\
& \text { ம்க் }
\end{aligned}
$$




$$
\begin{aligned}
& \text { nMr- } \\
& \text { x क्षित्व } \\
& \text { है } 1 \\
& \text { zas whtulu }
\end{aligned}
$$

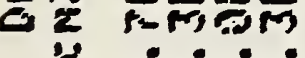

$$
\begin{aligned}
& \therefore \text { is } \\
& \frac{2}{9} \text { : }
\end{aligned}
$$

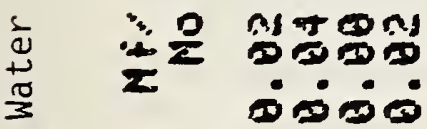

$$
\begin{aligned}
& \stackrel{0}{\therefore} \quad \frac{21}{0} \text { Drtins }
\end{aligned}
$$

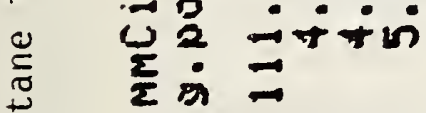

$$
\begin{aligned}
& \text { 요 nisnm } \\
& \text { i } \\
& \text { 范 } \\
& \begin{array}{l}
1 \\
4
\end{array}
\end{aligned}
$$

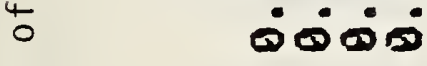

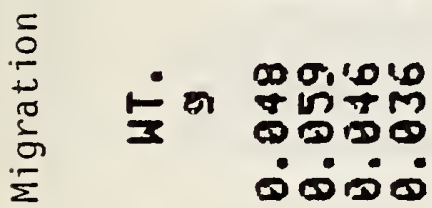

$$
\begin{aligned}
& \text { ㅁ }
\end{aligned}
$$

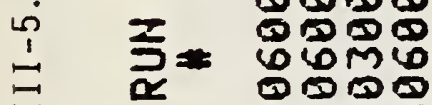

$$
\begin{aligned}
& \Rightarrow \quad \text { 口 } \\
& \text { ๑ }
\end{aligned}
$$

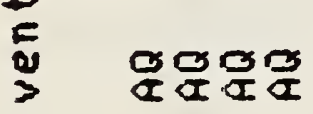

$$
\begin{aligned}
& \text { कि } \\
& \text { E mMmm } \\
& \text { क를 } \\
& \frac{5}{\pi}
\end{aligned}
$$

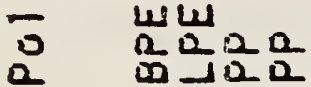




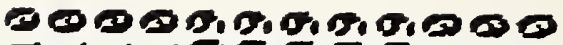

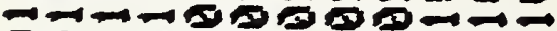
Q w

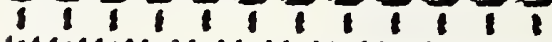

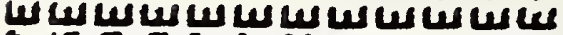

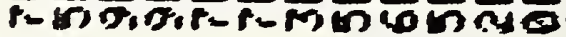
$\therefore 0$ :0

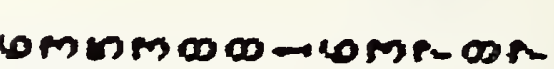
rivini-i-icimimi

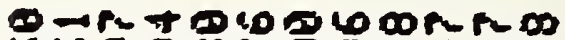
wios. -

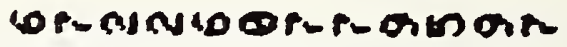

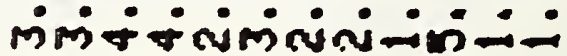

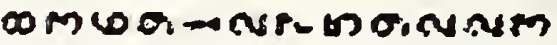

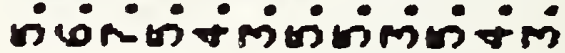

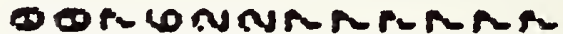
\% 000000000000

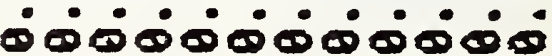

ns 6 M nionnon $-10000000000$

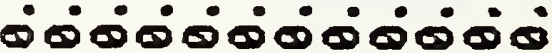

000000000000 006006000000 W 000000000000 $-n=w-n-\infty 0$

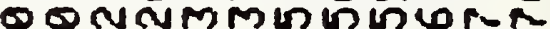
NलMNMRIN NUNMN

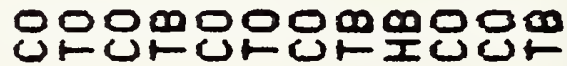

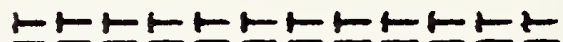

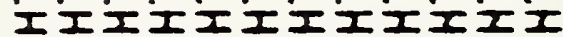
in

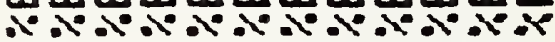
$\rightarrow-\rightarrow-\rightarrow-1 \rightarrow-100$ 000000000000

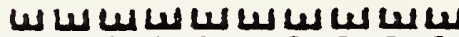

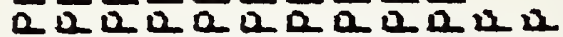

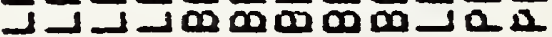




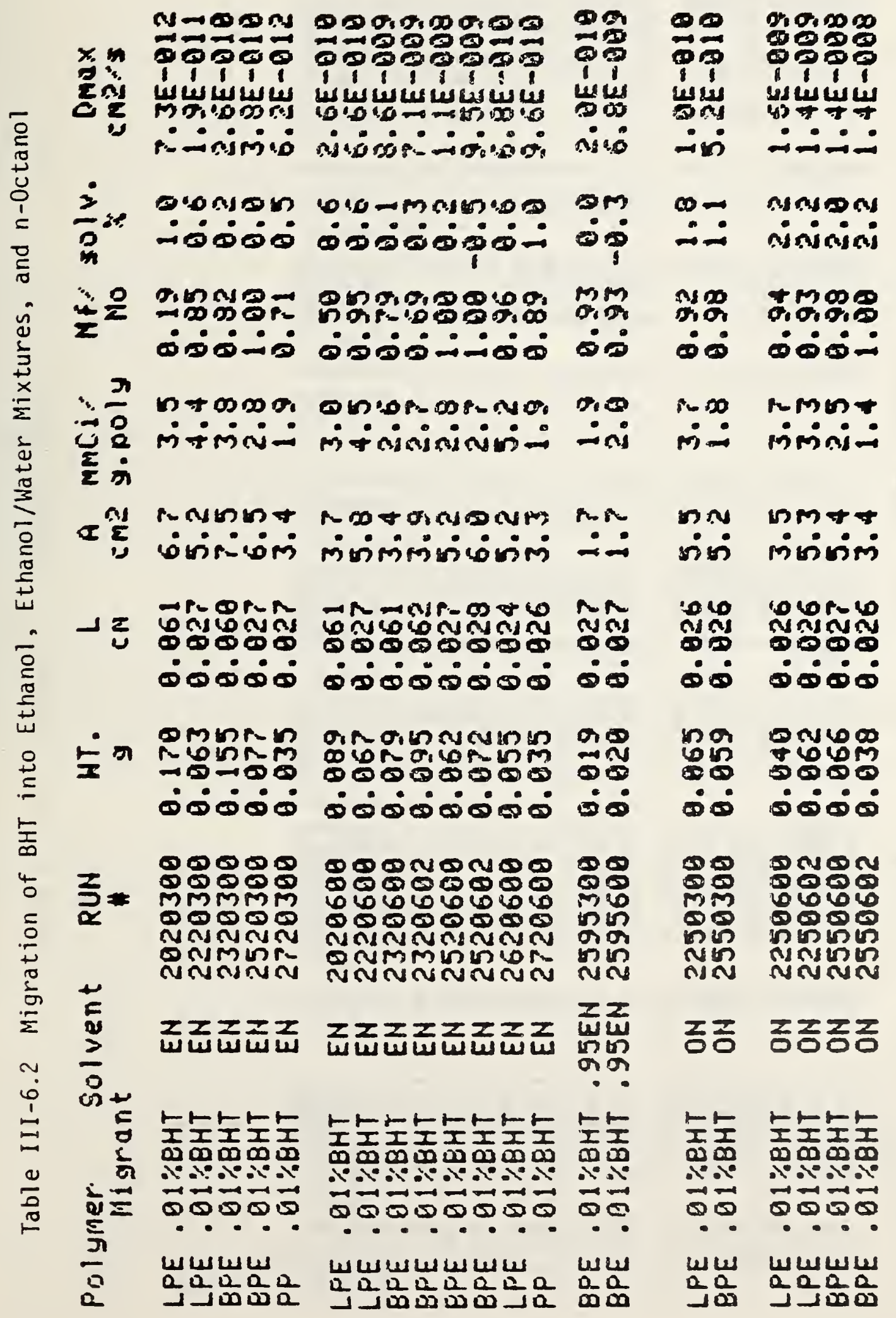


00000000000 000000

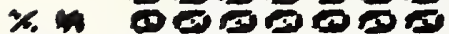
$2 \%$

zar misurenters

e 2 corsisonat: - cirisini-

$\div \quad 00000000$

20.

n

*D aspertorisos

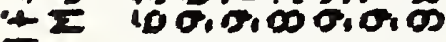
$z$

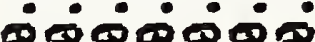

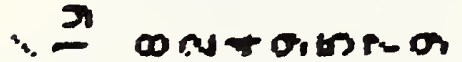

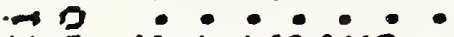

1) $2+\pi m n i n-$

E.

$\Sigma$

a d $\rightarrow 00$ Mr w

$\sigma=0 \cdot 0 \cdot 0$

fonmingm

Or-prNwero

- I oninionisu

- 0000000

ம

- nmonnmo

- $\Rightarrow$ cortriont

$-000000$

i- 00000

- $\quad-0 \div-10=$

J. MMMNMMM

c 0000000

MMMMMMM

oncumisin

जलMNWNA

$+$

E

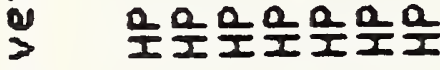

0

os

E $-1-1-1-1$

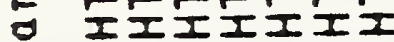

L 00000000

क

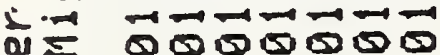

$0=00000000$

แथाजน

2. 2 a 2 a $1 \pm 10000$

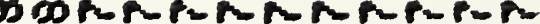

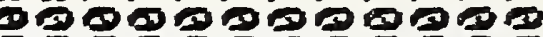
45 $1,1,1,1,1,13$

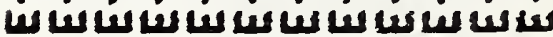

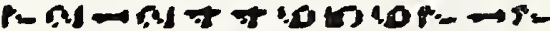
í - imrinimi-

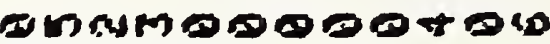
ம்

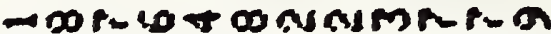

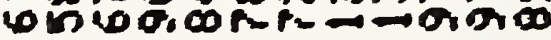

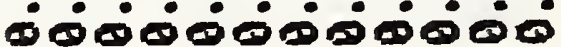

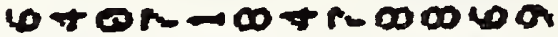

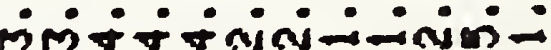

$N$ tormugnat

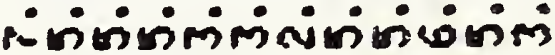

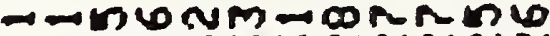

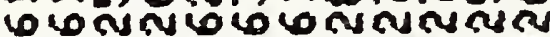
000000000000

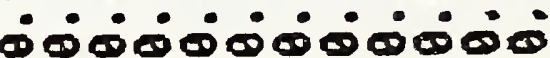

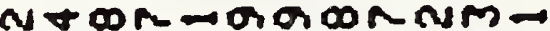
om - -0000000000 $\because \dot{0} \dot{0} \dot{0} \dot{0} \dot{0} \dot{0} \dot{0} \dot{0}$

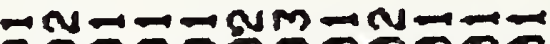
000000000000 6.

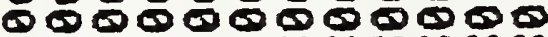
MMMMMMMMNMNM $00-\mathrm{NmMM}+106 \mathrm{~N}$

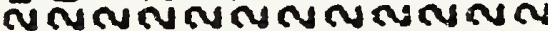

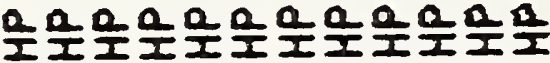

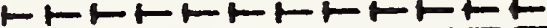
젇ㅈ도도도

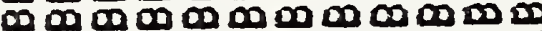

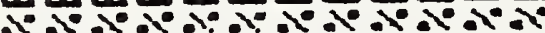
$-1-1-1-1-10-100$

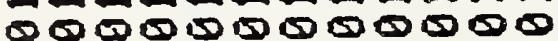

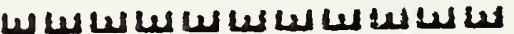

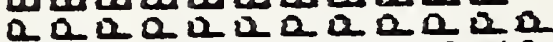

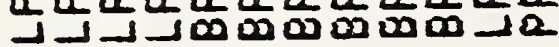




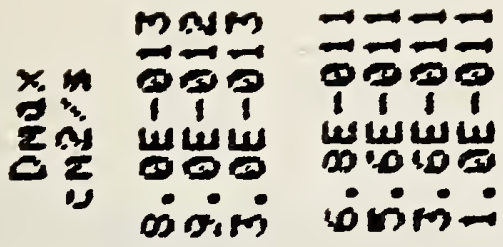

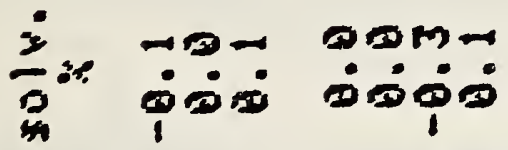

$$
\begin{aligned}
& \text { * } 2 \text { M⿻上丨 } \\
& \text { z } \dot{0 i s} \dot{0} \dot{0} \\
& \text { d } \\
& \text { 茥 } \\
& \text { - } \bar{\varepsilon} \dot{1}
\end{aligned}
$$

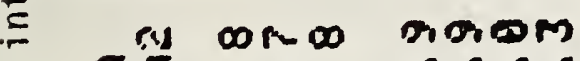

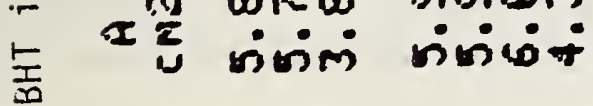

$$
\begin{aligned}
& \text { to } \infty N n \text { rom }
\end{aligned}
$$

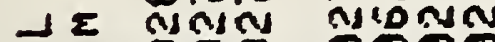

$$
\begin{aligned}
& 0000 \\
& \text { ن்க }
\end{aligned}
$$

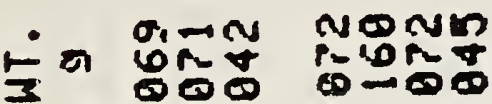

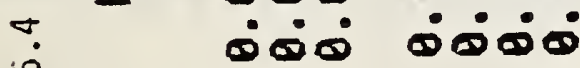

$$
\begin{aligned}
& \stackrel{i}{=} \quad 00000000 \\
& \text { z } 0000000 \\
& \text { Z }=\text { MMm } \\
& \text { क } \\
& \text { uiñ Nmin } \\
& \text { MNO NONA }
\end{aligned}
$$

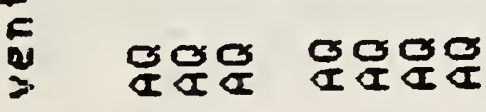

$$
\begin{aligned}
& \text { in }
\end{aligned}
$$

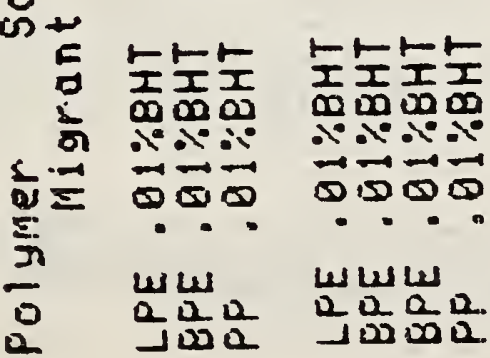


TMmM

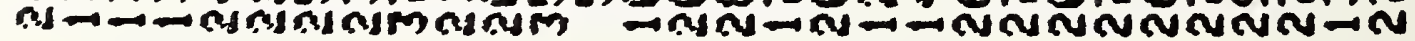

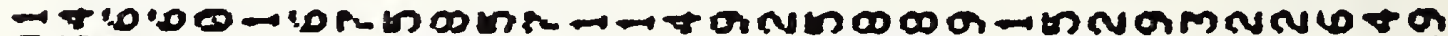

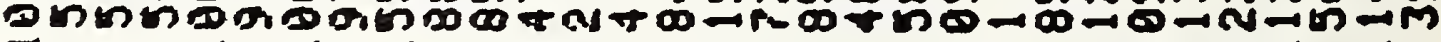

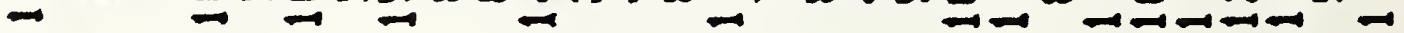
$\Upsilon$

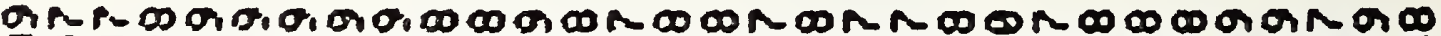

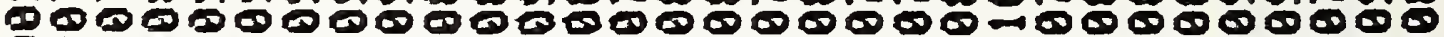

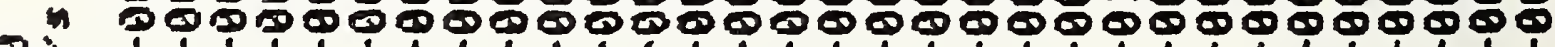
$\begin{array}{llllllllllllllllllllllllllllllll} & 1 & 1 & 1 & 1 & 1 & 1 & 1 & 1 & 1 & 1 & 1 & 1 & 1 & 1 & 1 & 1 & 1 & 1 & 1 & 1 & 1 & 1 & 1 & 1 & 1 & 1\end{array}$

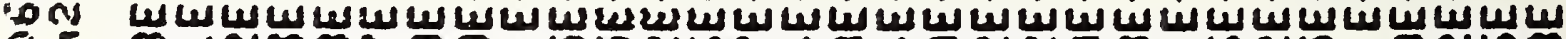
C.

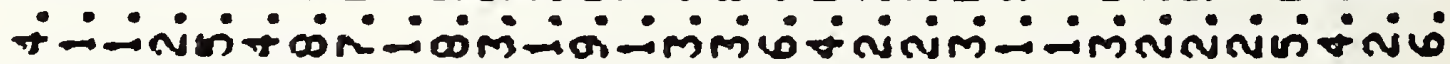

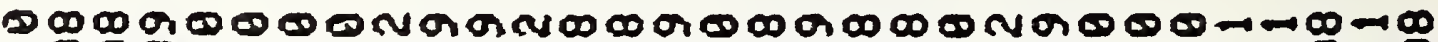

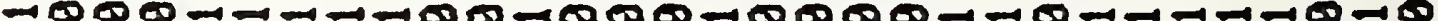

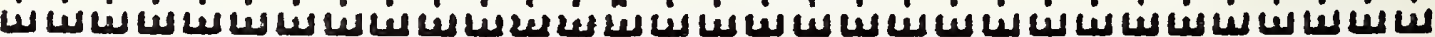

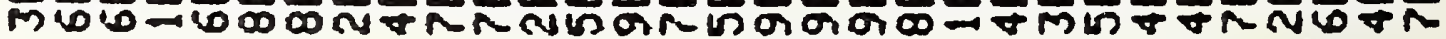
-

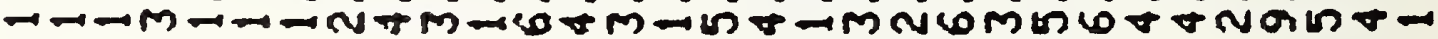

$2=$

00000000000000000000000120001000000

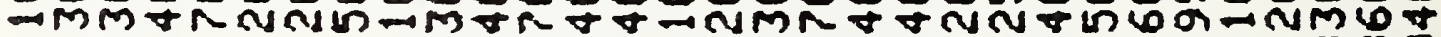

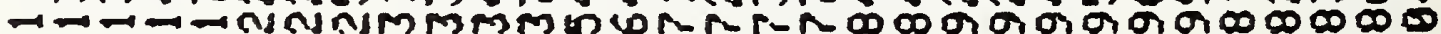
NतNMN

$\frac{1}{2}$ des (ภ)

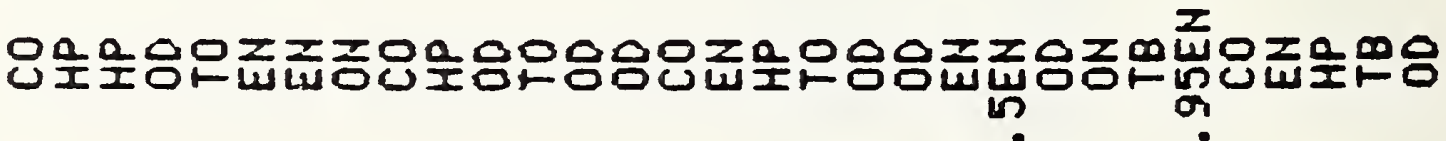

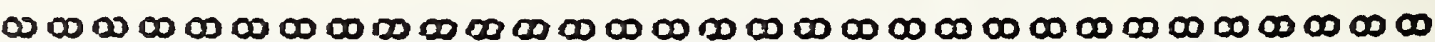

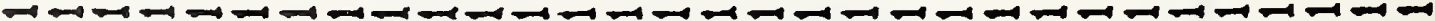

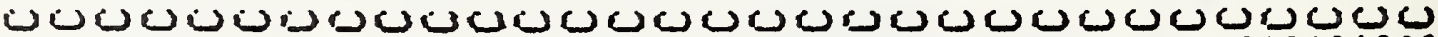

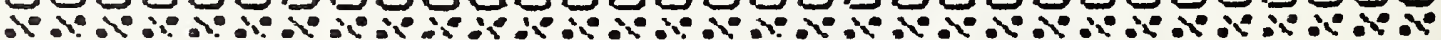

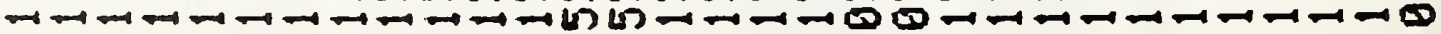
$0 \infty \pi$

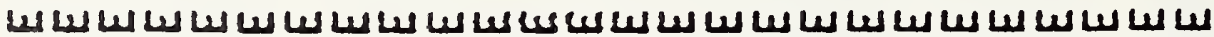

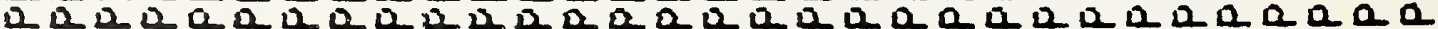

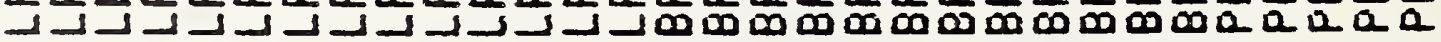




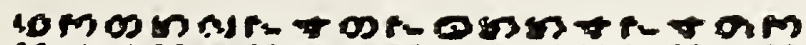

w-

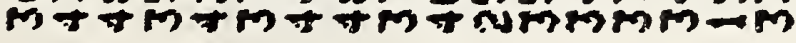

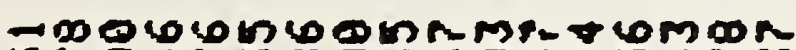

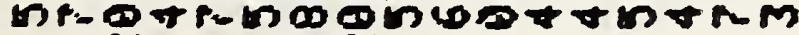

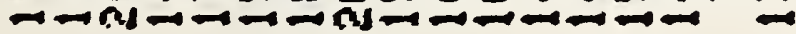

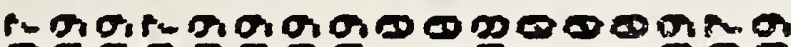

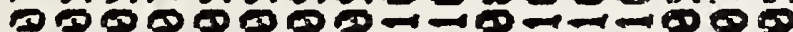
09000000000000000 $0 \div, 1,1,1,1,1$, 1 , 1

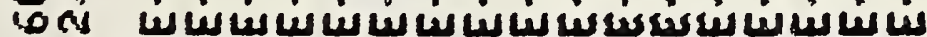

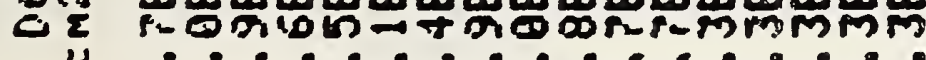

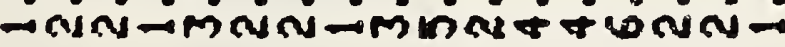

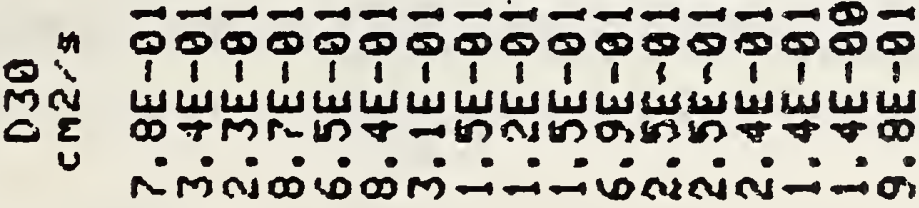

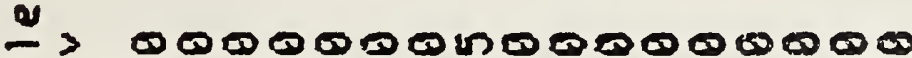

- manmingr.

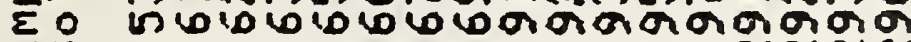

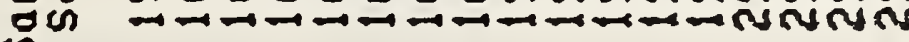
(

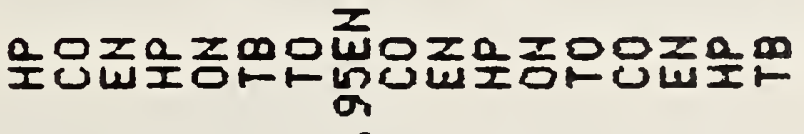

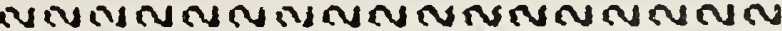
МММММММММММММММММ

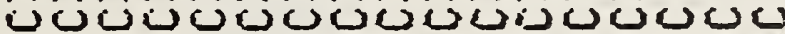

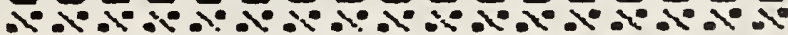
$\rightarrow$ $\rightarrow$ -

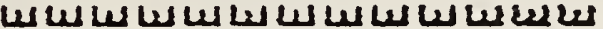

$\frac{2}{2}$

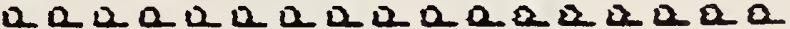

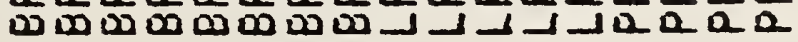




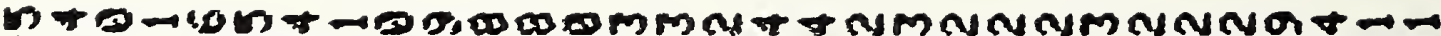

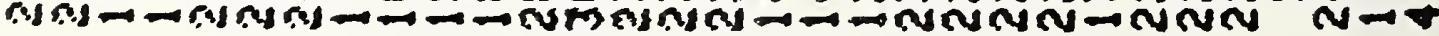

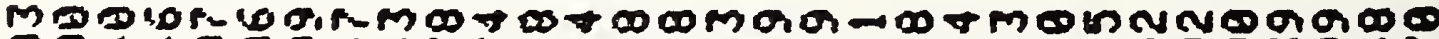

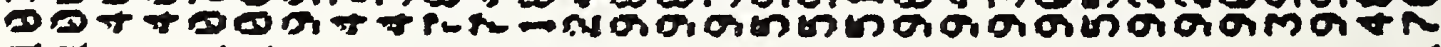
$\rightarrow \rightarrow \infty$

42

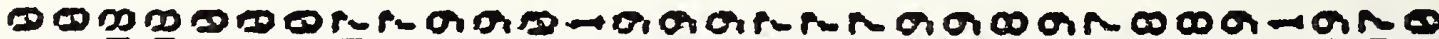

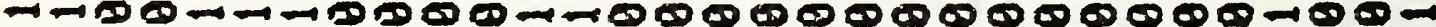

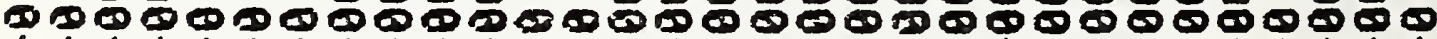

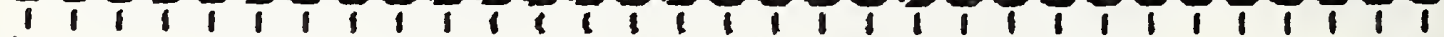
"4

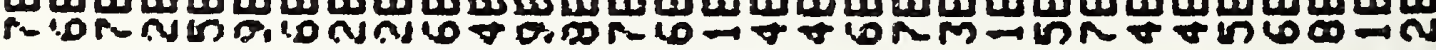

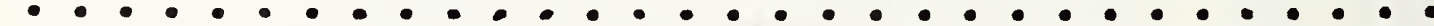

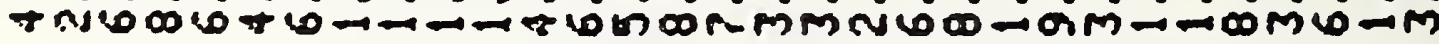

and

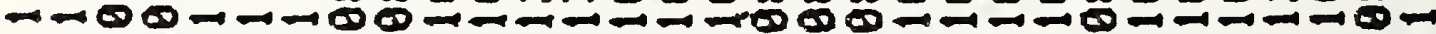

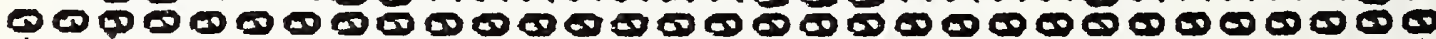

mis

$0 \Sigma$

4

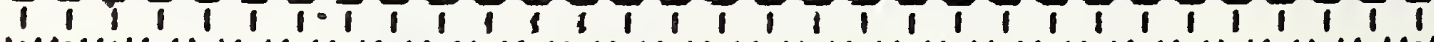

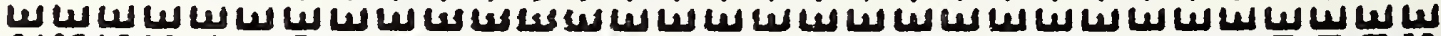
जल M०

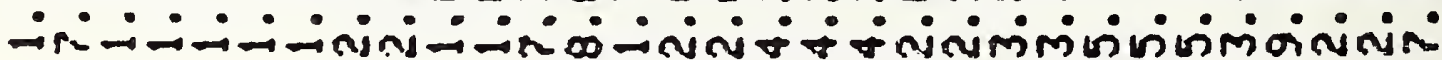

(1)

13

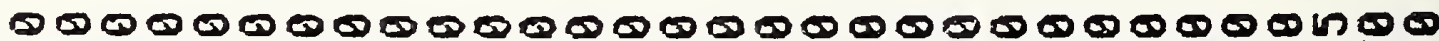
- CIMMr- MIMmLU

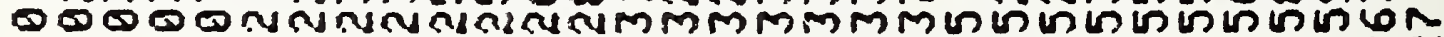

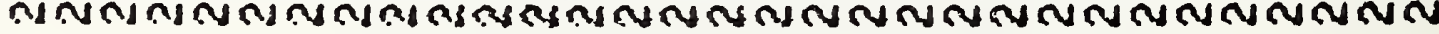

0 I

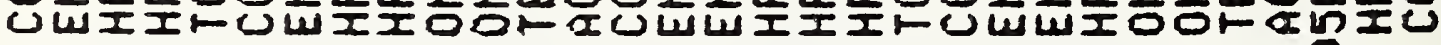

os

$+$

-

8

2

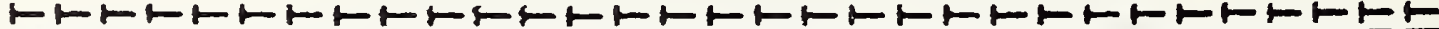

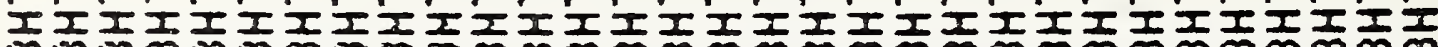

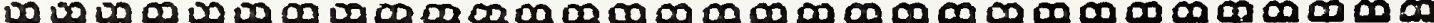

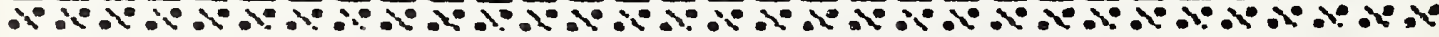

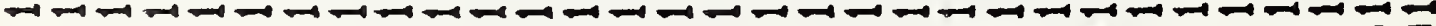

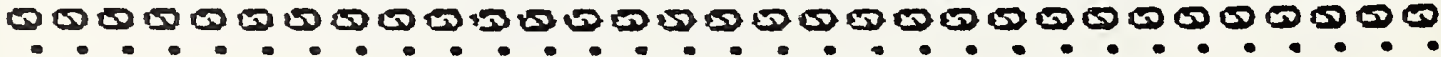

7

5

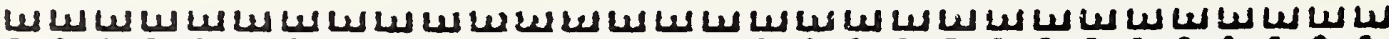

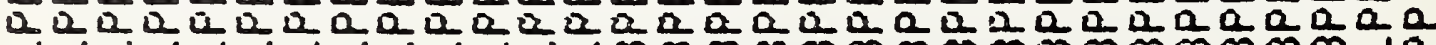
1 


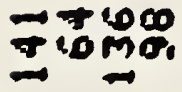

3NO-

$-0-1$

900

0
0
0
0
0
0
0

ulusus

of-Dos

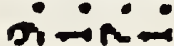

Non

$-\infty=1$

000

1111

un

जr

ம-inm

$\frac{1}{0}$

0000

OMDO

rrró

푼온뭄

ヒレレト

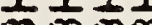

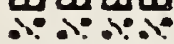

무요

0000

믐음뭉 


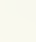


may be used as the starting value in a reiterative numerical computation.

Equation (1) converges rather slowly, at small value of $T$, thus a sufficient number of terms must be used to avoid premature termination of the computation. At $T \ll 1$, approximately $3^{\left(-\log _{10} T\right)}$ terms are required to reach a reasonable precision. The following approaches may be used to simplify the computational effort depending on the conditions and ranges of applications. Simplified Computation

At small values of $\alpha(<<1)$, a master curve of the quantity $M_{t} /(1+\alpha) M_{\infty}$ s which is equal to $M_{p t} / M_{\text {so }}$ for absorption or $M_{s t} / \alpha M_{p o}$ for extraction, as a function of $T / \alpha^{2}$ may be constructed from equation (1). However, by 1 imiting the computations to $0.1>T>0.001$, the number of terms required may be 1 imited to about 5 at T 0.1 and about 45 at T 0.001 for reasonable accuracy. The values of $M_{t} /(1+\alpha) M_{\infty}$ at small $\alpha$, but at the same $T / \alpha^{2}$, are equal to the values calculated for large $\alpha$. The results of this selective computation deviates from the more rigorous and tedious computations by about $0.001 \%$ at T 0.1 , and much less than $0.001 \%$ at 1 ower $T$ values.

The diffusion process may be divided into three regions of $T$ :

Region I, $T<0.1$. The system is far from equilibrium, $M_{t} /(1+\alpha) M_{\infty}$ is a function of $T / \alpha^{2}$ only.

Region II, $5>T>0.1$. The system is approaching equilibrium and detailed computation must be carried out for different a values (only 2 to 6 terms are required for equation (1) in this region). For $\alpha<1$ and $T / \alpha^{2}>10^{3}, M_{t} /(1+\alpha) M_{\infty}=1-\alpha /(\pi T)^{1 / 2}$ before reaching equilibrium.

Region III, T>5. For all practical purposes, equilibrium has been reached with the deviation $\delta=1-M_{t} / M_{\infty} \leq 10^{-T}$, where $M_{\infty}=M_{S O} /(1+\alpha)$ for 
the case of absorption and $M_{\infty}=M_{p o} \alpha /(1+\alpha)$ for the case of extraction. For $\alpha<1$, equilibrium may be reached much earlier.

Computation for Migration into Infinite Media

When $\alpha \rightarrow \infty, M_{t} / M_{\infty}$ approaches a limit, and equation (1) can be reduced to a function of $T$ only: $\quad M_{t} / M_{\infty}=1-2 \sum_{n=1}^{\infty} \frac{1}{q_{n}^{2}} \exp \left(-q_{n}^{2} T\right)$ where $q_{n}=(n-1 / 2) \pi$. For $T<0.1, M_{t} / M_{\infty}$ is a 1 inear function of $T^{1 / 2}$.

$$
M_{t} / M_{\infty}=2(T / \pi)^{1 / 2}=1.128379 T^{1 / 2}
$$

Deviations of equation (3) from equation (2) is less than $10^{-10}$ at $T<0.05$, about $10^{-6}$ at $T=0.1$, about $5 \times 10^{-4}$ at $T=0.2$ and becomes much greater than 0.01 at $T=0.3$ or higher.

Alternate Approximation

An alternative form of the solution [7]

$$
M_{t} / M_{\infty}=(1+\alpha)\left[1-e^{T / \alpha^{2}} \operatorname{erfc}\left(T^{1 / 2} / \alpha\right)\right]
$$

may be used in some cases and is relatively simple to compute. One of the rational approximations ${ }^{[8]}$ for the error function yields the following

$$
M_{t} /(1+\alpha) M_{\infty}=1-\sum_{n=1}^{5} a_{n} \tau^{n}+\varepsilon
$$

where $\tau=l /\left(1+0.3275911 T^{l / 2} / \alpha\right), a_{1}=0.254829592, a_{2}=-0.28449636, a_{3}=1.421413741$, $a_{4}=-1.453152027, a_{5}=1.061405429$, and $|\varepsilon|<\omega 1.5 \times 10^{-7}$.

At $\alpha<<1$, results computed from equation (2) or (3) deviates less than 0.0001 from that of equation (1) at $T / \alpha^{2}<5$ or at $M_{t} /(1+\alpha) M_{\infty}<0.75$. Maximum deviation of 0.0035 occurs at $T / \alpha^{2} \sim 500$ or at $M_{t} /(1+\alpha) M_{\infty} \sim 0.97$. Therefore, equation (4) or (5) may be used to generate the master curve for equation (1) at $T<0.1$ (Region I). However equations (4) or (5) do not yeild any information about the region approaching equiltrium at larger values of $\alpha$, and hence should be used with discretion. 
By combining Equation (4) or (5) at $T<0.1$ (Region I) and

Equation (1) at $T>0.1$ (Region II and III), computational requirements for the solution of the diffusion equation between a plane sheet and a well stirred liquid may be reduced to a minimum.

Diffusion coefficient at any point may be estimated from the above computation method by means of regression.

Effect of Swelling

The diffusion coefficients in the tables are calculated based on the original measured thickness of the sample, which is also given in the tabies. The final sample is usually swollen with solvent. For volatile solvents, the absorbed solvent will leave the polymer soon after the sample is taken out of the extracting solvent. In these cases, the final sample weight is determined immediately after the surface of sample has been wiped free of the solvent. The dimensions of the final sample are generaily not measured, however they may be estimated from the amount of soivent absorption by assuming an isotropic expansion. The diffusion coefficient may be adjusted to reflect the increase in the thickriess as follows:

$$
\begin{aligned}
D & =D_{0}\left(\frac{L}{L_{0}}\right)^{2}=D_{0}\left(\frac{V}{V_{0}}\right)^{2 / 3} \\
& =D_{0}\left[1+\frac{\delta_{f}}{1-\delta_{f}} \cdot \frac{\rho_{0}}{\rho_{s}}\right]^{2 / 3}
\end{aligned}
$$

where $\delta_{f}=\left(W_{f}-W_{0}\right) / W_{f}$. $D, L, V, M$ and $p$ are the diffusion coefficient, thickness, volume, weight and density respectively. The subscripts 0 , $f$, and $s$ denote the original polymer, final polymer and solvent respectively. The sum of fractional weight increase $\delta_{f}$ and the fractional decrease in weight due to the migrant loss is given also in the table and indicates the weight percent of solvent content in the final sample. Therefore, the diffusion coefficients based on the final sample thickness 
are increased from those based on the original thickness by approximately the same order of magnitude as the increases in weight. 
Table II -1

Characteristics of Polyolefin Samples

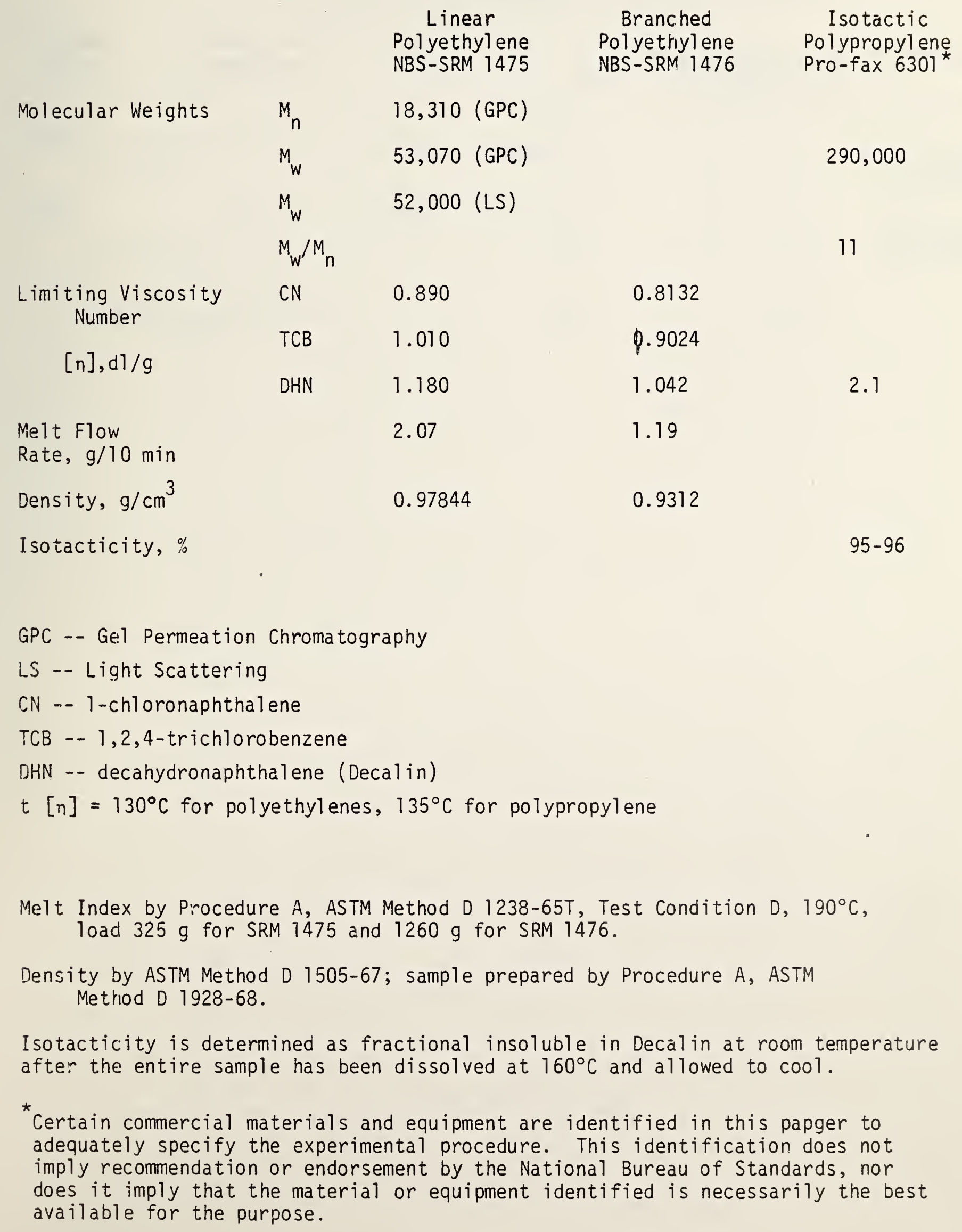


Table II-2

Characteristics of Ethyl ene-Vinyl Acetate Copolymers

Designation

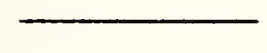

Petrothene NA294-00

UI trathene UE657-00

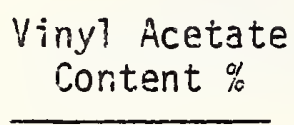

5.1

13.4

\begin{tabular}{ccc}
$M_{n}$ & $M_{n}$ & Melt Index \\
- & - & \\
\hline
\end{tabular}

15000

89300

$18700 \quad 103000$
1.9

0.46

0.935
Density $\mathrm{g} / \mathrm{cm}^{3}$

0.925 
Table II-3

Characteristics of Radioactive Tracers

$\begin{array}{lcc} & \frac{\mu C_{i} / m g}{n g / 25 \mathrm{dpm}} \\ n-\mathrm{C}_{18} \mathrm{H}_{38}-1-{ }^{14} \mathrm{C} & 86.0 & 0.13 \\ \mathrm{n}-\mathrm{C}_{32} \mathrm{H}_{66}-16,17-14 \mathrm{C} & 13.3 & 0.83 \\ \begin{array}{c}3,5-\text { di-tert-butyl-4 } \\ \text { hydroxytoluene-7-14 C } \\ \text { (BHT) }\end{array} & 45.5 & 0.25 \\ & 57.9 & 0.19\end{array}$


Table II -4

$$
\begin{gathered}
\text { Solvent } \\
\times 10^{3} \\
\hline
\end{gathered}
$$

10, Corn 0 il (CO)

20, Ethanol

(EN)

21, $10 \%$ Ethanol

$(.1 \mathrm{EN})$

(.3 EN)

23, 30\% Ethanol

25, 50\% Ethanol

(.5 EN)

27, 70\% Ethanol

(.7 EN)

29, 90\% Ethanol

30, n-Heptane

40, n-Octadecane

50, n-Octanol

60, Tributyrin

70, Trioctano in

80 , Water

90, HB307

Synthetic Triglyceride Mixture

95, 95\% Ethanol (.95 EN)
Temperature

$\times 10^{1}$

$24,24^{\circ} \mathrm{C}$

$30,30^{\circ} \mathrm{C}$

$40,40^{\circ} \mathrm{C}$

$60,60^{\circ} \mathrm{C}$
Method

$\times 10^{0}$

0, Limited Solvent Volume

1, Unlimited Solvent Volume

2-8, Dupl icate (or Renewed Extractions)

9, Absorption Experiment

(A)

Letters in paratheses are abbreviations used in tables. 
Table II-5

Nominal

Sample Code $\times 10^{5}$

1

2

3

4

5

6

7

8

9

11

13

14

15

16

17

18

19

20

21

22

23

24

25

26

27

28

29

30

31

32
Polymer

LPE

LPE

LPE

LPE

LPE

LPE

BPE

$B P E$

BPE

LPE

BPE

LPE

BPE

BPE

BPE

LPE

LPE

LPE

LPE

LPE

BPE

BPE

$B P E$

LPE

$P P$

$P P$

$P P$

$P P$

$E-5 \%$ VA

$\Xi-13 \%$ VA
Migrant

C18

C18

C18

$\mathrm{C} 18$

C18

C18

$\mathrm{C} 18$

C18

$\mathrm{C} 18$

C18

C18

C18

C32

C32

$\mathrm{C} 32$

C32

C32

BHT

BHT

BHT

BHT

BHT

BHT

BHT

BHT

C18

C32

C18

BHT

BHT
Migrant Conc., \%

0.64

0.63

0.013

0.01

5.2

5.5

0.85

9.8

0.85

0.009

0

0

1.86

2.26

0

0

1.0

0.0062

0.0069

0.0072

0.0052

0.0030

0.0045

0.0095

0.0029

0.82

0.97

6.8

0.0091

0.0057 $\frac{\text { Sp. Act. }}{\mu \mathrm{Ci} /}$

g. Polymer

2.1

2.2

1.6

0.96

0.14

0.13

0.020

0.23

0.17

1.1

93.

113.

5.2

3.6

4.0

4.2

3.0

1.8

2.6

5.5

1.8

4.9

5.3

0.16

5.3

3.3
Thickness

$\mathrm{cm}$

0.072

0.019

0.070

0.020

0.084

0.027

0.064

0.066

0.057

0.263

0.064

0.064

0.064

0.028

0.059

0.060

0.025

0.027

0.061

0.028

0.027

0.025

0.027

0.027

0.027

0.028

0.029

0.069 
i. Sanchez, I. C., Chang, S. S., McCrackin, F. L. and Smith, L. E., "An Evaluation of Existing Models Describing the Migration of Additives in Polymers," NBSIR 78-1499, 1978.

2. Smith, L. E., Sanchez, I.C., Chang, S. S. and Mccracking, F. L., "Models for the Migration of Paraffinic Additives in Polyethylene," NBSIR 79-1598, 1979.

3. Smith, L. E., Sanchez, I. C., Chang, S. S., McCrackin, F. L., and Senich, G. A., "Models for the Migration of Additives in Polyolefins," NBSIR 79-1779, 1979.

4. Smith, L. E., Chang, S. S., McCrackin, F. L., Sanchez, I. C. and Senich, G. A., "Models for the Migration of Additives in Polyolefins," NBSIR 80-1999, 1980.

5. Smith, L. E., Chang, S. S., Mccrackin, F. L., Senich, G. A. and Wang, F. W., "Models for the Migration of Low Molecular Weight Additives in Polyolefins," NBSIR 81-2264, 1981.

6. Smith, L. E., Chang, S. S. and Senich, G. A., "Migration of Low Molecular Weight Additives in Polymers," NBSIR 81-2314, 1981.

7. Crank, 3. "The Mathematics of Diffusion," Oxford University Press, 1975.

8. Abramowitz, M. and Stegun, L. A., "Handbook of Mathematical Functions," AMS 55, National Bureau of Standards, GPO, 1964. 


\section{Results of Polyolefin Extraction Experiments}

The results of extraction experiments are summarized in Table III.l through III.3 for the extraction of n-octadecane, n-dotriacohtarie and BiT by triglycerides, ethanol, n-octanol, ethanol/water mixtures, $n$-heptane, n-octadecane, and water.

The tables provide information on the test pieces as to their weight, thickness $L$, exposed area $A$, specific activity of polymer, amount extracted at the end of experiment $M_{f} / M_{0}$, the amount of solvent retained in the final sample and the observed maximum of the diffusion coefficient.

The value for $M_{f} / M_{0}$ in the tables is the final fraction of total additives migrated at the end of the experiment. It may be either an equilitrium value or a non-equilibrium value after a long time ( 6 months to cne year) lapse. In the case of BHT experiments, most of the $M_{f}$ values for migration into good solvents denote the available fraction of BHT in the sample, which varies with the conditions underwhich the sample was prepared. Therefore, in calculating diffusion coefficients for samples containing BHT, an adjusted $M_{0}$ is assigned to each sample according to the equilibrium $\left(M_{f} / M_{0}\right)$ values observed in the good solvents. For the case of $n-\mathrm{C}_{32} \mathrm{H}_{66}$ in polypropylene or other samples which exhibit surface blooming of migrants, the total amount of migrant is a? so adjusted for the amount of migrant on the surface, before diffusion coefficients calculating from it.

Tables III-1 through III-3 summarize the experimental results arranged by migrant or additive and then according to the type of polymer, additive concentration, sample thickness, and, finally, solvents and temperature. In order to provide easy cross-references, Tables III-4 
through III-6 sumarize the experimental results which are arranged first by solvent and then by additive and other parameters.

The activation energies, E, calculated by a simple Arrhenius equation:

$$
D=A \exp (-E / R T)
$$

are given in Table III-7. 


\section{Loss of BHT from Polymers in Ambient Atmosphere}

$A D L$, Inc. had reported through private communications concerning the high volatility of BHT from their samples. When we examined our observed total BHT contents from various experiments over a $11 / 2$ year period on a branched polyethylene sample, code 25 , containing $45 \mathrm{ppm}$ of BHT, we found a rather noticeable trend of decreasing radioactivities as a function of starting date of the experiment, Figure IV-1, al though the sample plaque was always wrapped in aluminum foil except during short periods when pieces were cut from the plaque.

The volatility of BHT is further indicated by the following experiment. A piece of $E-13 \%$ VA copolymer containing $60 \mathrm{ppm}$ of BHT (sample code 32), $61 \mathrm{mg}$ in weight with about $2 \mathrm{~cm}^{2}$ of surface area, was placed in a sealed $20 \mathrm{ml}$ vial. The bottom of the vial was kept at $30^{\circ} \mathrm{C}$ and the vial cap at $22-23^{\circ} \mathrm{C}$. After 63 hours it was found that the aluminum cap liner had a radioactivity of $0.51 \%$ of the test piece and the glass wall of the vial had a radioactivity of $0.95 \%$ of the test piece. Thus in a relatively short period, $1.5 \%$ of the BHT was sublimed from the test piece at rather low temperatures in a closed vessel.

A further test was performed on both sample plaques 25 and 32 . Four pieces were cut from each of the sample plaques. These four pieces were subjected to the following tests:

(A) The test piece was dissolved in $0-x y$ lene to check the current specific activity.

(B) The test piece was extracted immediately by ethanol at $60^{\circ} \mathrm{C}$ for three days. The residual specific activity and the activity of the extract were recorded.

(C) The test piece was suspended in a ventilation hood at room temperature for three days before procedure (B) was performed. 
(D) The test piece was placed in a vial. Forced air was blown into the vial for three days at room temperature before procedure (B) was performed.

The results of these tests are presented in Table II-1. The test pieces $A$ and $B$ were used as controls. Apparently 30 to $50 \%$ BHT is lost from thin sheets of BPE or E-VA copolymer in mobile air at room temperature for three days. The specific activity of sample code 25, BHT in BPE, at the time of these tests was only $50 \%$ of the original specific activity of $2.8 \mu \mathrm{Ci} / \mathrm{g}$ polymer, determined 16 months earlier.

These experiments and our prior experience indicate that: (1) the amount of additive remaining in the polymer after processing to produce plaques is a fraction of the additive mixed into the raw polymer, for certain reactive additives, only a fraction of additive in the polymer may be extractable, the rest may be chemically bound to the polymer, (3) the total activity of the test pieces should be checked for each experiment not only to measure the uniformity of the additive concentration but also to measure the change in additive content as a function of time. Therefore reports which the results are normalized to the amount of additive supposely added to the polymer or to the total amount of additive present in the polymer when it was manufactured, without paying further attention to the amount of additive either reacted or lost, may yield seriously incorrect kinetics of migration. 


\section{Table IV-1}

Sample Code Test Piece $\begin{gathered}\text { Specific Activities, } \mu \mathrm{Ci} / \mathrm{g} \text { polymer } \\ \text { Residue Total }\end{gathered}$

25

BHT in BPE

32

BHT in E-13\% VA
A

B

C

D

A

B

C

D

1.28

0.042

0.053

0.052

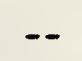

0.077

0.081

0.068
1.30

.93

.89

3.29

3.45

2.18

1.49
Percent of Origina? Activity (Average of $A+B$ )

100

100

72

69

100

100

65

44 


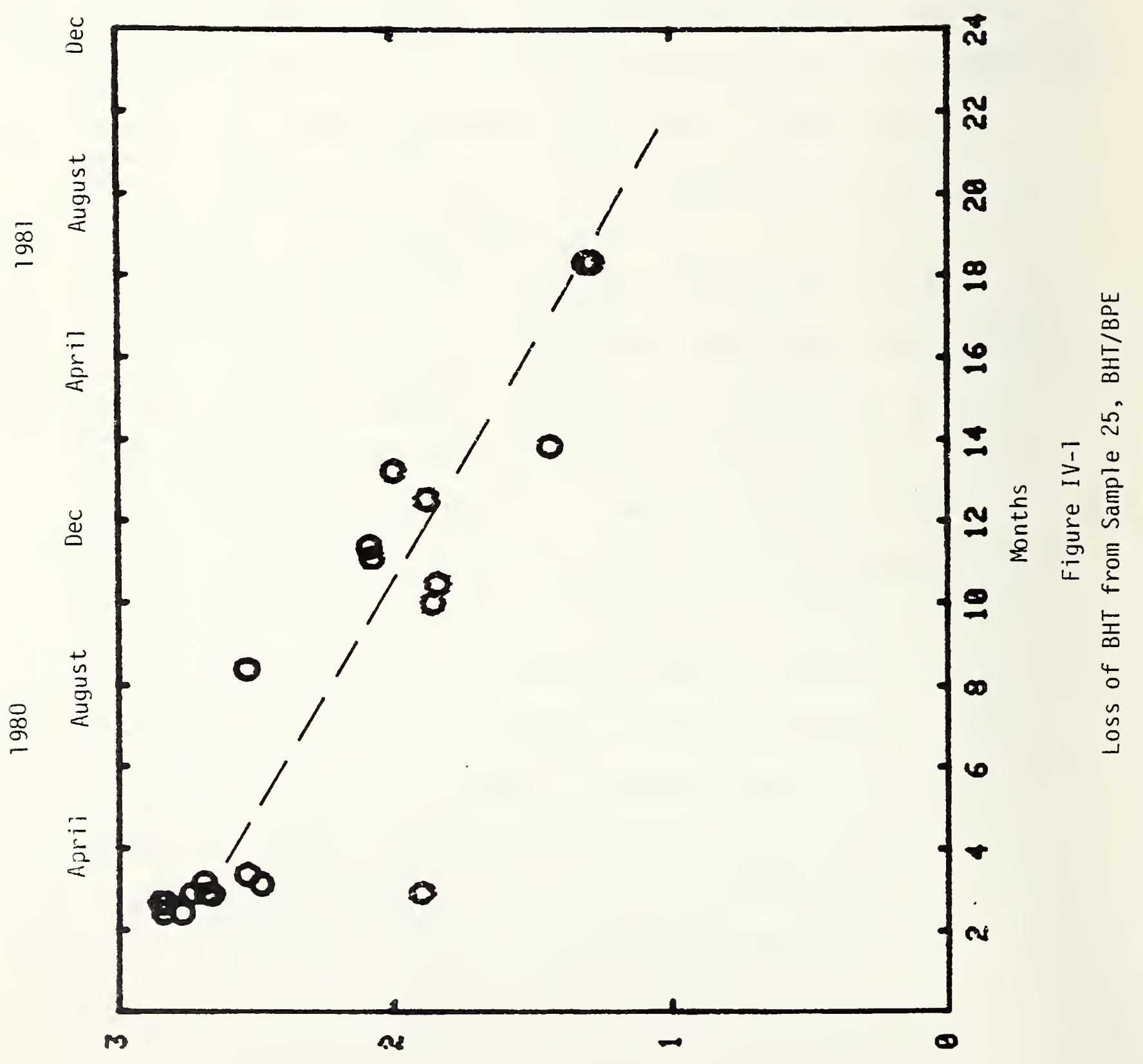

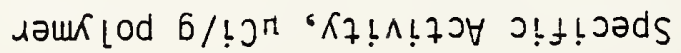


V. Migration of BHT from Ethylene-Vinyl Acetate Copolymers

Ethylene-viny? acetate (E-VA) copolymers of relatively low vinyl acetate contents have been used in increasing quantities in recent years as food-contact polymer films. The migration of the antioxidant BHT from two copolymers having 5 and 13\% vinyl acetate content into corn 0i1, anhydrous ethanol, 95\% ethanol, water and $n$-heptane was observed with ${ }^{14} \mathrm{C}$-labeled BHT. The characteristics of these polymers were shown earlier in Table I-2. The characteristics of the additive BHT were presented earlier in Table I-3. The sample plaques were prepared in similar manner to those of polyethylene and polypropylene samples described earlier, except that lower molding temperatures were used for the E-VA copolymers. The E-5\% VA and E-13\% VA samples were designated as plaque 31 and 32 with thickness of 0.027 and $0.068 \mathrm{~cm}$ and BHT contents of 9 and 57 PPM, respectively.

The results for individual extraction experiments are summarized in Table V-1. E-VA copolymers absorb a fair amount of solvents as shown in the table. The listed values are fractional weights of solvent contained in the final sample. Thus for experiment 3230600 , the final sample contained n-heptane amounting to about $120 \%$ of the original weight of the polymer. The $12 \%$ corn 011 content for sample 3210600 is higher than all other corn oil experiments.

The results of all extraction experiments for individual E-VA copolymers are shown graphically in Figures $V-1.1$ through $V-1.4$ for the migration of $B H T$ from $E-5 \%$ VA at 30 and $60^{\circ} \mathrm{C}$, and from $E-13 \%$ VA at 30 and $60^{\circ} \mathrm{C}$, respectively. There are three illustrations for each figure, i.e., (A) $M_{t} / M_{0}$ is plotted against $t / L^{2}$ in a $\log$ - $\log$ plot to indicate the migration behavior over several orders of magnitudes in both $M_{t} / M_{0}$ and time, (B) $M_{t} / M_{0}$ is plotted against $t^{1 / 2} / L$ for the short time region 
to indicate the behavior observed during fast extractions, and (C) $M_{t} / M_{0}$ is plotted against $t^{1 / 2} / L$ for the entire length of time of experiments.

From both Tables $V-1$ and Figures $V-1$, the residual BHT content in the $5 \%$ vinyl acetate copolymer samples, code 31 , at the end of extration are seen to vary from 7 to $12 \%$. It is estimated from graphical extrapolation that approximately $7-8 \%$ of the original BHT content is not extractable even by good solvents, such as n-heptane. Almost all of the BHT content in the $13 \%$ vinyl acetate copolymer is extractable. The $5 \%$ water content in $95 \%$ ethanol has no noticeable effect on the BHT extractability for the copolymers. No partitioning of BHT was observed between the copolymers and solvents such as $n$-heptane, corn oil, anhydrous ethanol and $95 \%$ ethanol, due to the near-complete of extraction of all available BHT from the copolymers by these solvents.

Figures V-2.1 through 5 show the migrational behavior of BHT from E-VA copolymers into various solvents, n-heptane, corn oil, ethanol, 95\% ethanol and water. These illustrations offer comparisons of behavior among different E-VA copolymers and/or at different temperatures of extraction:

In a more concise comparison of this behavior, the apparent maximum diffusion coefficients of the swollen polymers are listed in Table V-2 together with the activation energies for diffusion. In all cases except water, the diffusion coefficient for the $13 \%$ vinyl acetate copolymer is about 2 to 3 times greater than that for the 5\% copolymer. Increasing in the temperature from 30 to $60^{\circ} \mathrm{C}$ generally increases the diffusion coefficient by 10 to 20 fold, although the changes for the solvent $n$ heptane are only on order of 4 to 6 .

The diffusion coefficients in $95 \%$ ethanol are only slightly less than those for anhydrous ethanol, about $10-15 \%$ less for $5 \%$ vinyl acetate copolymers and about $25-45 \%$ less for $13 \%$ copolymer. Both diffusion 
coefficients for experiments in anhydrous ethanol and $95 \%$ ethanol are greater than those for corn oil by similar magnitudes.

The migrational behavior of BHT from the two E-VA copolymer into water is rather unusual, as can be seen in figure $V-2.5$. The $\log \left(M_{t} / M_{0}\right)$ versus $\log \left(t / L^{2}\right)$ representation in Figure $V-2.5$ is changed for Figures $V-3 . B$ and $V-3-C$ into the more familiar $M_{t} / M_{0}$ versus $t^{1 / 2}$ representation. Figure $V-3-B$ is the short-time portion of Figure $V-3-C$ which covered the entire time scale of experiment. These results indicate that the migration of BHT from E-VA copolymer into water occurs in two distinct stages, and that in contrast to the observations for other solvents, the diffusion coefficients of BHT from E-13\% VA copolymer are slower than those from E-5\% VA copolymer in the first stage. In the second stage the diffusion coefficients from E-13\% VA are apparently approaching those from E-5\% VA and can even surpass the latter when the time of experiment is lengthened to allow equilibrium extraction.

In the first stage, $10-50$ hours, approximately $15 \%$ of the BHT from E.5\% VA copolymer and about $1 \%$ of the BHT for E-13\% VA copolymer, were extracted by water before the release of BHT levels off into a second stage behavior. It has not been determined whether the second stage of extraction, with its distinctiy different diffusion coefficient, is due to the distribution of BHT in phase-separated regions of the copolymers or due to the reaction of water and BHT over prolonged periods.

Another plausible explantion for the two stage behavior would be surface blooming, or a very high migrant concentration near the surface layer, although the time lapse of 10 to 50 hours seems to be longer than usually needed to remove migrant from the surface or surface layer. By extending the second stage behavior to $t=0$, one may estimate from 
Figure $V-3-B$ that there might exist surface blooming of BHT amounts to about 7.5 and $11 \%$ for the E-5\% VA copolymer and 0.6 and $0.57 \%$ for the E-13\% VA COpolymer, for 30 and $60^{\circ} \mathrm{C}$ experiments, respectively.

The diffusion coefficients presented in Table $V-2$ were calculated by first adjusting the original loading, $M_{0}$, to the available BHT, $M_{0}^{*}$, when necessary. The first stage diffusion coefficients were calculated based on the above mentioned percentages for $M_{\infty} / M_{0}^{*}$. By substracting the first stage $M_{\infty}$, normalizing and then applying equilibrium partitioning when applicable, the second stage difficient coefficients were estimated.

Equilibrium partitioning of BHT between the copolymers and solvents was observed only for the case of E-5\% VA and water at $60^{\circ} \mathrm{C}$. In all other experiments with water, a time lapse of six months was not long enough to establish an equilibrium conditions. 


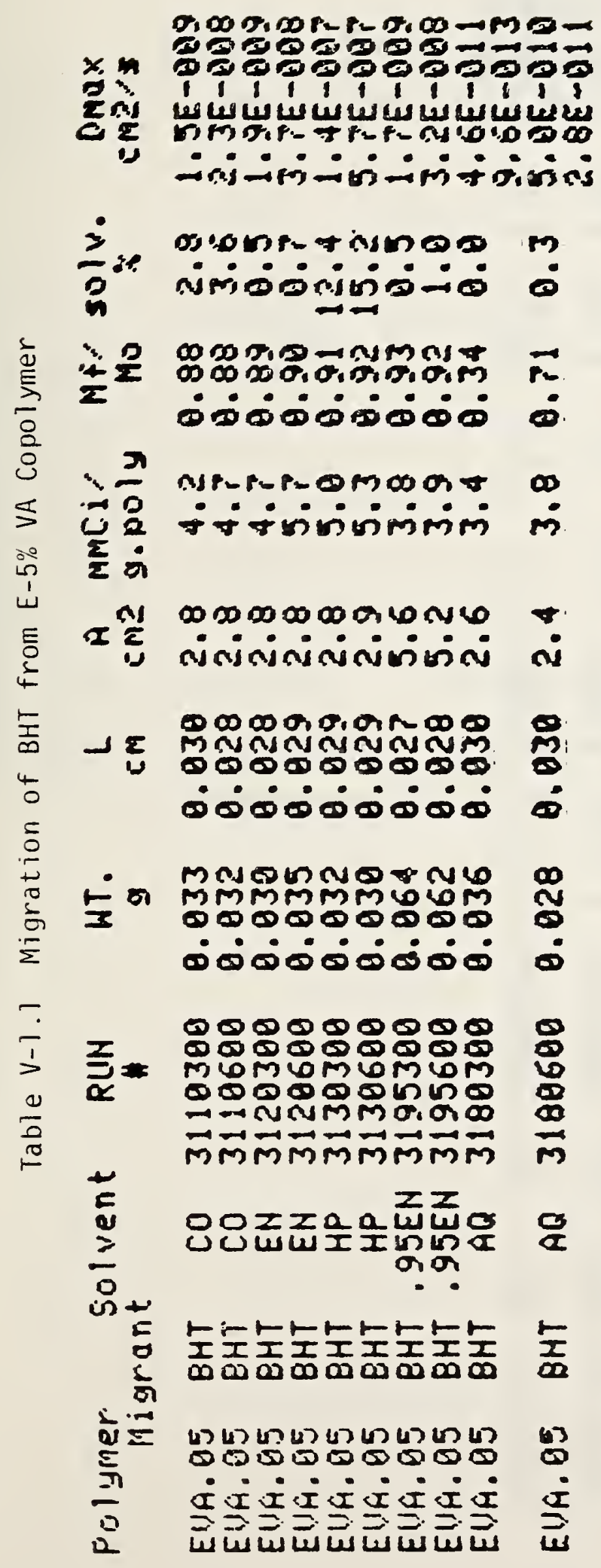




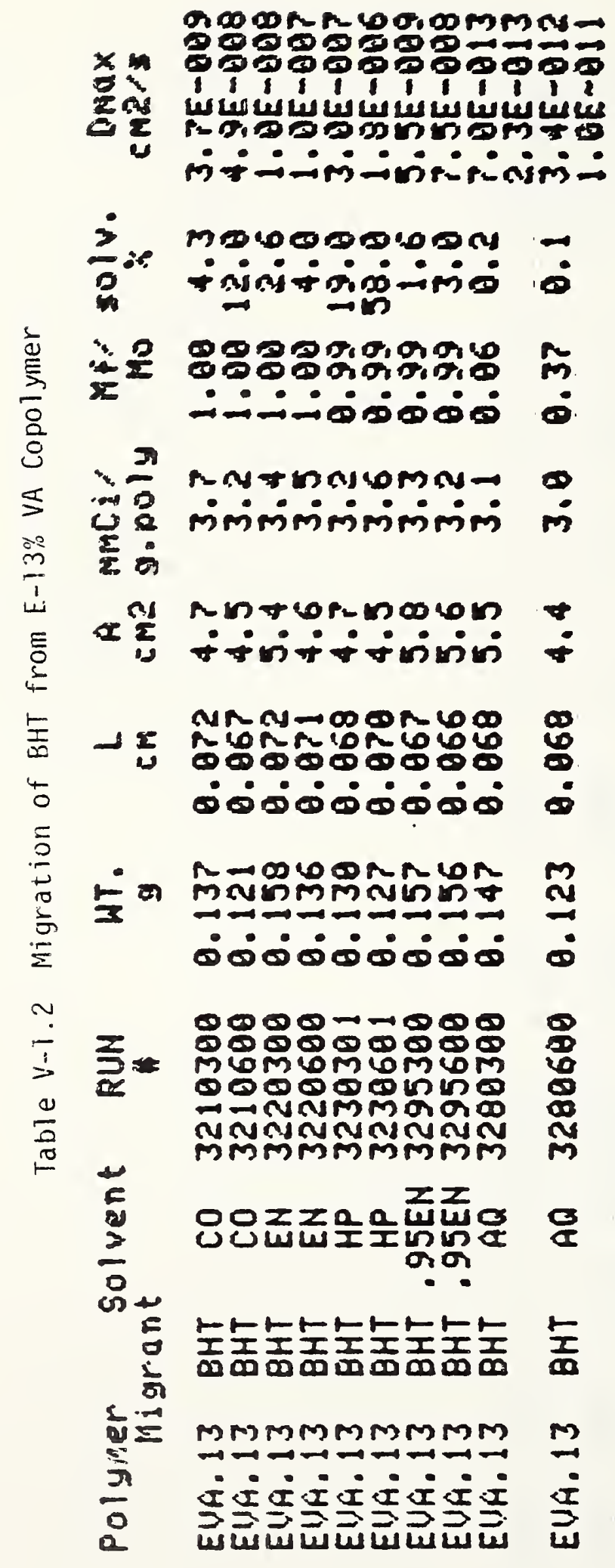


Table $V-2$

Diffusion Coefficients and Activation Energies of

Migration of BHT from Ethylene-Vinyl Acetate Copolymers into Various Solvents

\begin{tabular}{|c|c|c|c|c|c|c|}
\hline Solvent & E5\% V'A & El3\%VA & E5\%VA & El3\%VA & $E 5 \% V A$ & El 3\%VA \\
\hline n-Heptane & 14 & 30 & 57 & 180 & 39 & 51 \\
\hline Corn $0 i 1$ & 0.15 & 0.37 & 2.3 & 4.9 & 76 & 72 \\
\hline Ethanol & 0.19 & 1.0 & 3.7 & 10 & 83 & 65 \\
\hline $95 \%$ Ethanol & 0.19 & 0.55 & 3.2 & 7.5 & 82 & 73 \\
\hline Water - 1st Stage & 0.0046 & 0.00007 & 0.005 & 0.00034 & 67 & 44 \\
\hline 2nd Stage & 0.000096 & 0.000023 & 0.0028 & 0.0010 & 94 & 106 \\
\hline
\end{tabular}


Figure $V-1.1-A, B, C$ Migration of BHT from E-5\% VA Copolymer at $30^{\circ} \mathrm{C}$ Figure $\mathrm{V}-1.2-\mathrm{A}, \mathrm{B}, \mathrm{C}$ Migration of $\mathrm{BHT}$ from E-5\% VA Copolymer at $60^{\circ} \mathrm{C}$ Figure $V-1.3-A, B, C$ Migration of BHT from E-13\% VA Copolymer at $30^{\circ} \mathrm{C}$ Figure V-1.4-A, B, C Migration of BHT from E-13\% VA Copolymer at $60^{\circ} \mathrm{C}$

Legends for Figure $\mathrm{V}-1.1$

$\begin{array}{ll}\text { Corn 0il } & \triangle \\ \text { Ethanol } & \bigcirc \\ 95 \% \text { Ethanol } & 0 \\ \text { n-Heptane } & + \\ \text { Water } & *\end{array}$




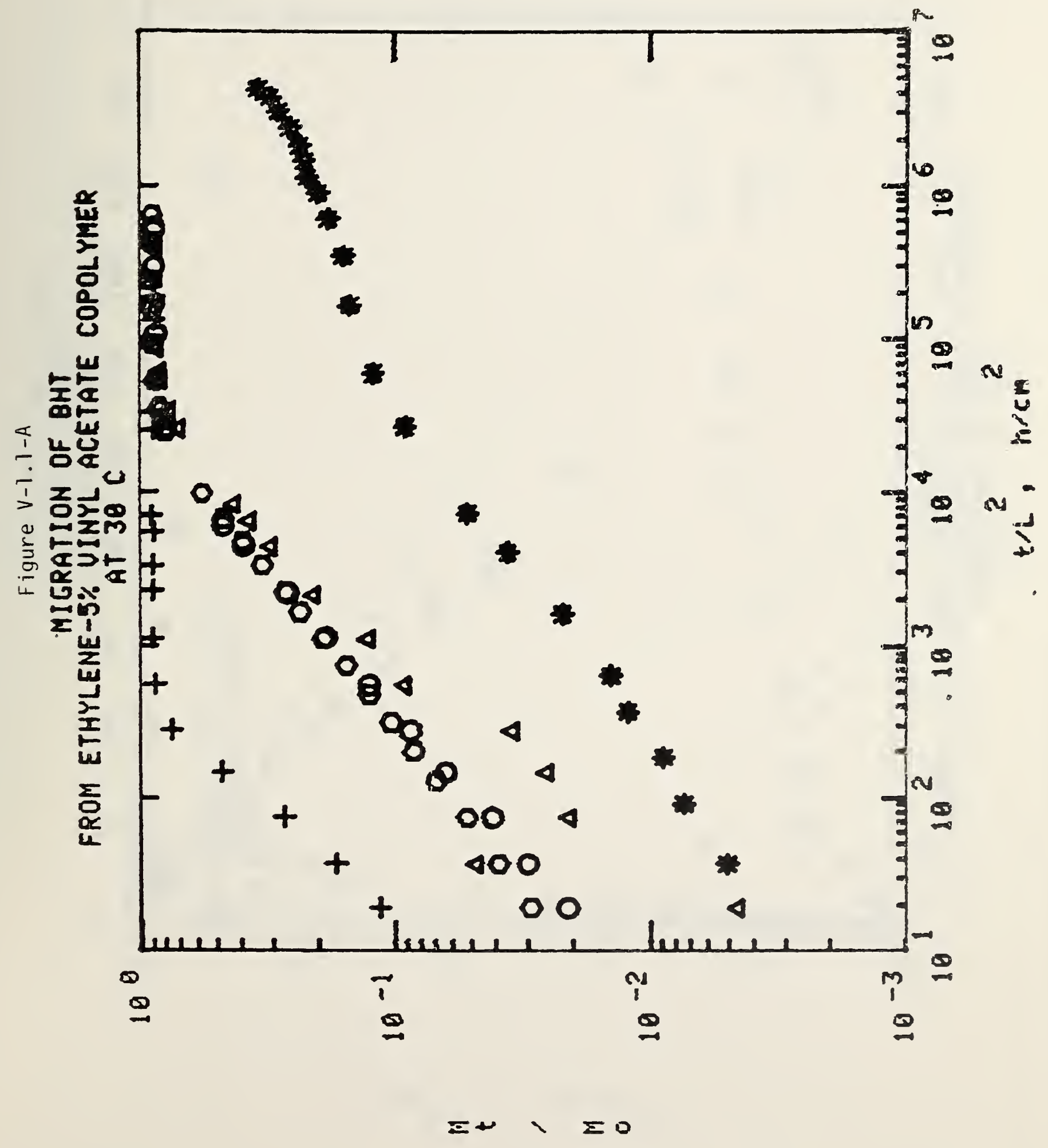




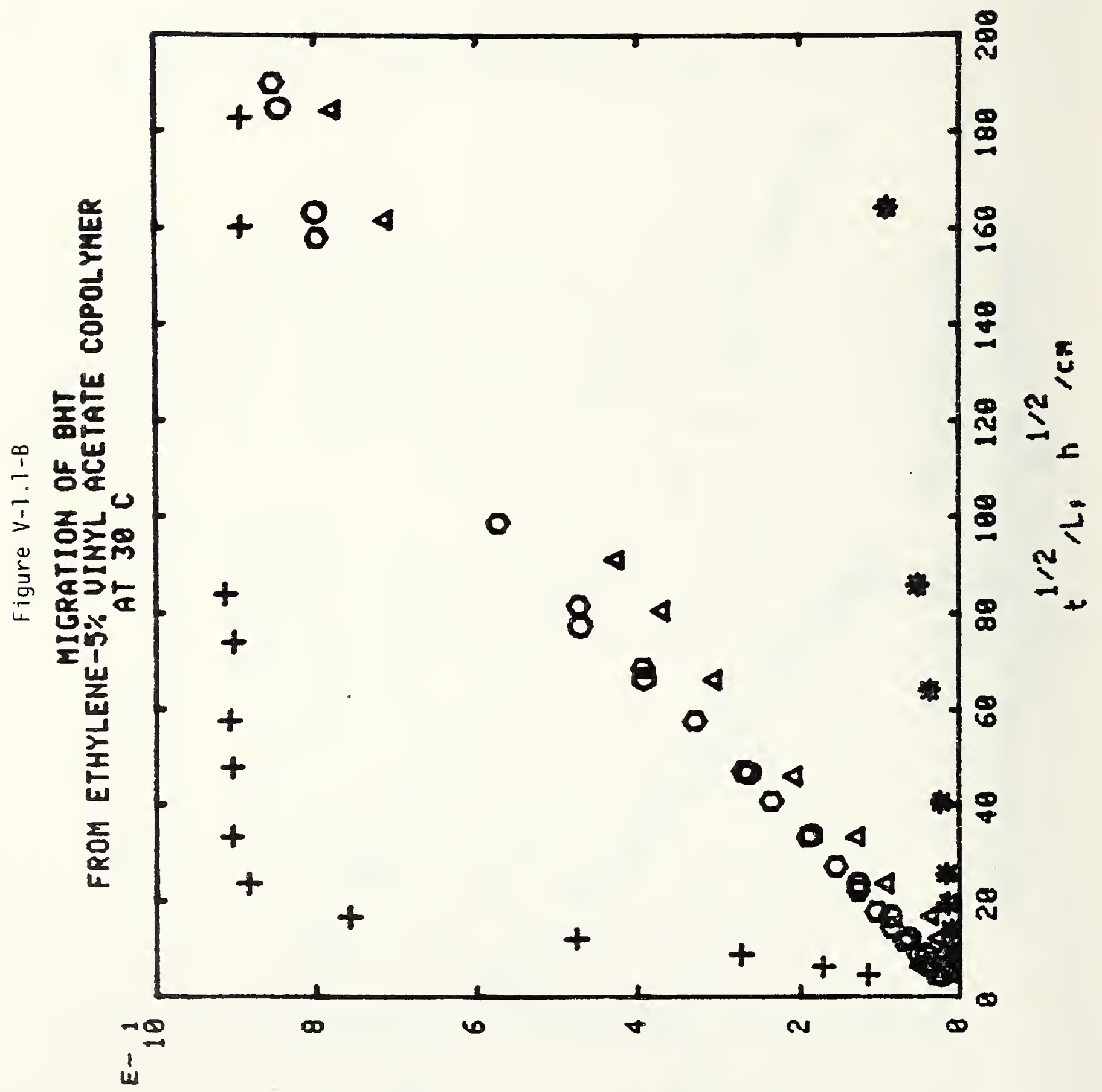

$=+\quad>\quad=0$ 


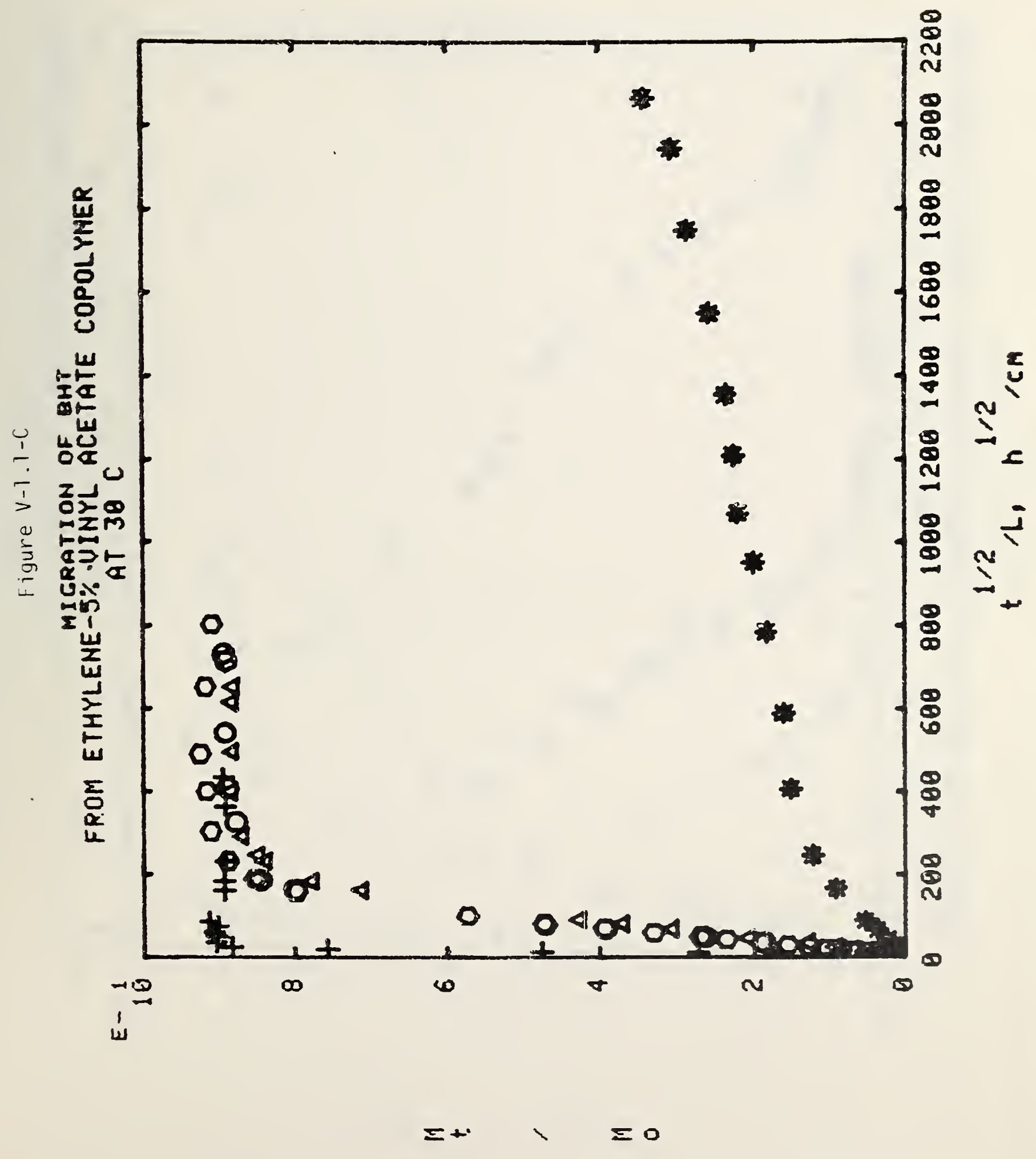




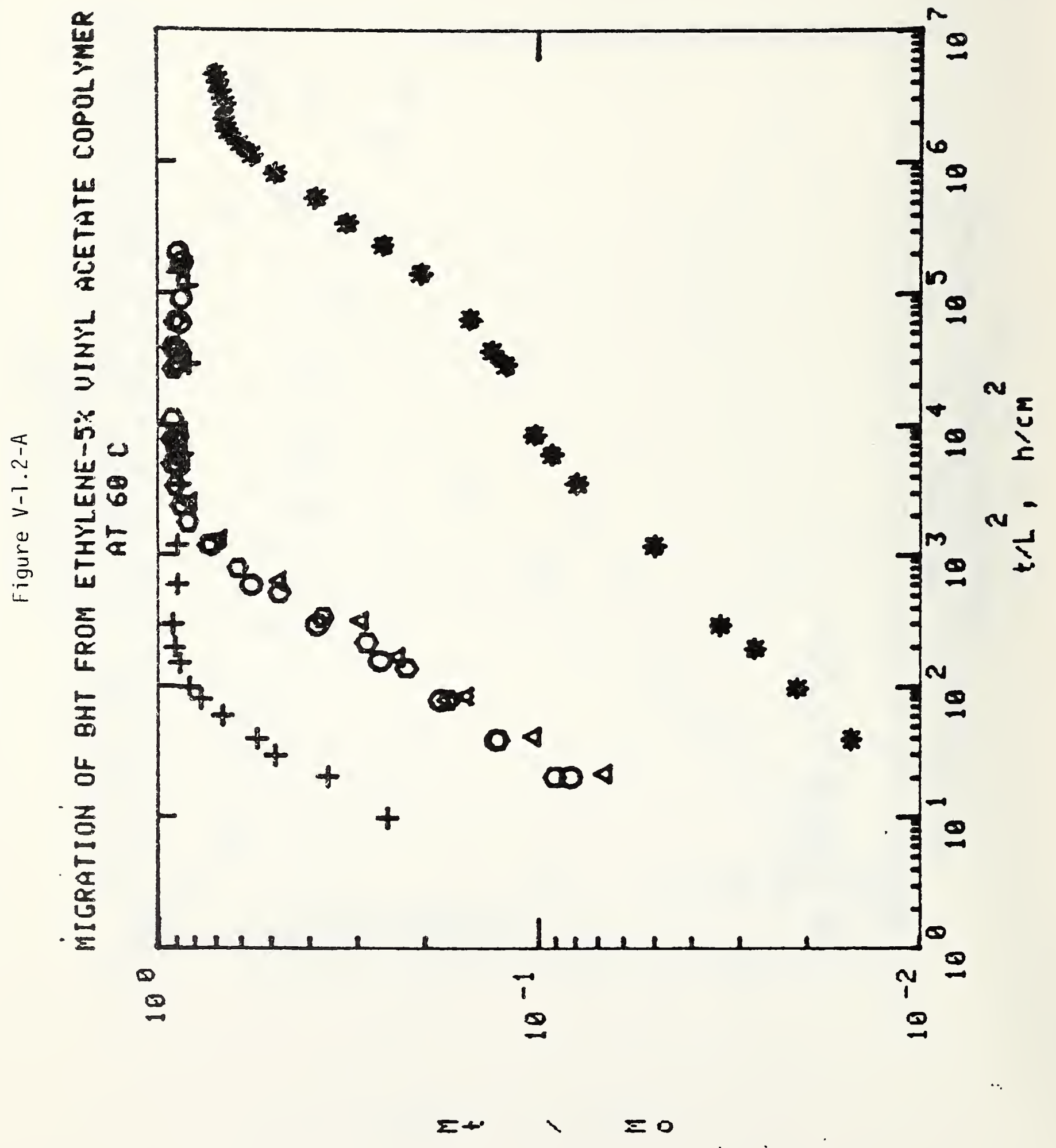




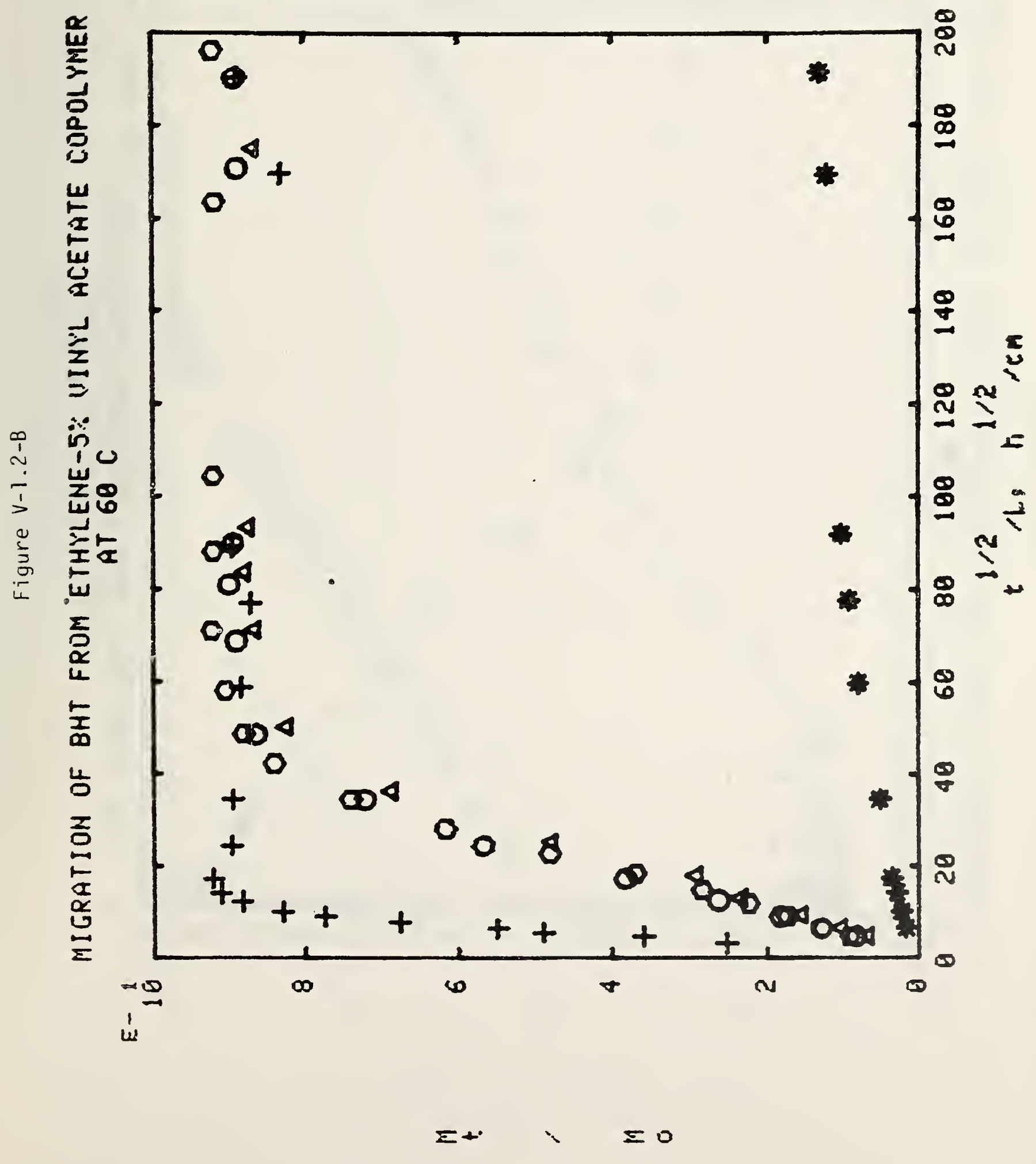




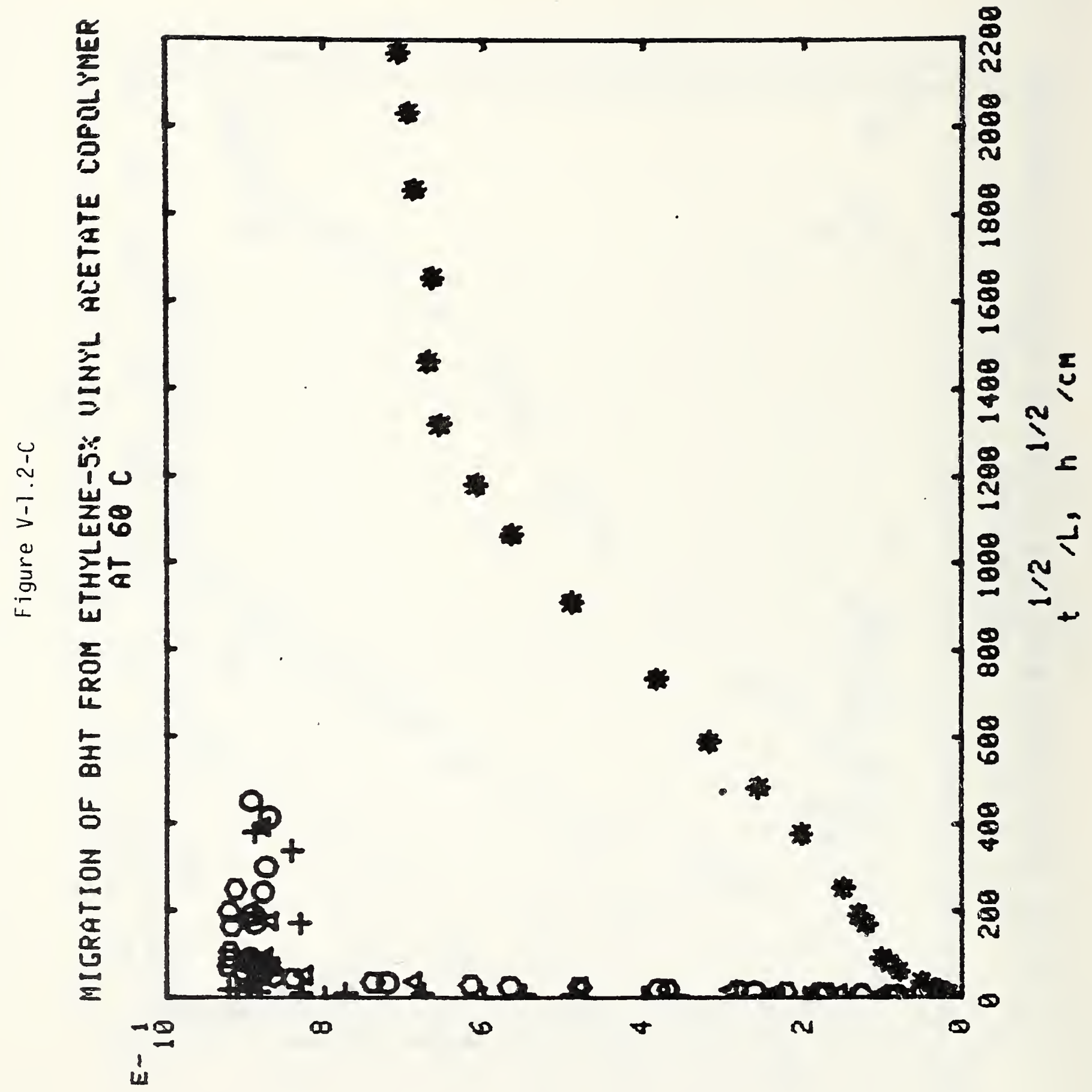

$\sum+\quad y \quad \sum 0$ 


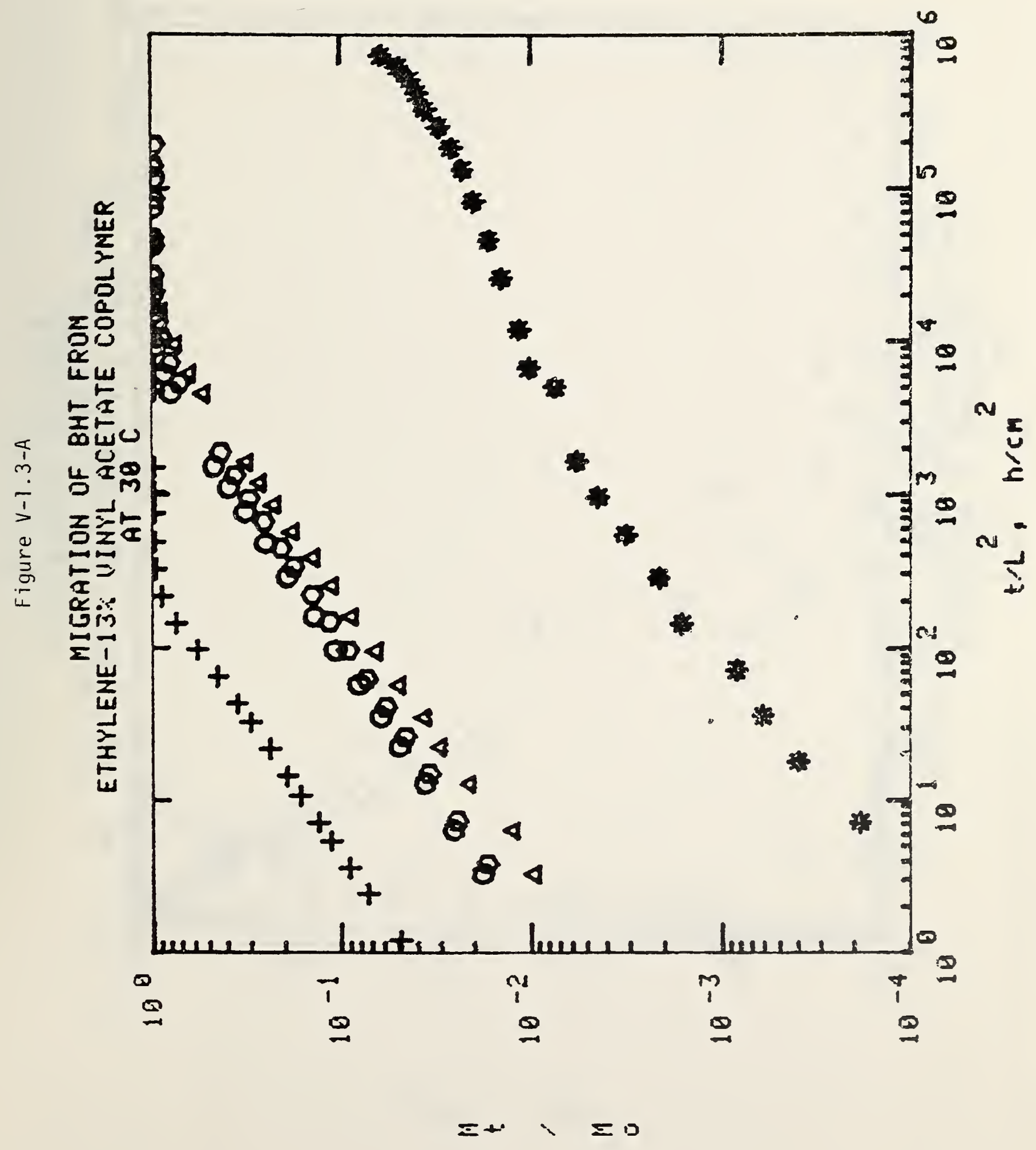




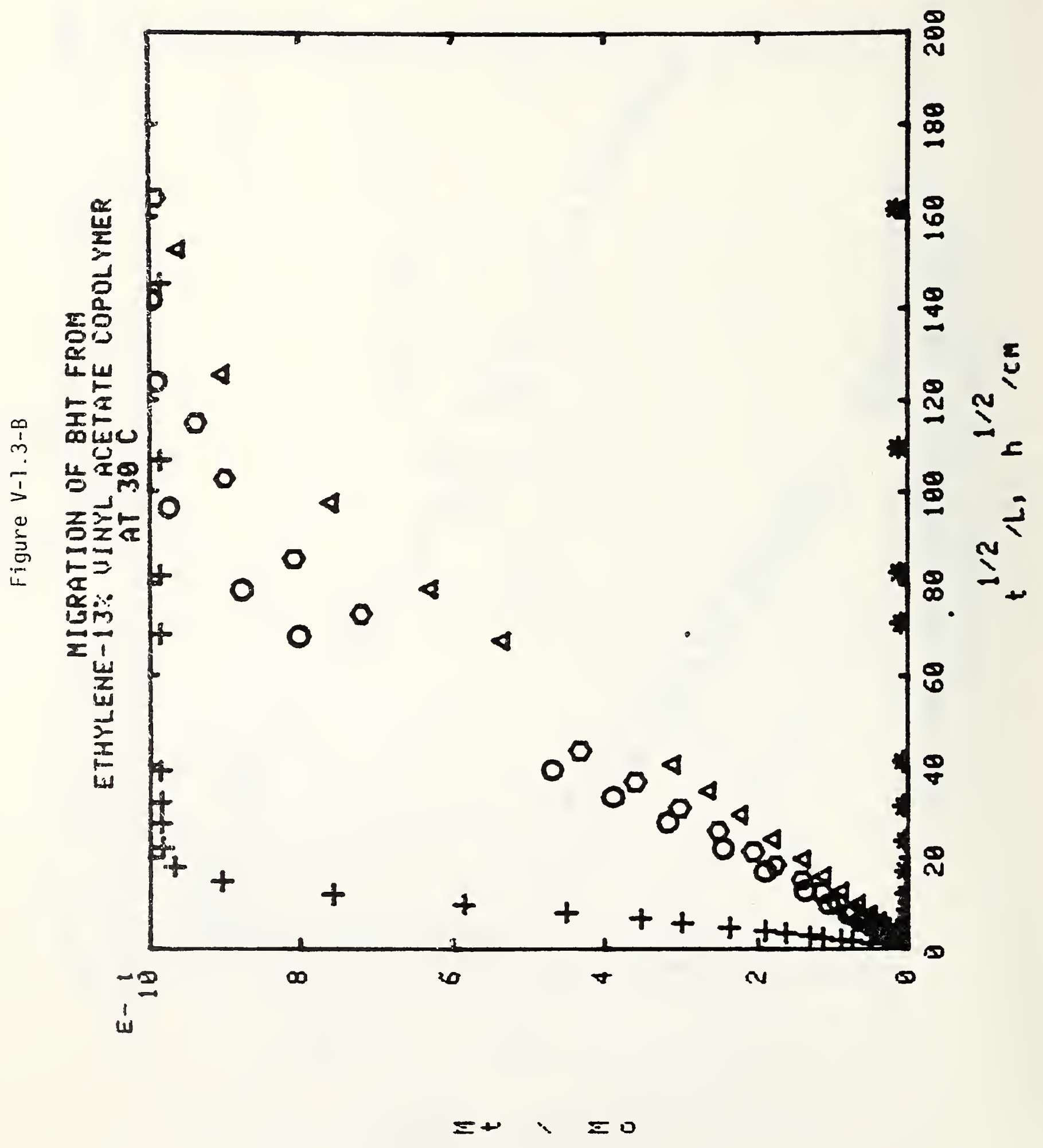




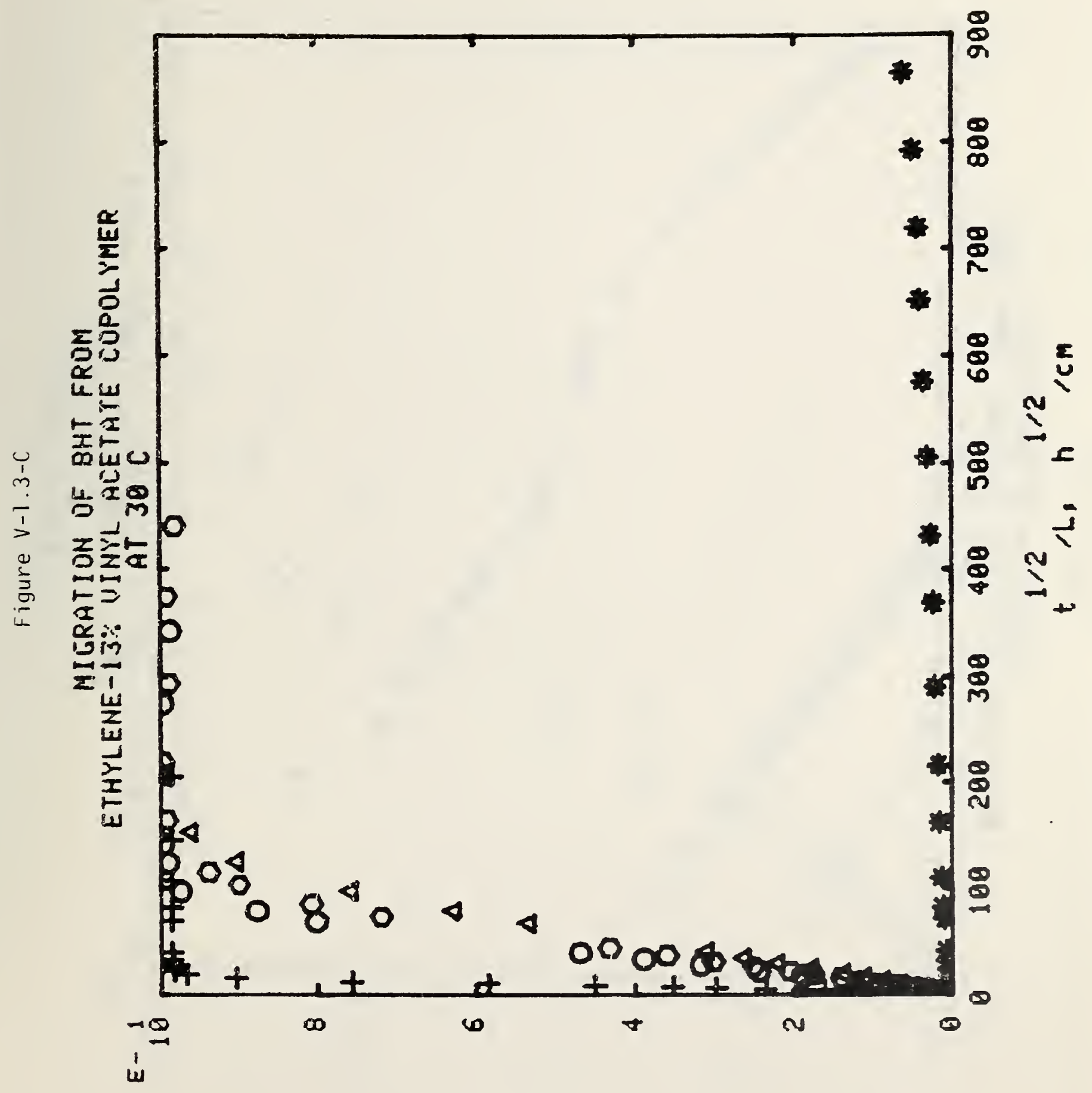

$\sum+\quad \leq 0$ 


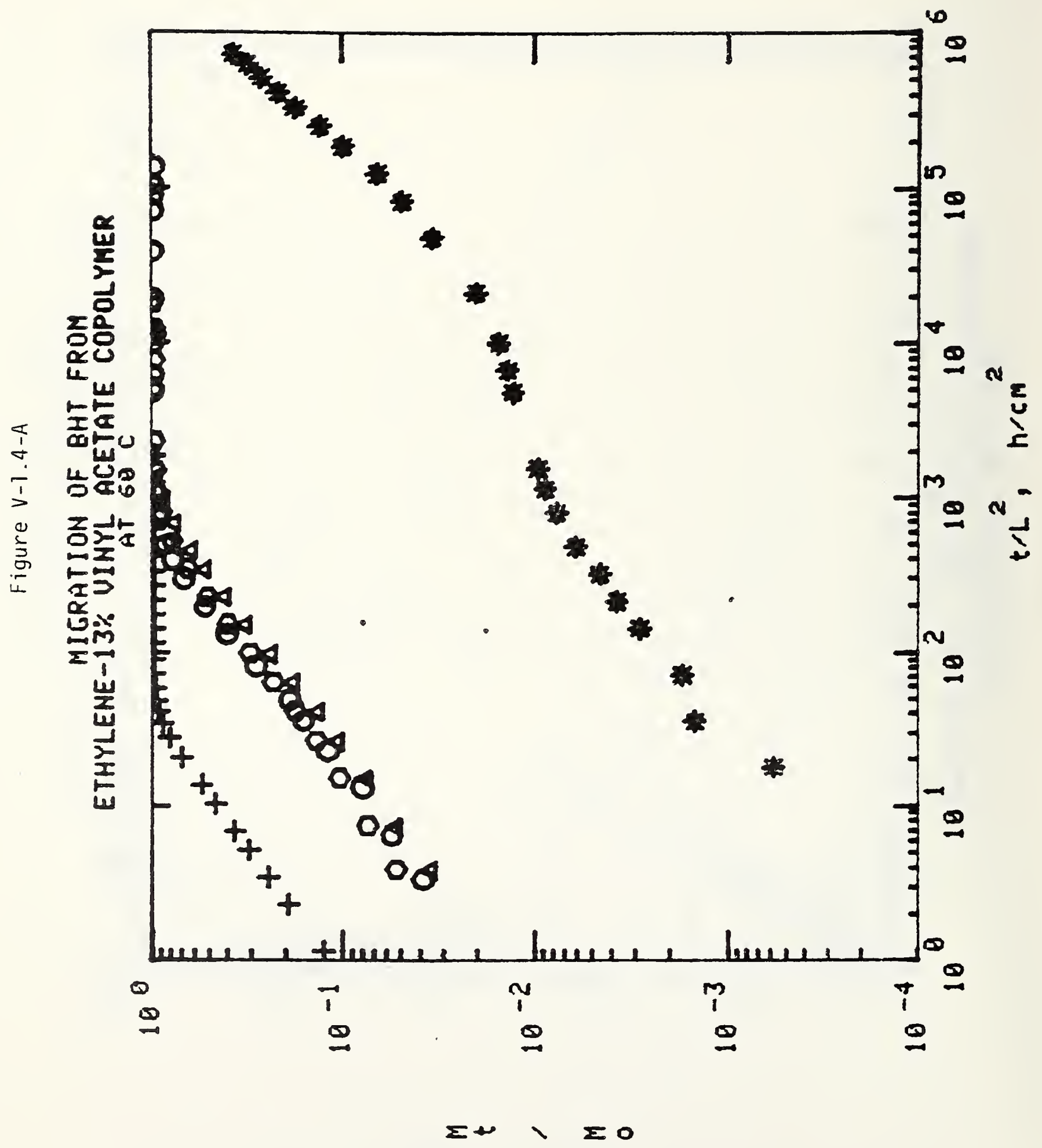




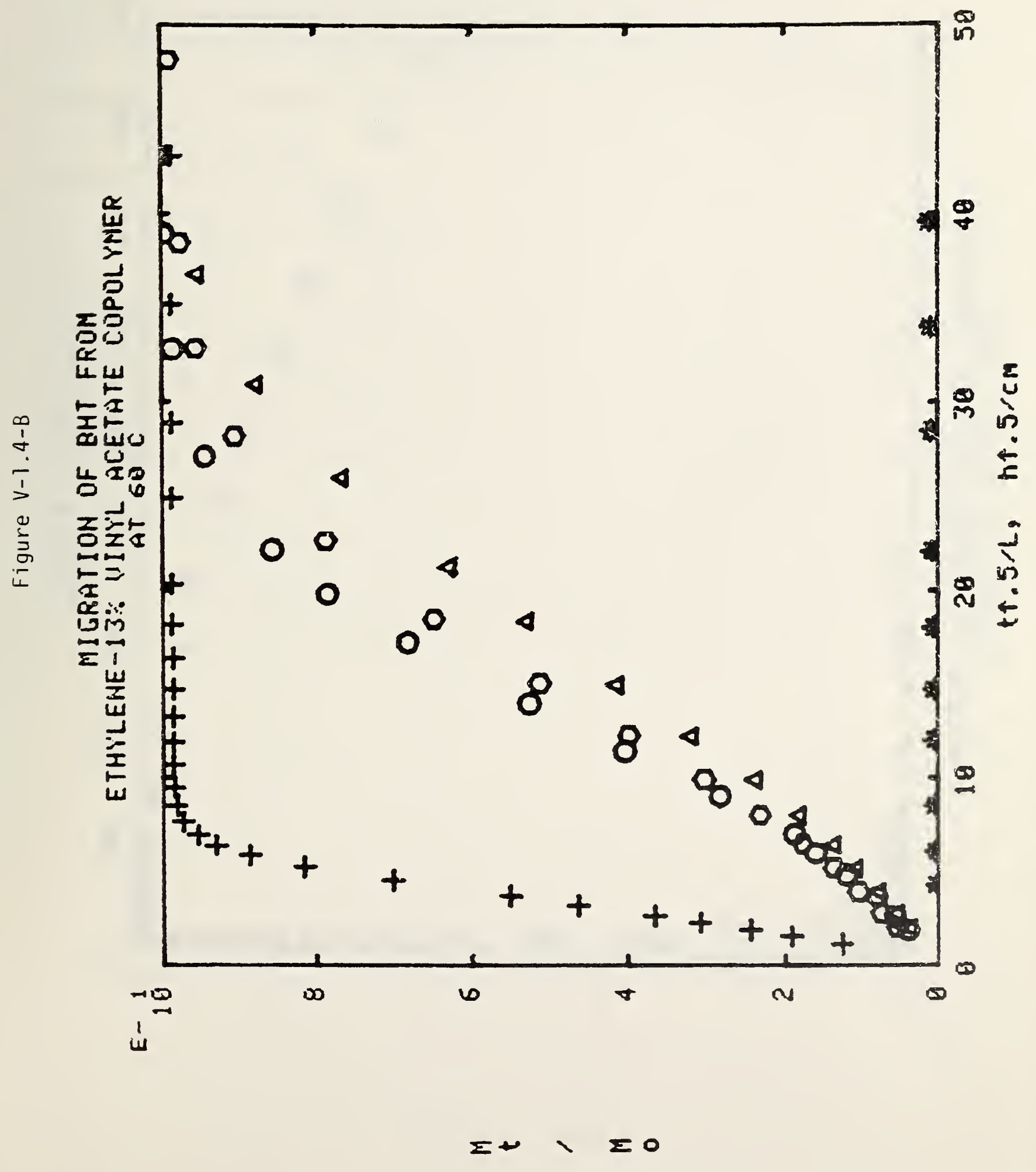




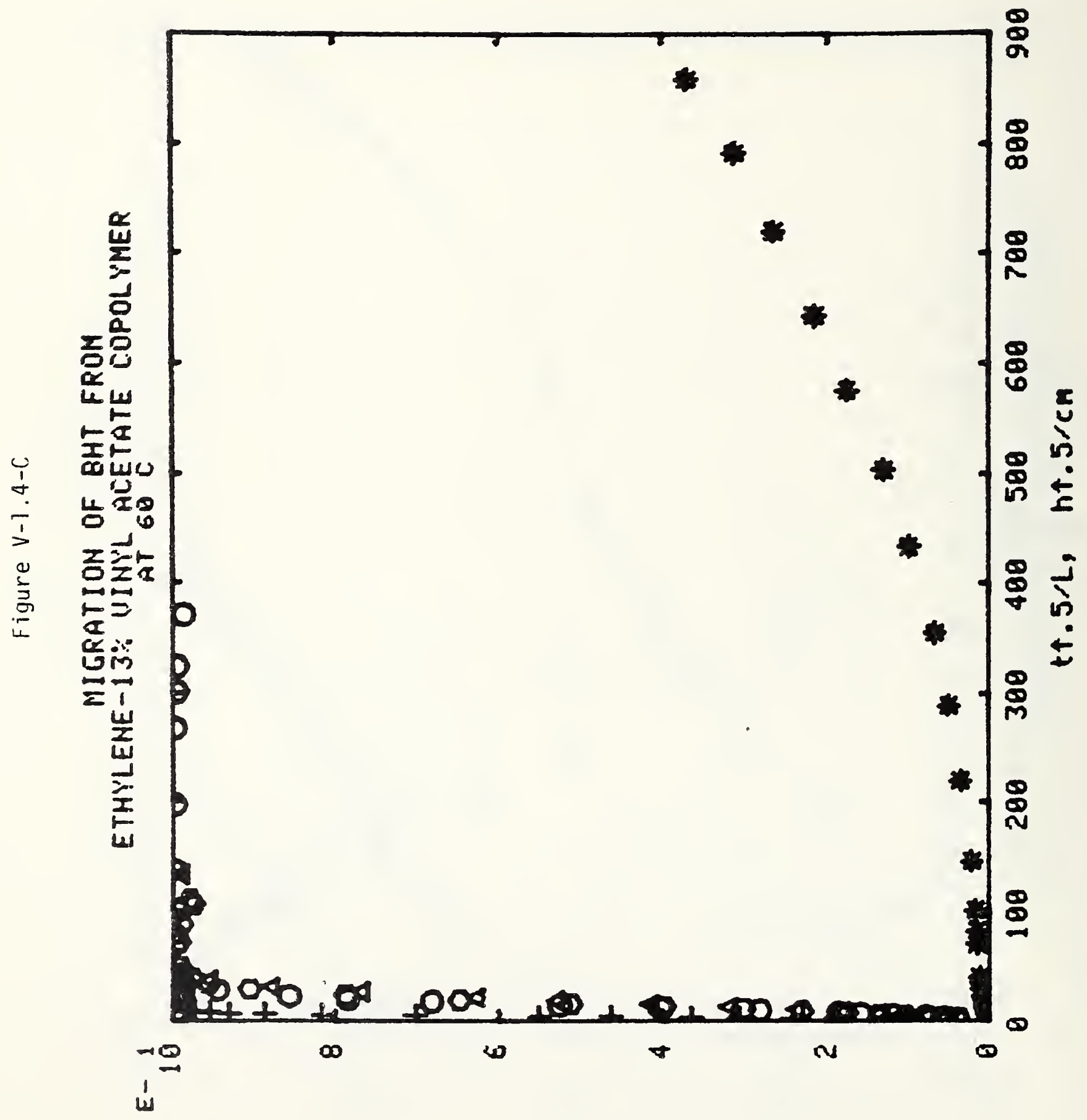

$\Sigma+\backslash \geq 0$ 
Figure V-2.1 Migration of BHT from E-VA Copolymers into $n$-Heptane Figure V-2.2 Migration of BHT from E-VA Copolymers into Corn 0i1 Figure V-2.3 Migration of BHT from E-VA Copolymers into Ethanol Figure V-2.4 Migration of BHT from E-VA Copolymers into $95 \%$ Ethanol Legends for Figure $V-2$

$\begin{array}{ccc} & E-5 \% \text { VA } & E-13 \% \text { VA } \\ 30^{\circ} \mathrm{C} & + & \diamond \\ 60^{\circ} \mathrm{C} & x & \square\end{array}$




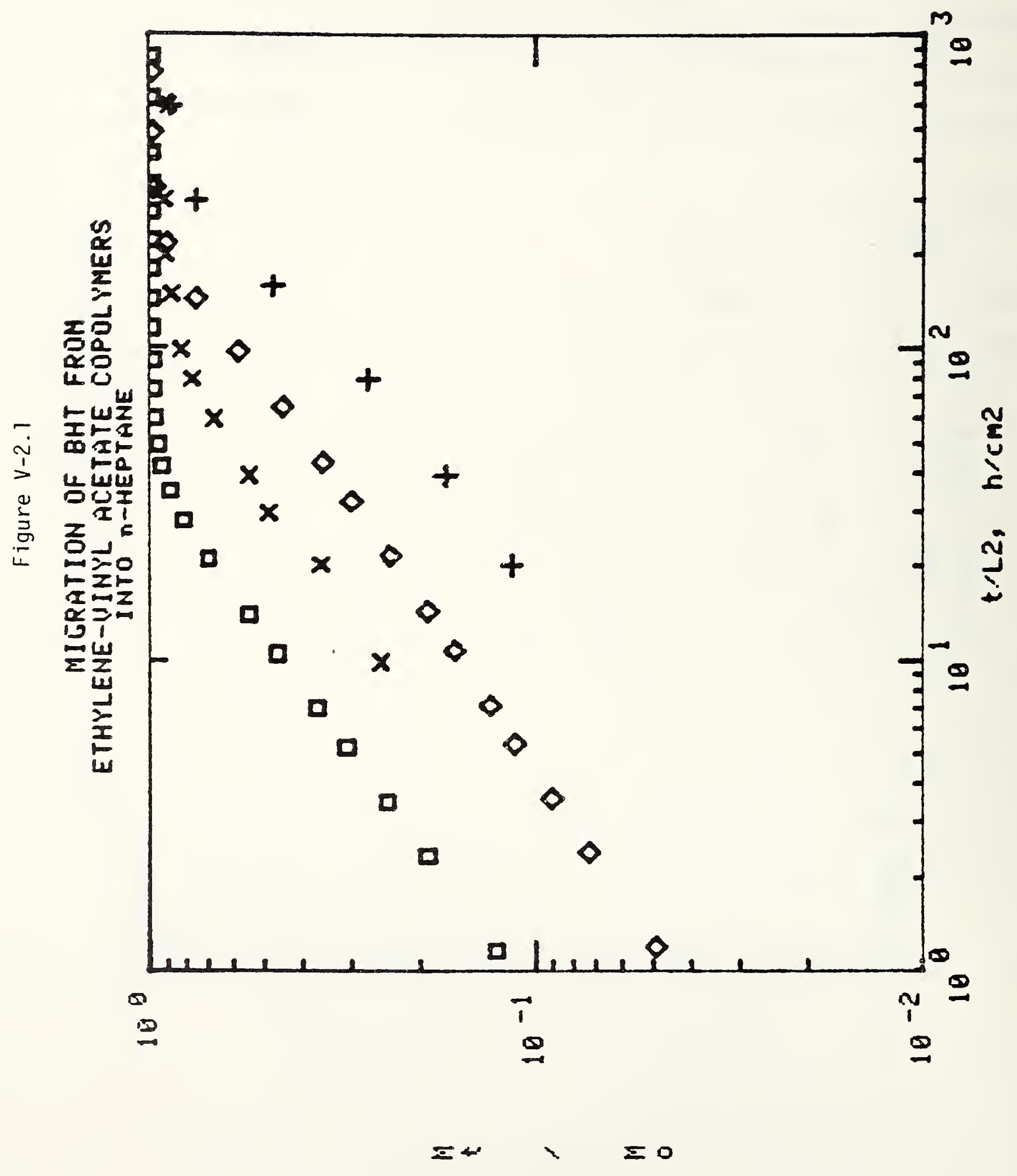




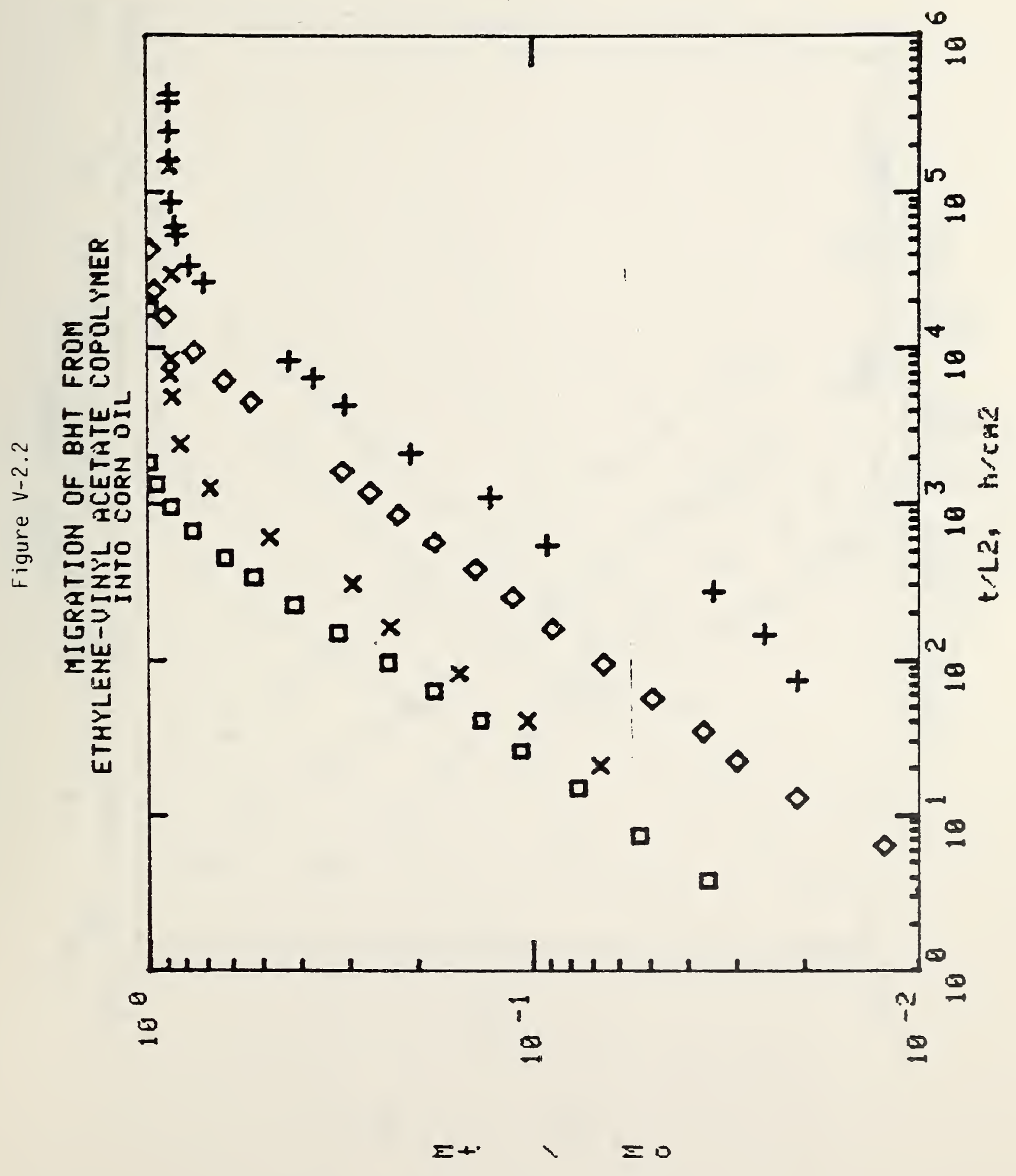




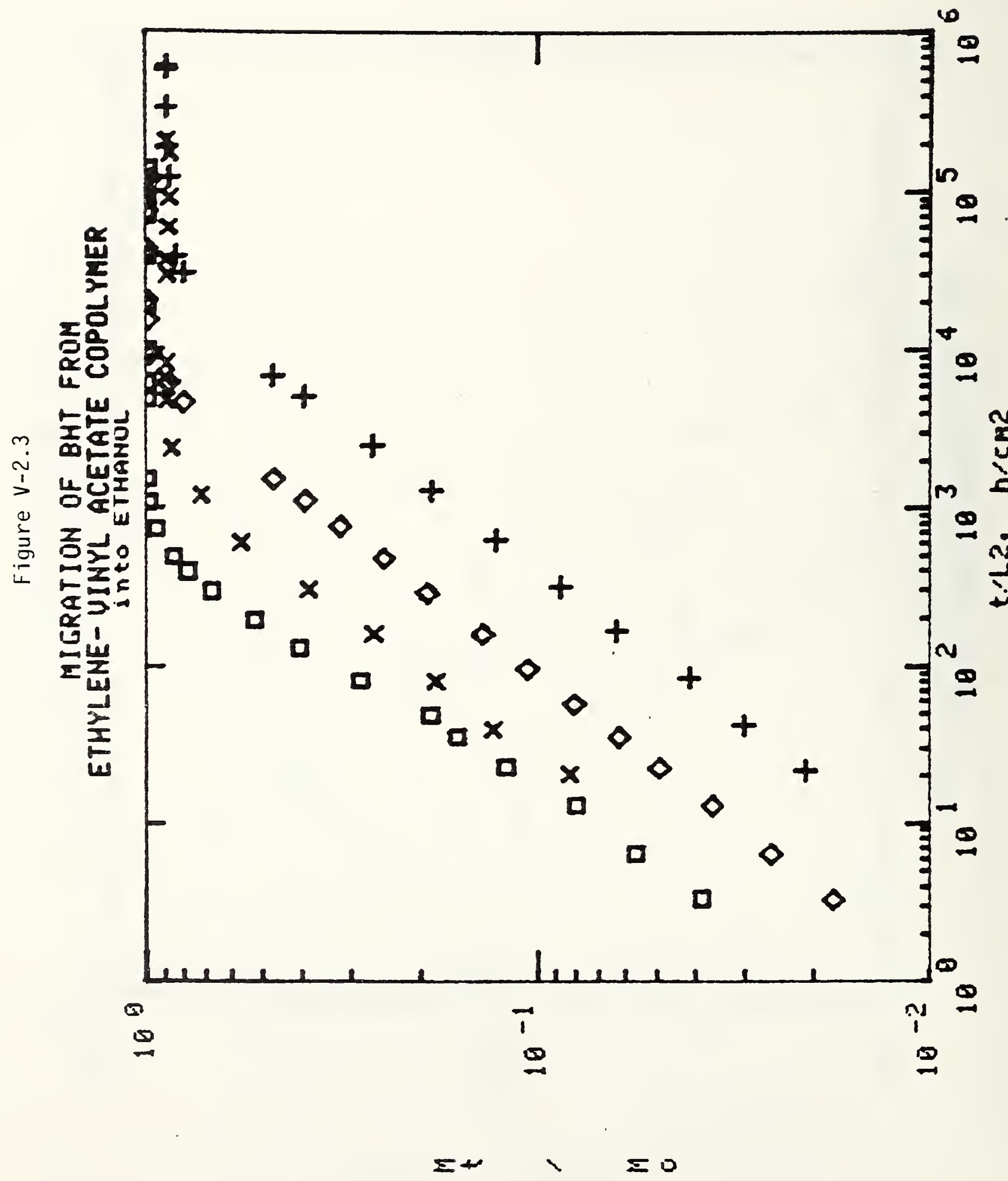




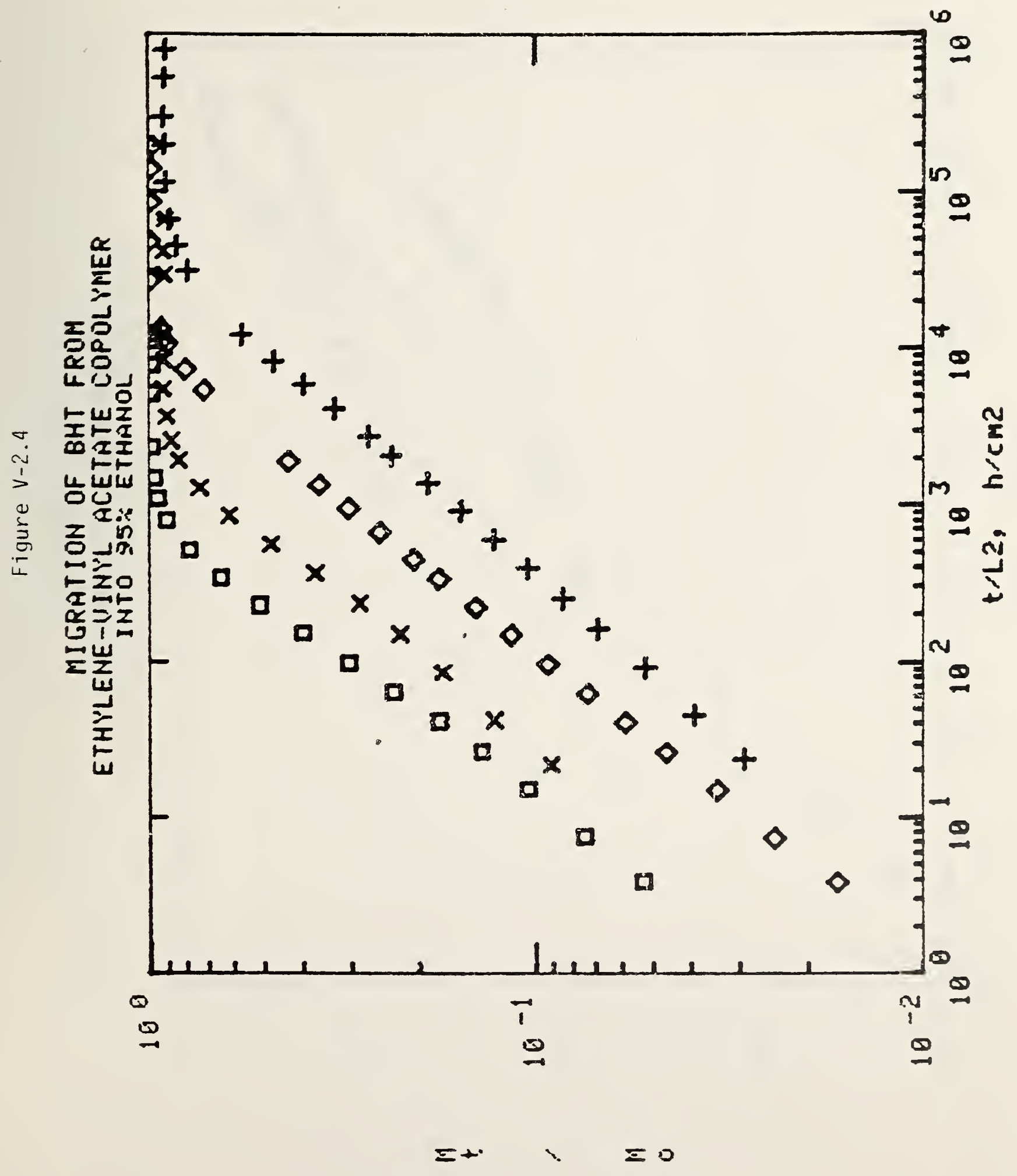


Figure $V-3 A, B, C$ Migration of BHT from E-VA Copolymers into Water

\section{Legends}

$\begin{array}{ccr} & E-5 \% \text { VA } & E-13 \% \text { VA } \\ 30^{\circ} \mathrm{C} & + & \bigcirc \\ 60^{\circ} \mathrm{C} & \times & \square\end{array}$




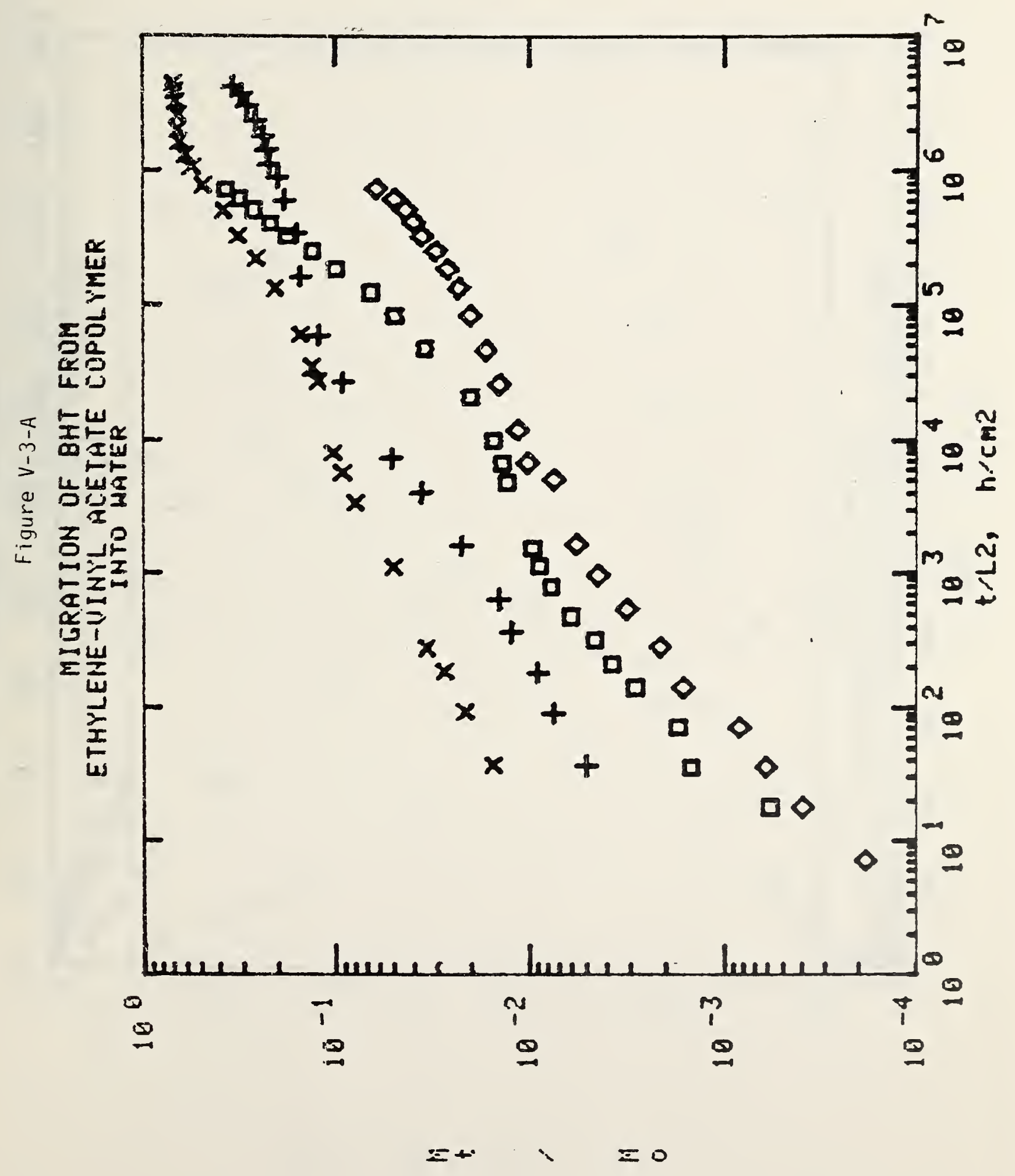




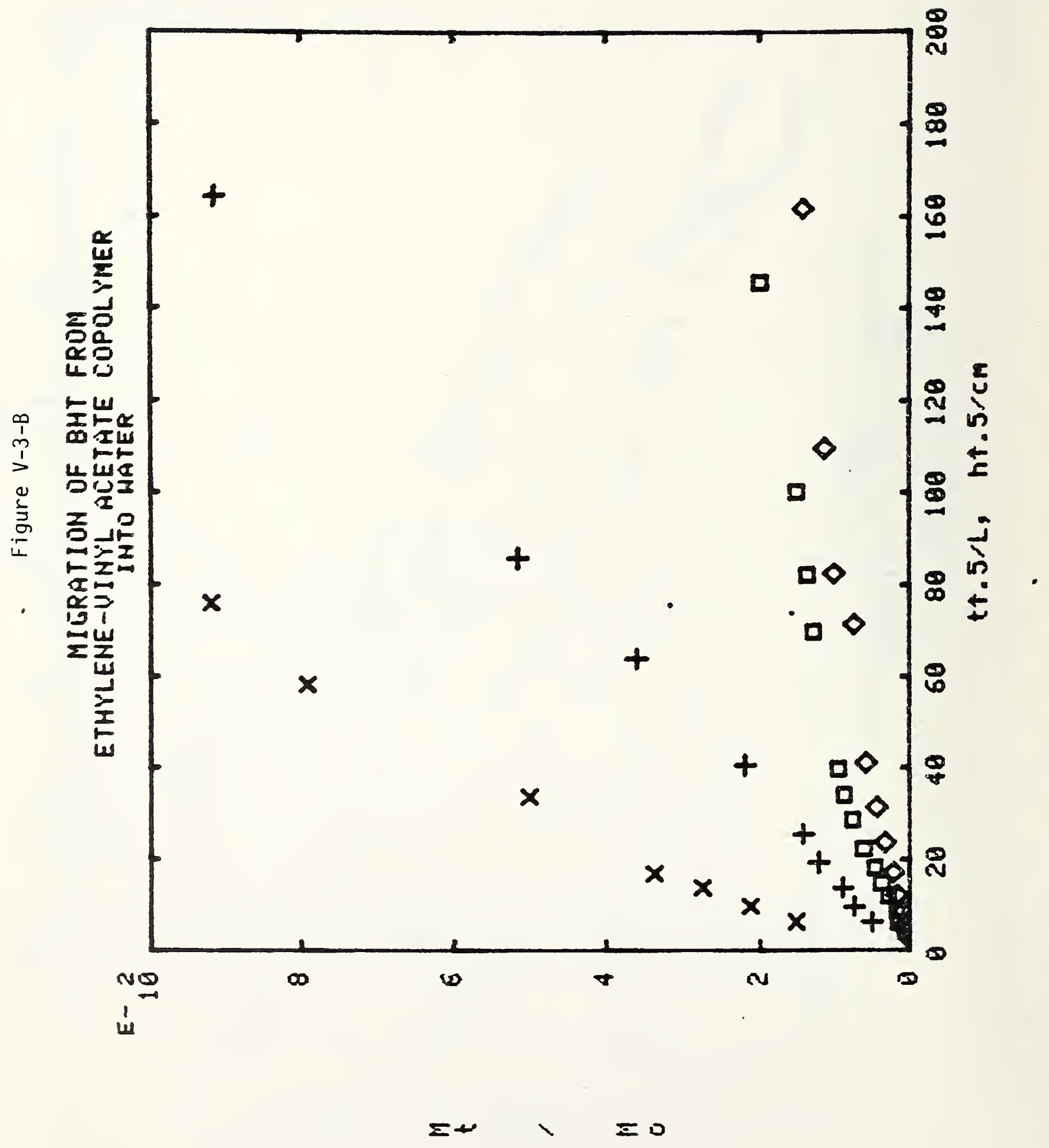




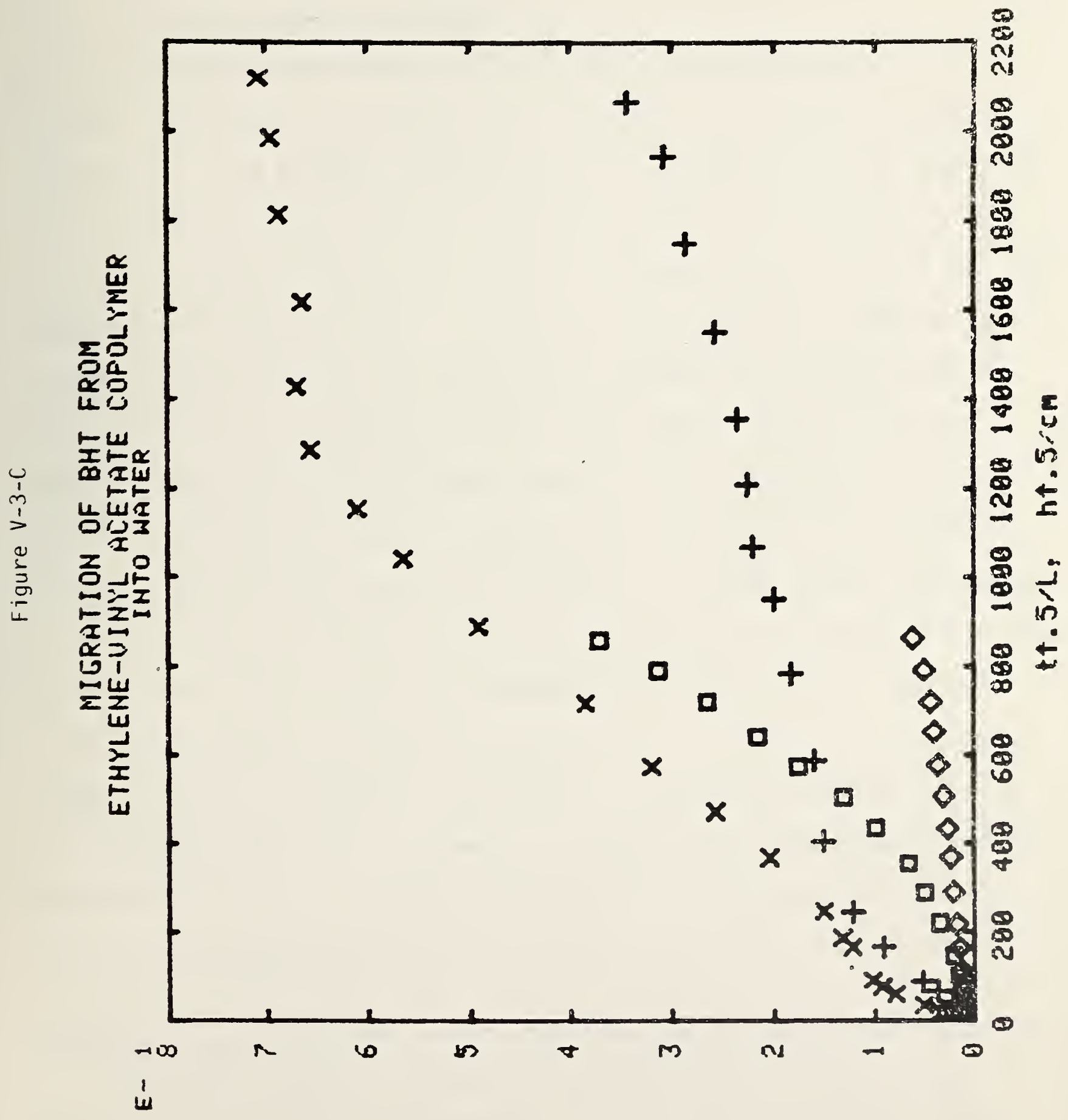

$\pm+\quad \backslash \quad=0$ 

VI. IGC Determinations of the Relative Diffusion Coefficients of Several Probes in Linear Polyethylene

Inverse gas chromatography (IGC) studies on the determination of diffusion constants in polyothylene were reported in the previous annual report [1]. These findings indicate that while accurate absolute diffusivity measurements seem beyond the reach of the IGC method, the possibility exists for using the technique to rank the relative ease of diffusion of many probes in a given polymer. A calibration of a specific column can be accomplished by conducting experiments with a probe-polymer combination of known diffusivity to calculate the mean polymer layer thickness from the measured peak broadening. The same column can then be used under similar operating conditions to determine other probe diffusivities and a relative ranking of many probe diffusion coefficients can be established. Experiments on several seriegs of compounds have been conducted and those for which the data analysis and reduction have been completed will be used to illustrate the procedure of relative diffusivity determinations. Preceding this discussion, however, will be: a review of the theory for determining absolute diffusion constants from IGC studies, a summary of results from polyethylene-octadecane experiments to be used in the relative diffusivity determinations, and a section giving experimental details on the relative diffusivity measurements.

\section{A Review of Pertinent Theory for IGC Absolute Diffusivity Determinations}

The probe or migrant injected onto a chromatographic column resides partly in the carrier gas and partialiy in the polymer or stationary phase during transit through the columr. Diffusion of the probe vapor in the gas phase can occur parallel to the background flow of carrier gas sweeping the vapor through the column both in the direction of and opposed to the carrier flow. This longitudinal or axial gaseous diffusion process causes an increase 
in width of the eluted peak over its initial injection distribution. An additional gas phase peak spreading mechanism originates in the multiplicity of path lengths possible around the packed support particles and leads to an associated broadened distribution of elution times. Peak spreading can also be attributed to diffusive processes in the stationary phase. If the carrier gas flow rate is very rapid, steady-state partitioning of the vapor between the gas and stationary phases cannot be established. The residence time in the stationary polymer phase is then influenced by both the diffusivity of the probe in the polymer and the distance in the stationary phase over which diffusion must occur, among other factors. The peak broadening processes discussed above are commonly quantified by the convenient construct of the height equivalent to a theoretical plate, $H$, which is related to the width at half-height, $w_{1 / 2}$, for an eluted Gaussian-shaped peak as follows:

$$
H=\frac{L}{8 \ln (2)}\left(\frac{w_{1 / 2}}{t_{R}}\right)^{2}
$$

where $L$ is the column length, and $t_{R}$ the retention time of the peak at the maximum. The determination of these quantities from a Gaussian-shaped experimental chromatogram with a peak standard deviation of $\sigma$ is illustrated in the schematic of Figure VI-I.

An equation attributed to van Deemter et al. [2] is often used to relate $H$ to the local carrier gas flow velocity, $u$, and is of the form:

$$
H=A+B / U+C u
$$

The A term arises from the number of possible gas paths through the column; $B$ is from longitudinal diffusion of the probe in the carrier gas; and $C$ originates from the resistance to mass transfer in the polymer or stationary 
phase. The coefficients in equation 2 are defined as follows:

$$
\begin{aligned}
& A=4 \lambda r \\
& B=2 \gamma D_{g} \\
& C=\frac{8 s^{2} k}{\pi^{2} D_{s}(1+k)^{2}}
\end{aligned}
$$

where $\lambda$ is a measure of support packing irregularities, $r$ the average support particle radius, $\gamma$ a correction coefficient for constriction and tortuosity of the gas flow path in the column, $D_{g}$ the probe diffusivity in the gas phase, $s$ the effective thickness of the stationary phase film, $D_{s}$ the diffusivity of the probe in the stationary phase, and $k$ the column capacity factor commonly given as:

$$
k=\frac{t_{R}-t_{M}}{t_{M}}
$$

where $t_{M}$ is the time taken to elute an unsorbed marker material from the column. Equation 2 indicates that, at large values of the flow rate, $H$ will be proportional to the flow rate. The probe-polymer diffusion coefficient can then be determined from the constant of proportionality $C$ by equation 5 if the effective film thickness is known. Equation 5 is not applicable for collimns packed with glass bead support particles. In such cases of diffusion into a uniform stationary phase $\mathrm{film}$ on a solid surface, a similar relation for $\mathrm{C}$ applies:

$$
C=\frac{2 s^{2} k}{3 D_{s}(1+k)^{2}}
$$


While equation 2 gives a suitable explanation of peak spreading in some cases, other results have raised questions about its utility, particularly when significant gas phase diffusion can occur. These discrepancies indicate that the simplifications made by van Deemter in neglecting other mechanisms of diffusion are not valid for all systems. Giddings and coworkers [3] examined several modified forms of the van Deemter equation and found that none could account for the experimental results obtained on columns of varying length and outlet pressure.

An equation proposed by Jones [4] takes several additional sources of peak spreading into consideration: resistance to mass transfer in the gas phase normal to the carrier gas flow direction (D), carrier gas velocity distribution effects $(E)$, and a correlation between these two processes $(F)$. This equation is given as:

$$
H=A+B / U+(C+D+E+F) u
$$

where $C$ is as in equation 7 and

$$
\begin{aligned}
& D=\frac{C_{D} k^{2} g^{2}}{D_{g}(1+k)^{2}} \\
& E=\frac{4 C_{E} r^{2}}{D_{g}} \\
& F=\frac{4 \theta\left(C_{D} C_{E}\right)^{1 / 2} k r g}{D_{g}(1+k)}
\end{aligned}
$$

$g$ being the diffusion path length in the gas, $\theta$ the correlation coefficient between the $D$ and $E$ terms, and $C_{D}$ and $C_{E}$ geometric constants. 
The radial diffusion 0 term was discounted by van Deemter and co-workers in the derivation of equation 2 because of the much greater magnitude of $\mathrm{D}_{\mathrm{g}}$ compared to $D_{S}$. This simplification is valid for the thick liquid films used in their work, studies of columns with roughly 0.3 mass fraction stationary phase loading. The diffusion distances 5 and $g$ are roughly comparable under these circumstances. If low column loadings which reduce s to about $0.01 \mathrm{~g}$ are used, then the much larger probe diffusivity in the gas phase is overcome by the ease of mass transfer through the thinly-spread liquid phase and the $D$ term cannot be ignored.

The E contribution to spreading arises from diffusion caused by differences in the velocity of the carrier gas in various parts of the column. Velocity differences can arise from both short and longer range channels formed by support packing irregularities and from trans-column effects caused by the influence of the tube walls. These effects are summarized by Giddings [5] who estimates approximate magnitudes for contributions of the many possible velocity gradients combined in the $E$ term cf equation 8.

While the first three terms in equation 8 are independent of one another, the $D$ and $E$ terms are not since molecules which have a lower average velocity will spend a smaller fraction of time in the gas phase and therefore not experience as great a percentage of gaseous diffusion as molecules with higher than average velocity. The total variance, $\sigma_{i j}^{2}$, of two such dependent contributors, $\sigma_{i}^{2}$ and $\sigma_{j}^{2}$, is given as:

$$
\sigma_{i j}^{2}=\sigma_{i}^{2}+\sigma_{j}^{2}+2 \theta_{i j} \sigma_{i} \sigma_{j}
$$

where the correlation coefficient between the two contributors is denoted as $\theta$. The $F$ term of equation 11 originates from the final term in the above relation and corresponds to the interaction between the $D$ and $E$ terms. 
Giddings [6] has given an expression said to account for all known plate height terms contributing at least one percent of the total plate height for packed GC columns in the absence of interfacial adsorption as:

$$
H=\sum_{i} \frac{1}{1 / A_{i}+1 / E_{i} u}+\frac{B}{u}+\sum_{i} C_{i} u+D u+G
$$

The $G$ term represents the contribution of column-wide velocity inequality effects, such as that arising from column coiling, and could alternatively be included in $E$. This equation is otherwise similar to equation 8 except for its omission of the interaction term $F$. A compilation of the $C$, D, and E coefficients expected to apply in various situations and a means of calculating their magnitude from measurable column parameters is given in reference [5].

The plate height equations discussed up to this point apply only to the peak spreading in a short region of the chromatographic column where the carrier velocity $u$ is a constant local to that region. A final modification can be made to equation 8 or 13 to account for the variation in local carrier velocity along the column caused by the drop in pressure from column inlet to outlet. The effect of this correction is primarily on the liquid mass transfer term, as discussed by Dal Nogare and Juvet [7]. The final expression for the dependence of the experimentally accessible column average plate height, $\bar{H}$, on the carrier gas outlet velocity, $u_{0}$, is similar to equation 8 and is given as:

$$
\bar{H}=A+\frac{B}{u_{0}}+\left(\frac{2 p_{0} C}{p_{i}+p_{0}}+D+E+F+G\right) u_{0}
$$


where $p_{i}$ and $p_{0}$ are the column inlet and outlet pressure. The gas phase diffusivity found in equations $4,9,10$, and 11 must also be restated at the outlet pressure of the column. The form of this modification is based on the relationship between the local column velocity and the average carrier gas velocity. The preceeding treatment assumes a distance average of this velocity. Other methods [3] involve the use of a pressure average velocity and yield a slightly different equation for $\bar{H}$ but agree reasonably well with equation 14 even at large values of the inlet pressure.

The magnitudes of coefficients in equation 14 and the configuration of the polymer film on the surface of the supporting beads can have a significant influence on the methods used in interpreting data derived from IGC determinations of diffusive peak broadening as discussed in the previous annual report. These effects will be reviewed briefly for the experimental determination of the diffusivity of normal octadecane in high density polyethylene considered there.

\section{Summary of Previous Results for LPE-Octadecane}

As discussed earlier, IGC determinations of diffusivity in the polymer phase are made from a study of probe peak broadening at varying carrier gas velocities; a schematic illustration of the results is shown in Figure VI- 2 . The local plate height and carrier velocity of equation 2 are commonly replaced by the measured average quantities in experimental determinations of column efficiency. Such $\bar{H}$ vs $\bar{u}$ plots for eight flow velocities in the flow rate region from about 10 to $100 \mathrm{~cm} / \mathrm{s}$ yielded a slope $C$ for column $A$ of $7.05 \mathrm{~ms}$ with calculated standard deviation of 8.8 percent and $18.9 \mathrm{~ms}$ with calculated standard deviation of 7.4 percent for $B$. The van Deemter treatment provides for the calculation of the diffusivity of $c_{18}$ in linear 
polyethylene from these $C$ values by equation 7 when the polymer film thickness in each column is known. Such a procedure is proper only if the other contributions to peak spreading discussed earlier are small when compared to that arising from polymer or stationary phase mass transfer. This assumption has been made in many previous studies of probe-polymer diffusion. However, if the other gas phase kinetic processes are operating to a significant extent then the limiting slope at high flow rate of Figure VI-l should also reflect these contributions, as indicated by equations 8 and 14 . It was important, therefore, to estimate the relative magnitude of the gas phase terms as compared to the stationary phase contribution to determine if such a simplified data analysis procedure could be applicable.

A further consideration is the nature of the carrier velocity on which the plate height depends. Equation 14 indicates that while the plate height contribution of the $B, D, E$, and $F$ terms are directly or inversely proportional to the carrier outret velocity $u_{0}$, the $C$ term contribution to $\bar{H}$ depends on the average velocity. Consequently, the often-employed plot of $\bar{H}$ vs average carrier velocity suggested by equation 2 will not be linear at high flow velocity for any variant of velocity if both stationary and gas phase mass transfer effects are nonnegligible.

The estimated gas phase contributions to peak spreading were computed for both columns and compared to the limiting slope $C$ calculated from the van Deemter equation least squares fit. It was evident that the peak spreading attributed to gas phase diffusion made a significant contribution to the overall kinetic broadening for column A. In instances of this nature, where gas phase diffusion terms make a significant contribution to peak spreading, difficulty can be encountered in deciding which are important and calculating their values. Alternatively, it is possible to resolve the gas and stationary 
phase mass transfer contributions without directly estimating the magnitude of the gas phase terms. Purnell [8] has suggested a method which involves first determining $A$ and $B$ of equation 14 and employing these values to reduce the experimental $\bar{H}$ to the peak spreading arising only from mass transfer effects, $\bar{H}^{\prime}$. In terms of an equation:

$$
\bar{H}^{\prime} / u_{0}=\left(\bar{H}-A-\frac{B}{u_{0}}\right) / u_{0}=\frac{2 p_{0}}{p_{i}+p_{0}} C+D+E+F+G
$$

When the left-hand side of this equation is plotted against the pressure correction $2 p_{0} /\left(p_{i}+p_{0}\right)$, a straight line with slope $C$ and intercept $(D+E$ $+F+G$ ) should be obtained. Evaluation of the constants $A$ and $B$ requires that experiments be conducted over a wide range of carrier gas flow velocities. Limited low velocity data often precludes experimental determination of these constants in many cases; however, they can be estimated from theoretical considerations and experimental results. The multipath $A$ term defined in equation 3 has been determined to have a $\lambda$ value between one half and unity in many cases [9] and a value of 0.5 was assumed here. The longitudirial diffusion $B$ term most often has a $\gamma$ value near one [9] and unity was utilized in the determinations. These values were used to calculate the $A$ and $B$ terms of equation 19 and a fit of $\bar{H}^{\prime} / u_{0}$ against the pressure correction term gave a linear least squares line with a slope of 16.4 ms and calcuiated standard deviation of 5.5 percent for column $A$ and $21.9 \mathrm{~ms}$ with calculated standard deviation of 6.6 percent for $B$. These results are compared to the van Deemter fit slopes in Table VI-l on the following page. The smaller standard deviations of the Purnell method slopes compared to the corresponding values for the van Deemter fit discussed earlier indicated the better applicability of the Purnell method. The $C$ value obtained by this method for column $A$ was more 
Comparison of the $C$ Coefficient Determined by Two Methods for LPE-Octadecane at $150^{\circ} \mathrm{C}$

\begin{tabular}{lccc}
$\begin{array}{l}\text { Column } \\
\text { Code }\end{array}$ & $\begin{array}{c}\text { Column Loading } \\
\text { (Mass Fraction Polymer) }\end{array}$ & $\begin{array}{c}\text {C}_{\text {van Deemter }} \\
\text { (Eq. 2) }\end{array}$ & $\begin{array}{c}\text { C Purne 11 } \\
\text { (Eq. 15) }\end{array}$ \\
\hline A & $6.10 \times 10^{-4}$ & $7.05 \mathrm{~ms}$ & $16.4 \mathrm{~ms}$ \\
B & $5.71 \times 10^{-3}$ & $18.9 \mathrm{~ms}$ & $21.9 \mathrm{~ms}$ \\
\hline
\end{tabular}

than twice that obtained from the van Deemter analysis. This was attributed to the latter's combination of the effects of polymer and gas phase mass transfer in a nonlinear manner, which can be noted by comparing the $C$ term appearing in equation 2 to its complement in equation 14, the expression in parenthesis. For the case of column $A$ where the gas phase terms contributed significantly to the peak broadening, the van Deemter equation-based analys is underestimated the magnitude of $C$. Gas phase mass transfer effects for column B contributed only slightly to the total broadening and this was reflected in the similar results of the two methods for determining $C$ which differed by only 15 percent, as indicated in Table VI-1.

While the method of determining the poiymer phase mass transfer contribution can be important under certain column conditions, the most significant parameter which affected the diffusivity determination was the distance over which diffusion occurred. This distance is commonly identified with the effective film thickness $s$ of equation 7 . It is often assumed that the stationary phase is distributed as a uniform thin film over the entire surface of the spherical glass bead column packing. The effective film thickness for this geometry can be calculated from the expression: 


$$
s=\frac{M_{s}}{4 \pi r^{2} N \rho_{s}}
$$

where $M_{s}$ is the mass of polymer phase in the column, $N$ the number of support particles in the columr, and $\rho_{s}$ the polymer density. Such a configuration requires a high amount of stationary phase surface area, however, and a . geometry in which the stationary phase is confined by capillary forces into annular regions around the contact points between support particle spheres has been advocated as a means of minimizing the exposed stationary phase surface area. This gecmetry has been observed for both low molecular weight [6] and polymeric [?0] stationary phases coated onto glass bead supports and is illustrated schematically in Figure VI-3. A stationary phase film may aiso be present on surfaces where the beads are not in contact, as indicated in the inset. Its thickness, however, should be much less than that of the stationary phase in the region of adjacent support beads.

The different diffusion path lengths expected for the two extremely different polymer distributions described above can exert a significant influence on determinations of polymer-probe diffusivity by IGC. Giddings [11] has compared the contribution to peak spreading expected from a hypothetical support coated with two regions, a thin film of uniformly adsorbed stationary phase and a second region where the liquid stationary phase collects at the support contact points. He coricludes that for glass bead supports at mass fraction loadings up to about 0.015 , the bulk of the stationary phase is present at the bead contast points and controls the plate height performance by virtue of its greater thickness. An equation has been proposed [12] to relate $C$ to measurable column parameters for this geometry and is as follows: 


$$
C=\frac{r^{2} k}{3 D_{s}(1+k)^{2}}\left(\frac{m_{s} \rho}{18.75 \rho_{s}}\right)^{1 / 2}
$$

where $\rho$ is the density of the support particles and $m_{s}$ the mass fraction of polymer phase in the column. This treatment assumes that the spherical support particles pack randomly and are surrounded by 6.25 nearest neighbors. The relation for $C$ is similar in form to equation 7 . If the differing variables are combined into an explicit expression for $s$ in terms of the particle size, the following relation is obtained:

$$
s=\left(\frac{m_{s} \rho}{18.75 \rho}\right)_{s}^{1 / 4} \frac{r}{2 \sqrt{5}}
$$

This equation provides for the calculation of a value of $s$ which, when substituted into equation 7 , yields a result for $C$ identical to the $C$ term determined by equation 17 .

A computation of $s$ by these methods was useful for comparing the effective film thicknesses of the two stationary phase arrangements considered.

The average film thicknesses calculated in the above manner for a polymer stationary phase collected at bead contact points were much greater than the thicknesses corresponding to the uniform layer geometry, as can be noted from the following table.

\section{Table VI-2}

Effective Layer Thicknesses for Columns with Differing Polymer Phase Geometry

\begin{tabular}{ccc}
\hline Column Code & $\begin{array}{c}\text { Effective Layer Thickness, } s(\mu \mathrm{m}) \\
\text { Unnular Film at } \\
\text { Surface Film } \\
\text { (Eq. 16) }\end{array}$ & $\begin{array}{c}\text { Contact Points } \\
\text { (Eq. 18) }\end{array}$ \\
\hline A & 0.043 & 5.58 \\
B & 0.404 & 9.77 \\
\hline
\end{tabular}


Both equations 7 and 17 were used to calcualte the diffusion coefficient of octadecane in HDPE. For column A assuming a thin uniform film, $D_{E}$ was calculated from the $C$ coefficient obtained with the Purnell treatment by substituting equation 16 into equation 7 ; a result of $3.8 \times 10^{-11} \mathrm{~cm}^{2} / \mathrm{s}$ was obtained. Assuming a geometry where the polymer collects at the bead contact points for column $A, D_{S}$ was calculated according to equation 17 and a value of $6.4 \times 10^{-7} \mathrm{~cm}^{2} / \mathrm{s}$ determined. These results and the corresponding values for column B are summarized in Table VI- 3 below.

Tabie VI-3

Influence of Film Geometry on the Diffusion Coefficient of Octadecane in Linear Polyethylene at $150{ }^{\circ} \mathrm{C}$

\begin{tabular}{|c|c|c|}
\hline Column Code & $\begin{array}{l}\text { Diffusion } \mathrm{Cc} \\
\text { Uniform Thin } \\
\text { Surface Film } \\
\text { (Eqs. } 7 \text { and } 16 \text { ) }\end{array}$ & $\begin{array}{l}t\left(\mathrm{~cm}^{2} \cdot \mathrm{s}^{-1}\right) \\
\text { Annular Film at } \\
\text { Contact Points } \\
\quad \text { (Eq. 17) }\end{array}$ \\
\hline $\begin{array}{l}A \\
B\end{array}$ & $\begin{array}{l}3.8 \times 10^{-11} \\
4.1 \times 10^{-10}\end{array}$ & $\begin{array}{l}6.4 \times 10^{-7} \\
2.4 \times 10^{-7}\end{array}$ \\
\hline
\end{tabular}

It was evident that the uniform thin film geometry assumption led to unreasonably low values of the diffusion coefficient. In addition, an order of magnitude discrepancy existed between results for the two columns. The assumption of a collection of the polymer stationary phase at bead contact points yielded diffusion coefficients of reasonable magnitude for the temperature of the experiment and column-to-column agreement within a factor of three. This threefold difference in the diffusivity results may be attributed to the effects of differing probe concentrations in the polymer phase of the two columns.

Diffusivity values are often quite sensitive to the concentration of the diffusing species, depending approximately either linearly or exponentially 
on concentration [13]. In these IGC determinations the probe concentration in the polymer film varied both along the column length due to increasing peak broadening with transit time and with the depth of penetration into the polymer film expected from Fickean diffusion. The diffusivity determined was therefore a concentration-averaged value over all column conditions. The actual concentration of octadecane in the polyethylene film was estimated to range from 0.01 to 1.5 percent by mass for column $A$ and from 0.001 to 0.16 percent by mass for column $B$. The diffusion coefficient calculated from the column $B$ results corresponded to probe concentrations about an order of magnitude lower than for column A. This could account for the lower vaiue of probe diffusivity determined with column B. For either a linear or exponential dependence of the diffusion coefficient on concentration, the diffusivity should be less at lower diffusant concentrations, as found here. A determination of the functional relationship of the diffusivity on diffusant concentration was not possible, however, because of the variation in probe concentration along the column length and the limited amount of data available.

Limited data are available in the literature on diffusion of the higher alkanes in LPE; however, studies of similar diffusants, linear aliphatic esters with 25 to 45 backbone units, have been conducted by Klein and Briscoe [14]. Extrapolating their experimental results to an 18 unit chain leads to an expected diffusion coefficient of $5 \times 10^{-6} \mathrm{~cm}^{2} / \mathrm{s}$ in linear polyethylene at $152{ }^{\circ} \mathrm{C}$. This result is about an order of magnitude greater than the IGC determinations for a polymer film collected at the support bead contact points.

The distribution of film thickness also plays an important role in determining the magnitude of the diffusivity. The previous calculations 
assumed that the film thickness was constant. In an actual column, howeve. the film thickness would be expected to be distributed about the mean values employed here. An alternate form of equation 7 can be used to estimate the effects of different film thicknesses [15]. The equation is:

$$
D_{s}=\frac{k}{c(1+k)^{2}} \sum_{i} q_{i} x_{i} s_{i}^{2}
$$

where $q_{j}$ is a configuration factor and $x_{j}$ the volume fraction per unit column volume of stationary phase segments with thickness $s_{i}$. Assuming a uniform configuration factor and holding $x_{i}$ constant gives:

$$
D_{s}=\frac{k q x}{c(1+k)^{2}} \sum_{i} s_{i}^{2}=z \sum_{i} s_{i}^{2}
$$

which reduces to:

$$
0_{s}=2 s^{2}
$$

for a uniform film of thickness $\mathrm{s}$. For $\exists$ distribution with an equal volume of film $0.1 \mathrm{~s}$ and $10 \mathrm{~s}$ in thickness:

$$
D_{s}^{\prime}=z\left[5(0.1 s)^{2}+0.05(10 s)^{2}\right]=5.05 \mathrm{Zs}^{2}
$$

This distribution, resulting from the same volume of stationary phase present in the uniform thickness case, leads to a diffusivity value five times larger than that corresponding to a uniformly distributed film. Table VI-4 summarizes the effects of different thickness distributions on the diffusion coefficient $D_{S}{ }^{\prime}$ compared r.s that expected from a uniform film of thickness $5, D_{S}$. All the geometries considered require the same volume of stationary phase per unit column volume and have an average thickness of $s$. It is evident from these examples that thick sections of the polymer film 
Table VI-4

Effect of Thickness Distribution on Diffusivity in the Polymer Phase

\begin{tabular}{|c|c|c|}
\hline $\begin{array}{l}\text { Thin Section } \\
\text { Depth }\end{array}$ & $\begin{array}{c}\text { Thick Section } \\
\text { Depth }\end{array}$ & $D_{s}^{1} / D_{s}$ \\
\hline $0.50 \mathrm{~s}$ & $2 s$ & 1.25 \\
\hline $0.25 \mathrm{~s}$ & $4 s$ & 2.13 \\
\hline $0.10 \mathrm{~s}$ & $10 \mathrm{~s}$ & 5.05 \\
\hline $0.02 \mathrm{~s}$ & $50 \mathrm{~s}$ & 25.01 \\
\hline $0.01 \mathrm{~s}$ & $100 \mathrm{~s}$ & 50.01 \\
\hline
\end{tabular}

can exert a disproportionate influence and lead to a considerable increase in the polymer-probe diffusion coefficient when compared to the value calculated for an IGC experiment with an assumed ideal polymer layer of uniform thickness. An experimentally difficult determination of the variability of the polymer film in the IGC column would be necessary in order to correct the calculated diffusivity values for the actual polymer layer nonuniformity.

\section{Experimental Details}

The column used throughout the relative diffusivity study was prepared from glass support beads coated with a layer of the linear polyethylene (LPE) NBS SRM 1475 and packed into a $6.35 \mathrm{~mm}$ outer diameter stainless stee 1 tube, as described in the previous annual report [1]. After installation in the gas chromatograph, a final drying and column conditioning step was carried out by purging with dry, deoxygenated helium followed by heating to $150^{\circ} \mathrm{C}$ for a 24-hour period. The column used is identical to column B in the previous annual report and contains $5.71 \times 10^{-3}$ mass fraction of LPE. It was chosen because of the relatively small contribution which gas phase mass transfer made to the overall peak spreading when compared to column $A$ of that report. 
Several alkane probes and an antioxidant were employed as diffusants and are listed in the table below [16]. All were dissolved in hexane (Burdick and Jackson Labs.) to give solutions of about $0.02,0.2$, and 2.0 percent by mass.

\section{Table VI-5}

Probes Employed in an IGC Study of Relative Diffusivity in LPE

\begin{tabular}{lll}
\hline Probe & Code & Source \\
\hline n-octadecane & $\mathrm{C}_{18}$ & Supelco \\
n-hexadecane & $\mathrm{C}_{16}$ & Aldrich Chemical \\
2-methylpentadecane & $2 \mathrm{MeC}_{15}$ & Alfa Products \\
$\begin{array}{l}\text { 2,2,4,4,6,8,8- } \\
\text { heptamethylnonane }\end{array}$ & $7 M e C_{9}$ & Aldrich Chemical \\
$\begin{array}{l}\text { n-tridecane } \\
\begin{array}{l}\text { 1,3-di-tert-buty1-2:- } \\
\text { hydroxy-5-methylbenzene }\end{array}\end{array}$ & $\mathrm{C}_{13}$ & American Petroleum Institute \\
\hline
\end{tabular}

Approximately $1 \mathrm{Al}$ of each solution was injected into the flash vaporization injector of a gas chromatograph, maintained at $180^{\circ} \mathrm{C}$. The column oven was held at $150{ }^{\circ} \mathrm{C} \pm 0.3{ }^{\circ} \mathrm{C}$ throughout. The eluted peaks were monitored with a flame ionization detector (FID) held at $190^{\circ} \mathrm{C}$, whose signal at $10^{-10} \mathrm{~A} / \mathrm{mV}$ sensitivity was fed to an electronic integrator connected to a strip chart recorder and to a high acquisition speed recorder-plotter. The former setup was employed to time the peak maxima to within $0.01 \mathrm{~min}$ and for manual measurements of the chart width at half maximum at chart speeds up to $1 \mathrm{~cm} / \mathrm{s}$. Typical results are shown in Figure VI-4.

The rapid collection capabilities of the high speed recorder-plotter allowed the FID output to be sampled at $10 \mathrm{~ms}$ intervals, facilitating analysis 
of the marker and solvent peaks. A typical trace is shown in Figure VI-5 for five repeated determinations. Data collection was triggered upon injection of the methane and hexane vapor sample and the time to the methane peak maximum, assumed to have $k=0$, was employed to determine $t_{M}$ of equation 6 and to calculate the column average linear flow velocity, $\bar{u}$, from the expression:

$$
\bar{u}=L / t_{M}
$$

The quantity of interest, the column outlet velocity, was then calculated from $\bar{u}$ as follows:

$$
u_{0}=\frac{2 \bar{u}}{3}\left[\frac{\left(p_{i} / p_{0}\right)^{3}-1}{\left(p_{i} / p_{0}\right)^{2}-1}\right] .
$$

Column inlet and outlet pressures were monitored with calibrated pressure gauges of appropriate range. The outlet pressure was very near to atmospheric in all cases. The helium carrier gas volumetric flow rate was measured with a soap film flow meter, when necessary. The data were collected over a corrected flow rate range of about 1 to $5 \mathrm{ml} / \mathrm{s}$.

The first derivative of a chromatographic peak with respect to time can be used to aid in determinations of the maximum height and peak widths indicated in Figure VI-I. At a point where the chromatogram is at a maximum, the first derivative will be zero while the second derivative is negative. Determination of the first derivative zero point is illustrated with curve $B$ of Figure VI- 6 , the first derivative of curve $A$ obtained by experiment. The inflection points of the chromatogram are also readily indicated by maxima or minima in the derivative curve. The magnitude of the derivative at this point gives the slope of a tangent to the peak. The ratio of the absolute values of the derivative maximum and minimum gives a measure of peak symmetry. 
For the purposes of this study, a symmetric peak is defined as that having a front to rear tangent slope ratio of $1 \pm 0.2$. The highly asymmetric peaks that result from excessive probe concentrations or adsorption, two of the many factors which can cause deviations from Henry's law in the chromatographic column, are not amenable to analysis by the methods outiined here for Gaussian-shaped peaks. The time period between maximum and minimum of the derivative curve can be measured easily and is equal to twice the standard deviation of the peak, as illustrated in Figures VI-1 and VI- 6 . The above procedure was used to collect information on the center position, standard deviation, and symmetry of the eluted diffusant peaks.

\section{Relative Diffusivity Determinations}

As noted earlier, the probes 1 isted in Table VI-5 were studied in the same LPE-containing column. This allows the diffusivity of one probe in LPE to be compared to that of another, irrespective of the method of any calculation of the absolute diffusivity of the probes in the polymer. The experimental data acquired for tridecane in LPE is given in Table VI- 6 and can be used to give an indication of typical values of the various parameters utiiized in these determinations. The variables $p_{j}, p_{0}$, and $t_{M}$ are used in the calculation of $u_{c}$ by equations 23 and 24 . The plate height is determined from $w_{\frac{1}{2}}$ and $t_{R}$ with equation 1 or from $w_{i}$ and $t_{R}$ by the following expression:

$$
H=\frac{L}{4}\left(\frac{w_{i}}{t_{R}}\right)^{2}
$$

When values of $A$ and $B$ are available, the plot suggested by equation 15 can be constructed from the data of Table VI-6. The previous annual report [1] contains a more detailed example of the calculation of these two constants from equations 3 and 4 than the abbreviated discussion given here. The values used in calculating $\bar{H}^{\prime} / u_{0}$ are given in a footnote to Table VI-6. 


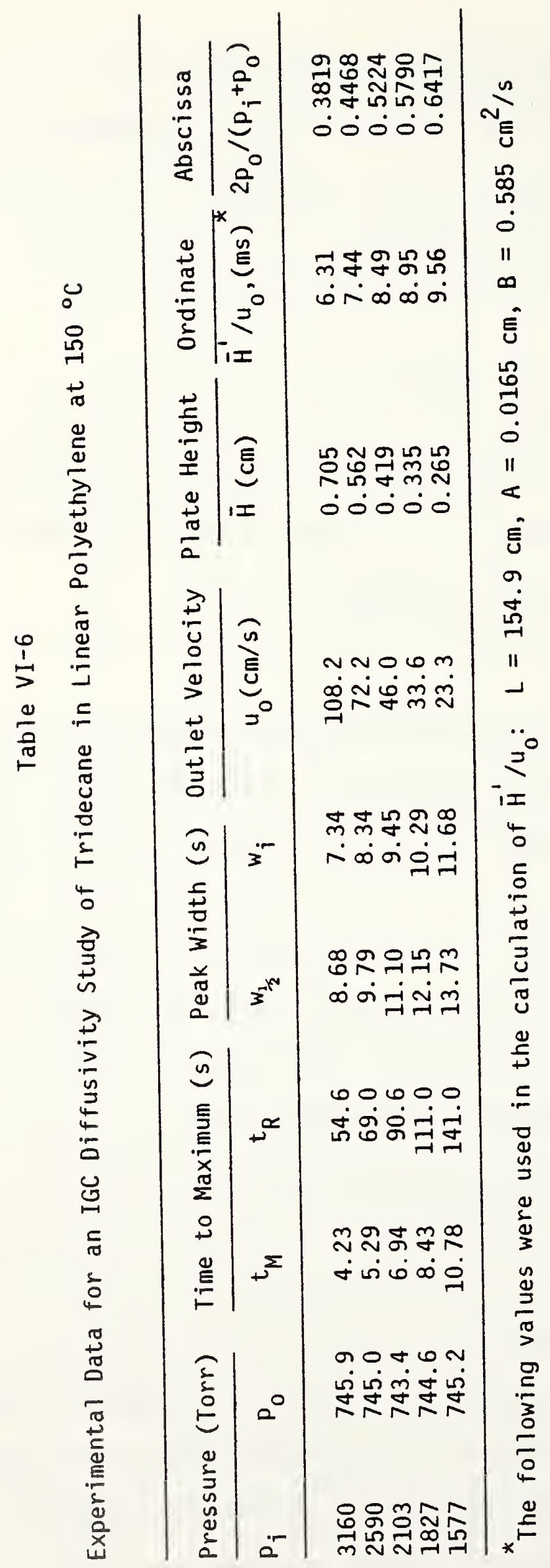


Plots of $\bar{H}^{\prime} / u_{0}$ against the $2 p_{0} /\left(p_{j}+p_{0}\right)$ term are shown for five diffusants in Figure VI-7. The slopes of these plots provide a value for the $C$ constant needed in a dirfusivity determination. These $C$ values are listed in Table VI-7, accompanied by the corresponding value of $k$ for each

Table VI-7

Relative Diffusivity Results for Several Alkanes and BHT in LPE at $150{ }^{\circ} \mathrm{C}$

\begin{tabular}{lrrrcc}
\hline Probe & $C(\mathrm{~ms}) \pm \mathrm{C}(\%)$ & $k \pm \sigma(\%)$ & $D_{\text {Probe }}$ & $D_{\text {Probe }}{ }^{1 D} C_{18}$ & $D\left(10^{7} \cdot \mathrm{cm}^{2} / \mathrm{s}\right)$ \\
\hline$C_{18}$ & $21.9 \pm 7$ & $119 \pm 3$ & $0.38 Y$ & 1 & 2.4 \\
$C_{16}$ & $7.8 \pm 14$ & $60.9 \pm 1$ & $2.05 Y$ & 5.4 & 13 \\
$2 \mathrm{MeC}_{15}$ & $8.7 \pm 12$ & $49.0 \pm 1$ & $2.25 Y$ & 6.0 & 14 \\
$7 \mathrm{MeC}_{9}$ & $18 \pm 11$ & $12.8 \pm 1$ & $3.74 Y$ & 9.9 & 24 \\
$\mathrm{C}_{13}$ & $12 \pm 9$ & $12.1 \pm 1$ & $5.74 Y$ & 15.2 & 36 \\
$\mathrm{BHT}$ & $9.9 \pm 11$ & $32.4 \pm 2$ & $2.94 Y$ & 7.8 & 19 \\
\hline
\end{tabular}

probe as calculated from the data of Table VI- 6 by equation 6 . This information can then be used to determine a value for the relative diffusivity by the following procedure. Equation 17 can be rearranged to collect all the variables used only to characterize the polymer packing into one term and solved for the diffusivity. The following expression results when this is done:

$$
D_{s}=\frac{k}{C(1+k)^{2}}\left(\frac{r^{4} m_{s} \rho}{168.75 \rho_{s}}\right)^{\frac{1}{2}}
$$

The first term in the above equation contains the variables which characterize the interactions between the probe and polymer while the square room term consists of variables which describe polymer layer and support 
particle characteristics only. These latter variables are constant throughout all determinations with this particular column and this collection of parameters can be abbreviated as the constant $Y$. The following version of equation 26 is consequently obtained:

$$
D_{s}=\frac{k}{c(1+k)^{2}} \cdot Y
$$

This equation was used to determine the probe diffusivity values given in the fourth column of Table VI-7. If one of the probes is selected as a reference, in this instance octadecane, the diffusivity of a probe in the polymer relative to the diffusivity of the reference probe in that polymer can be obtained from a ratio of the respective results from equation 27 . These values are given in the fifth column of Table VI-7.

An alternate determination of the relative diffusivity might be based on equation 7. In this instance the following expression would be obtained for $D_{\varsigma}$ :

$$
D_{s}=\frac{k}{c(1+k)^{2}} \cdot \frac{2 s^{2}}{3}=\frac{k}{c(1+k)^{2}} \cdot Y^{\prime}
$$

Equations 27 and 28 are identical except for the constants $Y$ and $Y^{\prime}$, which are derived from different collections of parameters used to describe the distribution of the polymer phase on the support. The numerical front-factor for $Y^{\prime}$ is identical to that given for $Y$ in column four of Table VI-7 since it depends only on the values of $C$ and $k$, which are the same for either method. The relative diffusivity results would also be identical as the $Y$ or $Y^{\prime}$ constant is eliminated by the ratioing method used to obtain this quantity.

An absolute diffusion constant for each probe in the polymer can readily be obtained from the relative diffusivity values if an absolute diffusion coeffi- 
cient for any of the probes in that polymer exists. Taking the previously cited value in Table VI-3 for column $B$ of $2.4 \times 10^{-7} \mathrm{~cm}^{2} / \mathrm{s}$ for octadecane in LPE at $150{ }^{\circ} \mathrm{C}$ as accurate, the absolute diffusivities in column six of Tab?e VI-7 were otained by multiplying the relative diffusion coefficients of column five by this factor.

\section{Future Results}

Results on lower molecular weight alkanes, principally isomers of octane and normal decane and tridecane, at temperatures below the LPE melting point will be given in a later report. They will allow a more rigorous evaluation of the IGC relative diffusivity technique as many more absolute difflisivity determinations have been conducted with these compounds. Independent values for the absolute diffusion coefficient of tridecane in LPE will then be available at two temperatures, one below, and one above the polymer melting point. By using an appropriate activation energy to extrapolate one of the results and making allowances for the expected effects of polymer crystallinity on the diffusion coefficient, a direct comparison of the two results will be possible. Unfortunately, it is not possible to study the alkanes from octane to octadecane at a single temperature due to the great differences in volatility among these compounds. This necessitates the temperature extrapolation for a common probe described above as a means of connecting the two bodies of data collected at different temperatures and allowing for a critical evaluation of the method. Additional results for BHT at lower temperatures will also be provided. 
.

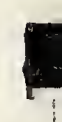

.

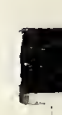

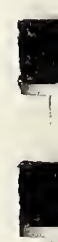

.

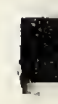

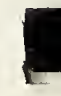

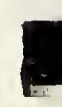

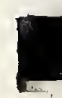

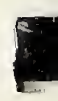

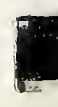

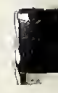

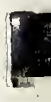

,

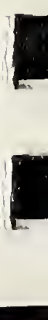




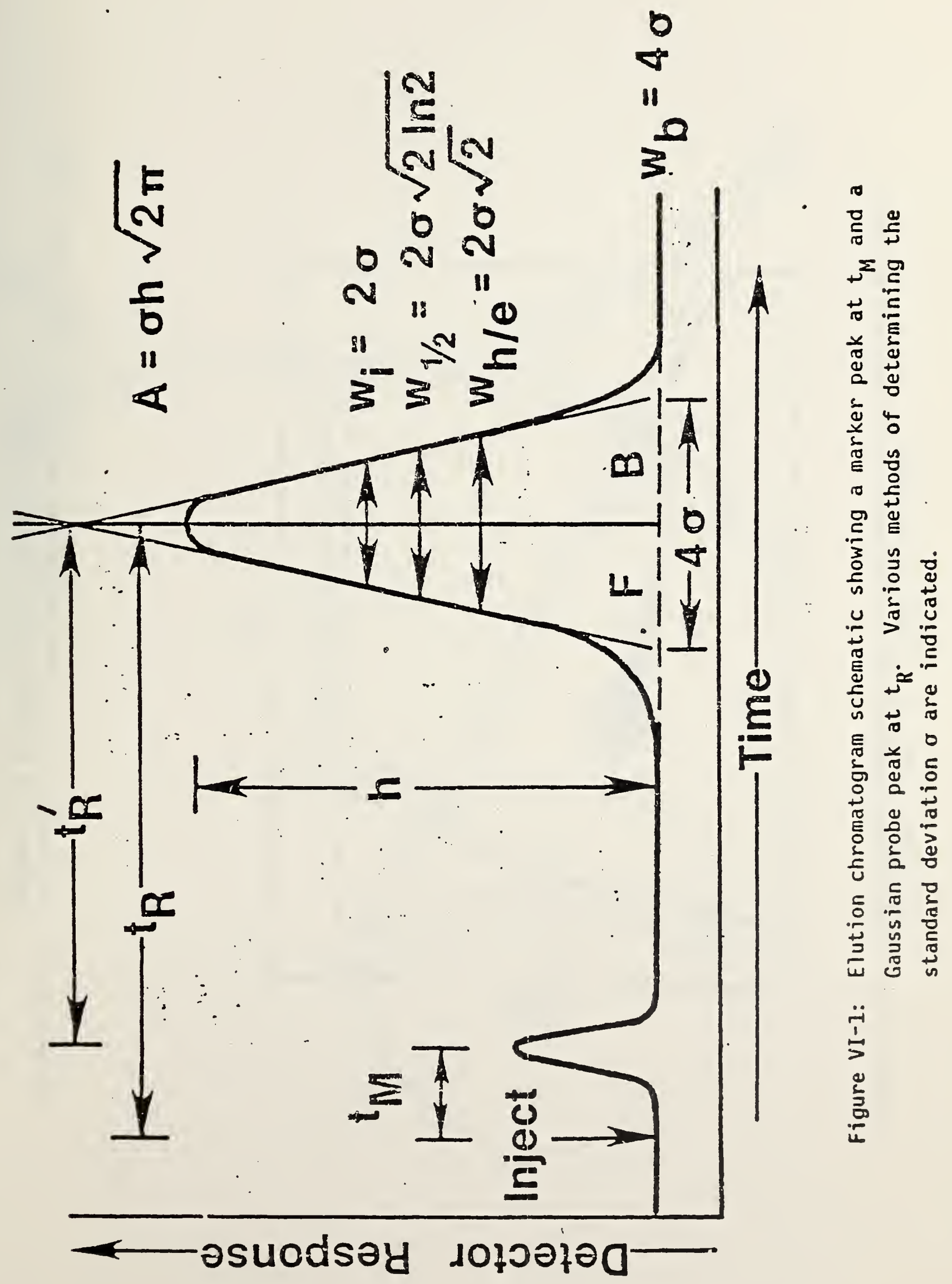



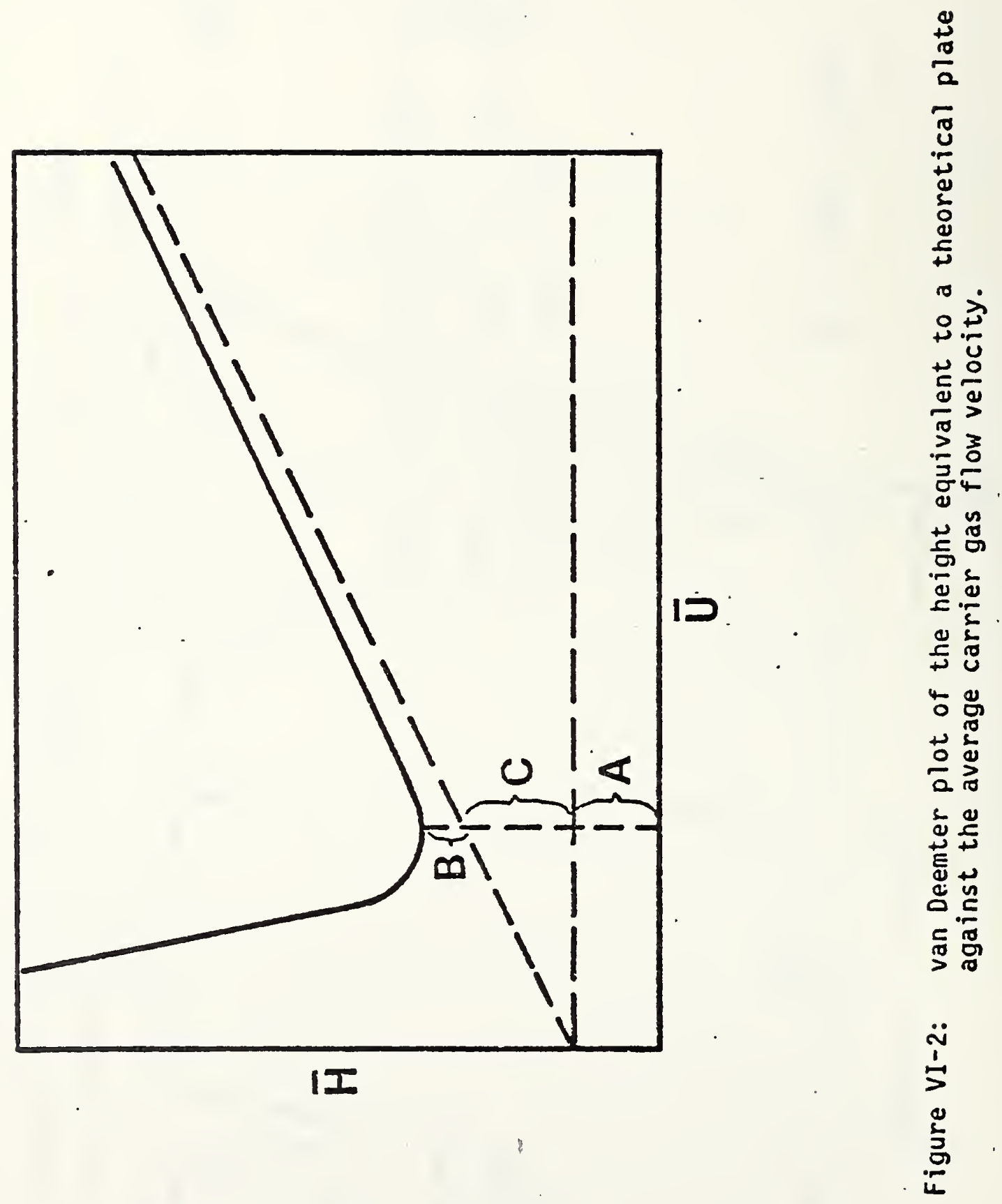


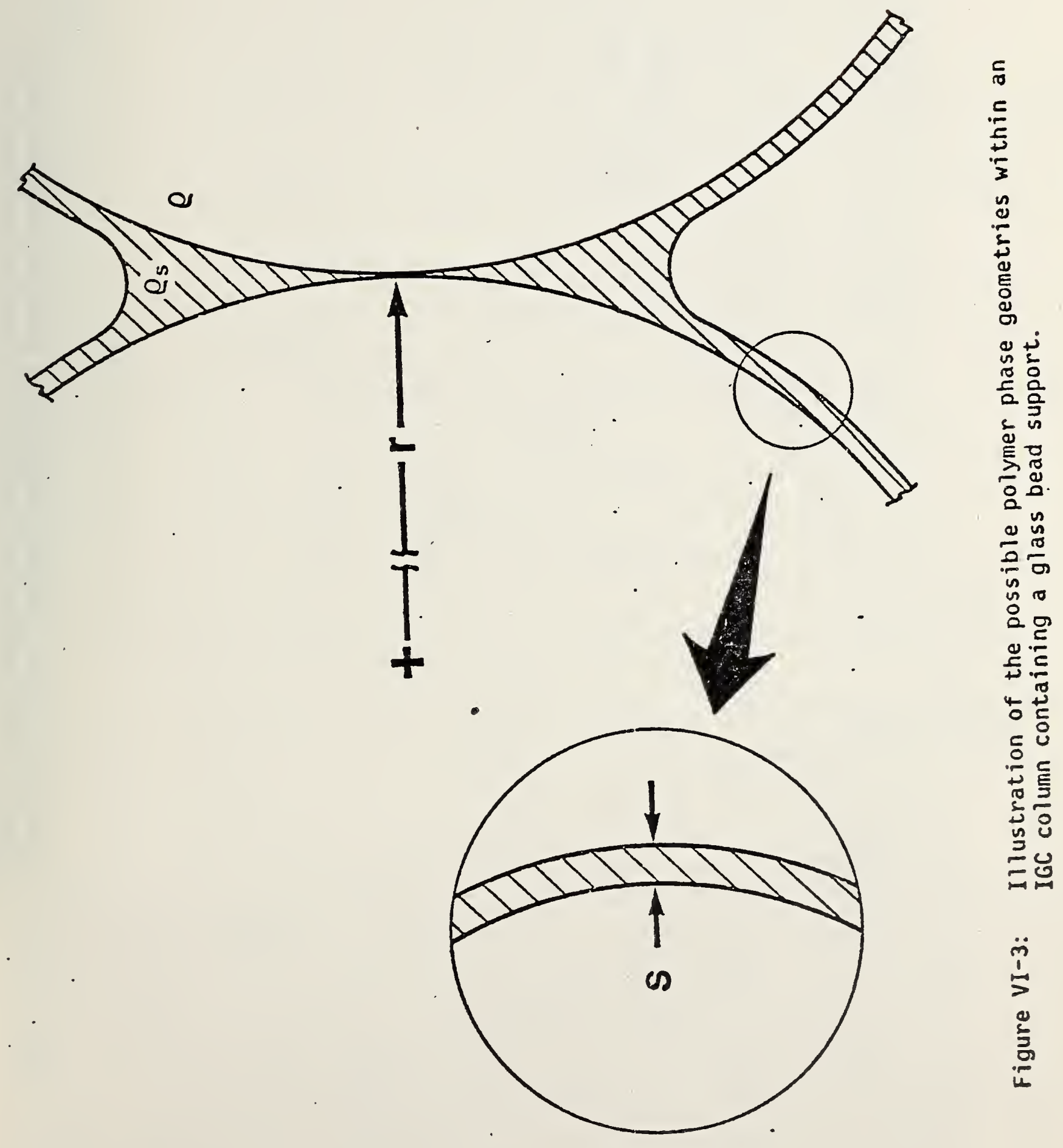




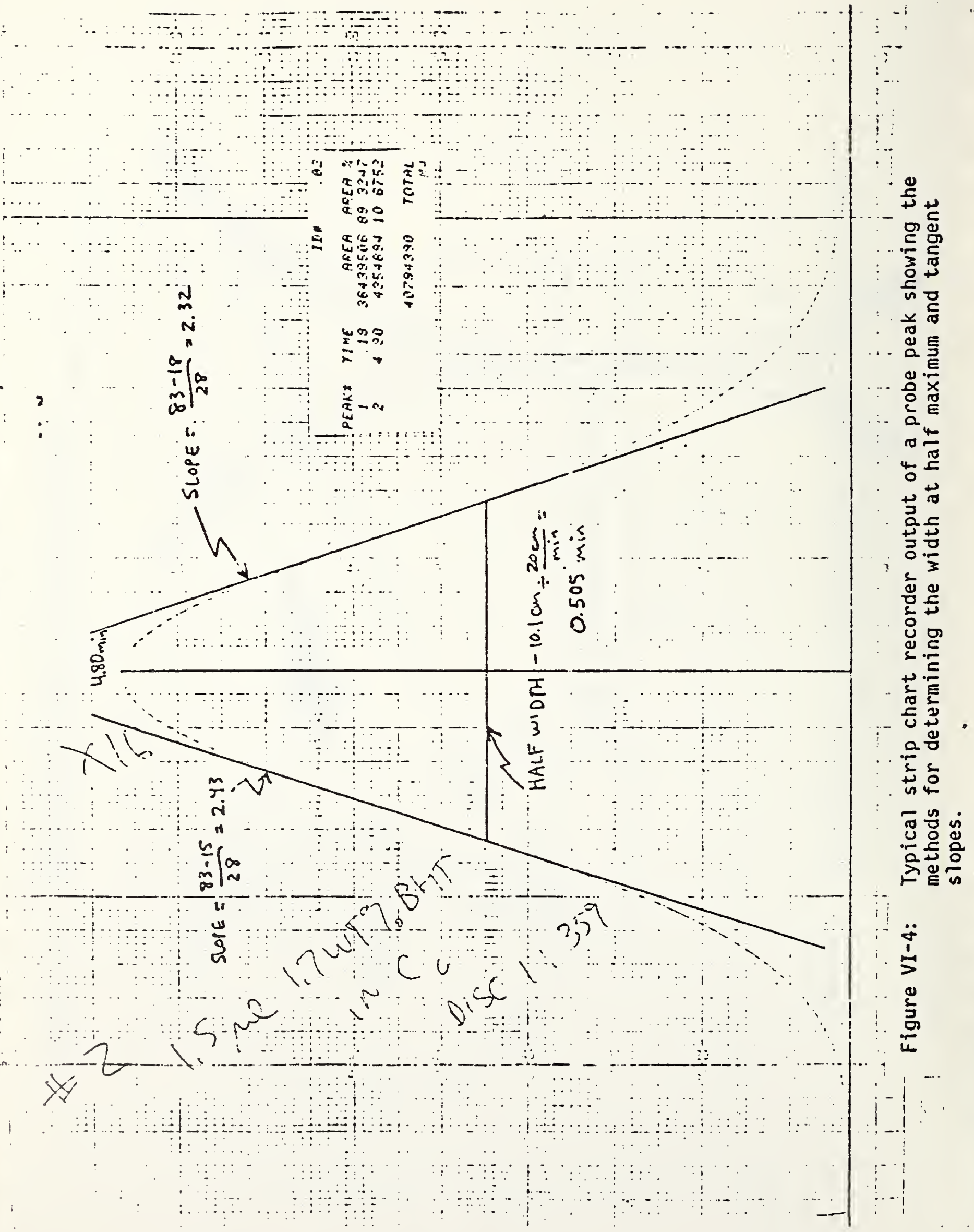




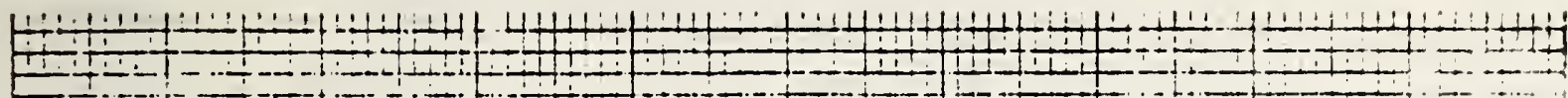

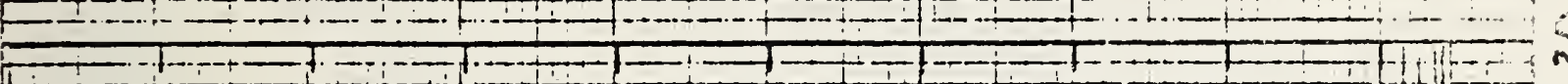

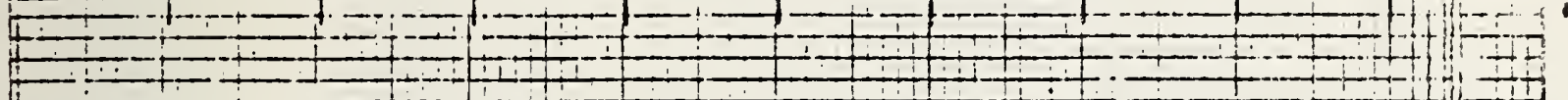

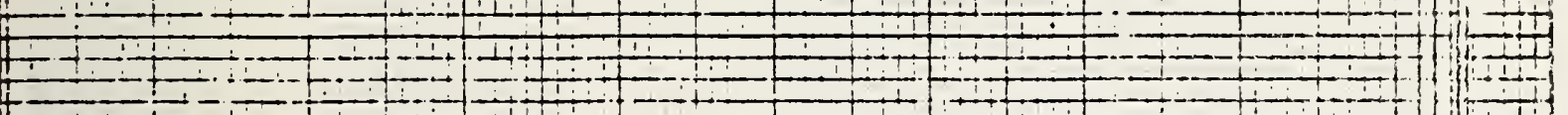

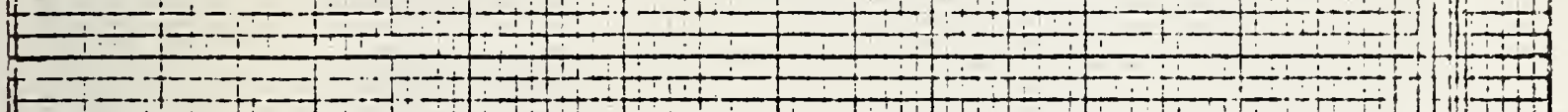

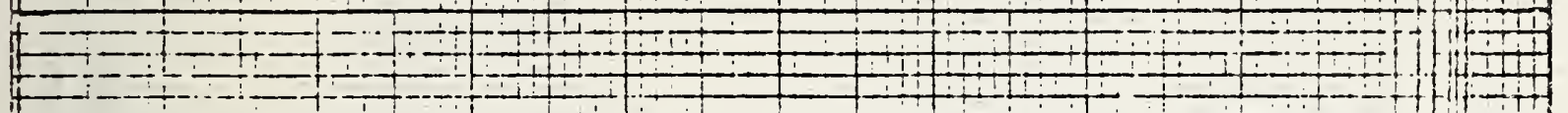

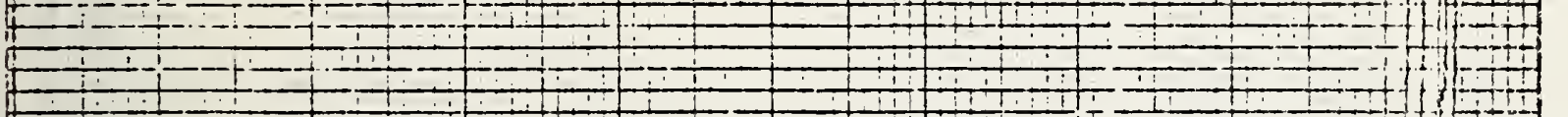

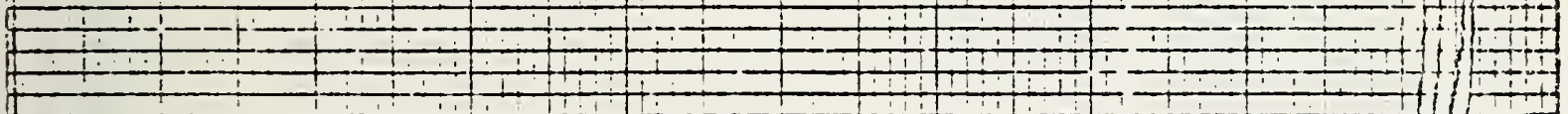
\begin{tabular}{l}
$0+1$ \\
\hline+ \\
\hline+1
\end{tabular}

+1
$+\infty$
0

LC+1

+1
$+\infty$
0

+1
$+\infty$
0

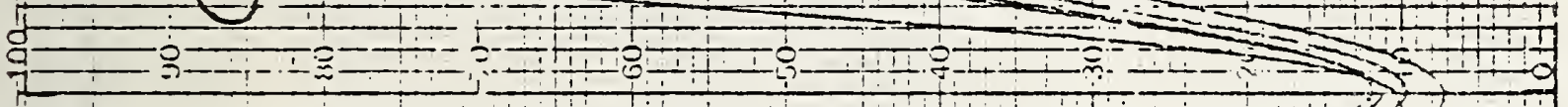

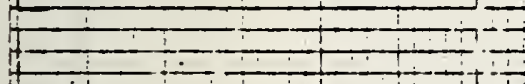
$\frac{1}{v+1}$

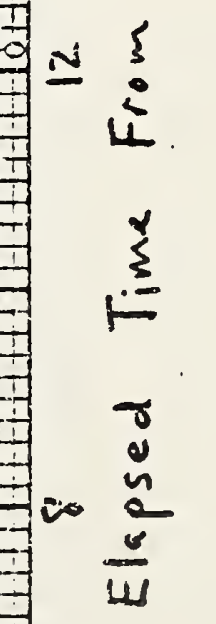

은

$+$

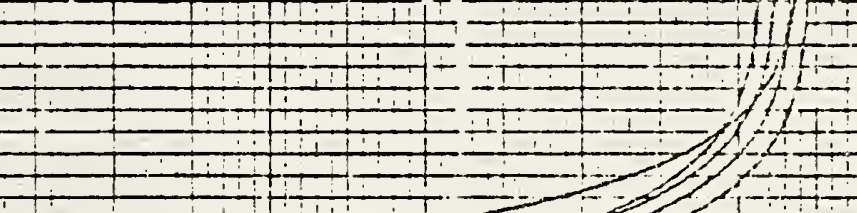




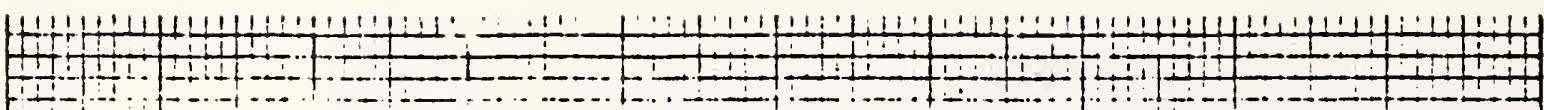

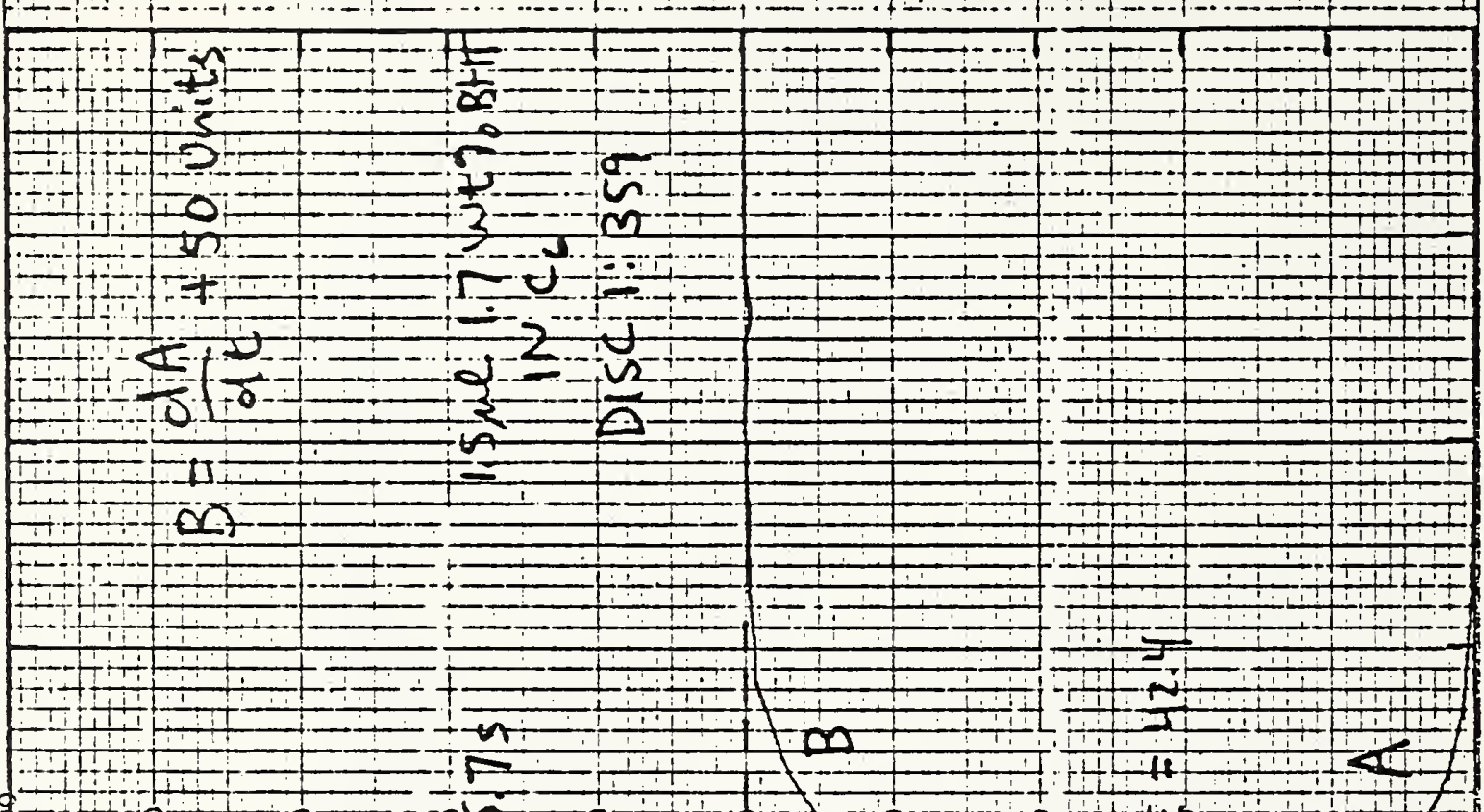
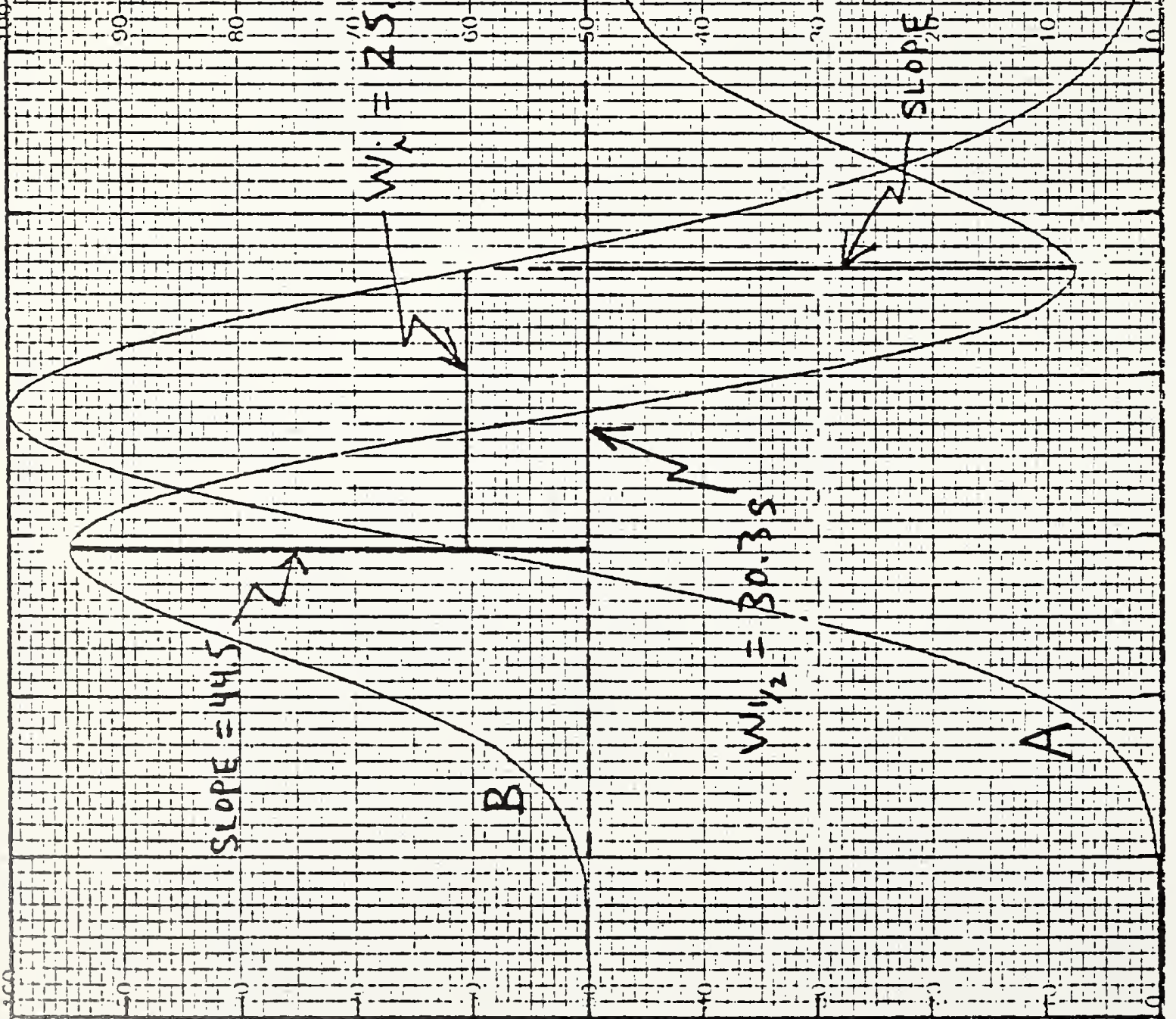


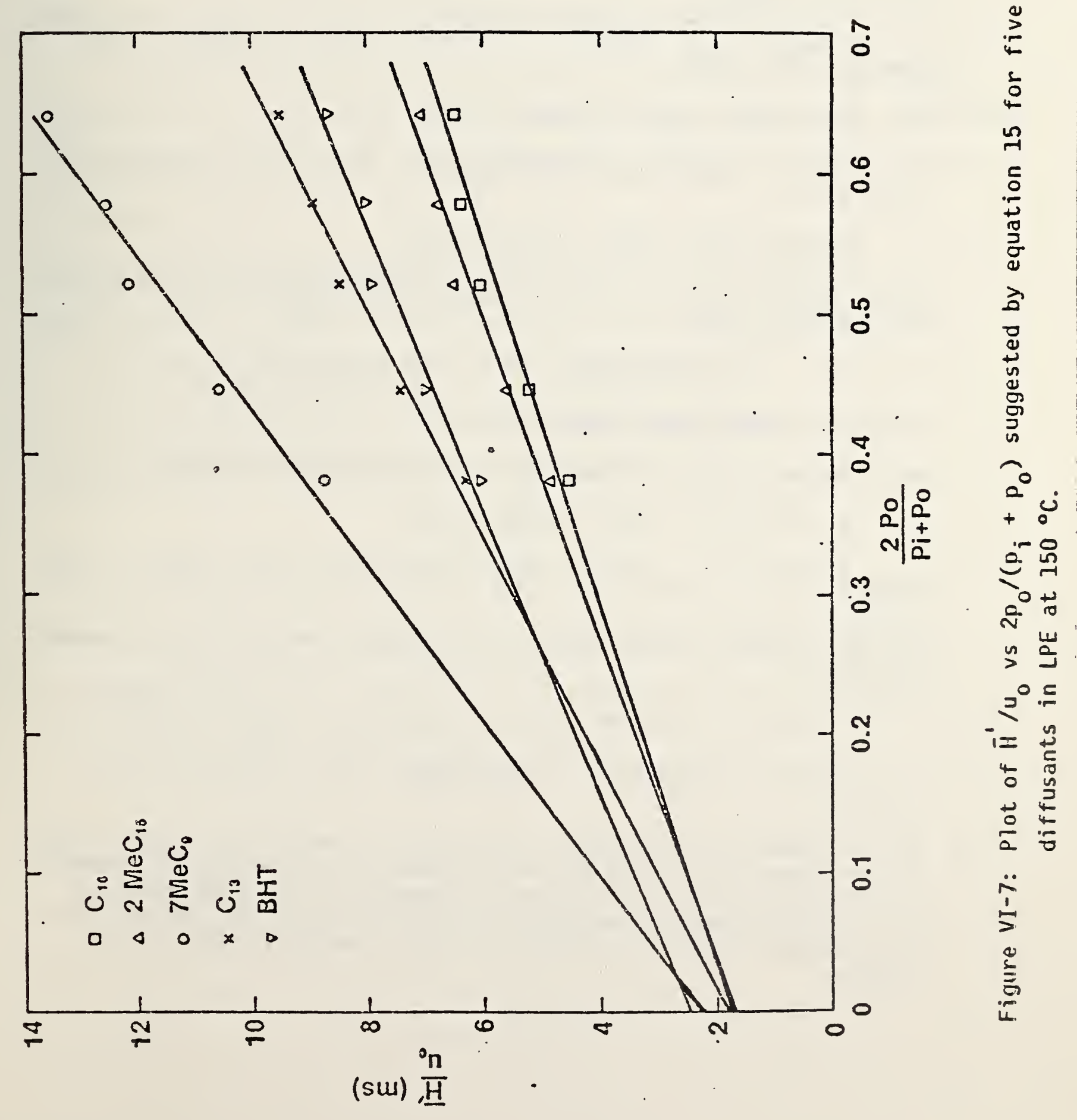


1. L. E. Smith, S. S. Chang, F. L. McCrackin, G. A. Senich, and F. W. Wang, "Models for the Migration of Low Molecular Weight Additives in Polyolefins," NBSIR 81-2264, April 1981.

2. J. J. van Deemter, F. J. Zuiderweg, and A. Klinkenberg, Chem. Eng. Sci. $\underline{5}, 271$ (1956).

3. J. C. Giddings, S. L. Seager, L. R. Stucki, and G. H. Stewart, Anal. Chem. 32, 867 (1960).

4. W. L. Jones, Anal. Chem. 33, 829 (1961).

5. J. C. Giddings, Oynamics of Chromatography, Marcel Dekker, New York (1965) Ch. 2 .

6. J. C. Gidaings, Anal. Chem. 35, 439 (1963).

7. S. Dal Nogare and R. S. Juvet, Gas-Liquid Chromatography, Interscience, New York (1962) Ch. 5.

8. H. Purne11, Gas Chromatography, Wiley, New York (1962) Ch. 8.

9. H. Purne11, Gas Chromatography, Wiley, New York (1962) Ch. 9.

10. G. J. Courval and D. G. Gray, Can. J. Chem. 54, 3496 (1976).

11. J. C. Giddings, Ana1. Chem. 34, 458 (1962).

12. J. C. Giddings, K. L. Mallik, and M. Eikelberger, Anal. Chem. 34, 1026 (1962).

13. J. Crank and G. S. Park, Diffusion in Polymers, Academic Press, London (1968) p. 17.

14. J. Klein and 3. J. Briscoe, Proc. R. Soc. Lond. A. 365, 53 (1979).

15. J. C. Giddings, Dynamics of Chromatography, Marcel Dekker, New York (1965) p. 141 .

16. Certain commercial equipment, instruments, or materials are identified in this paper in order to adequately specify the experimental procedure. Such identification does not imply recommendation or endorsement by the National Bureau of Standards, nor does it imply that the materials or equipment identifies are necessarily the best available for the purpose. 


\section{A Simplified Migration Decision Tree}

One of the most important parameters in indirect food additive regulation is the maximum al lowable concentration or a critical concentration of the species of concern in food, $C^{*}$. This value is based upon toxilogical studies and from the fractional amount of total food consumed, that would be in contact with the packaging material of concern.

Acceptable migration would be the case in which an upper bound level of migration for the maximum time and temperature for storage and usage, based upon either actual tests of sound estimations, is less than the critical or threshold level.

To this end, we have exemplified and simplified our decision tree described earlier (NBSIR 79-1779) as a flow chart shwon in Figure VII-1. 1. Load Limited Migration

In the ultimate case, all the migrant of concern moves from the packaging material into food. The concentration of the migrant in food is:

$$
c=\frac{V_{p} C_{0}}{V_{s}}
$$

where $C_{0}$ is the original concentration of the migrant in the packaging material, $V_{p}$ and $V_{s}$ are the volumes of the packaging material and food or solvent respectively. Migration is acceptable if this concentration is less than the critical concentration, $c^{*}$. 
In general, $10 \mathrm{~g}$ food is considered in contact with $1 \mathrm{in}^{2}\left(6.45 \mathrm{~cm}^{2}\right)$ of the packaging material (CRF Title 21, Part 21, Part 177). In other words, by approximating the density of foods as $1 \mathrm{gcm}^{-3}$, the ratio of the volumes of the packaging material to food $V_{p} / V_{s}$ is 0.645 \& where $\&$ is the thickness of the packaging material in $\mathrm{cm}$. Commonly this ratio is less than 1:40 or the thickness of the packaging material is less than $0.04 \mathrm{~cm}$.

Therefore, the migration level is acceptable if the following conditions are met:

$$
C_{0}<\frac{V_{s}}{V_{p}} C^{*} \quad \text { for all cases, }
$$

or

$$
c_{0}<\frac{1.53}{\ell} C^{*}
$$

if no less than $10 \mathrm{~g}$ of food

is in contact with $6.45 \mathrm{~cm}^{2}$ of packaging materiat,

or

$$
c_{0}<40 C^{*} \text { If } V_{p} \text { is less than } 1 / 40 \text { of } V_{s} \text {. }
$$

2. Solubility Limited Migration

For the case in which the loading of the migrant of concern is greater than the above limit, the saturation solubility $C_{\text {sat }}$ of the migrant in the particular type of food or food simulant is examined in order to find out whether the saturation solubility is less than the critical concentration. Special attention should be given to cases where surfactant or emulsification action may tend to increase the actual migrant concentration in food beyond the saturation concentration. The migration level is acceptabe if

$$
c_{\text {sat }}<c^{*} \text {. }
$$




\section{Partition Limited Migration}

If both the loading and the solubility exceed the limiting trireshold, then the partitioning of the migrant between the packaging material and the food or the simulant should be examined. The partition coefficient $k$ is defined as the quotient of the activities, a, of the migrant in the food and in the polymer at equilibrium. It may also be approximated by the ratio of the solubilities in the solvent and in the polymer,

$$
k=\frac{1}{k}=\frac{a_{s}}{a_{p}} \cong \frac{C_{\text {sat }}}{C_{p, s a t}}
$$

By defining $a$ ats the ratio of the amount of migrant in the solvent, $M_{\infty}$, versus that in the polymer, $M_{p_{\infty}}$, at equilibrium

$$
\alpha=\frac{M_{\infty}}{M_{p \infty}}=\frac{V_{s}}{V_{p}} k=\frac{V_{s} C_{\infty}}{V_{p} C_{p \infty}},
$$

then the final fraction of original amount of additive migrated,

$$
\frac{M_{\infty}}{M_{G}}=\frac{\alpha}{1+\alpha}
$$

where $M_{0}$ is the original loading of migrant in the polymer. For the final concentration in food or solvent to be less than the threshold,

or

$$
\begin{gathered}
c_{\infty}=\frac{M_{\infty}}{V_{s}}<C^{*}, \\
\frac{\alpha}{1+c} \frac{V_{p}}{V_{s}} C_{0}<C^{*},
\end{gathered}
$$

it requires that

$$
c_{0}<\left(\frac{V_{s}}{V_{p}}+k\right) c^{*} .
$$

Thus step 1 is just a special case of step 3. 
In the case that the partition coefficient or the saturation concentration of the migrant in the polymer is unknown or difficult to determine, the partition coefficient may be assigned a value as in our previous reports (NBSIR 79-1598 and 79-1779)

$$
\text { lnk }=\text { lnC } \text { sat }_{\text {sat }}+x_{p}^{\infty}+1=\text { lnC } \text { sat }_{\text {san }}+2
$$

In general, $x_{p}^{\infty}$ is in the range of 0 to 2 . Thus
$k<20$ sat.

This assigns a solubility of $C_{p}$, sat of $>0.05$. Therefore the requirement is simplified as,

or

$$
\begin{gathered}
C_{0}<(K+40) C^{*}, \\
C_{0}<\frac{1}{20 C_{\text {sat }}}+40 \quad C^{*}
\end{gathered}
$$

However, this simplification is applicable only when the saturated solubility in the food or solvent is relatively low and the solubility in the polymer relatively high.

4. Diffusion Limited Migration

When the equilibrium properties such as the additive loading in the polymer, the solubility of the additive in the solvent, and the partitioning of the additive between the polymer and solvent all yield too high a finai concentration of the additive in the food or the solvent beyond the threshold concentration $C^{*}$, then it is necessary to find out kineticaliy whether, under the particular storage condition and in the limited storage time, the amount of the additive migrated would produce an additive concentration in the solvent less than that of the threshold limit. The kinetics of migration depends upon additive, polymer, and solvent combinations, as well as effects of temperature and stirring. The combination of additive, polymer, and solvent or food is the most important factor influencing the diffusion coefficient. This diffusion coefficient is also dependent upon temperature, with an activation 
energy in the order of $10-20 \mathrm{kcal} / \mathrm{mol}$ for polymers in the rubbery state. A further relatively minor influence in comparison to the above factors may be observed due to the changes in the degrees of crystallinity and molecular weights of the polymer. Stirring speeds up the approach of an equilibrium condition.

If the migration is kinetically limited, i.e. much less than half of additive is migrated, then we are only interested in the short time behavior; otherwise, the estimation of total migration from step 1 would be sufficient.

For a single-sided extraction, at $t$ when $M_{t} / M_{\infty}<0.5$, we may apply the simple equation

$$
\frac{M_{t}}{M_{\infty}}=2\left(\frac{D t}{\pi}\right)^{1 / 2} \frac{1}{l}
$$

In other words

or

$$
\begin{aligned}
& \frac{M_{t}}{A}=2\left(\frac{D t}{\pi}\right)^{l / 2} C_{0}, \\
& C_{t}=2\left(\frac{D t}{\pi}\right)^{1 / 2} \frac{A}{V_{S}} C_{0},
\end{aligned}
$$

where $\ell$ is the thickness of the film and $A$ is the area of "contact. For an upper limit, $D$ is assigned the value of the diffusion coefficient measured on the polymer swollen by the solvent concerned. In the upper bound case, all migrants are assumed to be able to migrate from the polymer into solvent, i.e., $M_{\infty}=M_{0}=V_{p} C_{0}$. If partitioning exists, $M_{\infty}$ should be reduced from $M_{0}$ according to that described in step 3 .

By assuming a storage time, $t$, of 1 year $\left(3.15 \times 10^{7} \mathrm{~s}\right)$ and a container thickness of $0.04 \mathrm{~cm}$ (calculated from a food to container contact area of $10 \mathrm{~g} / \mathrm{in}^{2}$ and a container to food weight ratio of $1: 40$ ), one may obtain

$$
\begin{aligned}
& M_{t} / M_{\infty} \cong 1.6 \times 10^{5} D^{1 / 2}, \\
& M_{t} / A \cong 6300 D^{1 / 2} C_{0},
\end{aligned}
$$

or 
or

$$
C_{t} \cong 4100 D^{1 / 2} C_{0} \text {. }
$$

For $M_{t} / M_{\infty}=0.5$, a diffusion coefficient of $D=10^{-11} \mathrm{~cm}^{2} \mathrm{~s}^{-1}$ is required to meet the threshold limit. Therfore, with the above mentioned storage conditions, only in the cases where $D$ is less $10^{-11} \mathrm{~cm}^{2} \mathrm{~s}^{-1}$ will diffusion limited migration be important enough to be considered. Otherwise equilibrium conditions should be used for estimation. For thinner packaging materials, $D$ should be even less or at less than $6.2 \times$ $10^{-9} \ell^{2} \mathrm{~cm}^{2} \mathrm{~s}^{-1}$ in order for the kinetic criteria for migration to apply.

For polyolefins, the glass transition temperature is lower than $-20{ }^{\circ} \mathrm{C}$. Hence, the semicrystalline solid polymers are, in general, utilized in the condition of mixed rubbery (amorhpous portion) and crystalline states. It is usually assumed that the additive migration occurs in the amorphous region. The diffusion coefficients of migrant for the rubbery state are generally many orders of magnitude higher than those for the glassy state. For ol igomers up to $n-\mathrm{C}_{32} \mathrm{H}_{66}$ and antioxidant molecules such as BHT, the apparent diffusion coefficients into either corn oil, triglycerides or ethanol are near or greater than $10^{-11} \mathrm{~cm}^{2} \mathrm{~s}^{-1}$ above $30^{\circ} \mathrm{C}$. The influence of solvent, temperature, and crystallinity on the diffusion coefficients of BHT in poiyethylene have been summarized earlier in this report. Therefore, for polyolefins, the diffusion limited case exists for these migrants and solvents only at a storage temperature lower than ambient, or at a much shorter storage time (a 10-fold reduction in the amount migrated requires a 100-fold reduction in time), or for a very thick container. The diffusion 1 imited case may also exist for extraction with poor solvents for the migrant. However, in the case with poor solvents, the solubility limit or partition limit requirements may often suffice. 
Fig. VII-1. A Simplified Additive Migration Decision Flow Chart

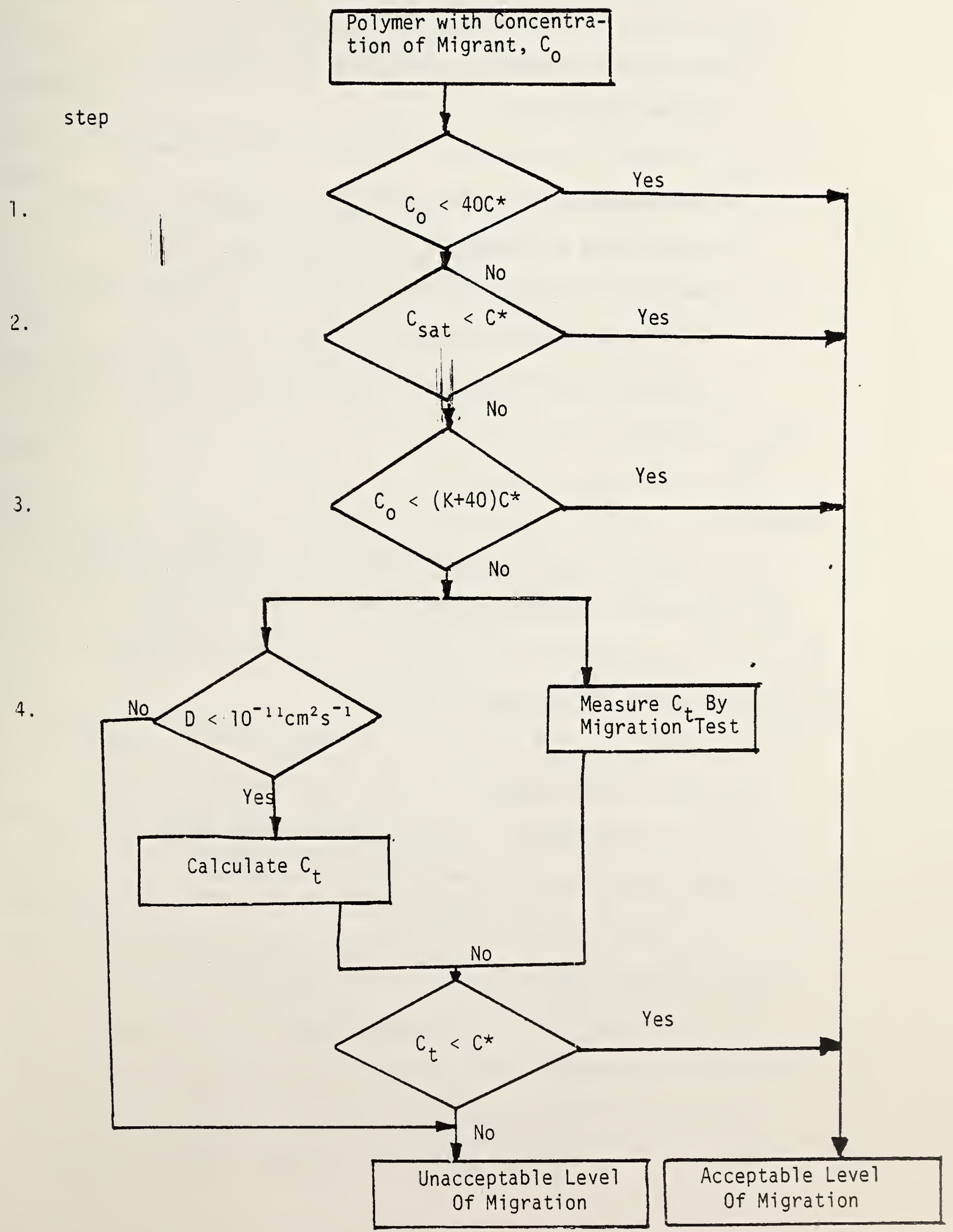


Figure VII-1. (Continued)

Glossary:

$C_{0}$ - Original concentration of migrant in polymer.

$C^{*}$ - Critical or acceptable concentration of migrant in food or solvent of concern.

$C_{\text {sat }}$ - Solubility of migrant in food or solvent of concern.

K - Distribution or partition coefficient of migrant in polymer and in food or solvent at equilibrium, $C_{\text {poly }} / C_{\text {solv }}$.

D - Apparent diffusion coefficient of migrant in polymer chain by, food or solvent of concern.

$C_{t}$ - Concentration of migrant in food or solvent after contact time t.

Notes:

1. This flow chart is based upon the assumption of a volume ratio of packaging material/food of $1: 40$ or $10 \mathrm{~g}$ of food are in contact with $1 \mathrm{in}^{2}$ or $6.45 \mathrm{~cm}^{2}$ of packing material. Consult text for other conditions.

2. Along the decision or branching points, move to the next step if the parameter in the particular step is unknown or not determined, the particular step may be skipped and the criteria of the next step will be tested. 


\section{Simulating Solvents}

Within the limited temperature range of food utilization and st age, solvent is the single most important parameter effecting both the amount of migrant extractable and the diffusion coefficient of the migrant, acting through its solubilities in and its swelling action upon the polymer.

By correlating diffusion coefficients in other solvents to that in one of the solvents, e.g. ethanol, while keeping all other parameters constant, it is possible to reduce the large spraad of diffusion coefficients of the order of $10^{8}$ to within a factor of 5 or less.

For partitioning or low solubilit.y solvents, the correlations depend not only on the solvent but also on the migrant and/or the polymer. However, for solvents having moderate solubilities toward the migrant,

- the correlation seems to be independent of migrant, polymer or temperature.

The most significant correlations are those found for pure triglycerides and ethanol as food oil simulants and that for the accelerating solvent, n-heptane. As shown in Figure VIII-I, the diffusion coefficients of migrants ( $n$-octadecane, $n$-dotriacontane or BHT) moving from polyolefins (LPE, BPE, PP) or E-VA copolymers into either tributyrin, trioctanoin or synthetic triglyceride mixture $\mathrm{HB} 307$ at either 30 or $60^{\circ} \mathrm{C}$ are almost the same as the corresponding diffusion coefficient in corn oil. The diffusion coefficients in the pure triglycerides are slightly higher than that in corn oil with a ratio of $D$ (pure triglycerides)/D(corn oil) less than 1.5 over most of the range studied. Ethanol seems to behave almost identically to the lower members of pure trigiycerides in its extraction behavior as shown in Figure VIII-2. However the correlation for the data of ethanol extractions shows more scatter than that for triglycerides within a factor of less than 3 or less. 
From the above observations, either ethanol or low members of pure triglycerides may be used successfully for most occasions as food $0 i 1$ simulants, regardless of dissimilarities in viscosities, solvation power for the migrants, and swelling power for the polymers.

The accelerating action of $n$-heptane over that of food oil has long been recognized. The amount extractable by food oil was sometimes considered to be $1 / 5$ of that by $n$-heptane. However we found that no constant factor will describe this accelerating action. Beside the differences in solubilities and in partition coefficents, the accelerating power of $n$-heptane is clearly demonstrated in Figure VIII-3, where diffusion coeficients in $n$-heptane and in triglycerides (including corn oil) are plotted against those in ethanol for identical test samples and conditions. Regardless of polymer, migrant and temperature, the accerating power is greater at low diffusion coefficients than that at high diffusion coefficients. While, at $D$ (ethanol) around $10^{-7} \mathrm{~cm}^{2} \mathrm{~s}^{-1}$, accelerating power is greater at low diffusion coefficients than that at 1000 at $D$ (ethanol) around $10^{-11}$ or $10^{-12} \mathrm{~cm}^{2} \mathrm{~s}^{-1}$.

The accelerating action of n-heptane compared to that of ethanol (and that of triglycerides), coupled with the differences in solubilities of oligomers of polyolefins, has dramatic effects on the total extractables of polyolefins. We, therefore, subjected large quantities (50 g) of the raw polymer stocks of SRM 1475 (LPE) and SRM 1476 (LPE) to extraction by $500 \mathrm{ml}$ of ethanol $\mathrm{n}$-heptane at $70{ }^{\circ} \mathrm{C}$ for 160 days with occasional shaking. By assigning a minimum diffusion coefficient of about $10^{-9} \mathrm{~cm}^{2} \mathrm{~s}^{-1}$ for $\mathrm{n}-\mathrm{C}_{32} \mathrm{H}_{66}$ and a pellet radius of $0.1 \mathrm{~cm}$, the effective time $T=D t / \ell^{2}$ is 1.4. Thus according to the generalized solution for the diffusion equation, the extraction should be at least $95 \%$ complete. The results of the 160-day extractions are listed in 
Table VIII-I. For each polymer, about 6 to 8 times as much of polymer fraction was extracted by $n$-heptane than by ethanol.

The extracts were then subjected to gel permeation or size exclusion chromatographic analys is by Roger C. Snyder of FDA to find out their molecular weight distributions, as summarized in Table VIII-2 and shown in Figure VIII-4 for ! inear polyethylene SRM 1475 extracts, respectively. The molecular weight scales were calibrated by means of analyzing five NBS polyethylene standard reference materials, three nalkanes $\left(c_{10}, C_{20}, C_{44}\right)$ and test mixtures containing known amounts of $n-$ alkanes from $C_{\tilde{\sigma}}$ to $C_{44}$. The molecular weight scale for branched polyethylene is only approximately correct, however it should be sufficient for comparison purposes.

The extracts from the linear polyethylene show rather normal symmetrical distributions with peaks of molecular weight centering at about 300 for ethanol and at about 800 for $n$-heptane extracts. Such results are expected from the practice of molecular weight fractionation by solvents of different solubility power. On the low molecular weight side, e.g., $n-\mathrm{C}_{18} \mathrm{H}_{38}$, there is a large enough solubility or even total miscibility of the oligomer fraction in both solvents, such that almost all of the low molecular weight fraction will be dissolved by the extraction processes, cf. labels $A$ in Figures VIII-4 and VIII-5. As molecular weight becomes higher, e.g., $n-C_{32}{ }^{H} 66$, the solubility in the solvent, especially in ethanol, decreases which causes a partitioning of the particular component between the polymer and the extracting solvent to occur, cf. Tabeis $B$ in Figures VIII-4 and VIII-5. The partitioning of $n-C_{32}{ }^{H} 66$ has been confirmed by extraction experiments with ethanol. The diffusion coefficients for larger molecules also drop significantly. Thus the combination of the effects of solubility, partitioning and diffusion created the shape of the molecular weight distribution curve beyond the peak molecular weight extracted. VIII-3 
A similar comparison for branched polyethylene is seen in Figure VIII-5. The skewed curves are most likely due to the incorrectness of the size versus molecular weight calibration which was based on $n-$ paraffins and linear polyethylene fractions. The peak of apparent molecular weight extracted is about 350 for ethanol and about 3200 for n-heptane extracts.

Therefore, $n$-heptane can not only accelerate the diffusion process but also remove high molecular species of oligomers that may be hardly present in ethanol or triglyceride extracts. 


\title{
Table VIII-1
}

\author{
Extraction of Polyethylenes \\ by solvents at $70^{\circ} \mathrm{C}$. for 160 days
}

\section{Fraction Extracted}

Solvent

LPE

SRM 1475

BPE

SRM 1476

Ethanol

0.0008

0.0023

n-Heptane

0.0047

0.018 


\section{Table VIII-2}

Molecular Weight Distributions of Ethanol and n-Heptane Extractions of Polyethylenes

$$
M_{n}: M_{w}
$$

Solvent

Ethano!

n-Heptane
LPE

SRM 1475

$250: 310$

$560: 1020$
BPE SRM 1476

$310: 410$

$620: 3000$ 


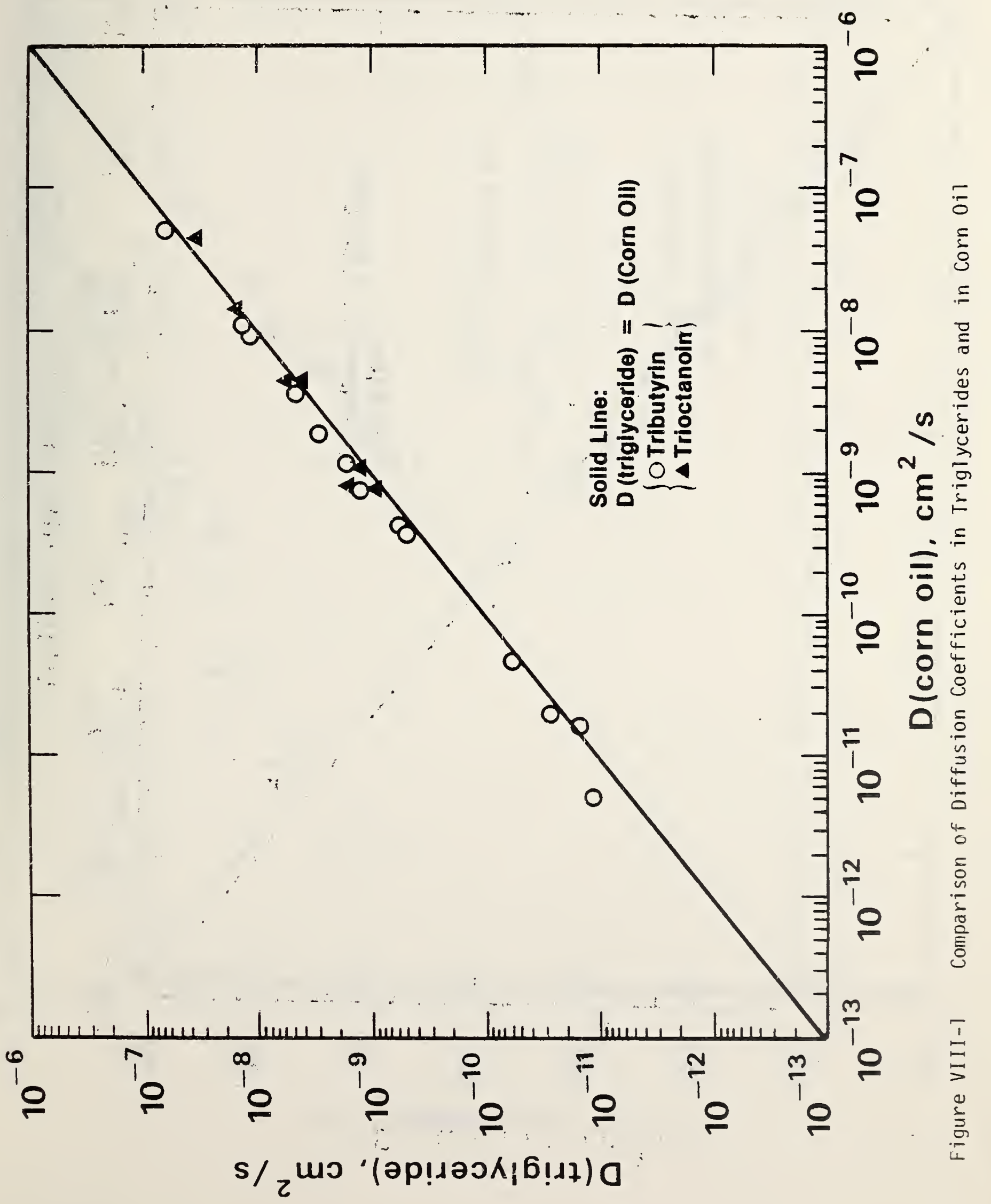




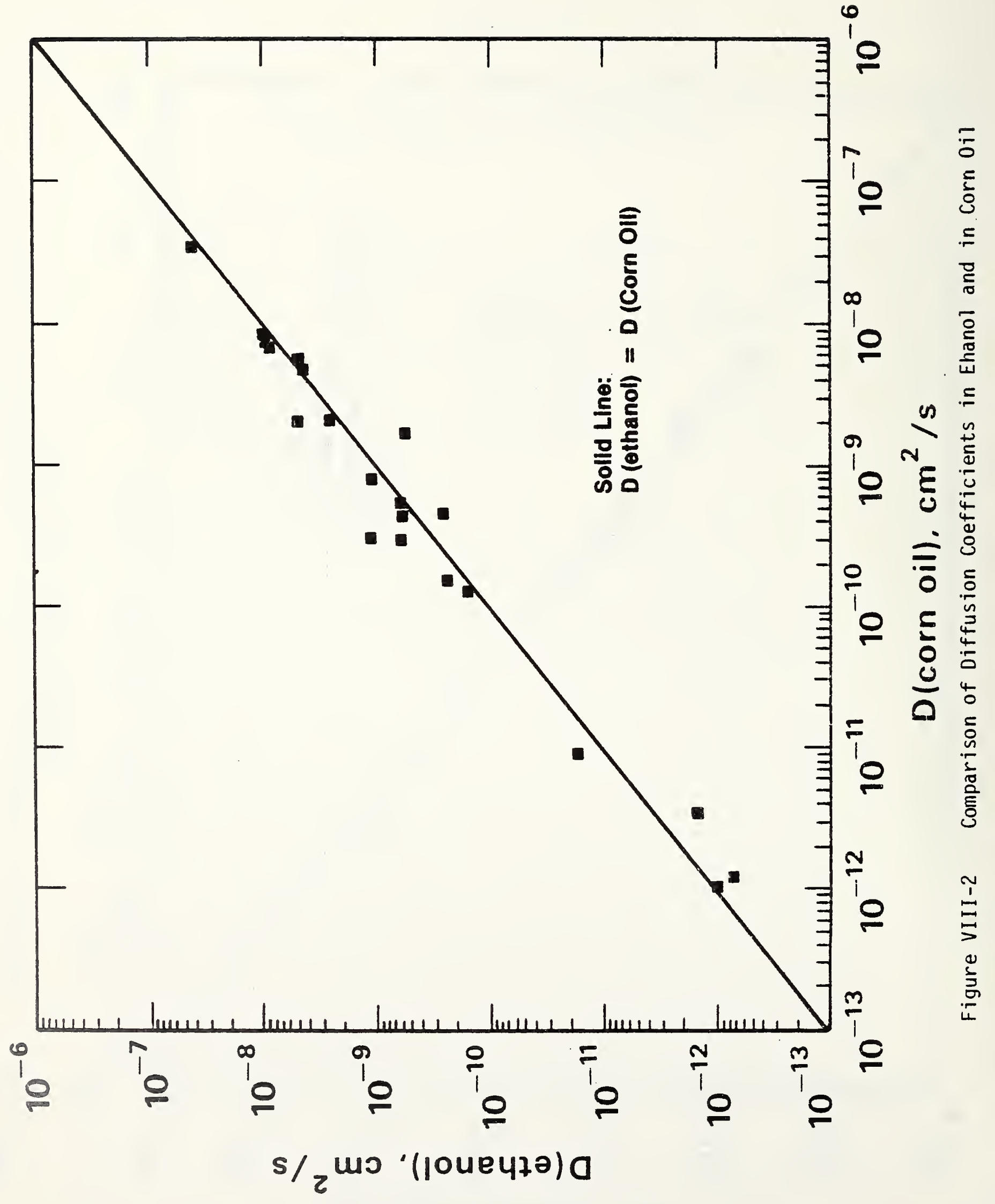




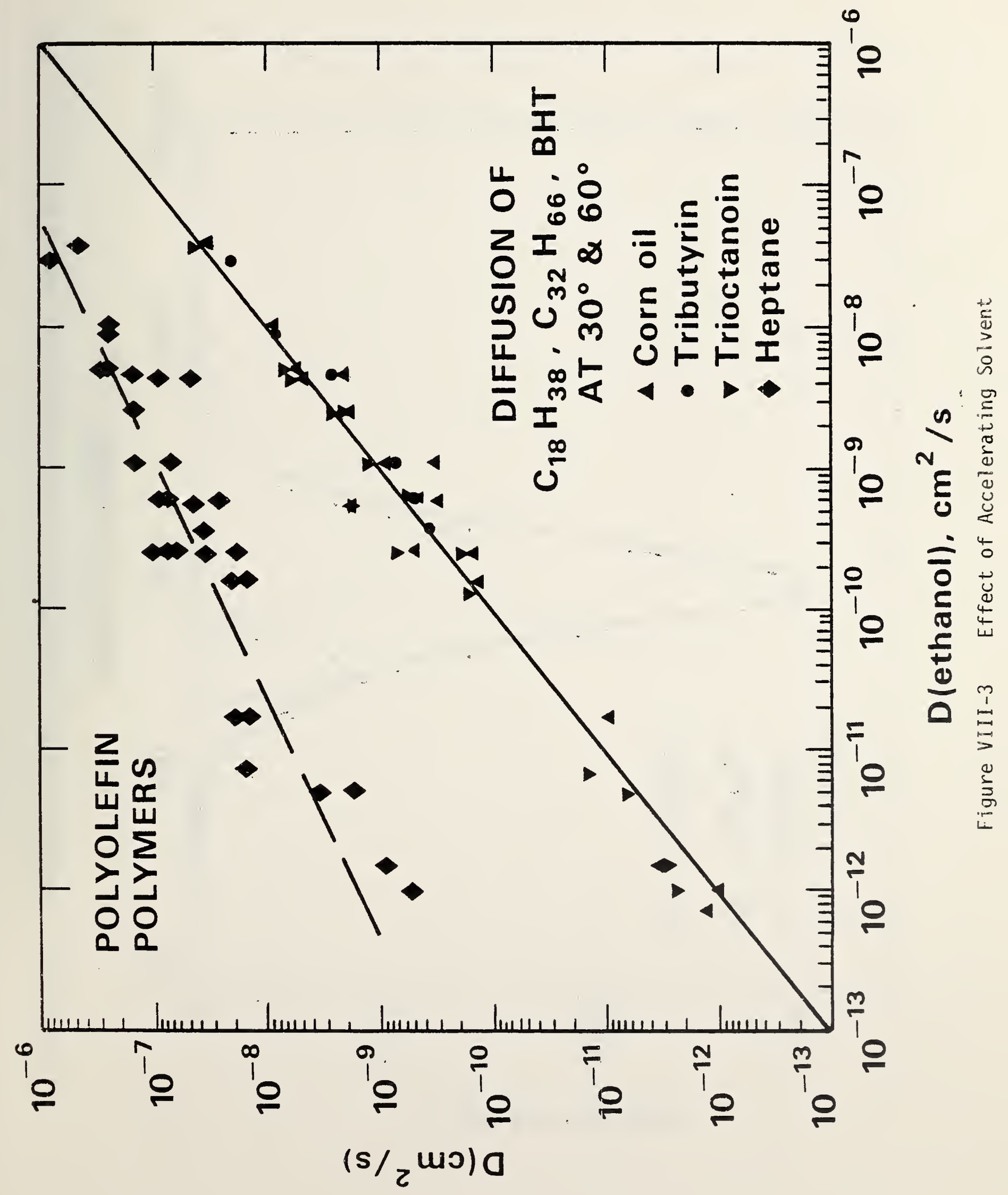




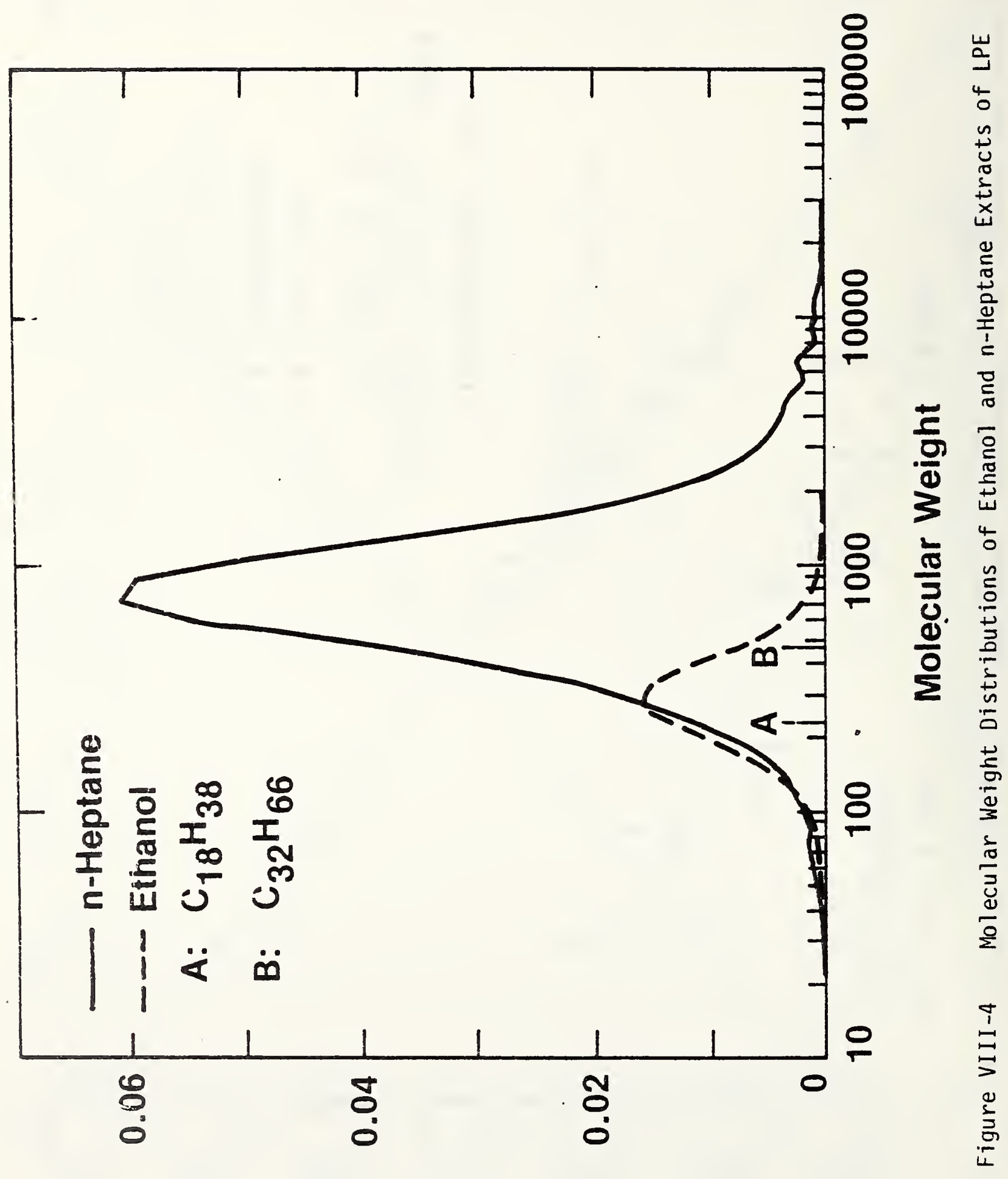

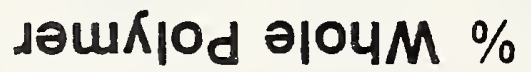




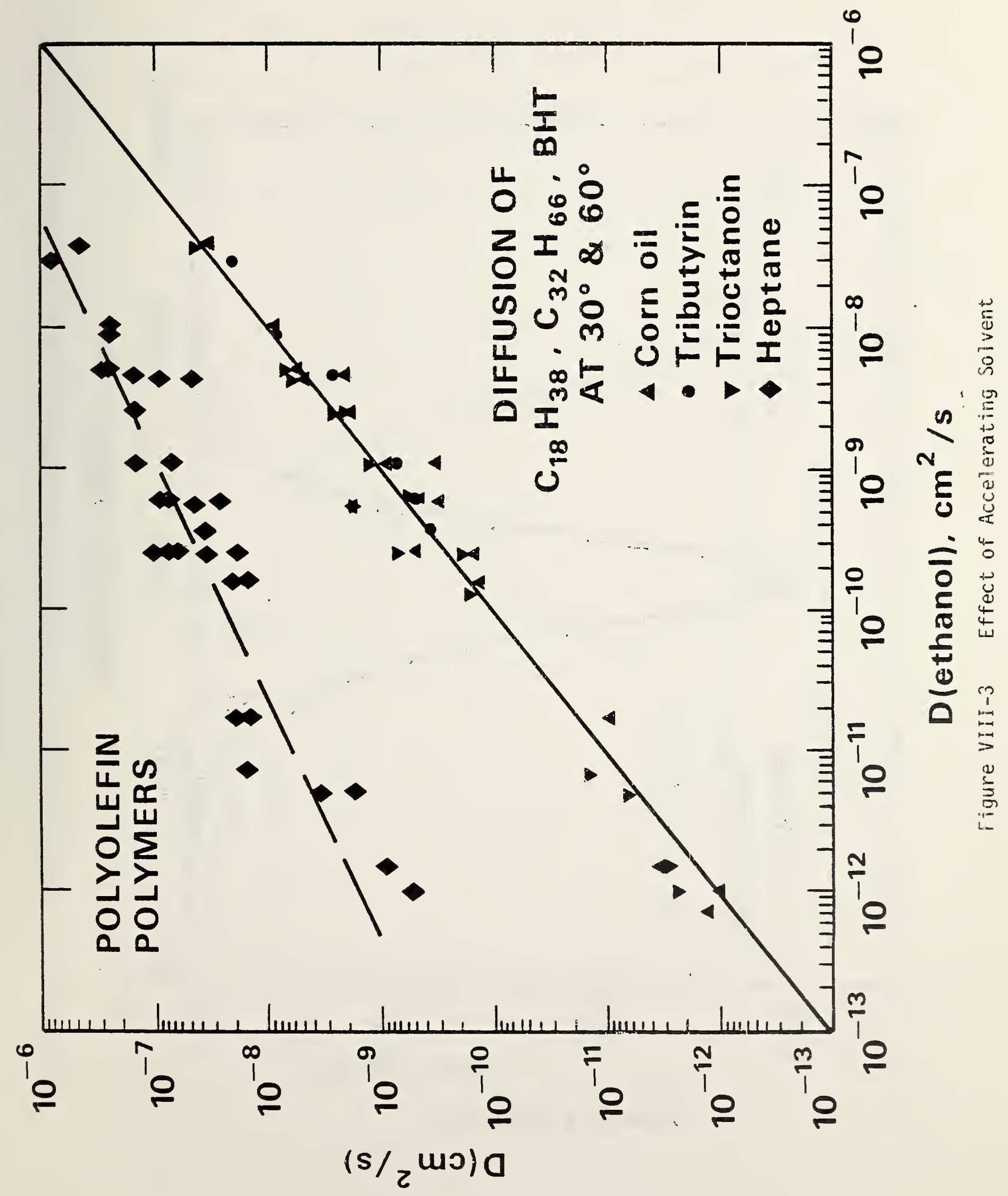




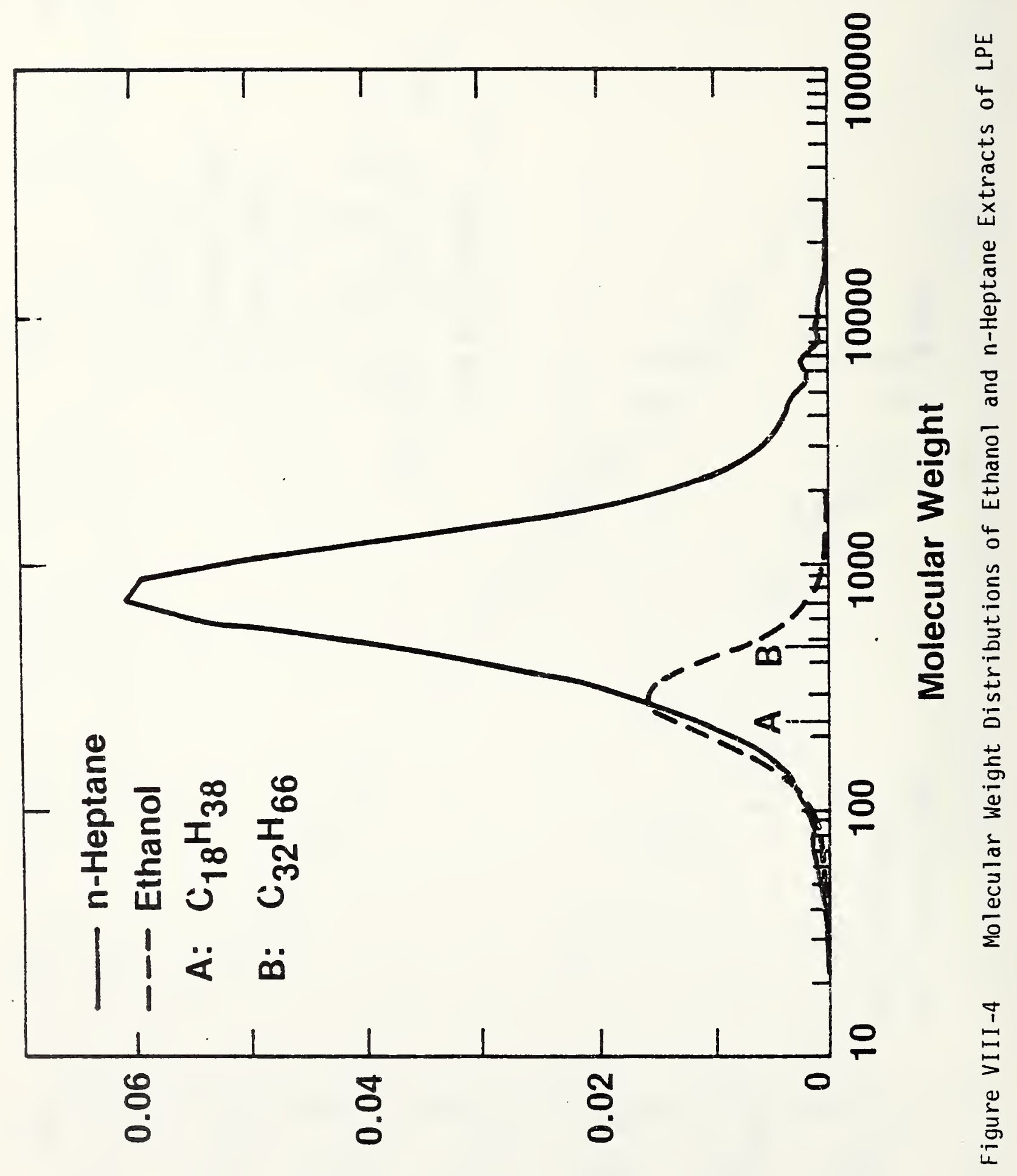

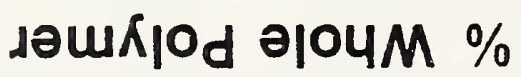




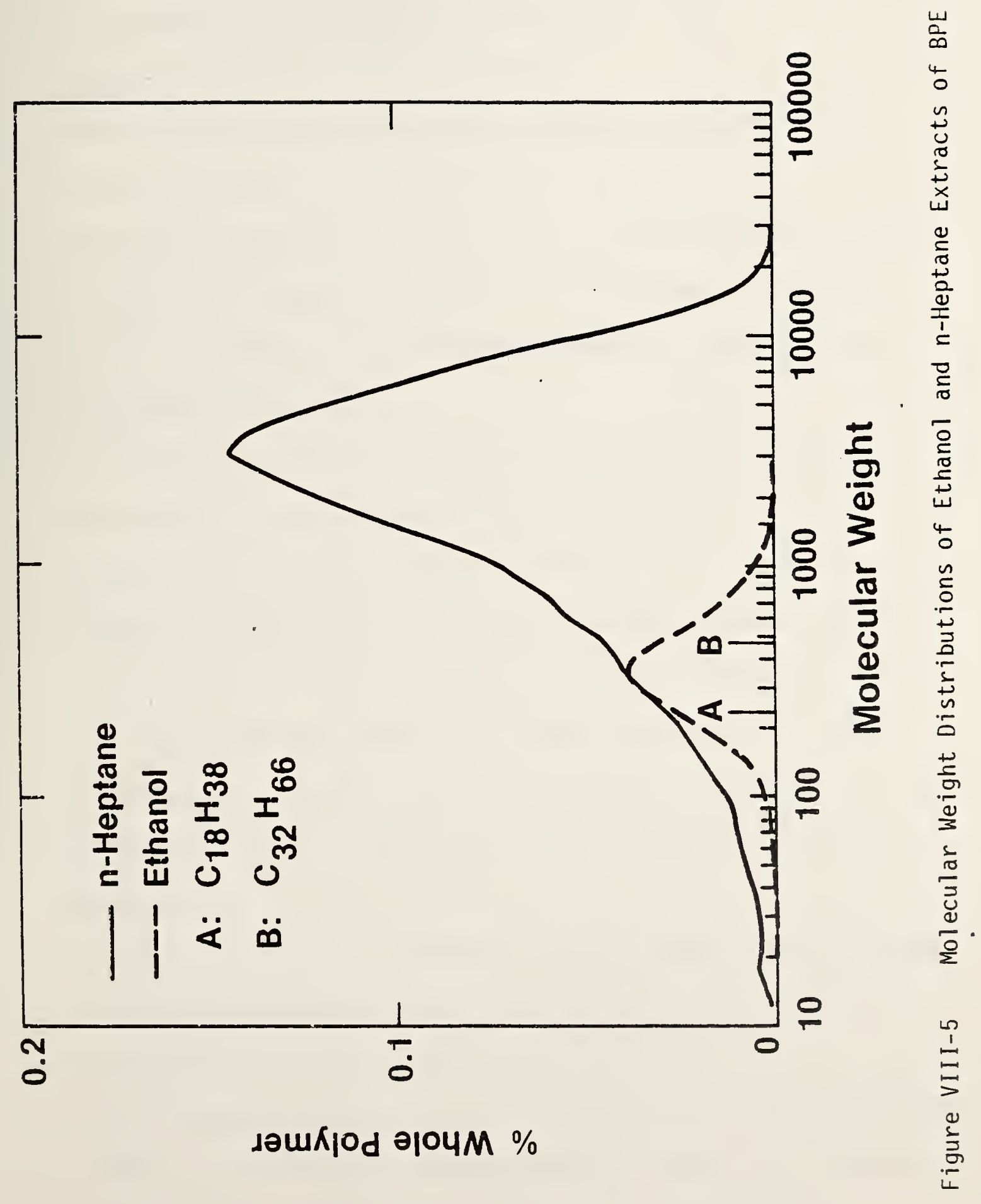




\section{Summary of Conclusions}

* Maximum amount of extractables depends on the original migrant concentration in the polymer, the solubilities and the partition of the migrant in solvent and in polymer.

* Migration behavior is mainiy Fickian.

* Diffusion coefficient may be increased by absorption of the solvent or swelling of the polymer by the solvent.

* Activation energies for the change of diffusion coeficient as a function of temperature generally lie between 40 and $200 \mathrm{~kJ}$ $\mathrm{mol}^{-1}$. In general, the activation energy is lower at higher diffusion coefficients.

* Lower members of pure triglycerides may be used to simulate the extraction behavior of corn oil in oligomers and antioxidants, such as BHT, from polyolefins.

* Anhydrous ethanol máy al so be considered as food-oil simulant.

* Accelerating action of n-heptane over that of food-oils or oil simulates is more pronounced at lower diffusion coefficients.

* n-Heptane will remove high molecular weight fractions of oligomers which may hardly be present in food oil or oil simulants.

* Diffusion coefficient increases with increased oligomer content.

* Increase of amorphous content in a polyolefin produces a corresponding change in diffusion coefficient.

* The following is the ranking of the importance of the parameters effecting the diffusion coefficient of a migrant from poiymer matrix into surrounding media:

Solvent > Temperature ( 1 imited range) $>$ Swelling

$>$ Migrant Concentration > Amorphous content. 


$$
\begin{gathered}
\text { NBSIR } 82-2472 \\
\text { Migration of Low Moleculer Weight Additives } \\
\text { in Polyolefins and Copolymers }
\end{gathered}
$$

X. Apperidix

Numerical Data of Migration of Low Molecular Weight Additives from Polyolefins and Copolymers

Experimental Run Designations--cf page II- 6 , Tables II-4 and II-5 


\section{.}




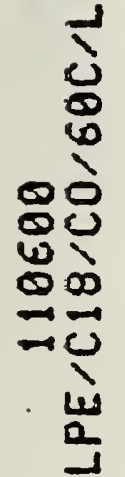

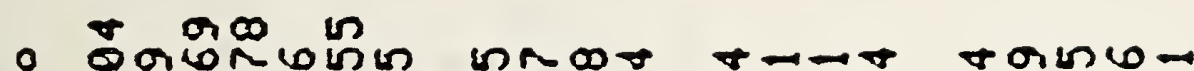

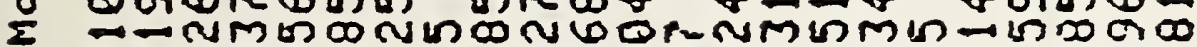
000000-1-num

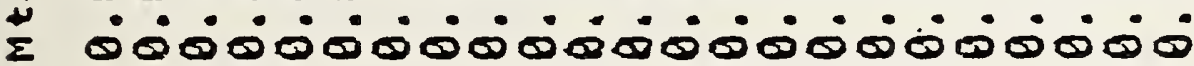

rmam

$1 \rightarrow m \in m g$

dongnen un an in

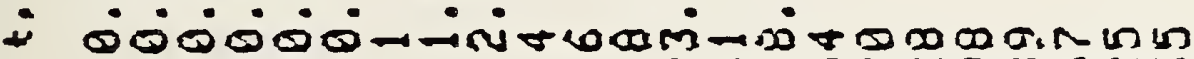

NMFUñon

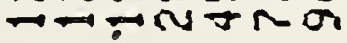

\footnotetext{
12060

-

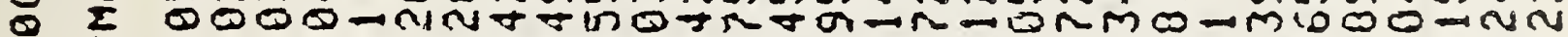

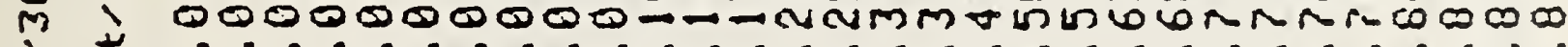
- = =

miv

mi

Qm

$\rightarrow$

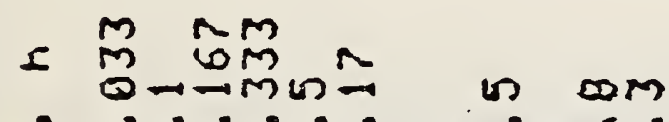

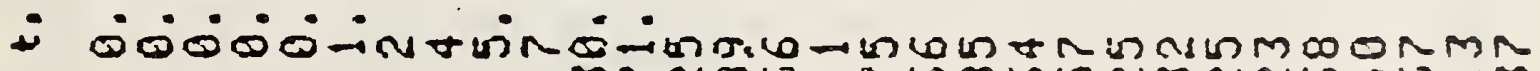

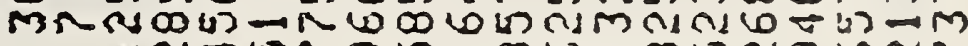

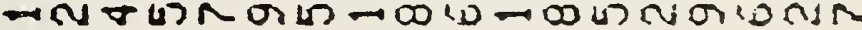

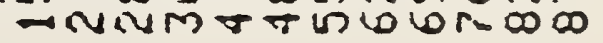




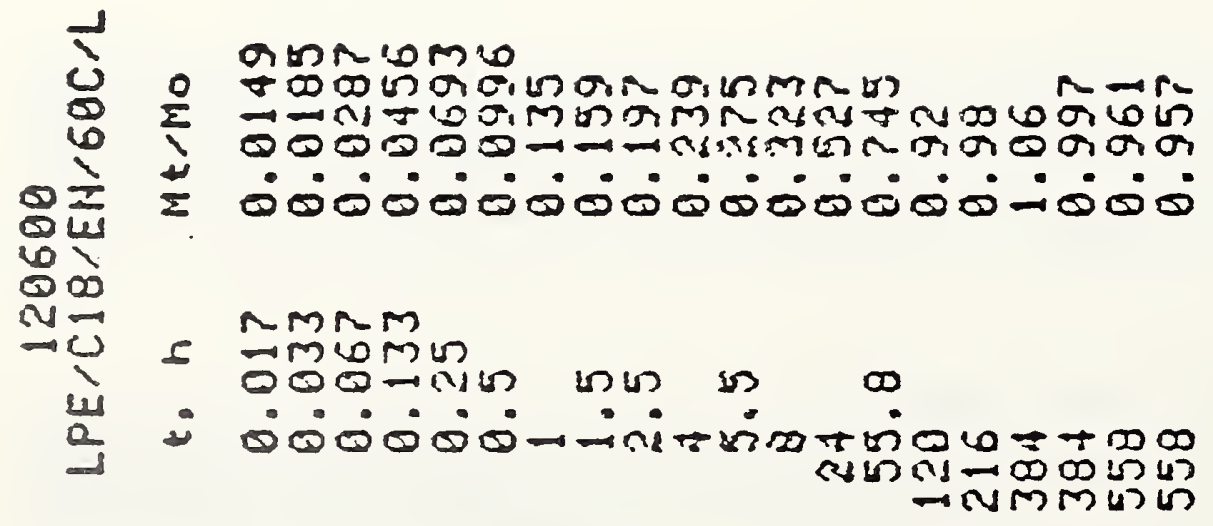




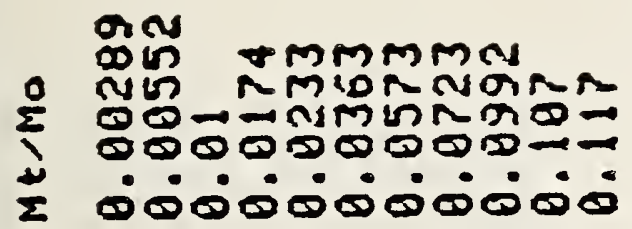

min

$\infty$.

$\infty$

nis

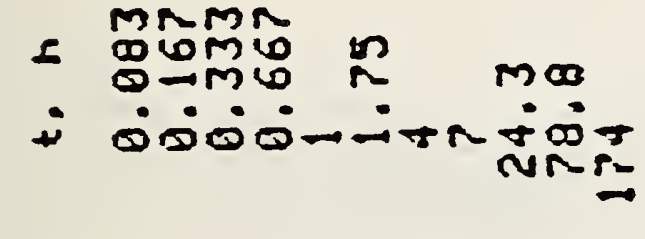

$\widehat{\Upsilon}$

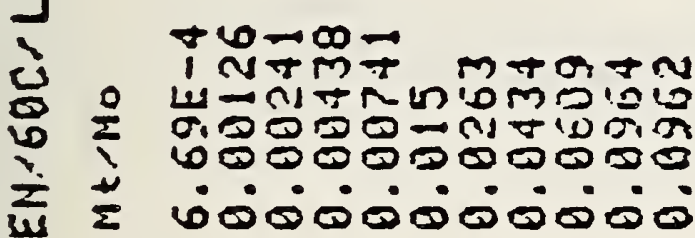

กงม

0

$10 \%$

ar

nmam

I $\rightarrow$ mbmin

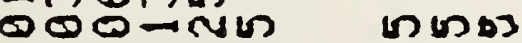

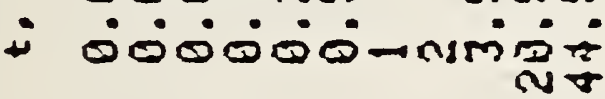

2

त) $T$

on

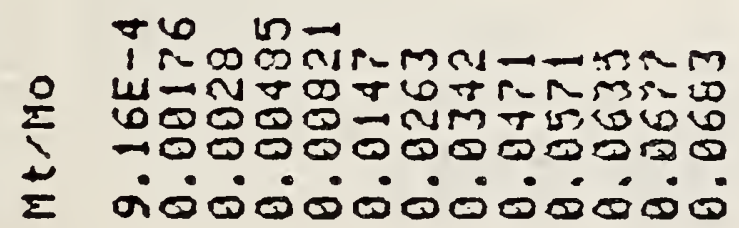

$\infty$

in

ni

NMNM

ᄃ $-m b \mathrm{~mm}$

DED-nu un un

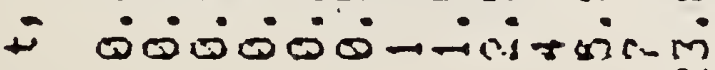




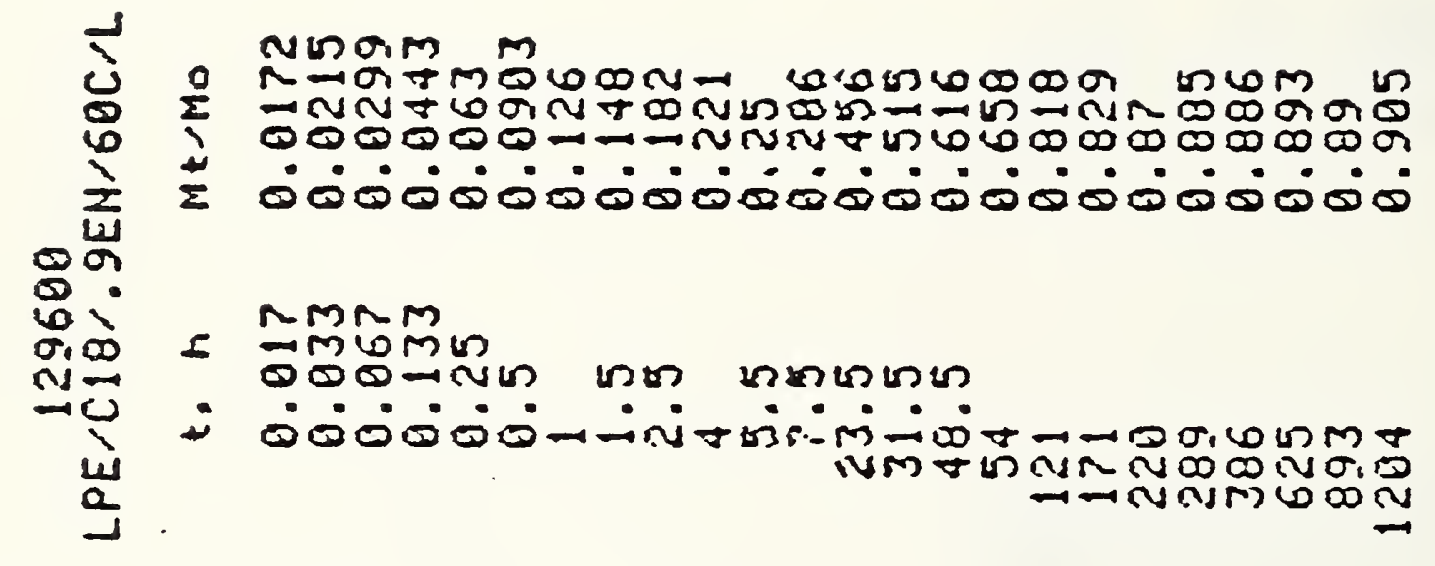

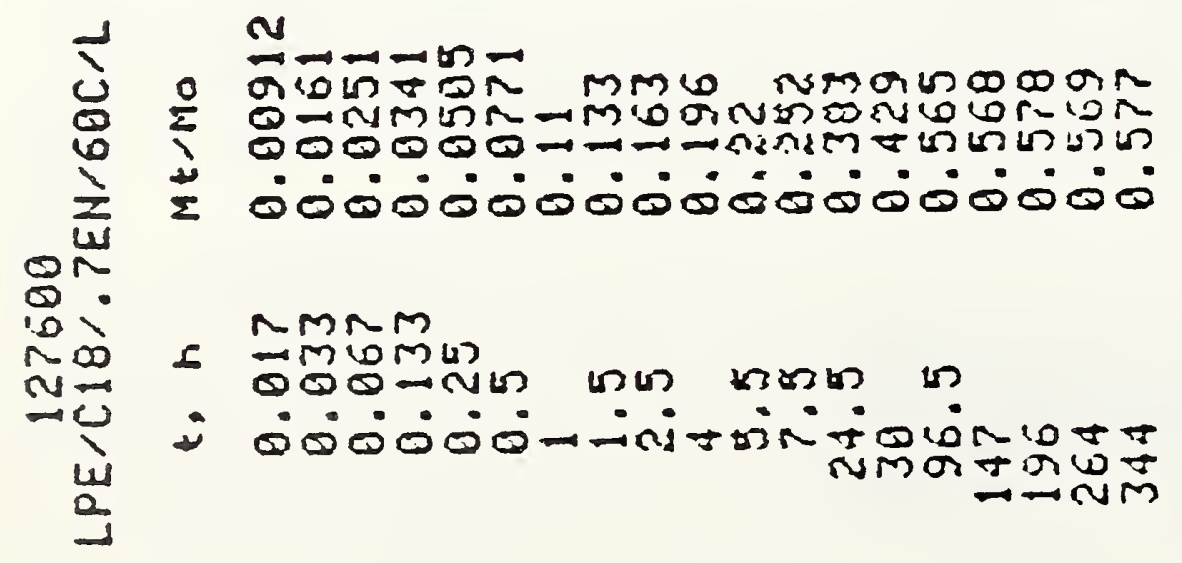


Common

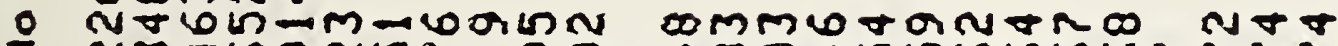
NM-

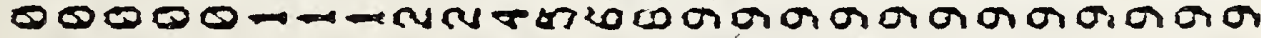

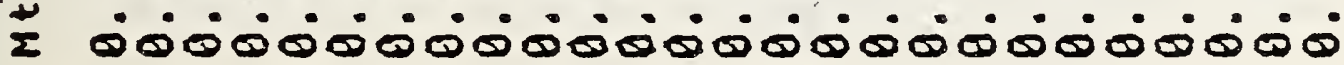

rmima

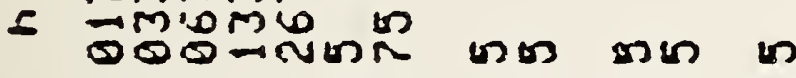

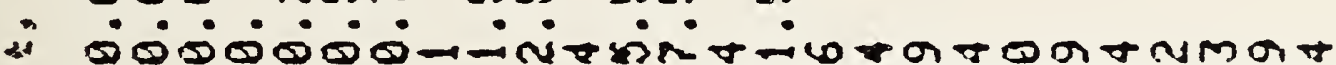
तMa $\rightarrow \pi N m+$ Un

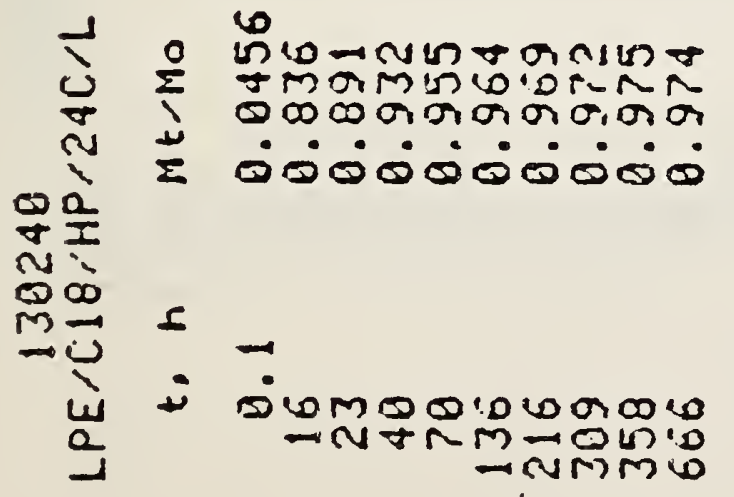

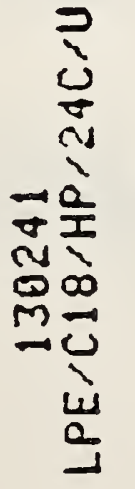

Nomr

- ogmngmandonarimat?

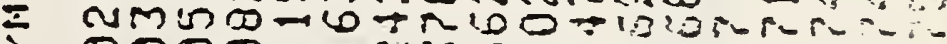

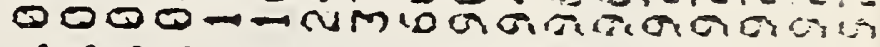

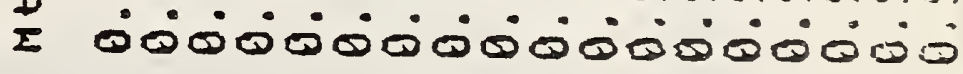

$\forall \frac{2}{1}$

त.

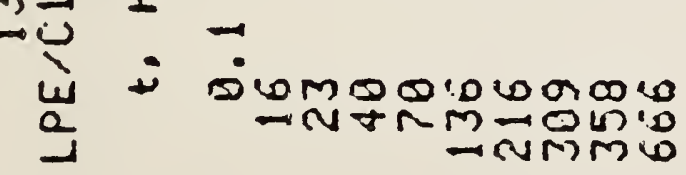

in

e nin in

$\infty 0-\pi$ in

- D்வ0்க்

Nin

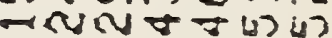



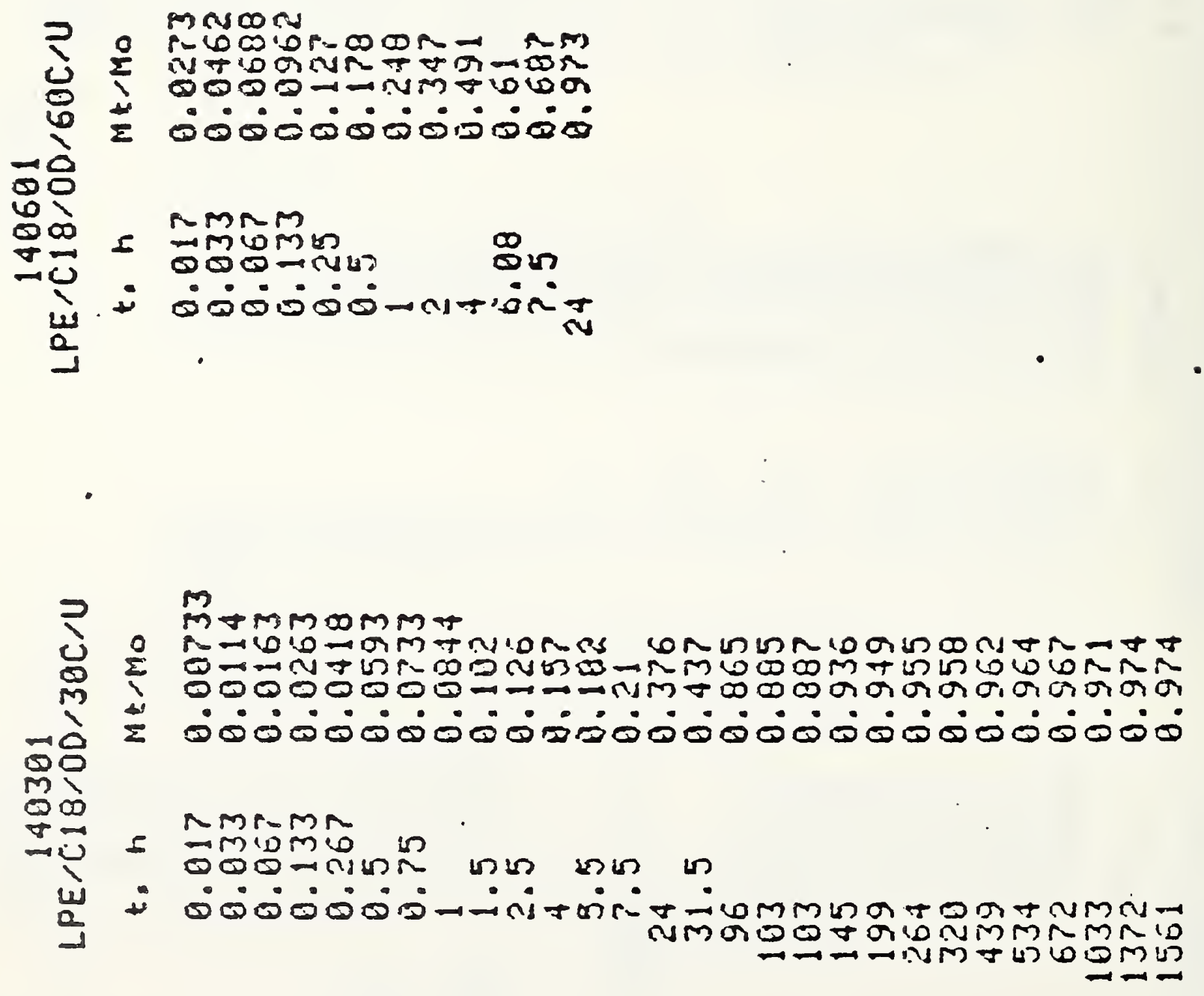


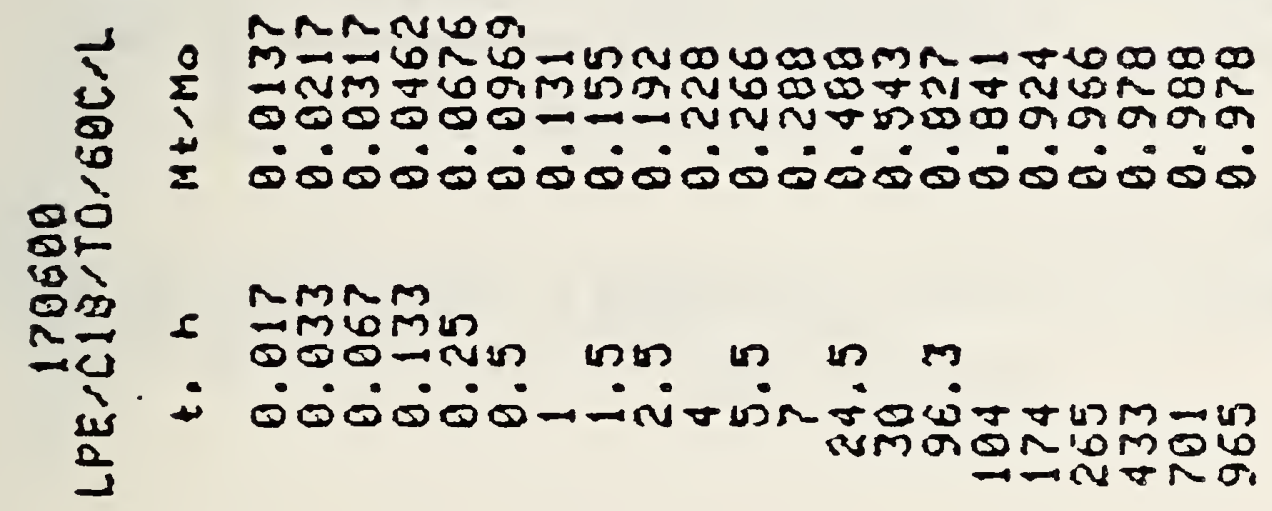

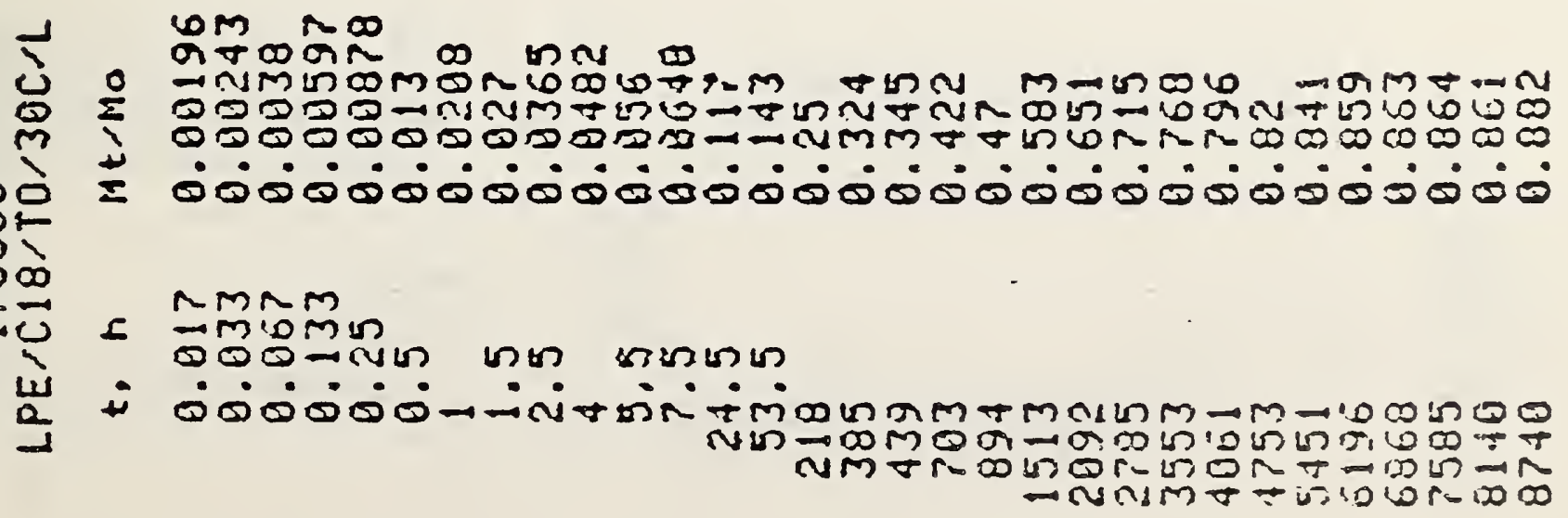



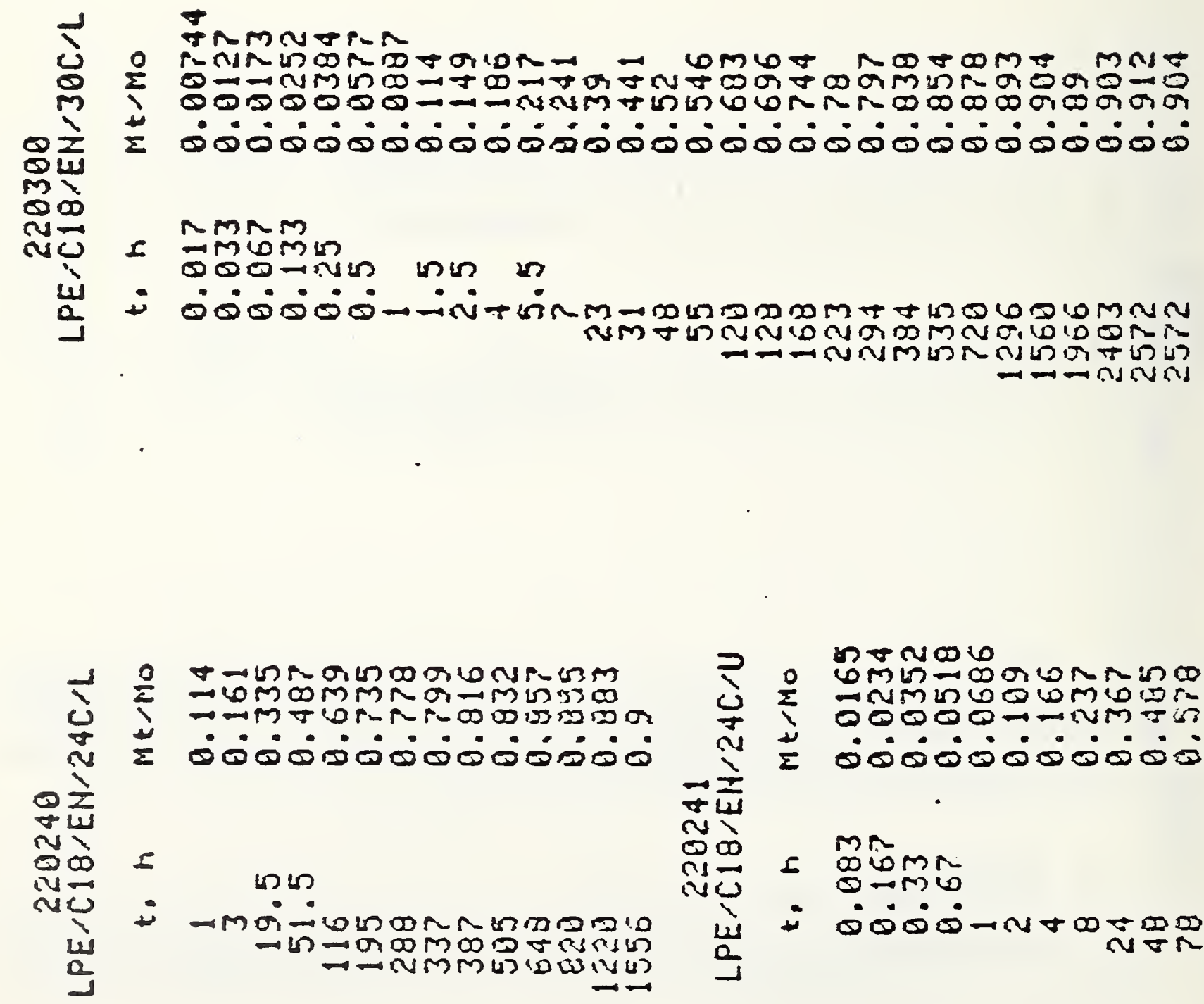
काष⿻

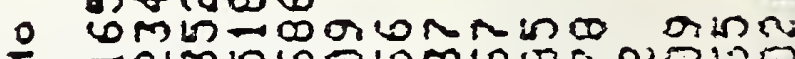

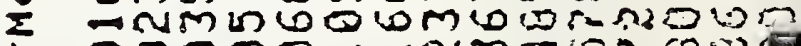

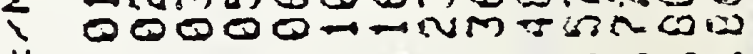

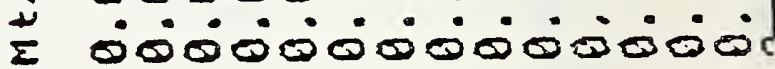




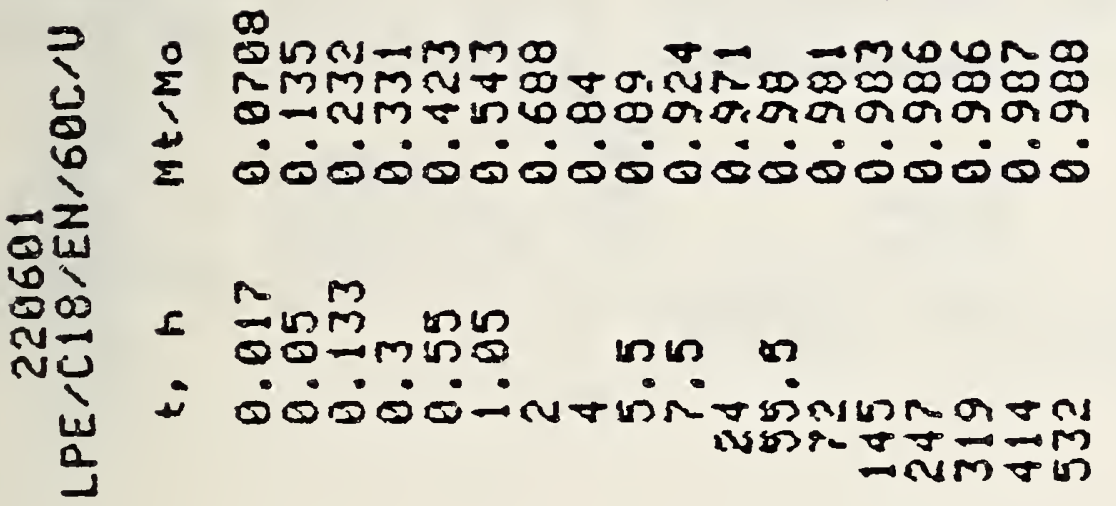

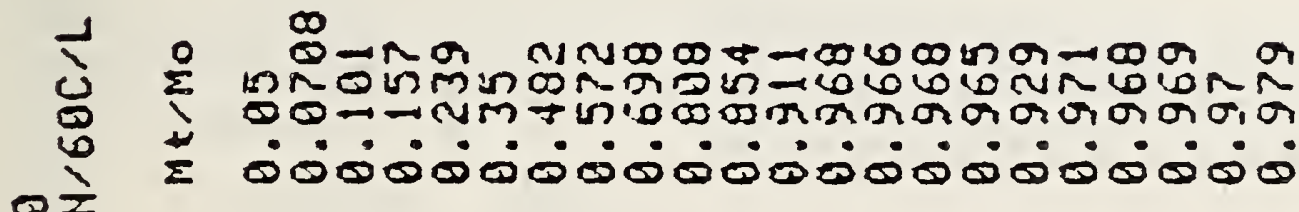



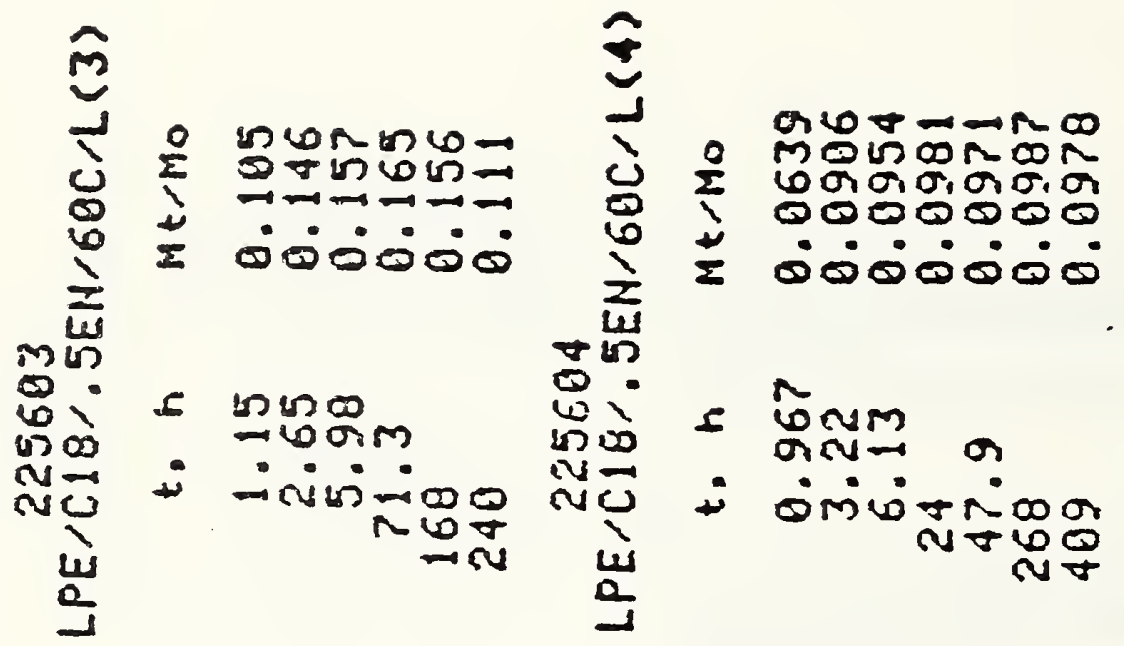

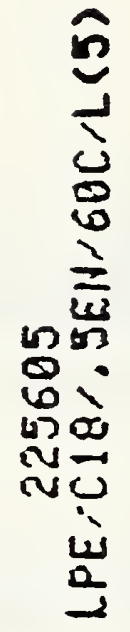

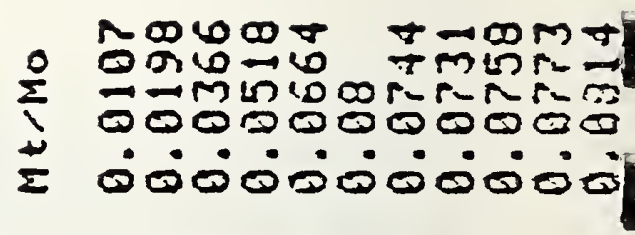

nm

-

in

- ?. oíara

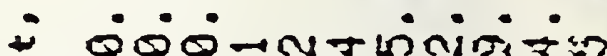

ruaró

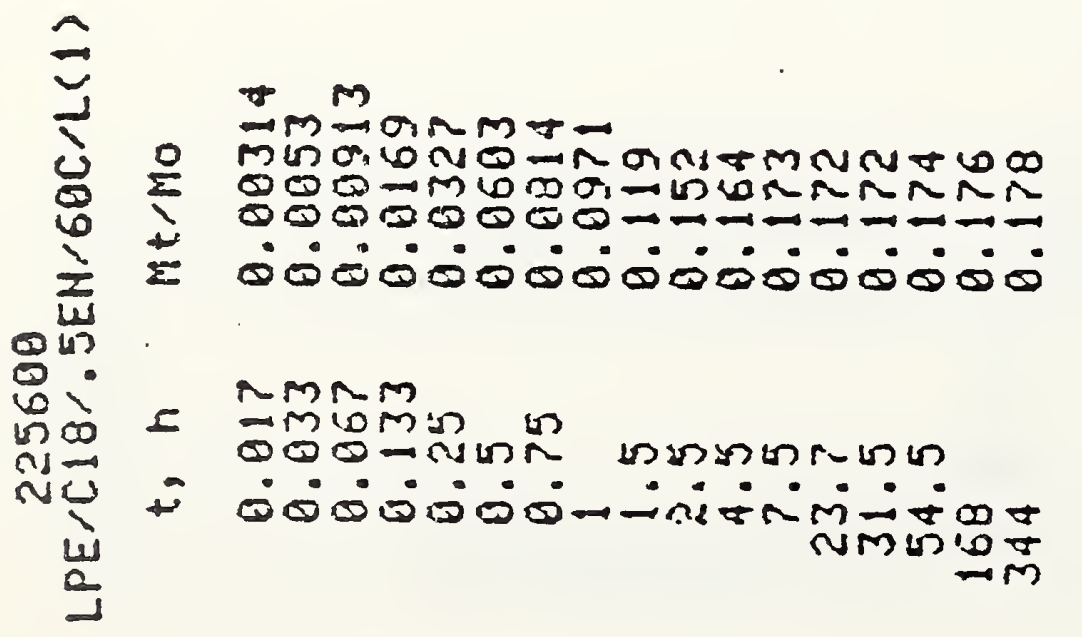

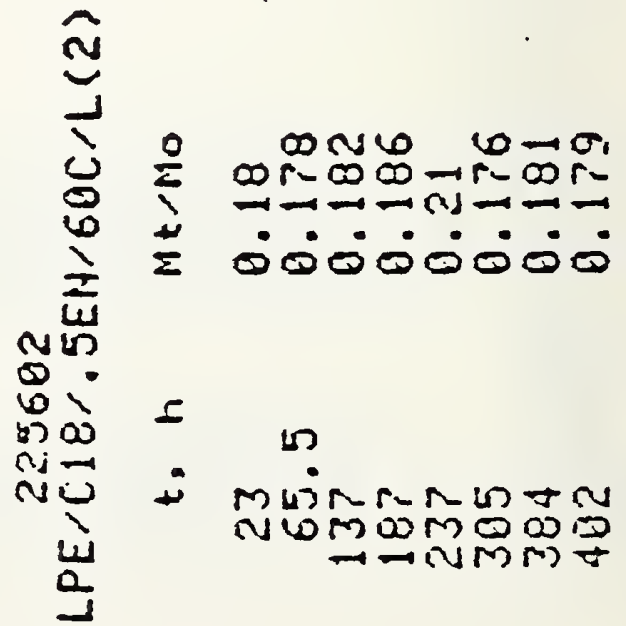




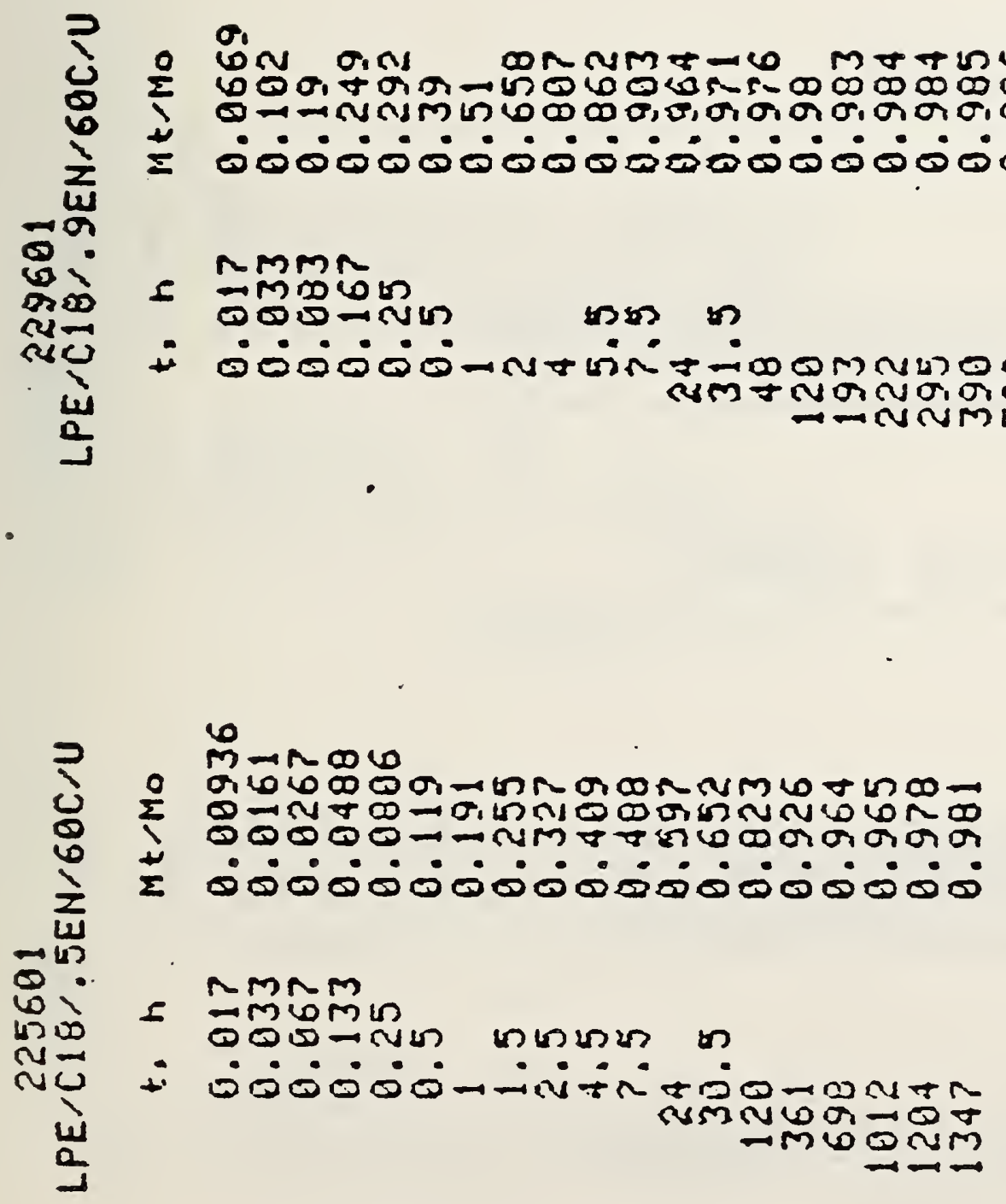



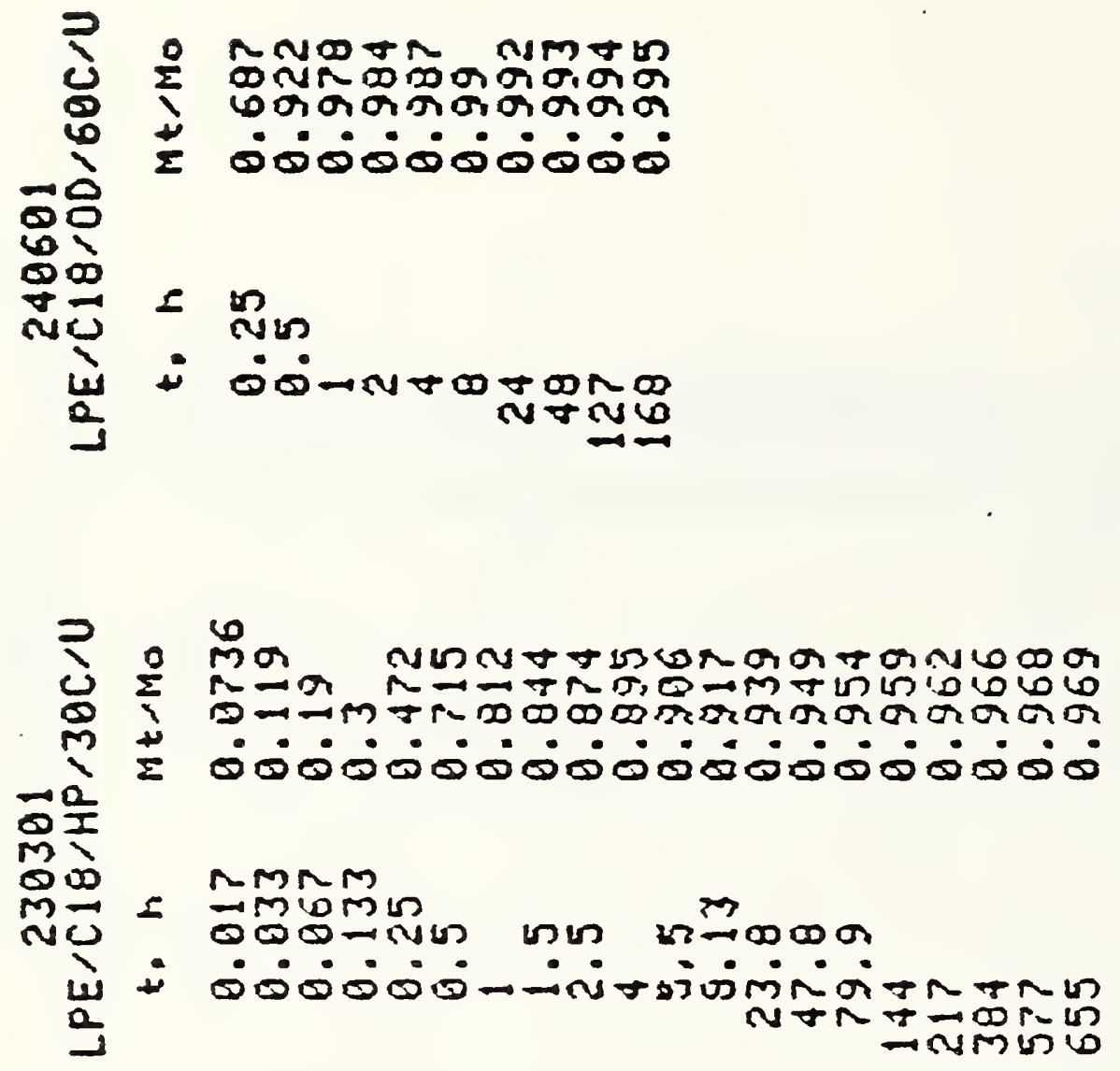

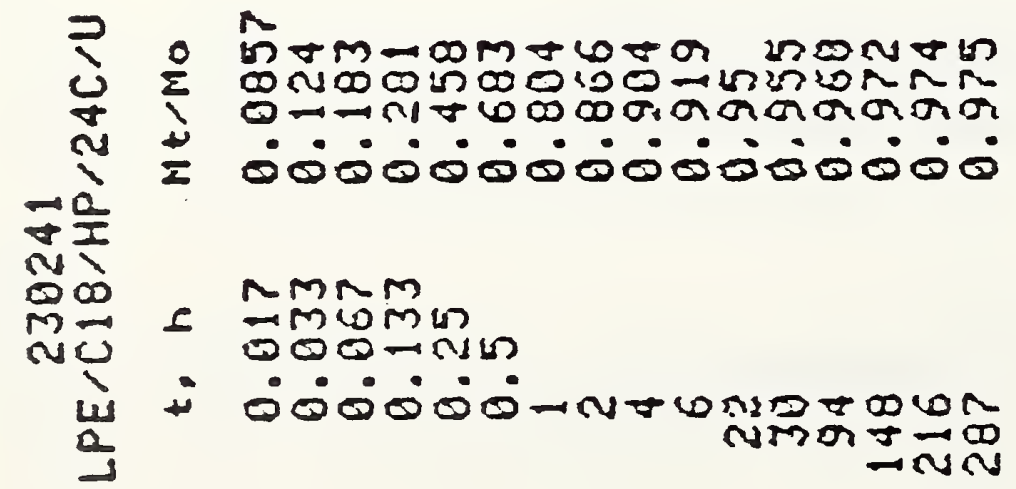




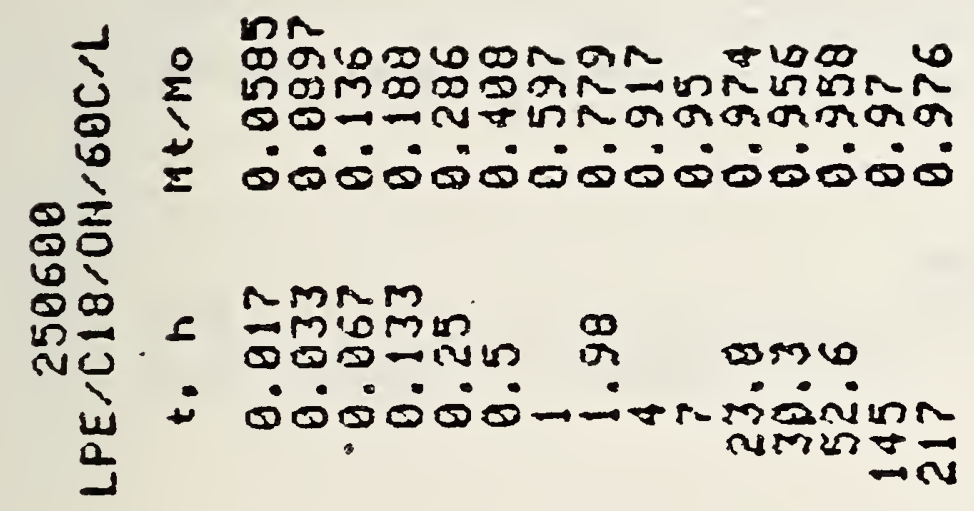

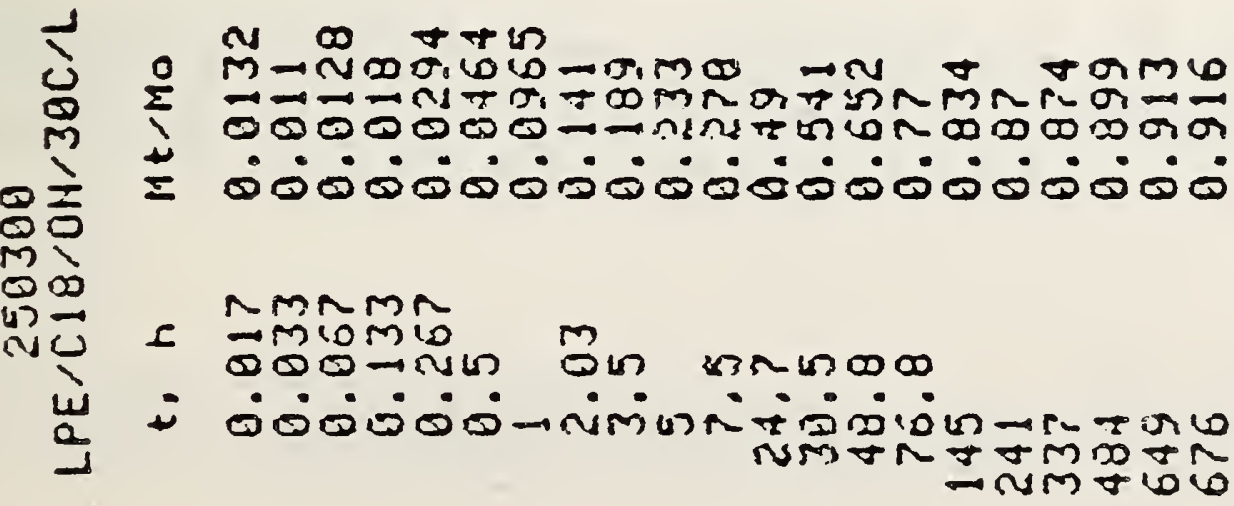




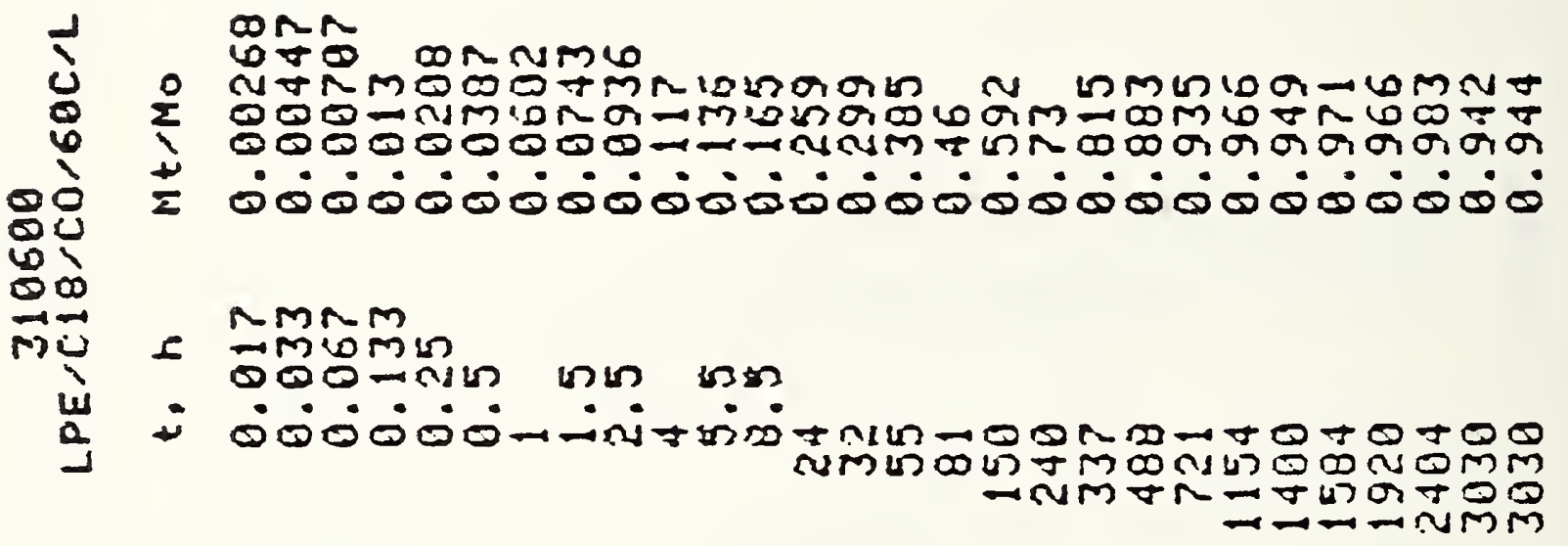

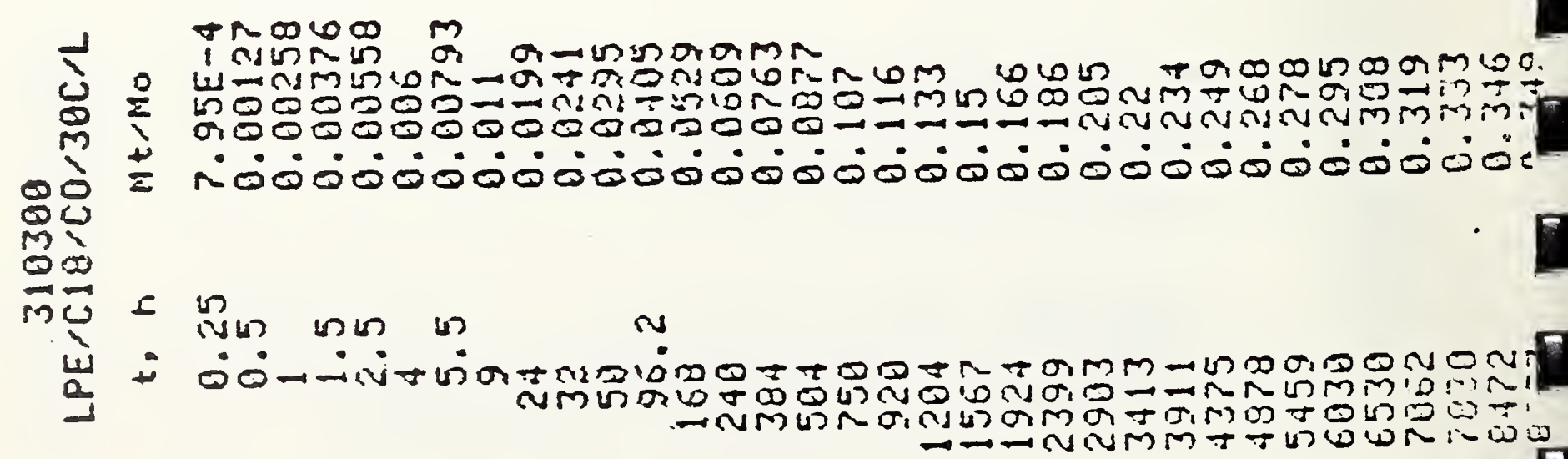




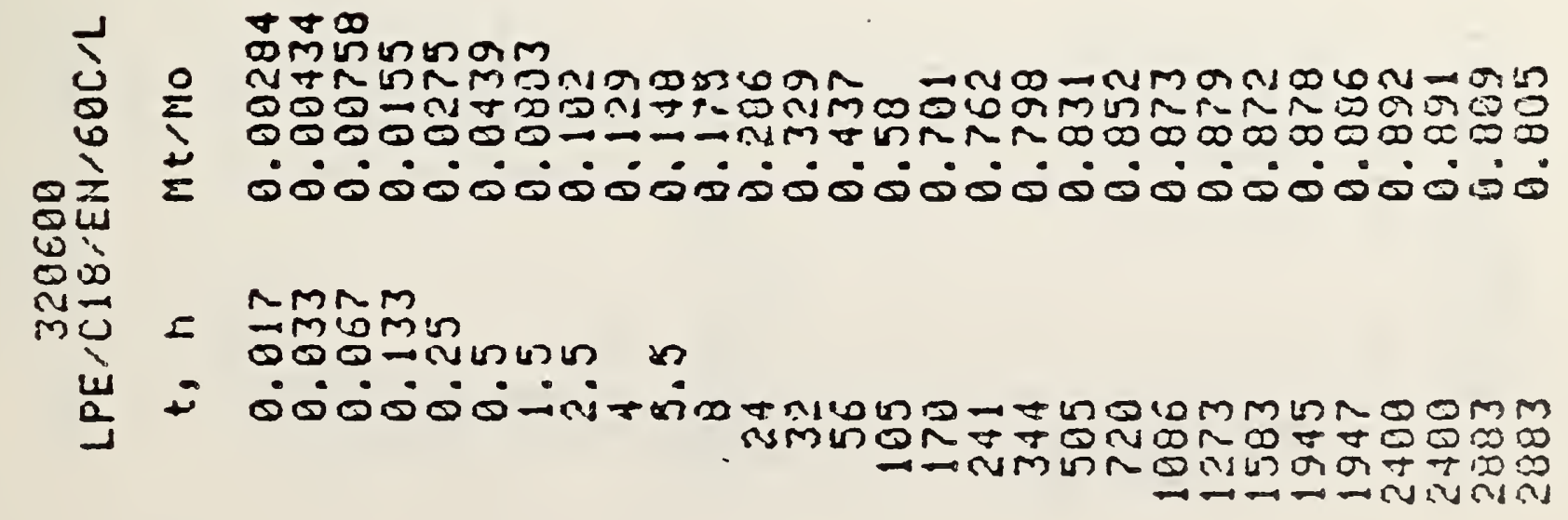



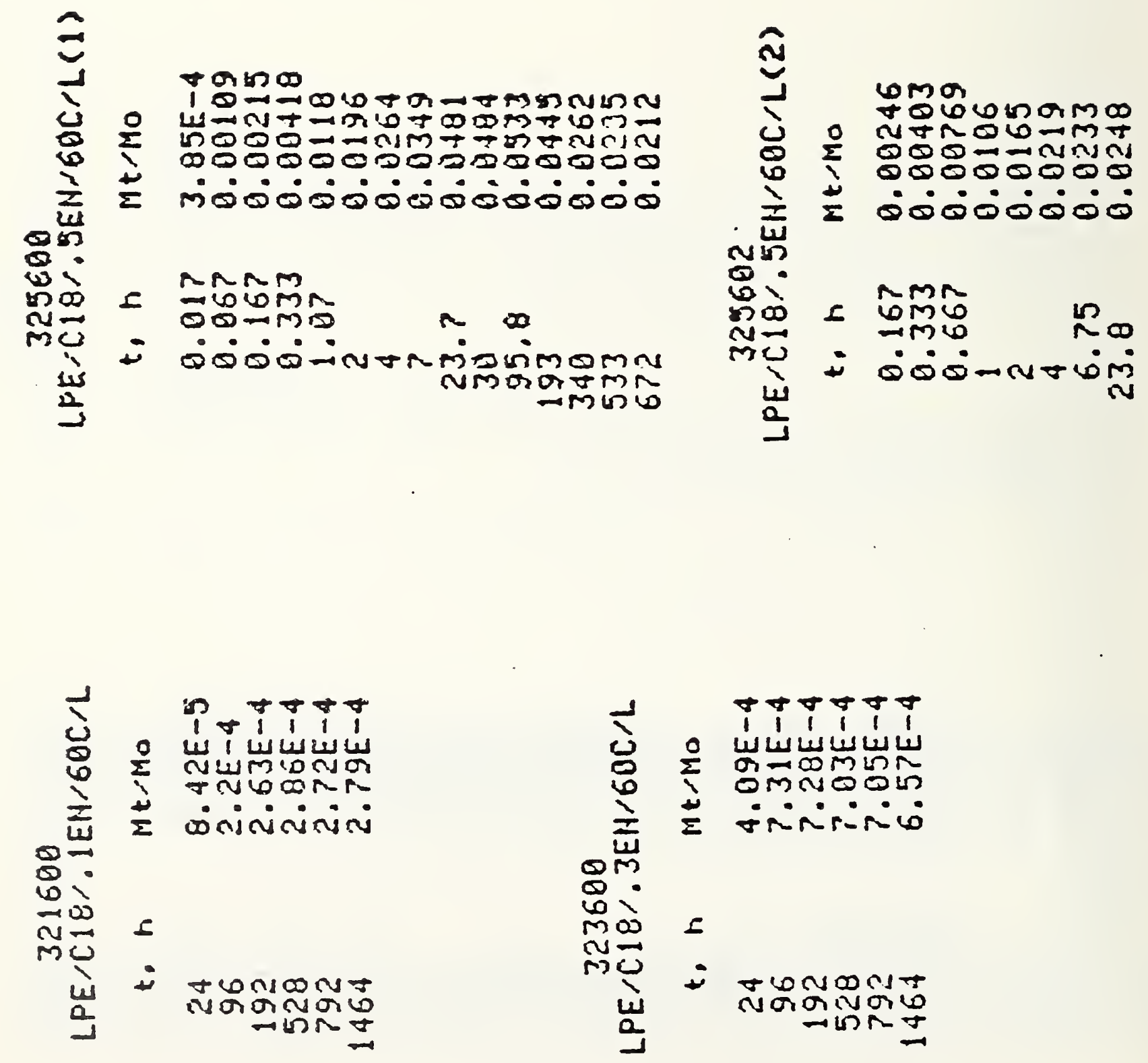


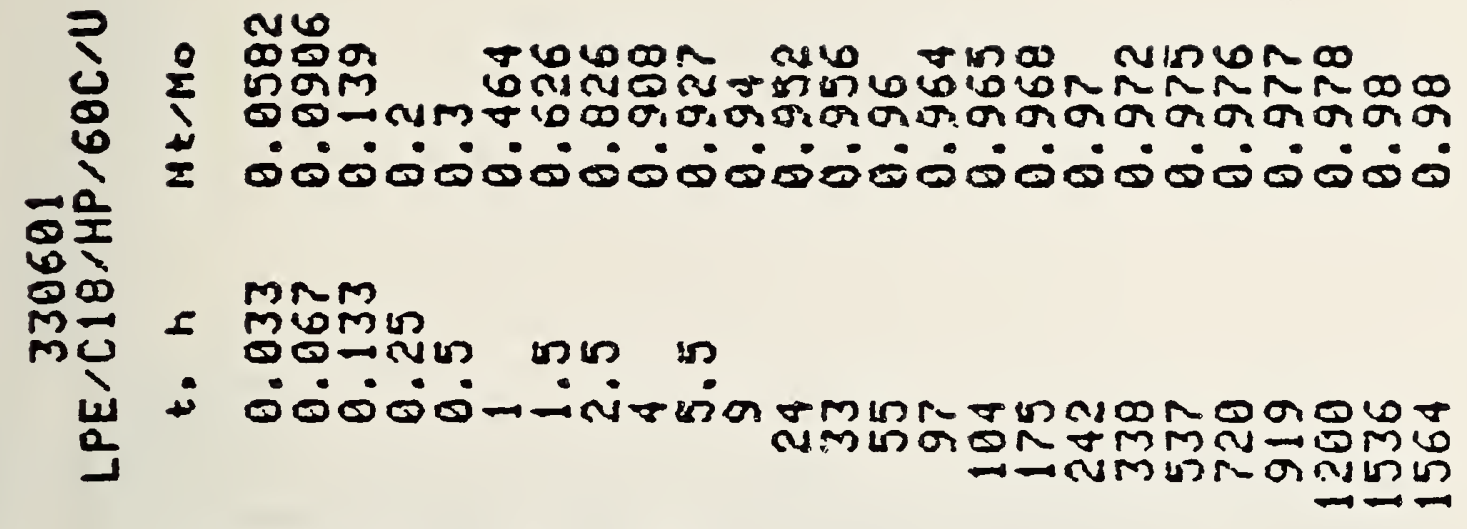

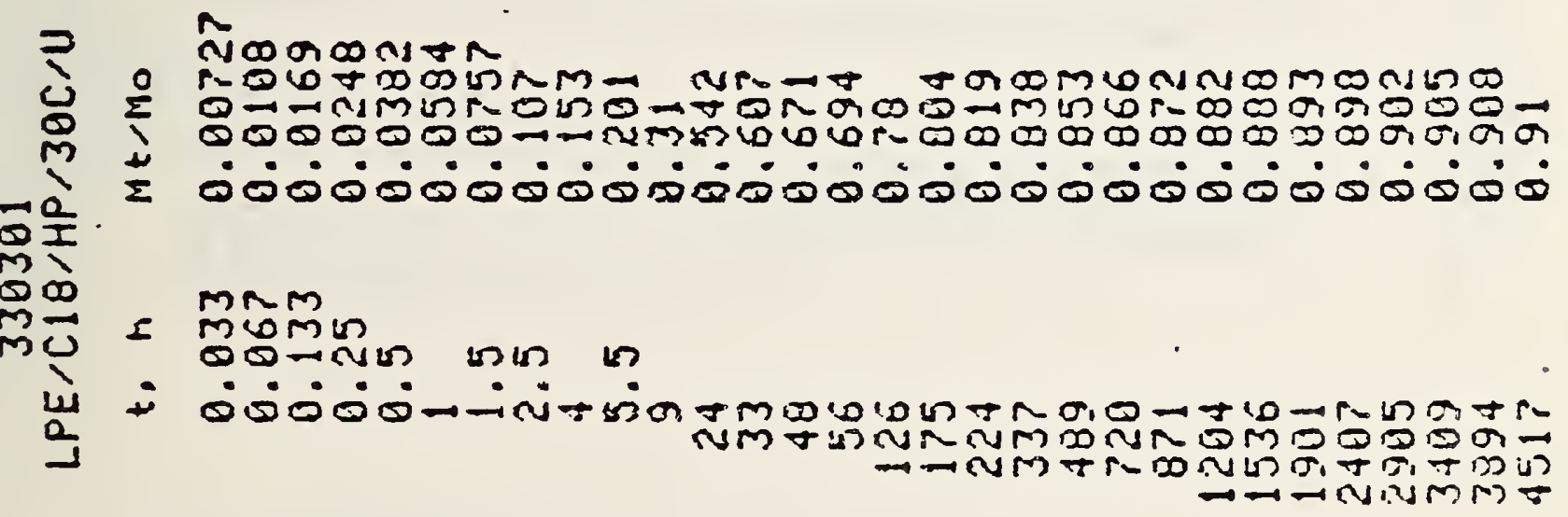

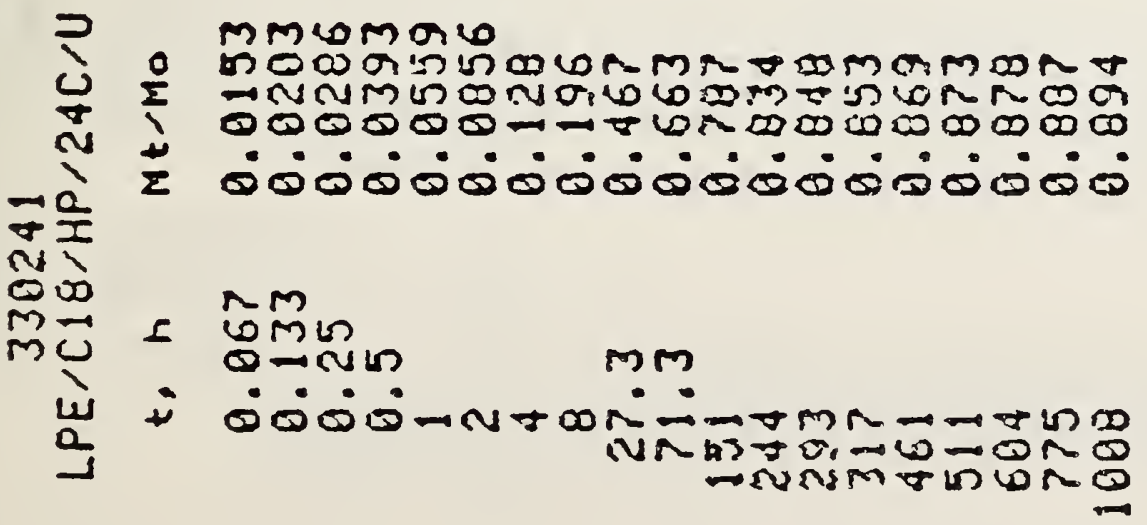




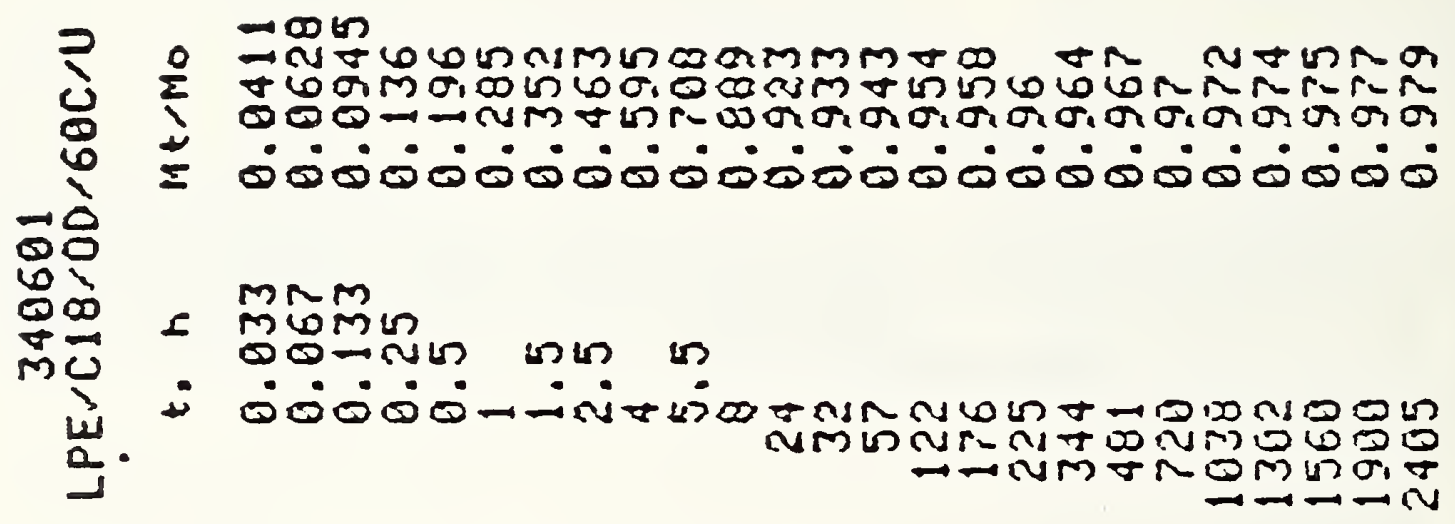

$\geqslant \quad M r \operatorname{mon} m+n$

¿ Tơngor

D $00 \div$ (n)

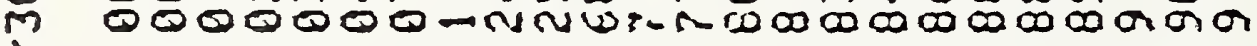

$\rightarrow 0$

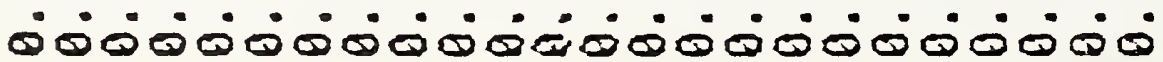

$m$

$\infty \infty$

\& $m R m$

mo owmi

$0 \rightarrow m \omega$

u.

a

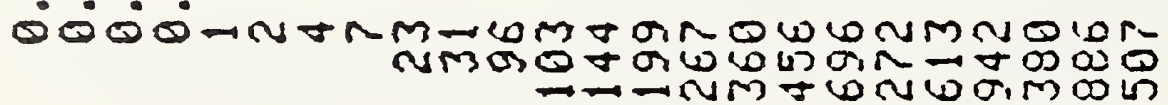
$-10 \mathrm{NM}$ 
$\operatorname{ara}$

$0 \pi \operatorname{Rn}=r$

OQD-n1

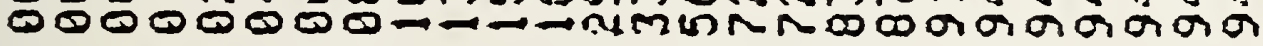

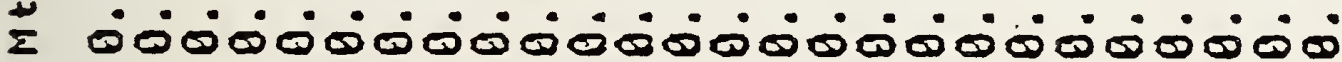

arm

W)

$0 \infty$

n

rmnm

I

0000 -nim nu n

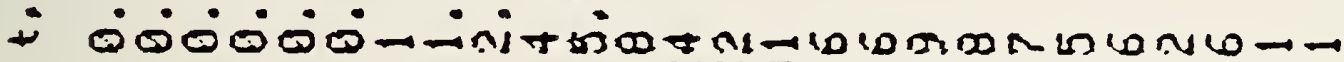

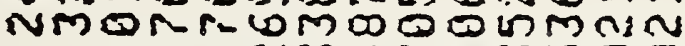

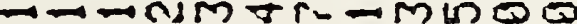

$\rightarrow-\infty$ in

定

$0 \infty$

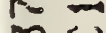

NMNM

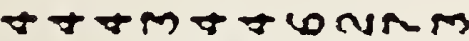

$11,0+\infty 6 \omega-\infty n+a$ and $\rightarrow \infty$

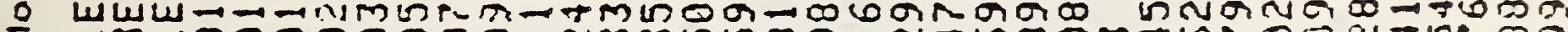

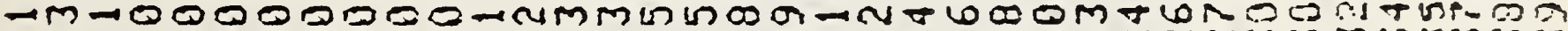

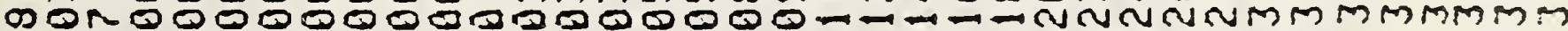

= ம்

L $\rightarrow$ momus

ocongun un an

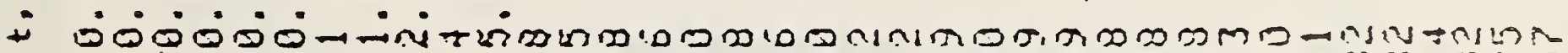

cut IN

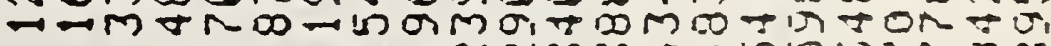




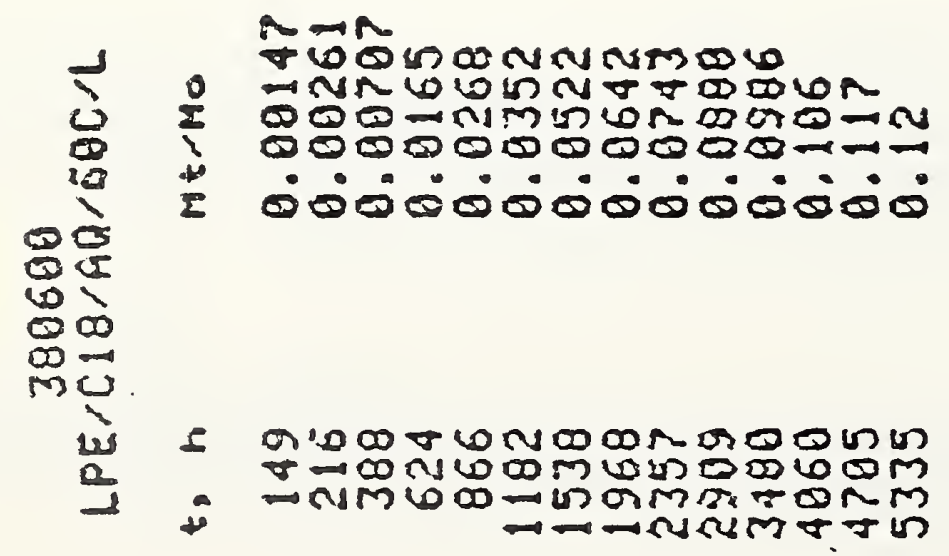




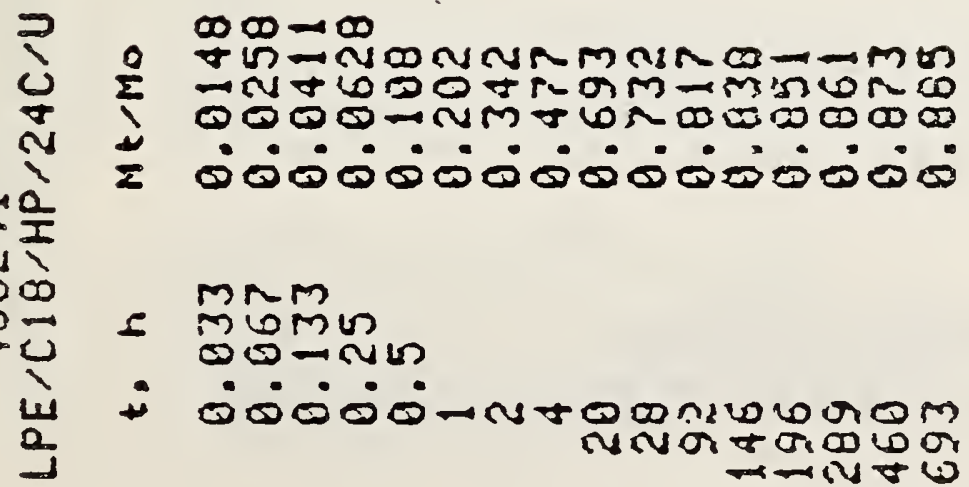



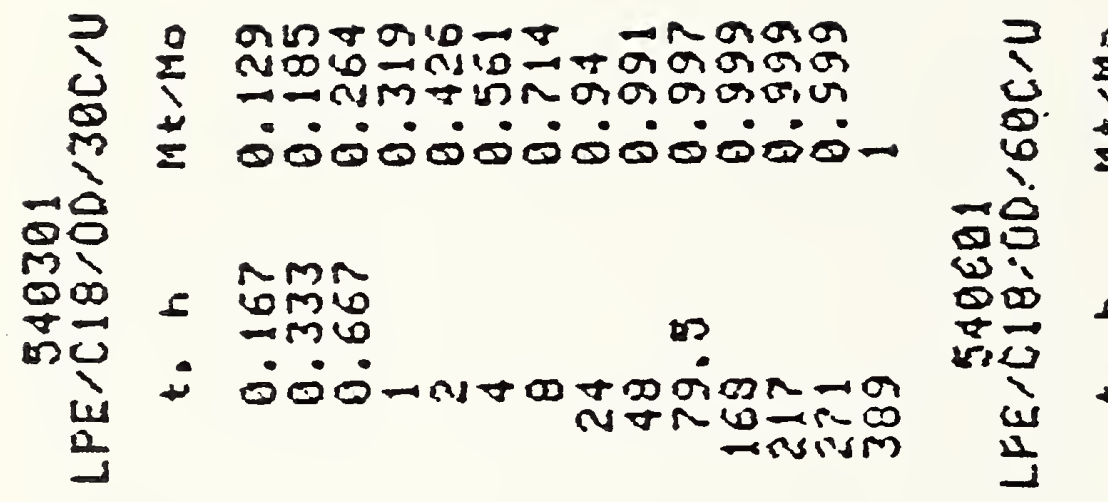

$00.0+M-T+R-7401010.0$

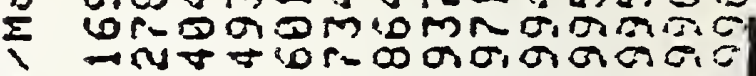

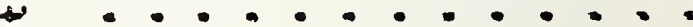
$=00000000000000$

- $\operatorname{lom}$

- Mis un us

- ن்

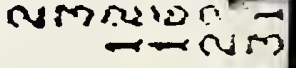

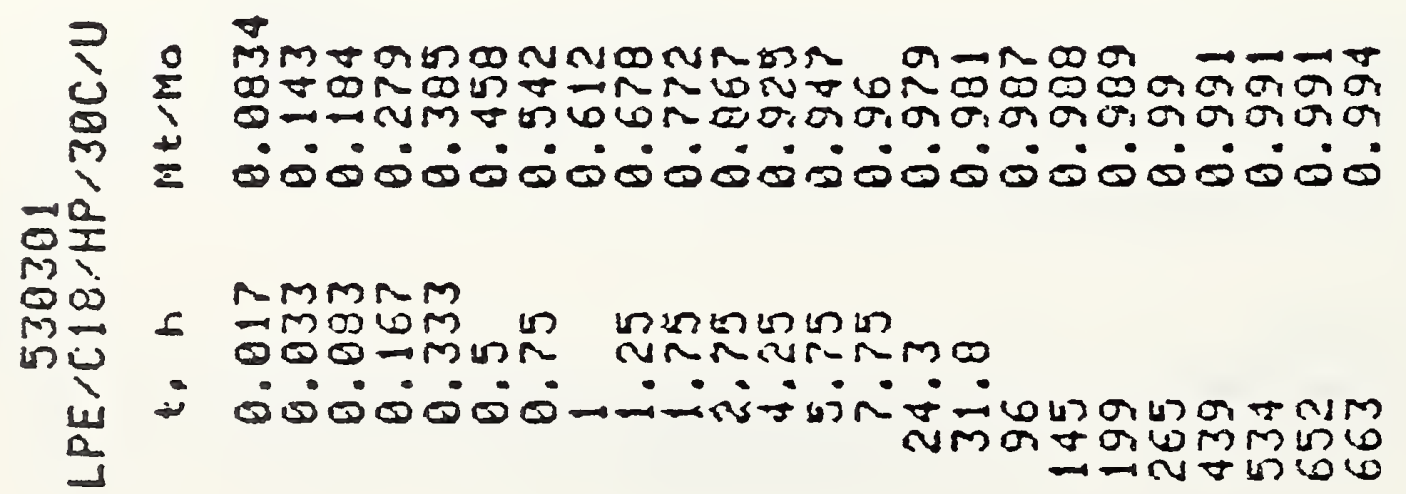




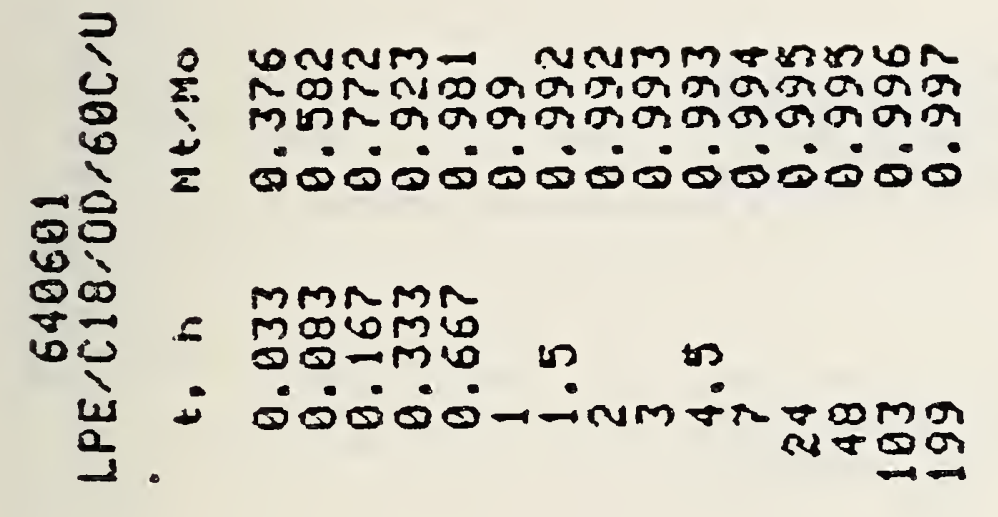

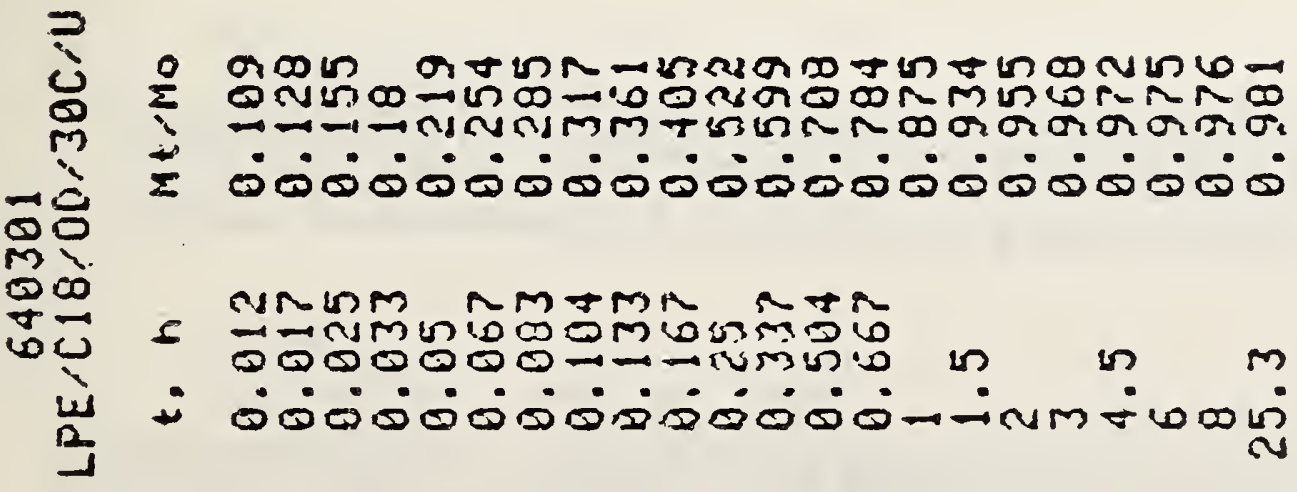




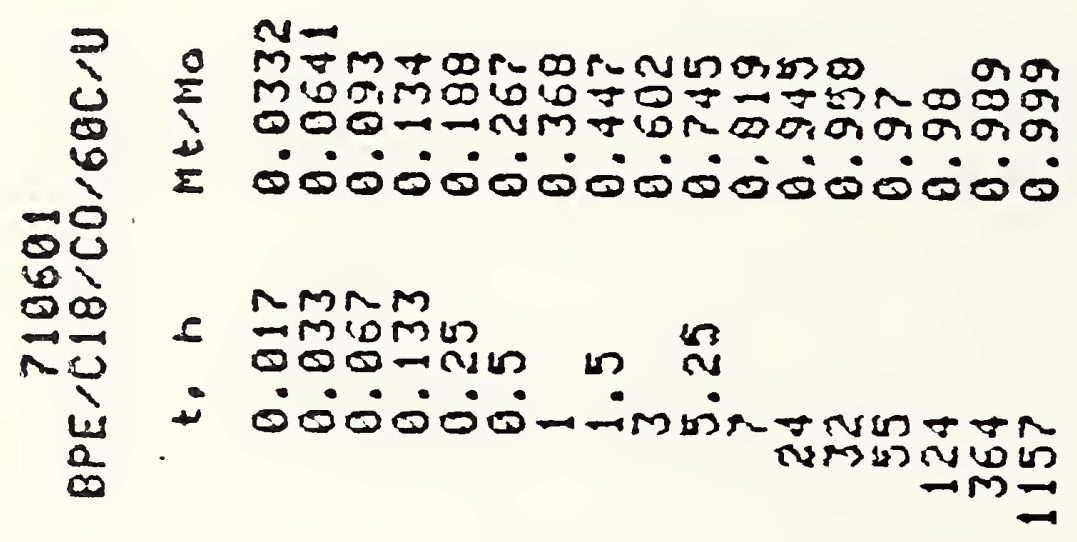

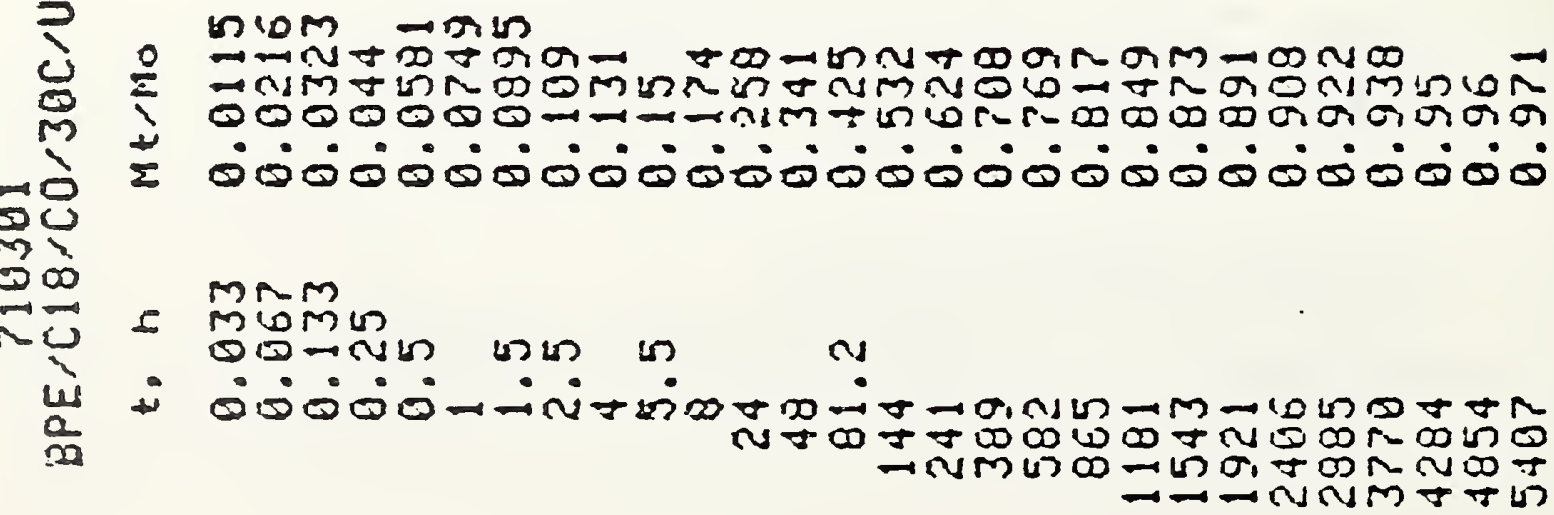




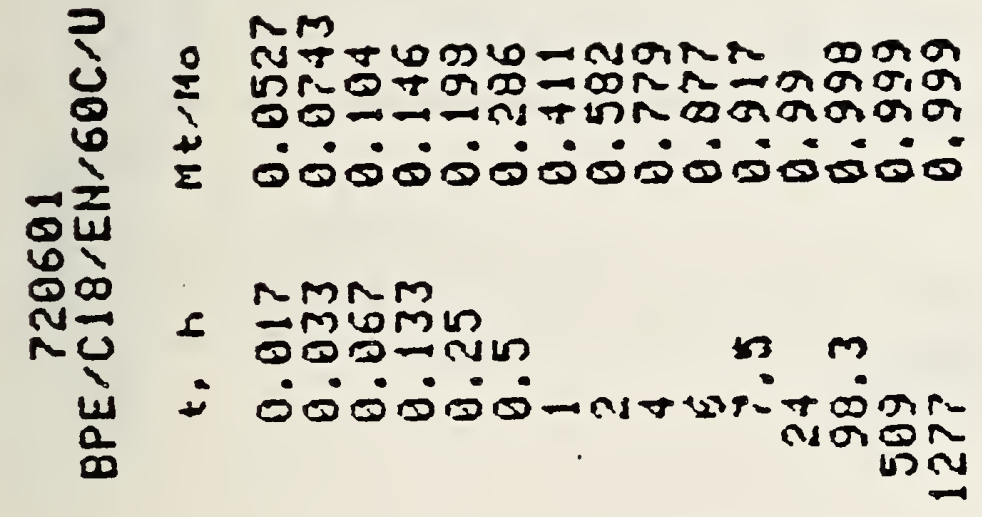

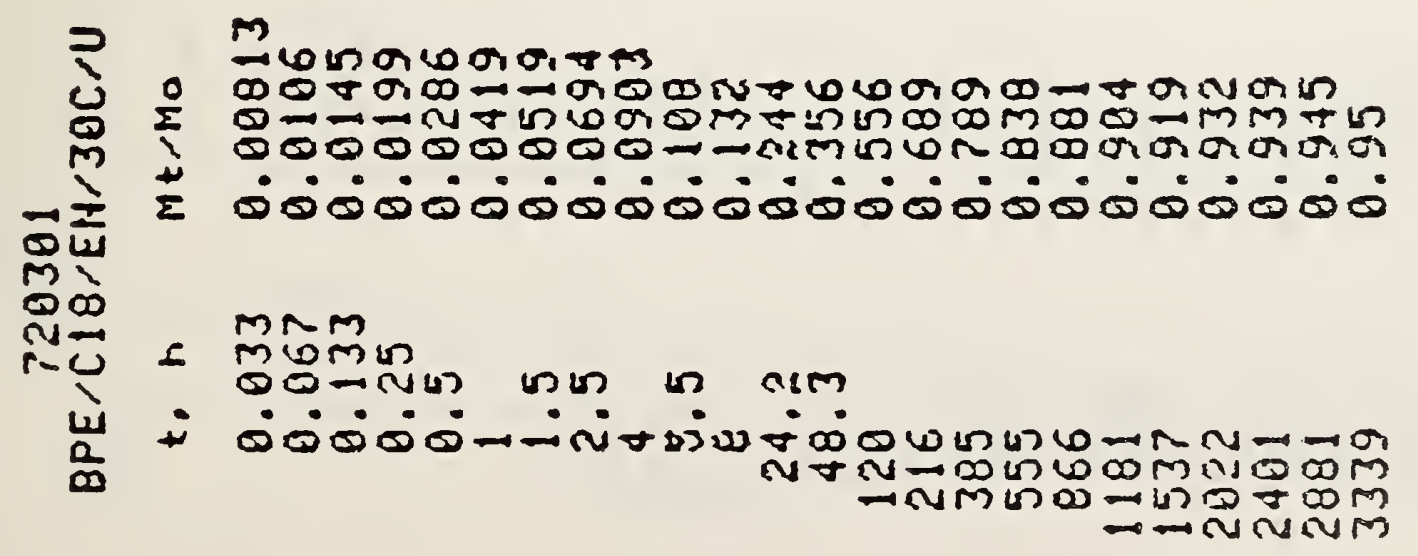



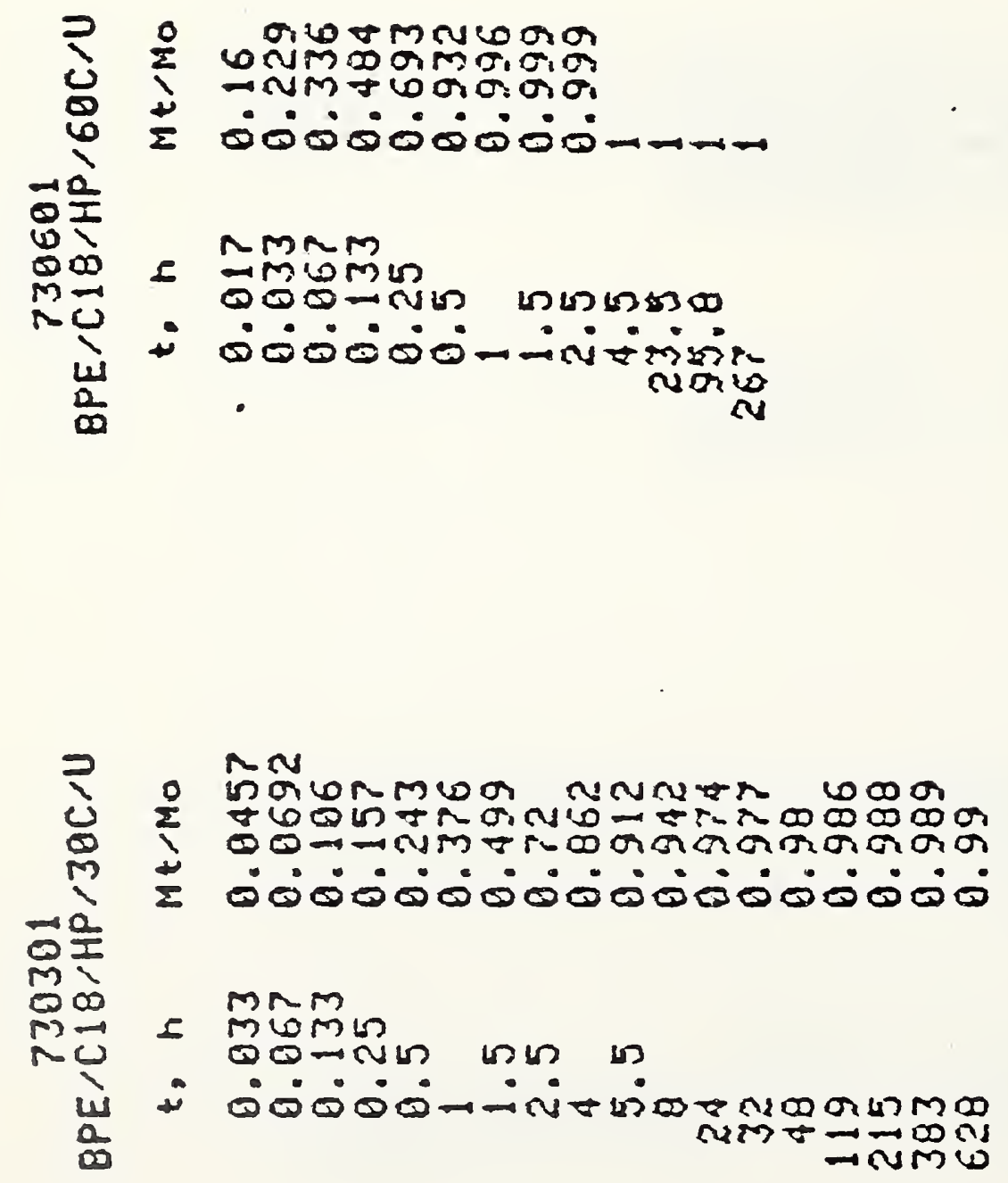


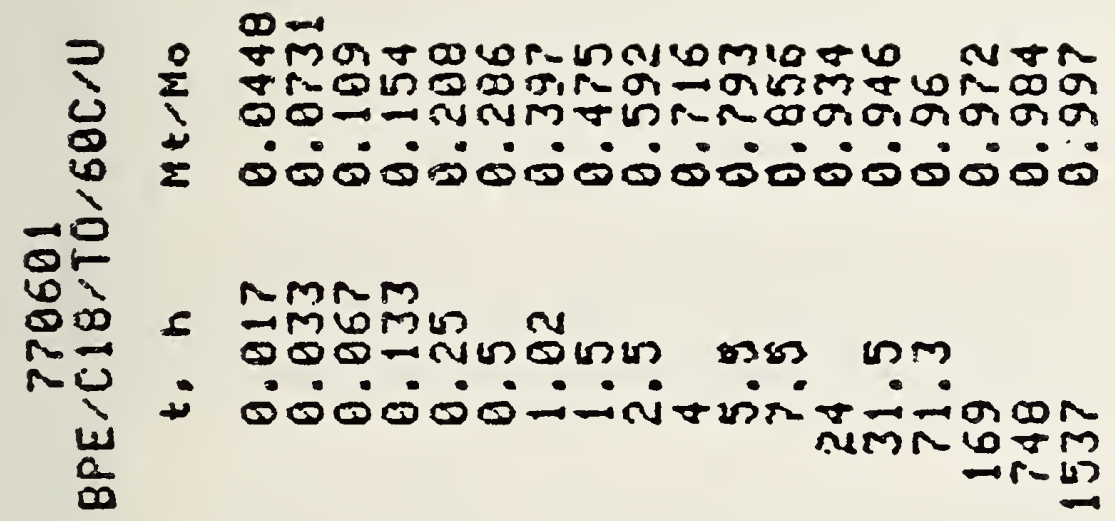

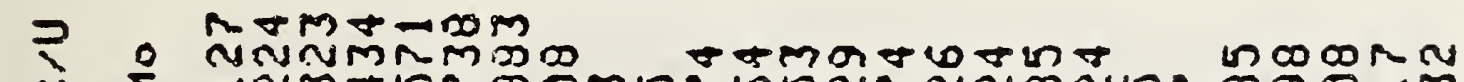

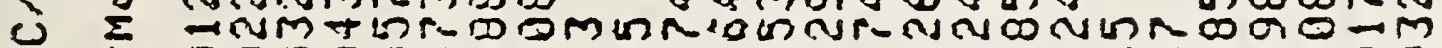

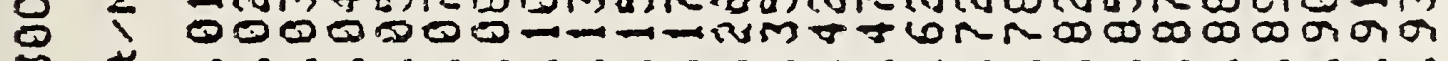

M I

$\infty$

mi

$0 \infty$

Mo $=M M m$

N. 1 MDMU

Nu Domnin un as anut

i) +

"

a

$\rightarrow=\pi N M$
} 


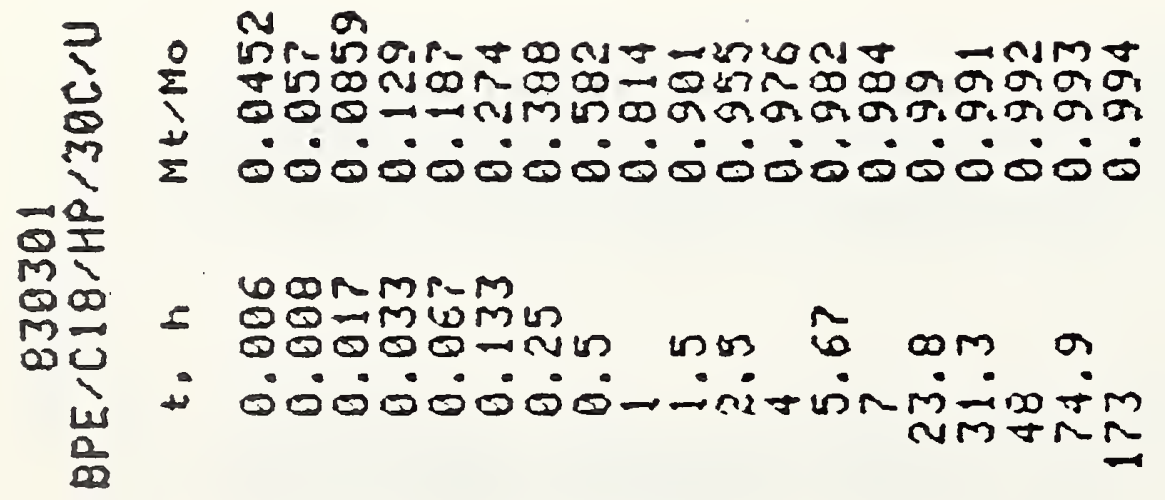




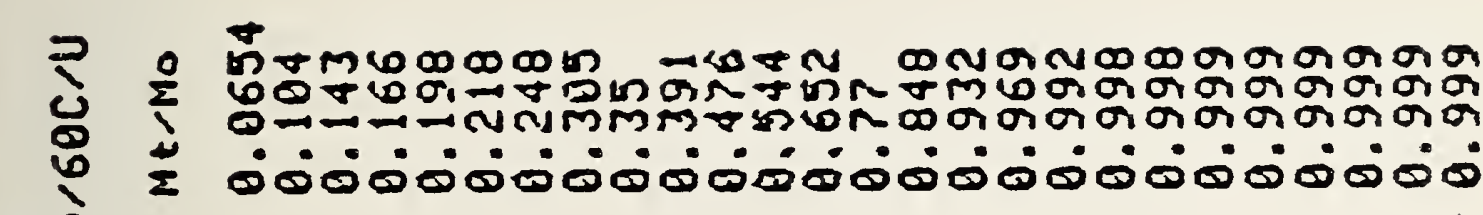

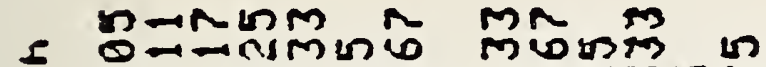

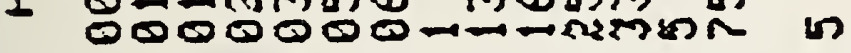

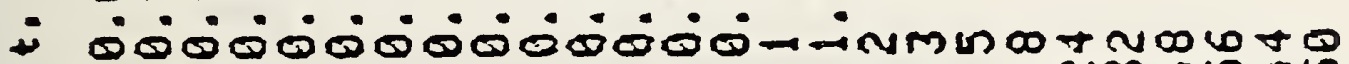

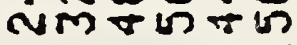

离

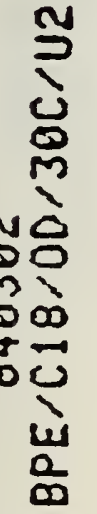

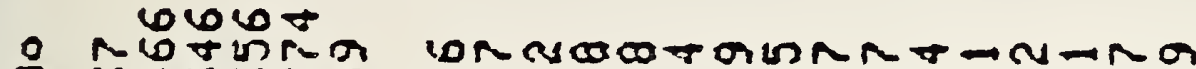

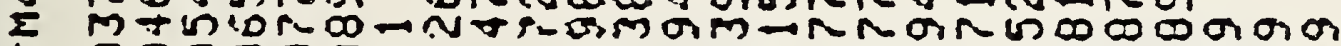

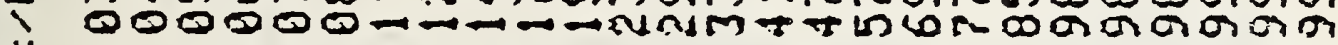
=

- -onkmR mm mmbln

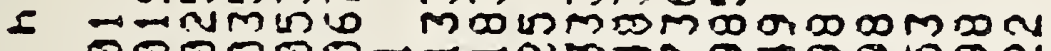
0000D0- - तim, तa

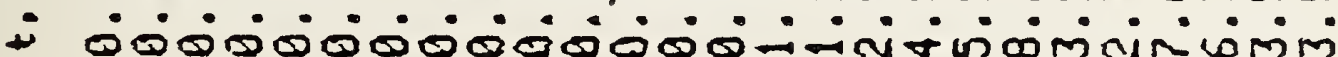

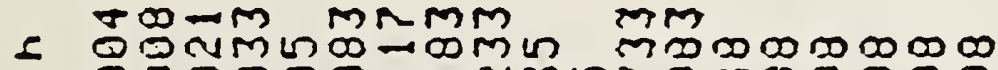

$\ln \pi n$

- NDomon wr Fis

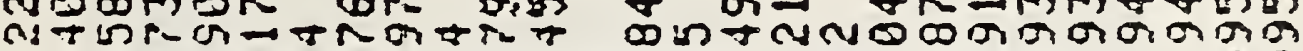

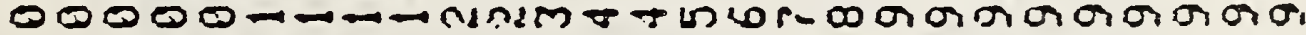

乏 


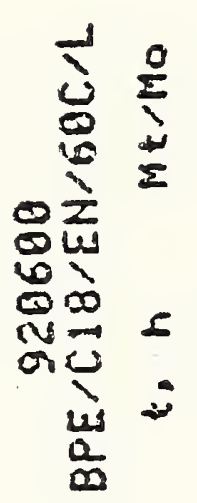

$\rightarrow$ in

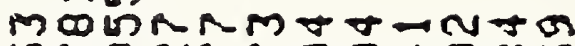
URondr $000-\pi$ -

$\ln 6 \pi \rightarrow \pi \sigma$ ararararara

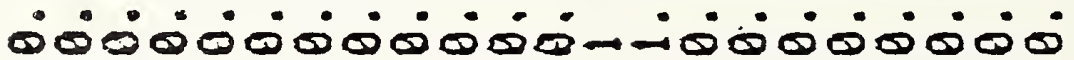

$N M \cap M$

- Mvomis

$000=04$ ne

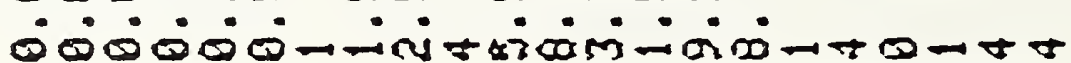

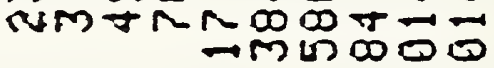

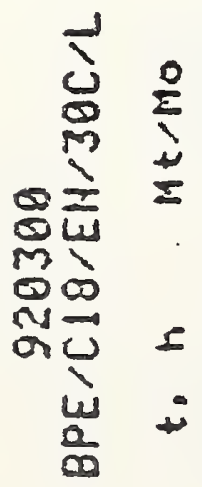

wownom

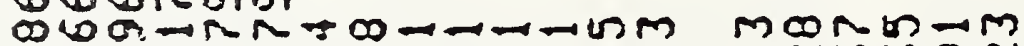

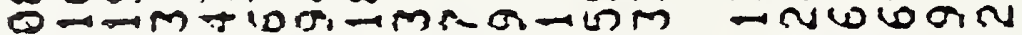

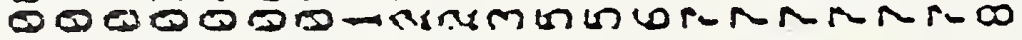
- 000000000000000000

$N m$

Wmine à ñ $000 \mathrm{~m}$

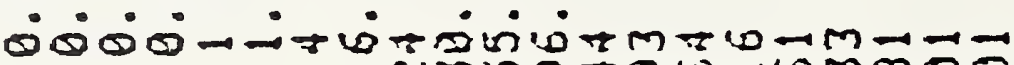

लmuक 


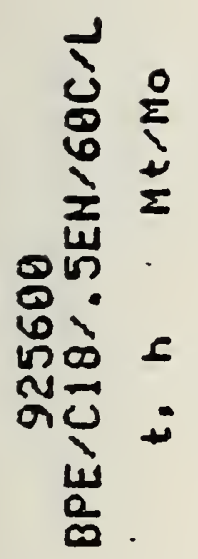

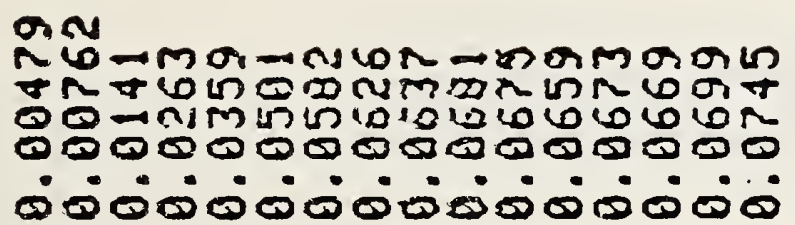

$m$

mis

ines un us ando

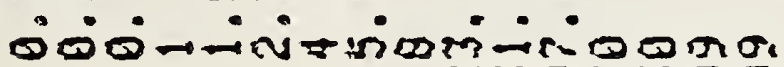

rmangis

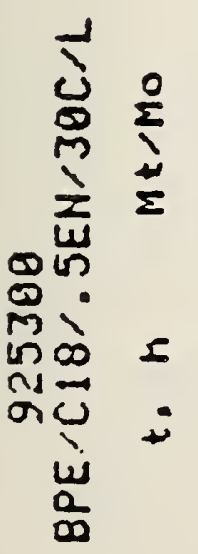

on

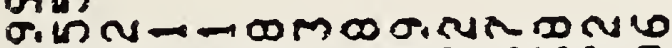

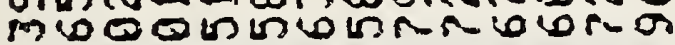
$00-1-1-1-1-1$ ద ن்

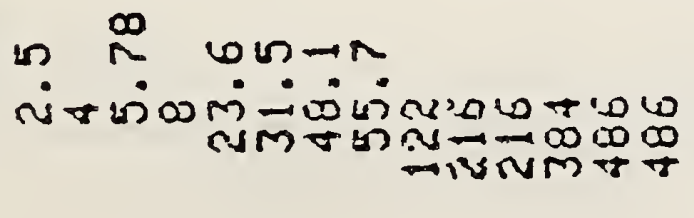



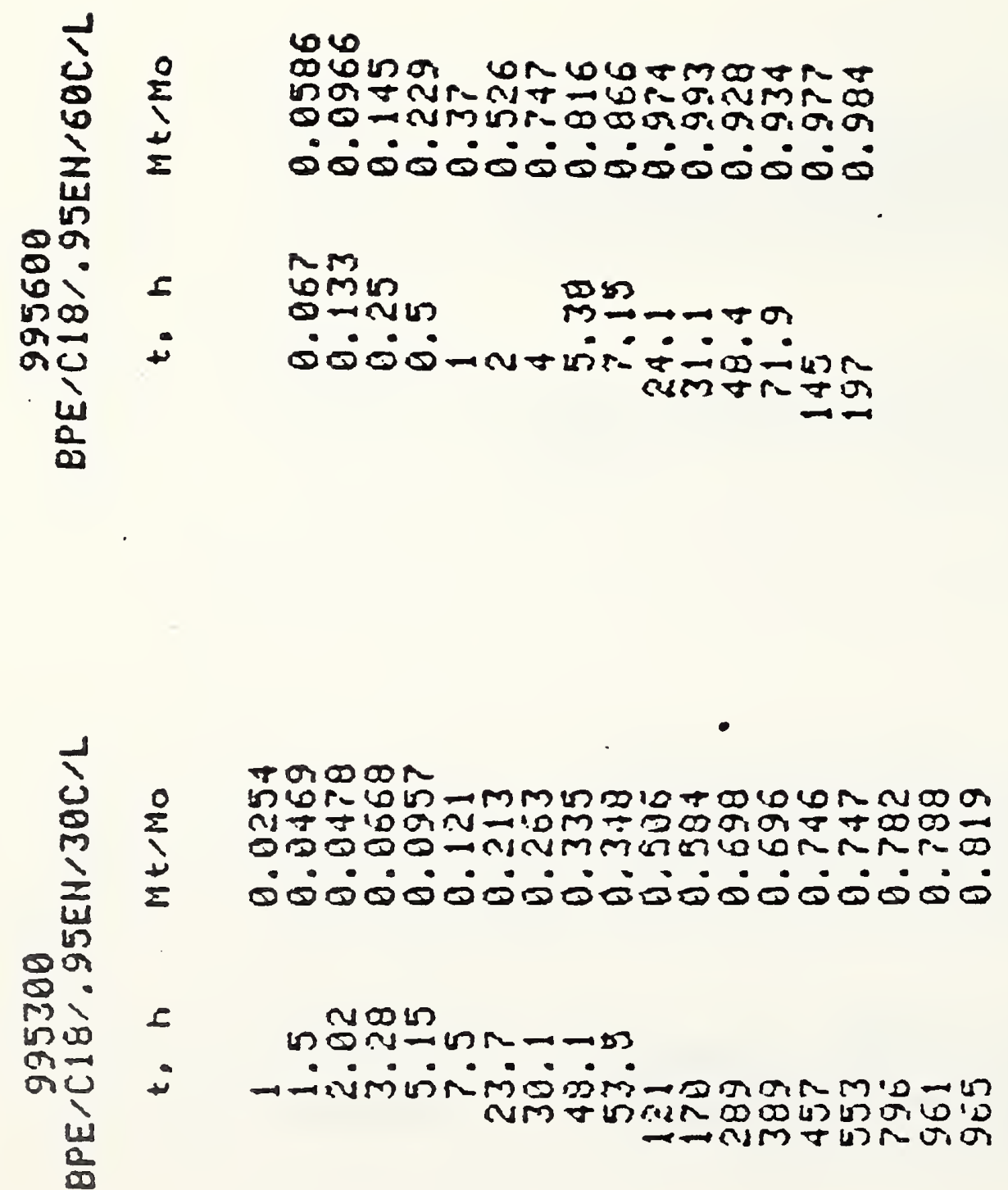


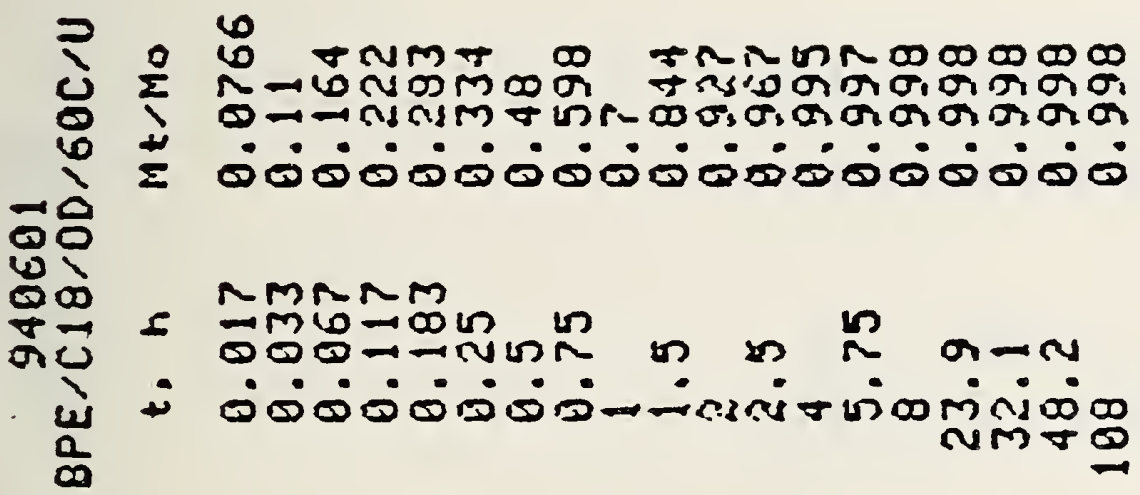

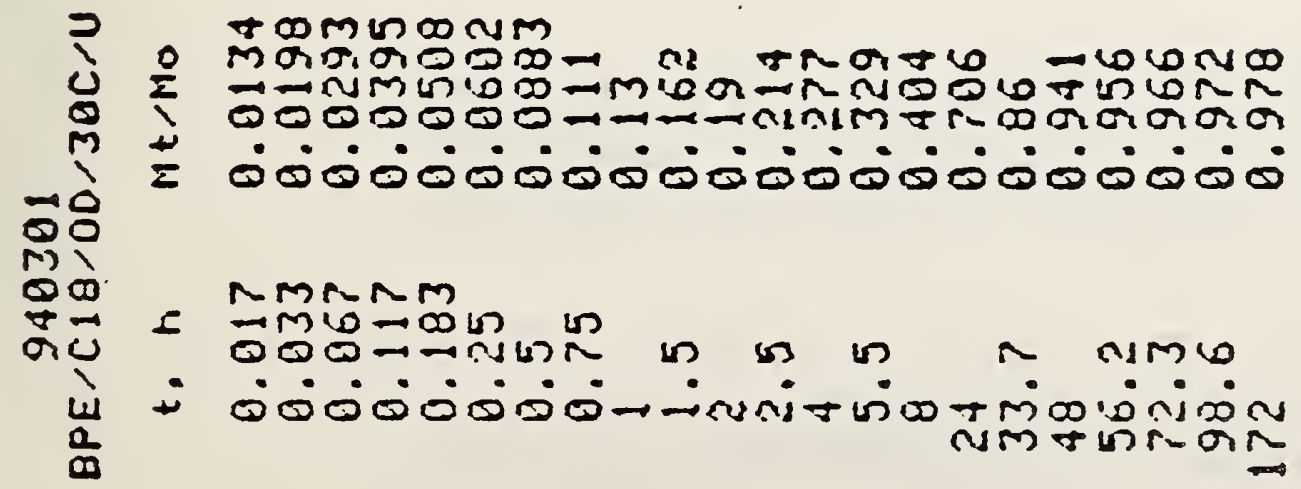




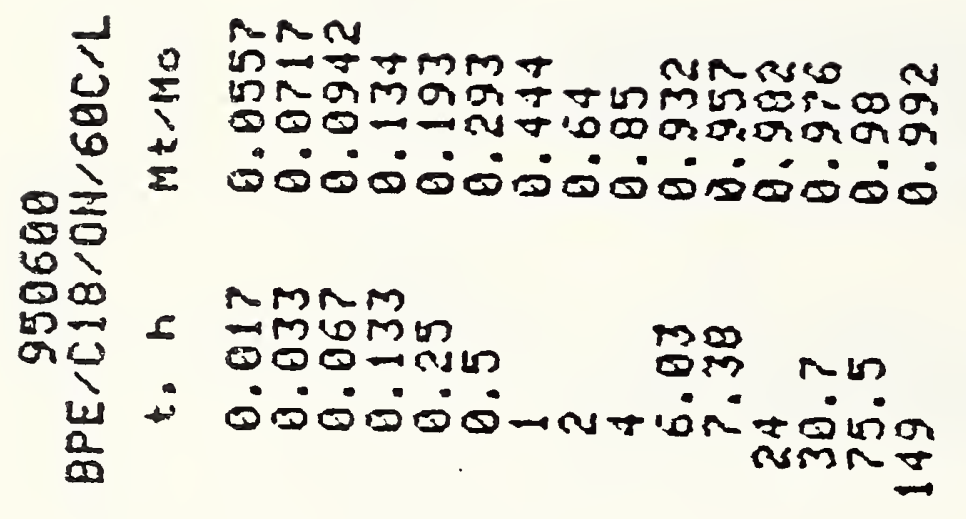

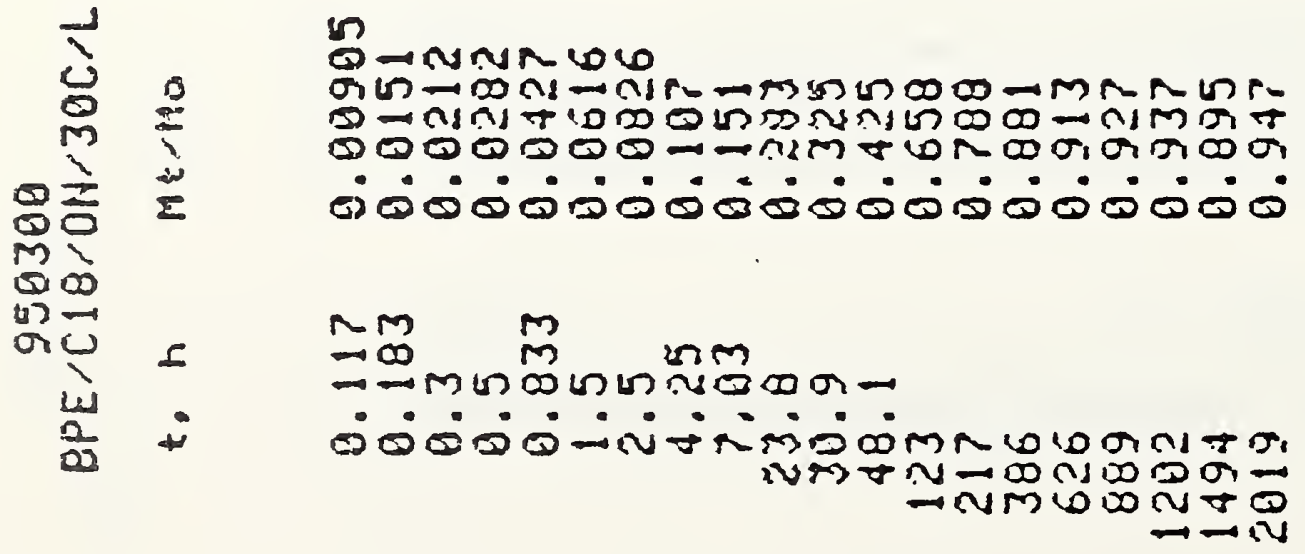




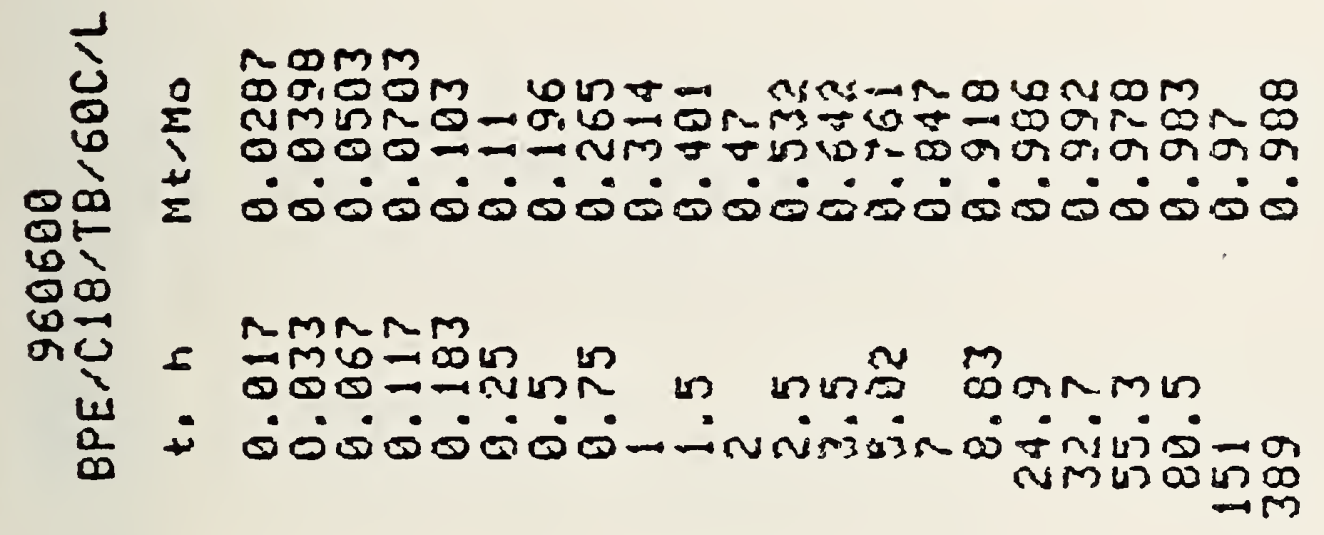

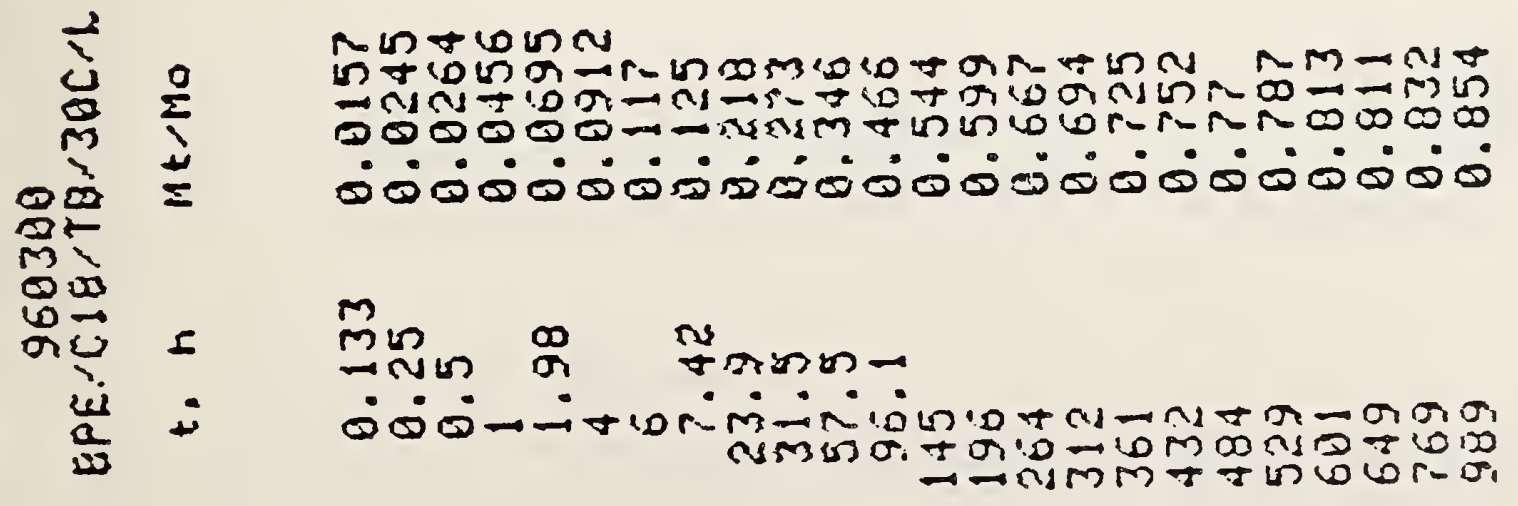




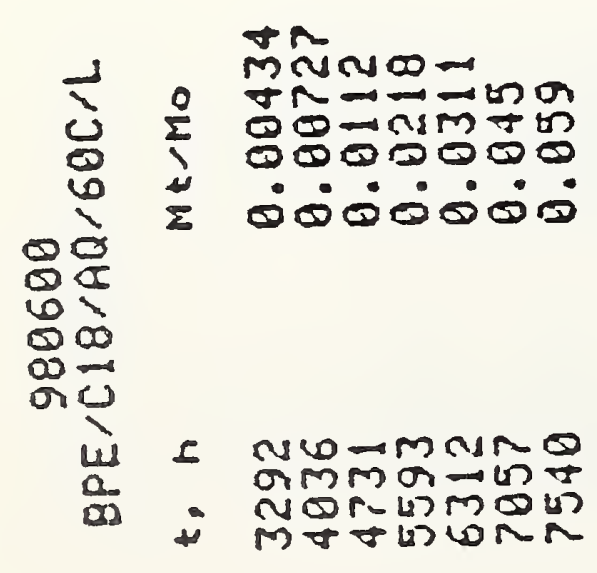



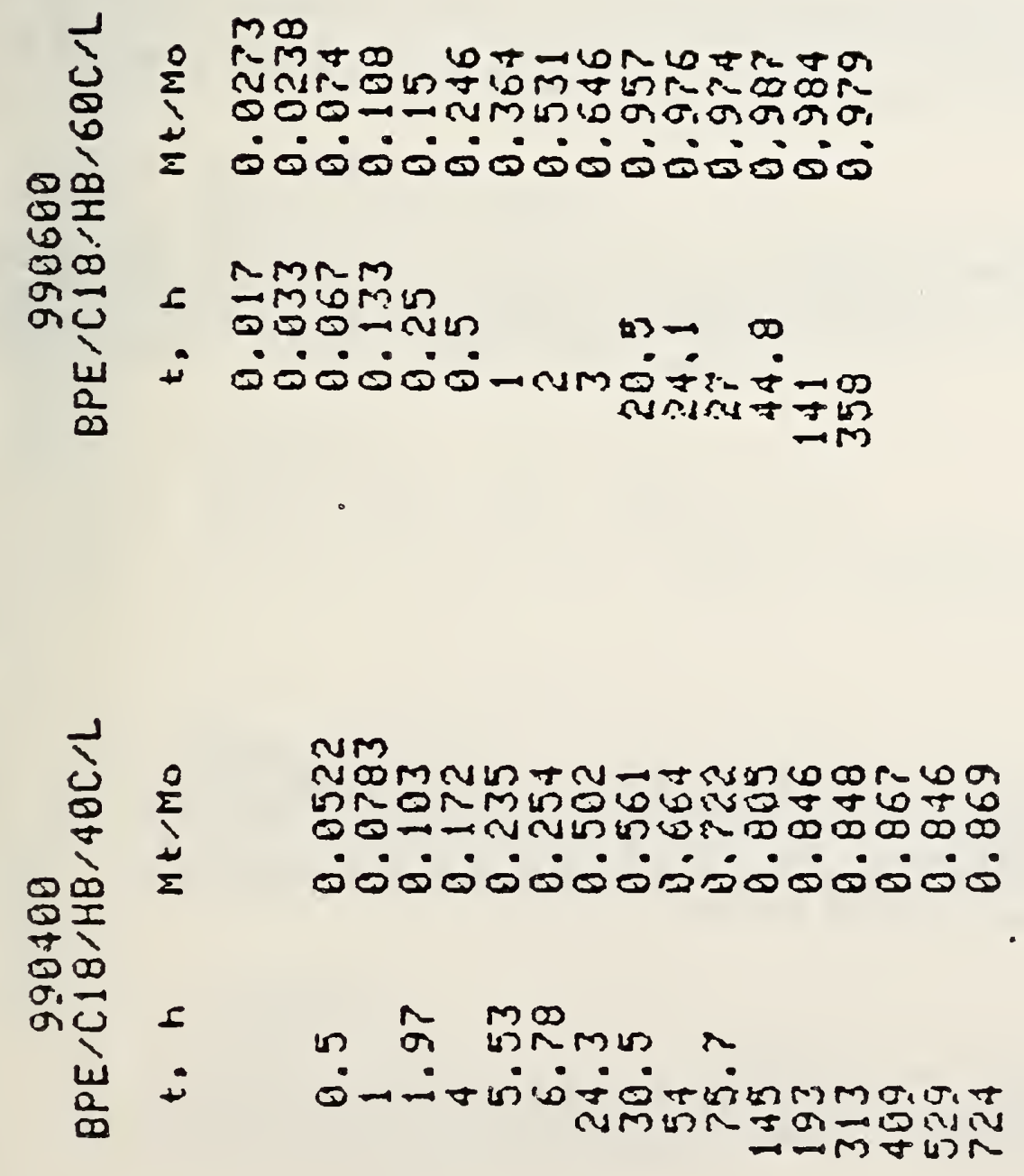


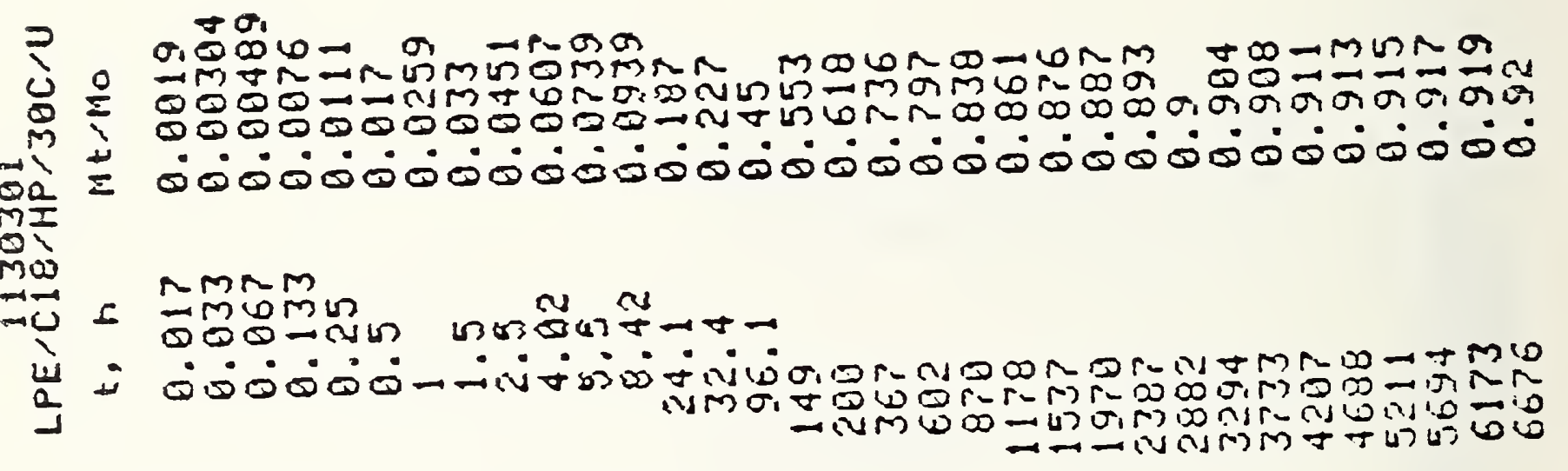

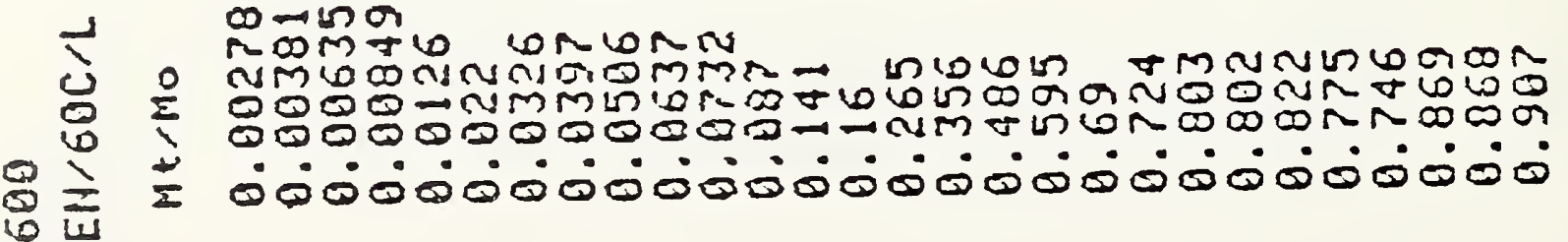

0

ก:

$\Rightarrow \quad$ RMNM

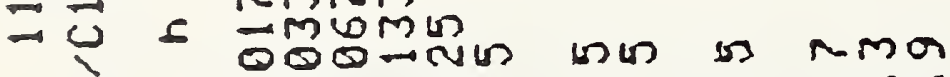

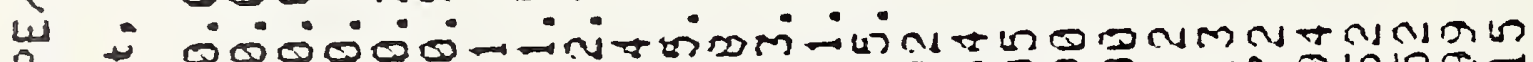

I 


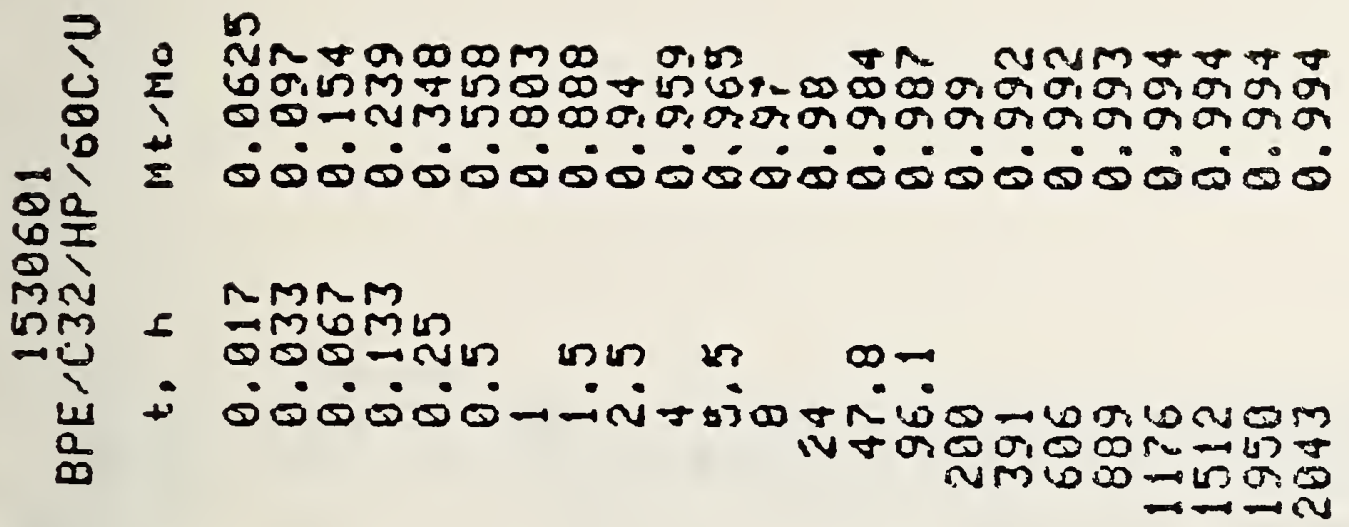

$+\sin \omega$

i) $\min =-1-\infty \pi \infty n$

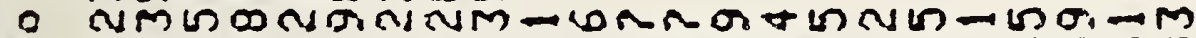

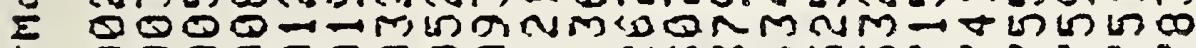

12

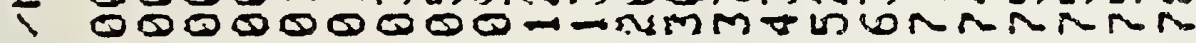

$\infty 0$

= ن

$0 \div$

mas

m

-i. I. IMn

I Imbmen os non

000 -

u -

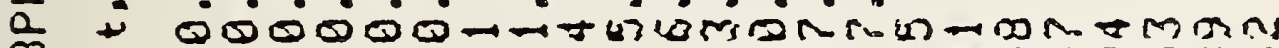

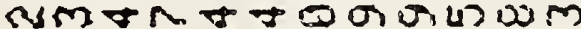

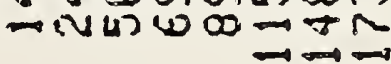

$$
\text { rmam }
$$

I

- 0007rín inu un $M$

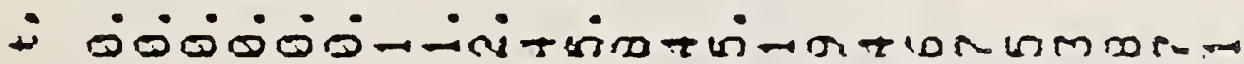

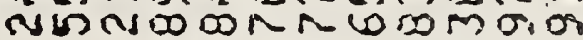
$\rightarrow$ N 


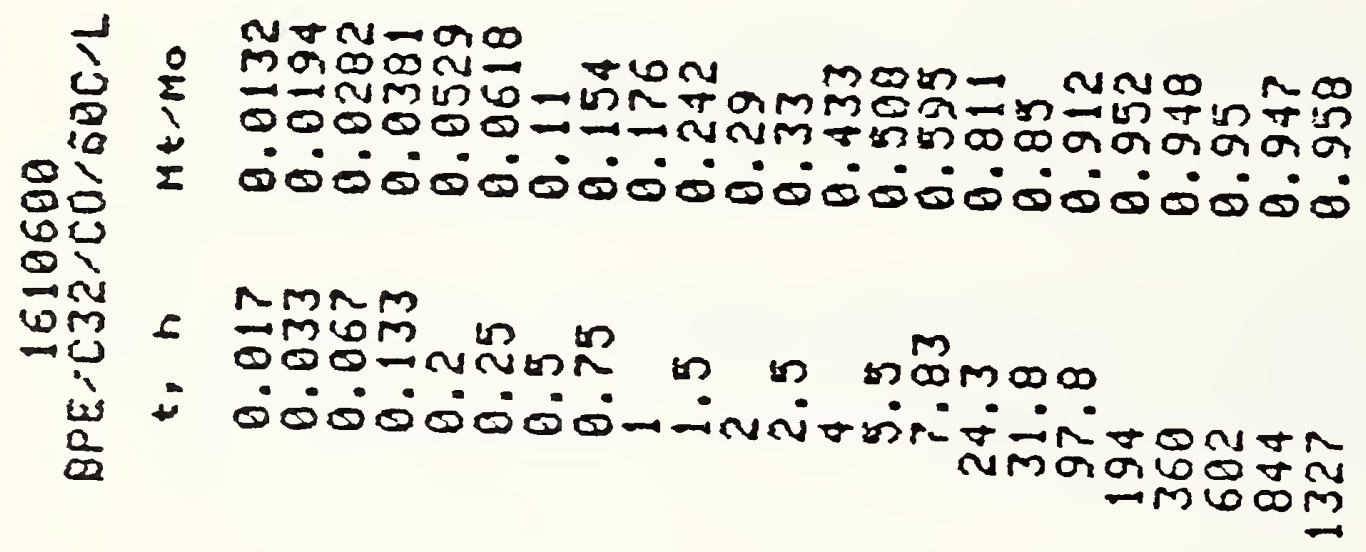

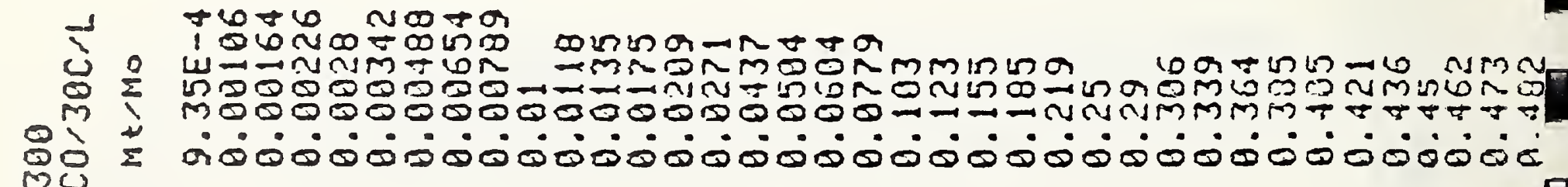

01

$=\pi$

wim

$\rightarrow 0 \sim M n M$

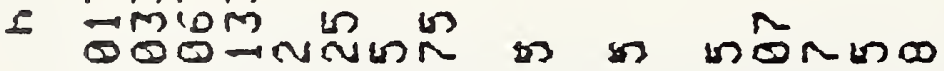

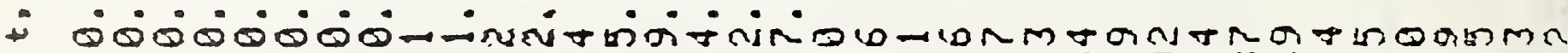

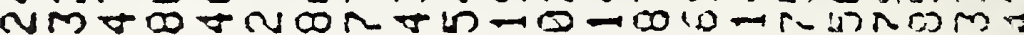
- NMU

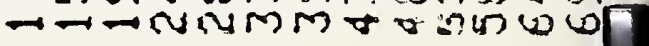




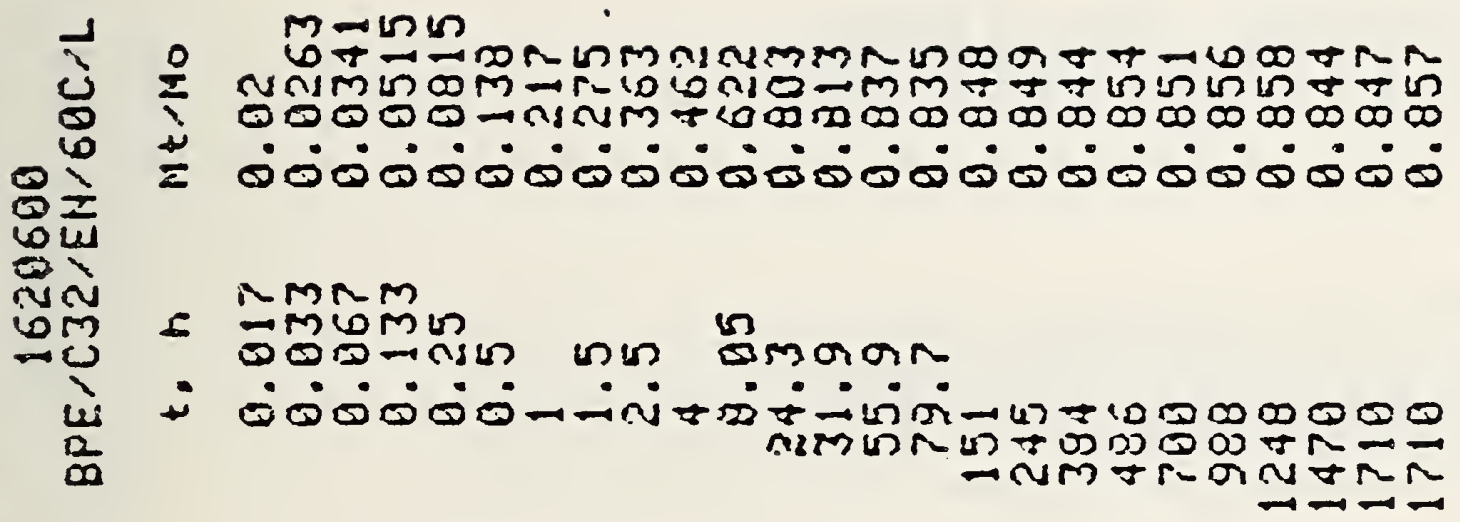

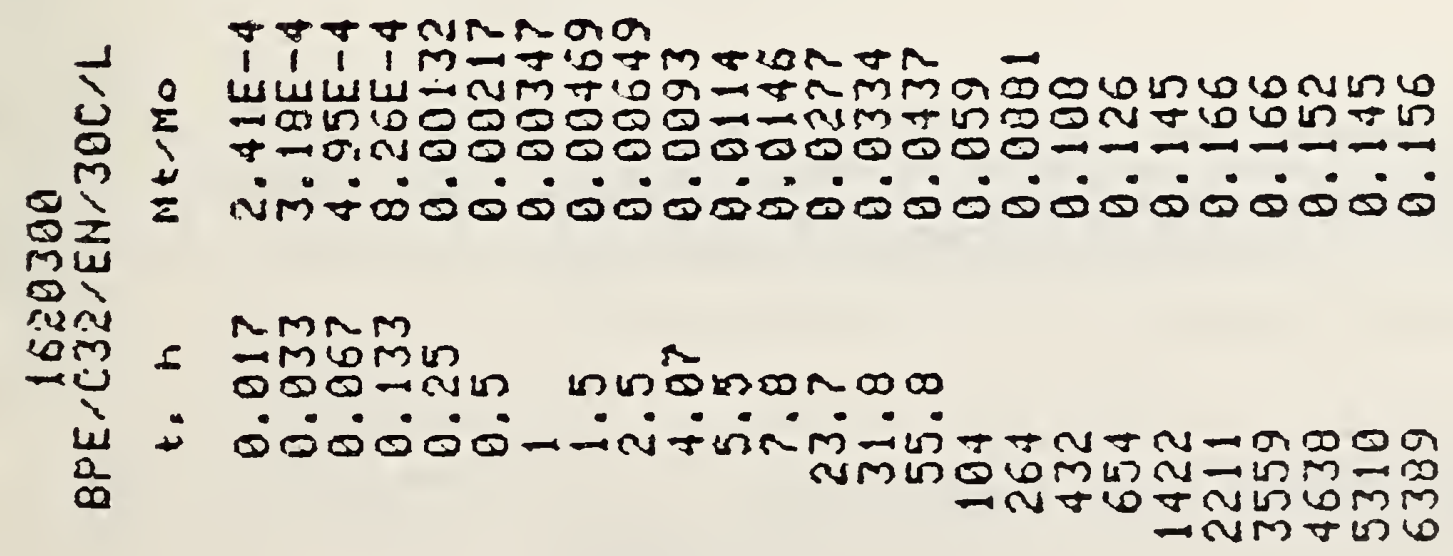




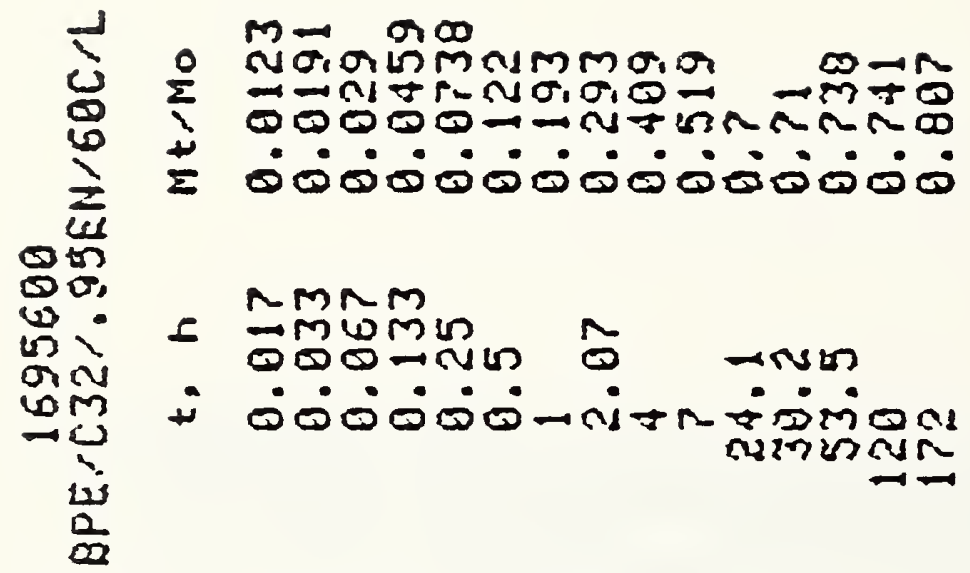

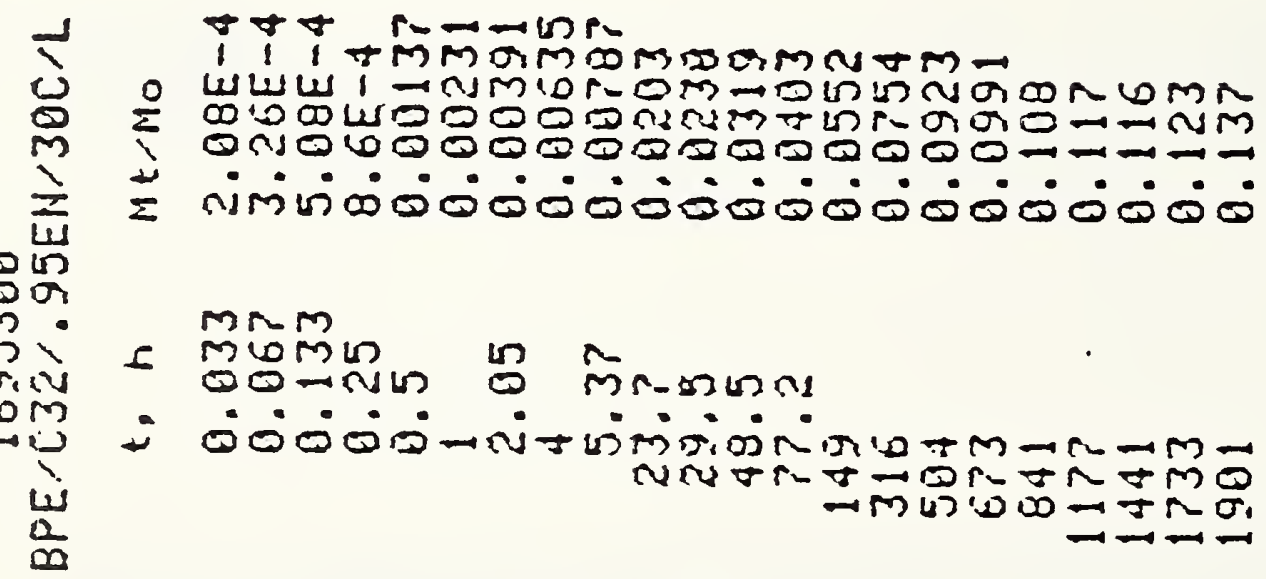

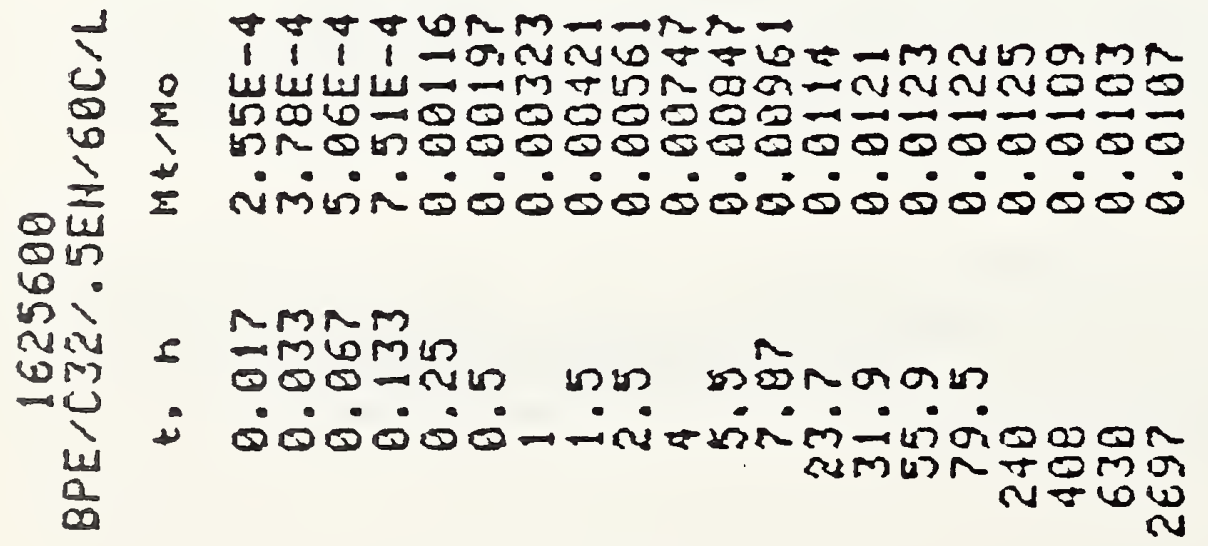




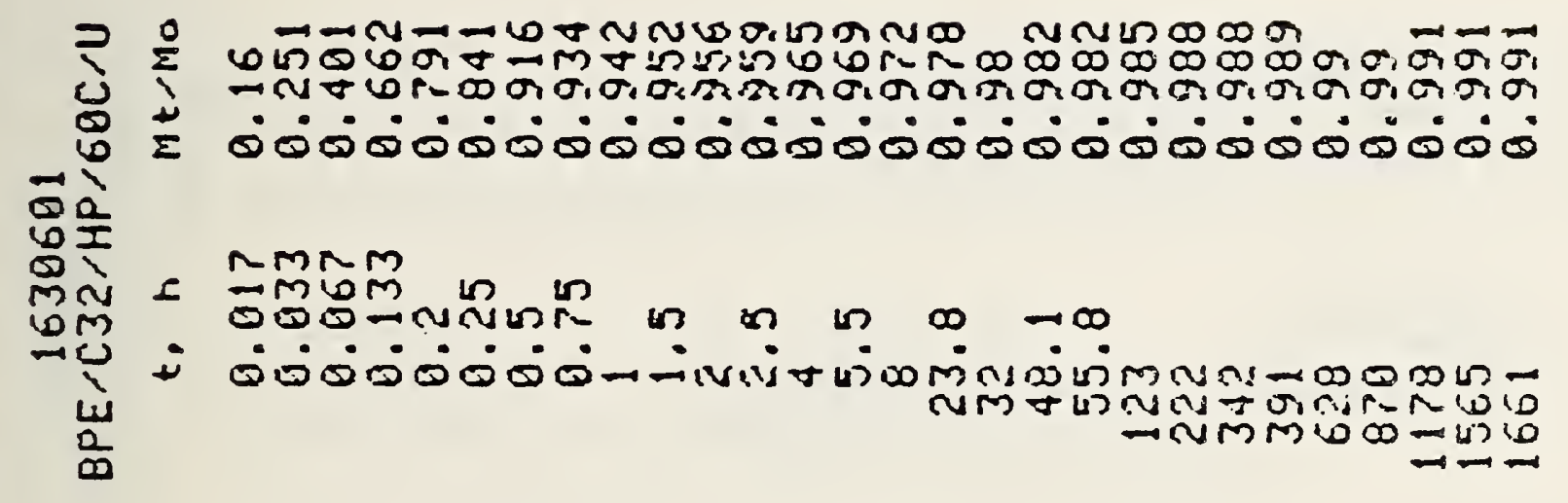

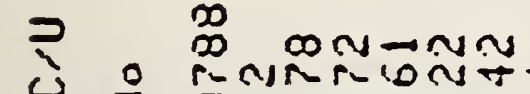

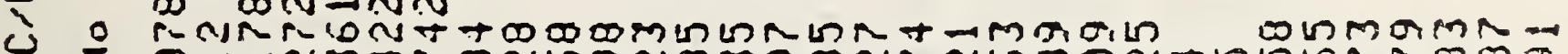

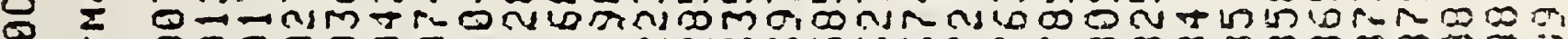

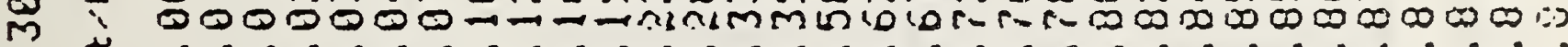

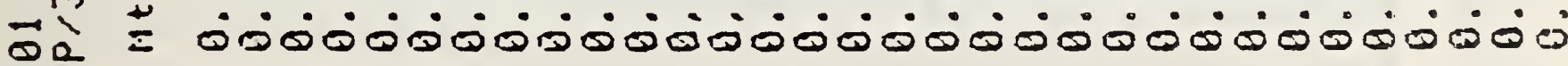

$m$ 垔

$0 \frac{7}{2}$

$m(1$

om $M M n$

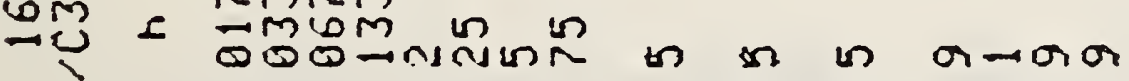

u

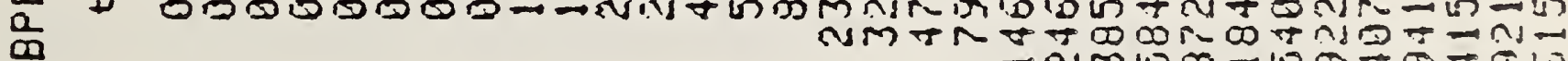




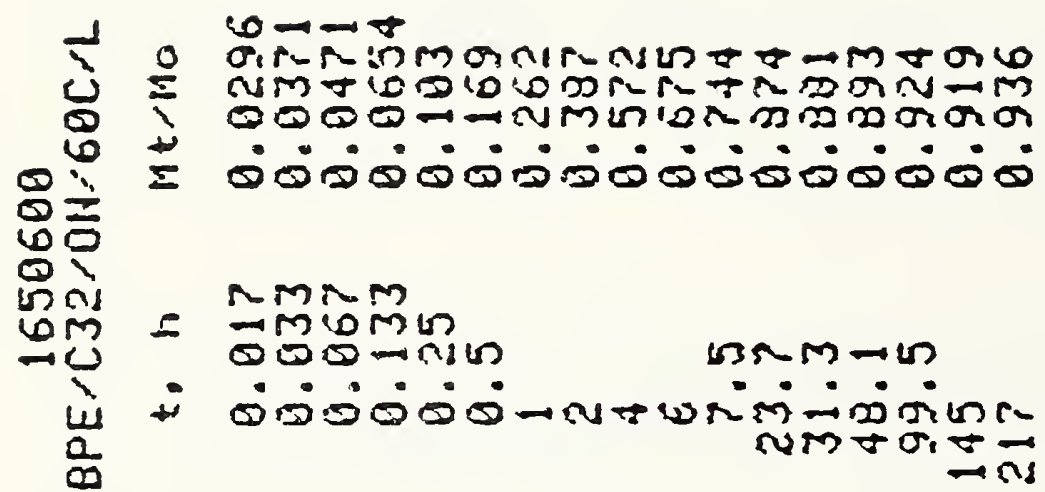

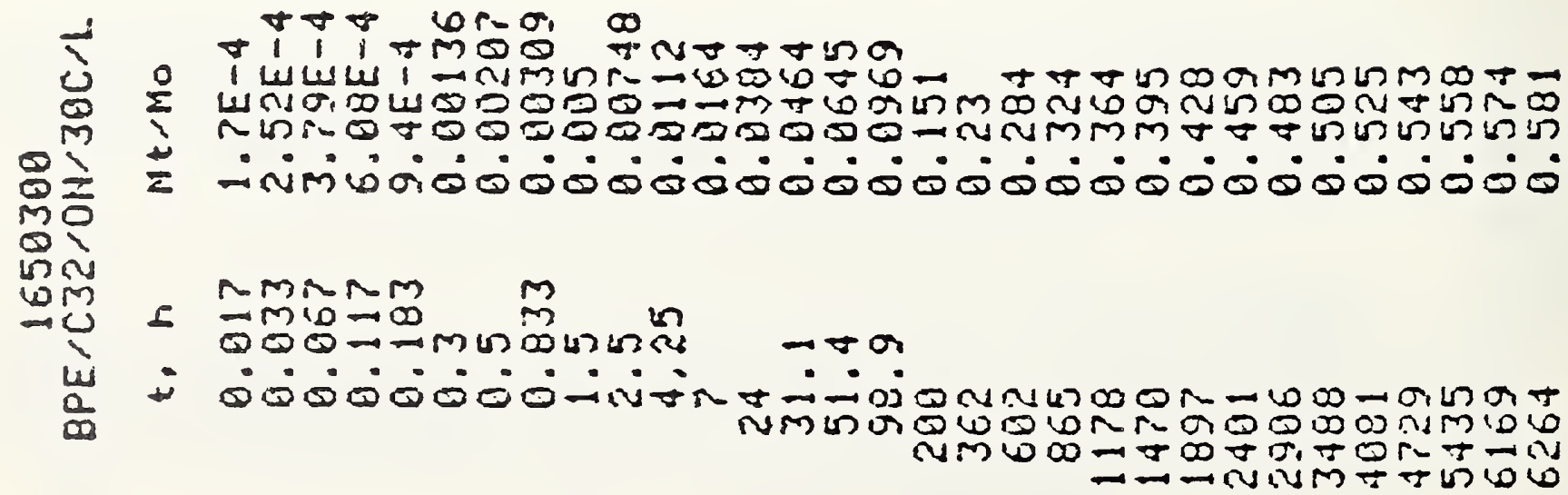




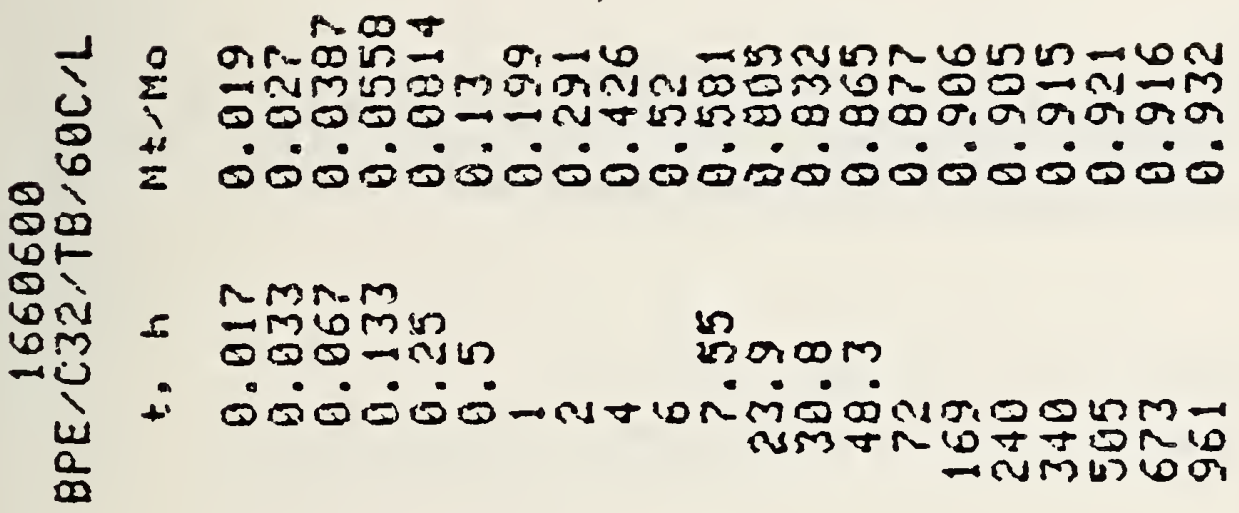

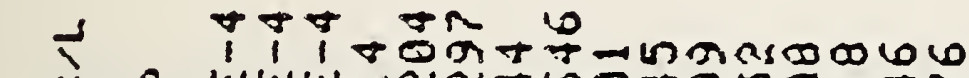

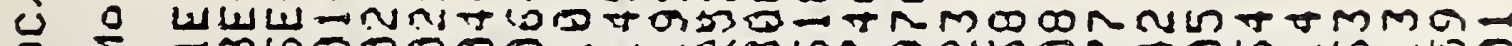

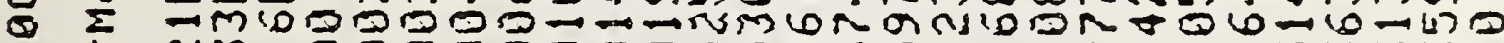

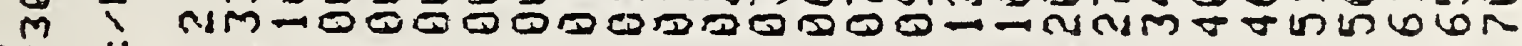

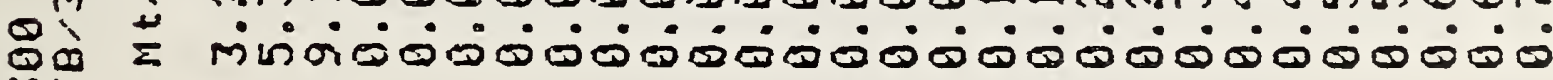

Mt

o)

wis

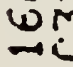

-

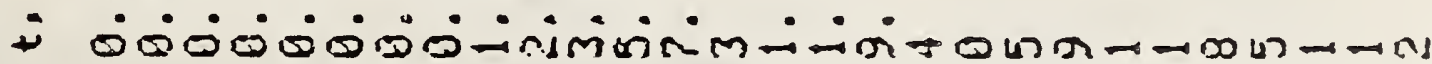

NMUR RIDRNOMNDM-

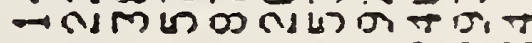



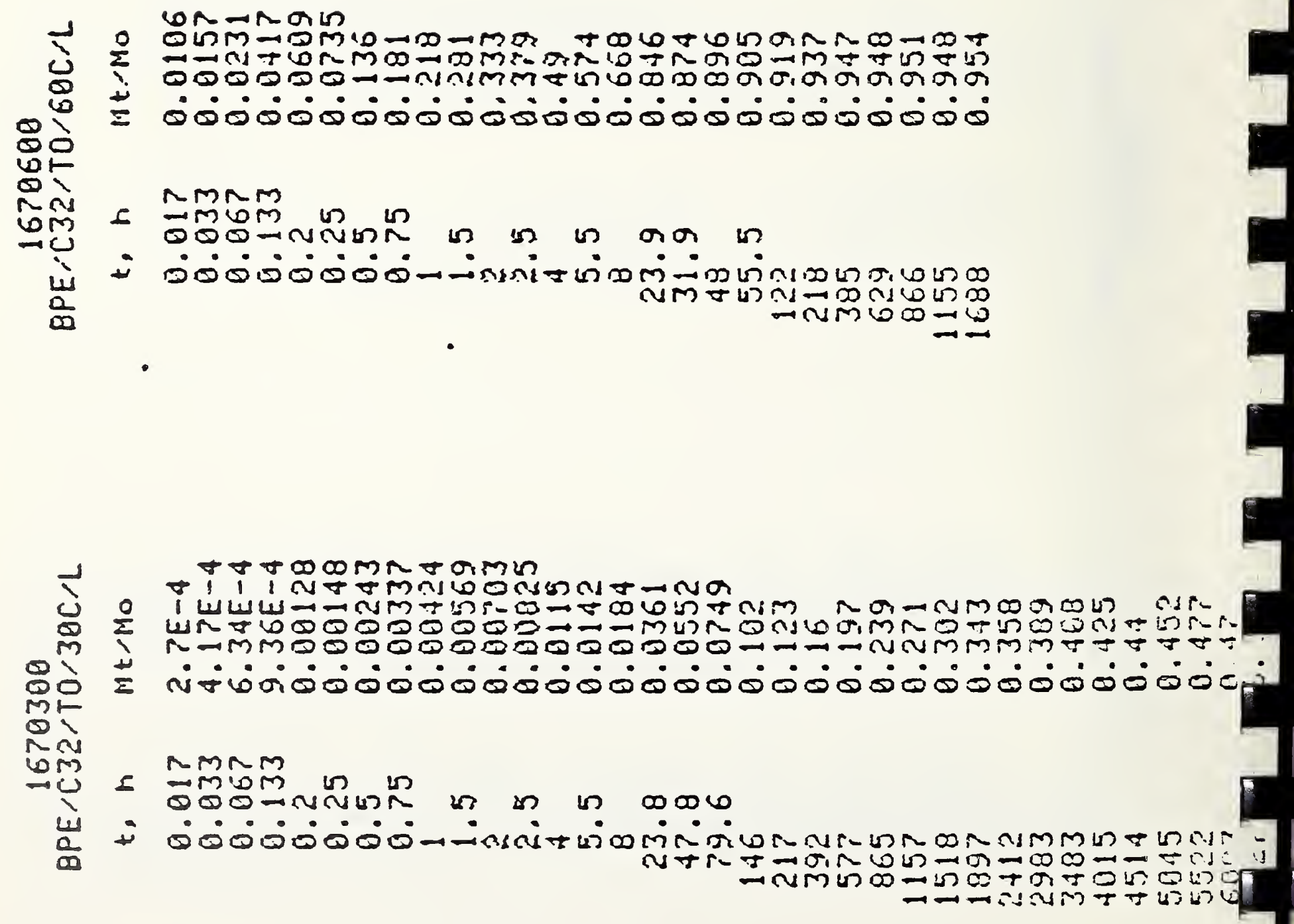


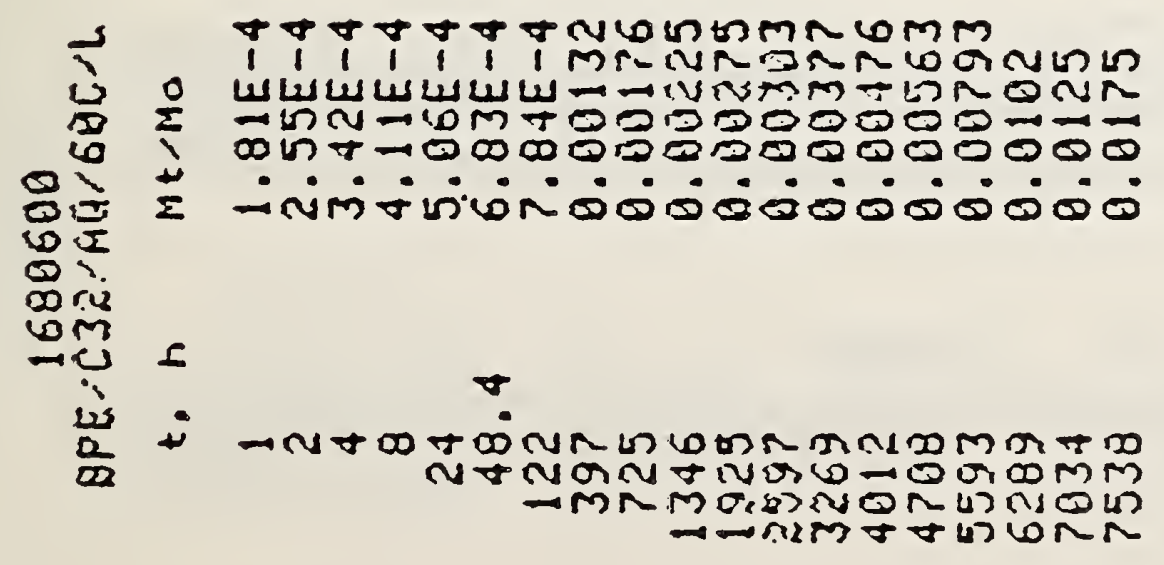




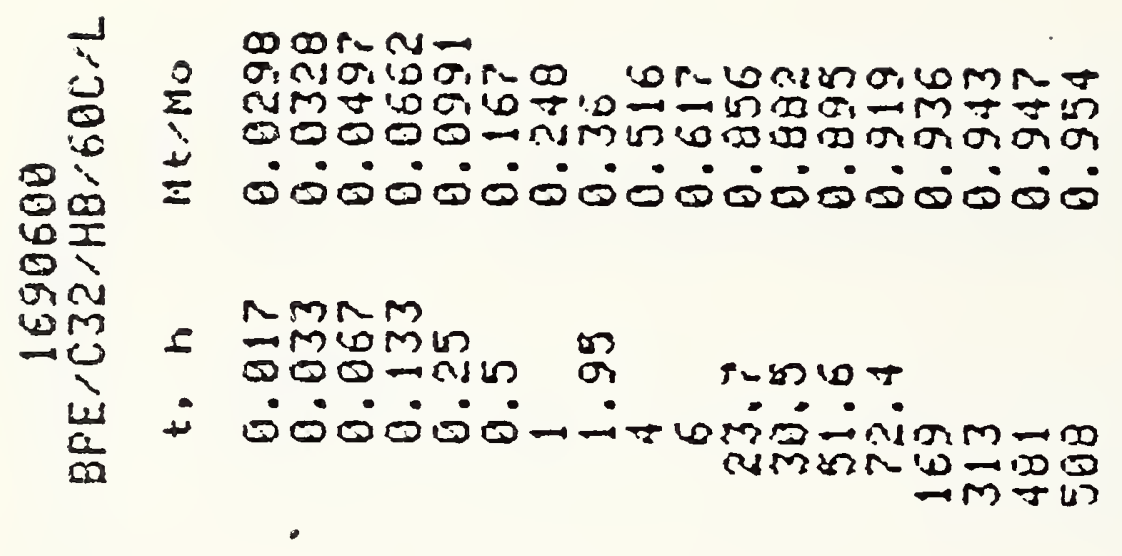

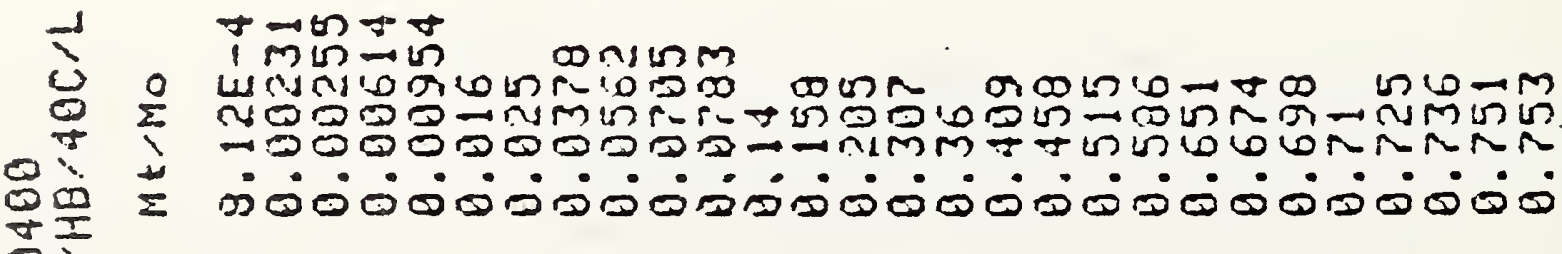
0

oxim

WOM NMN

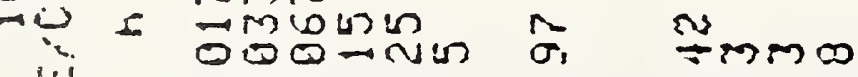

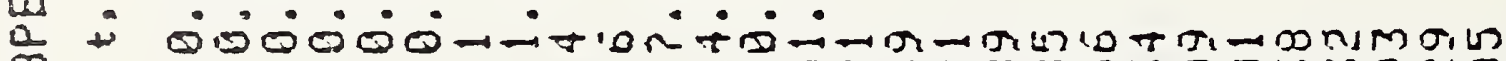

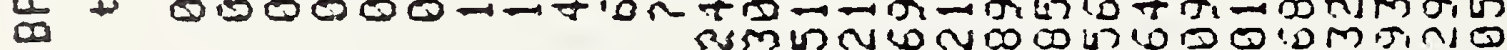

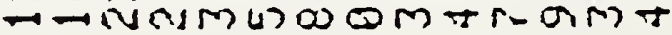




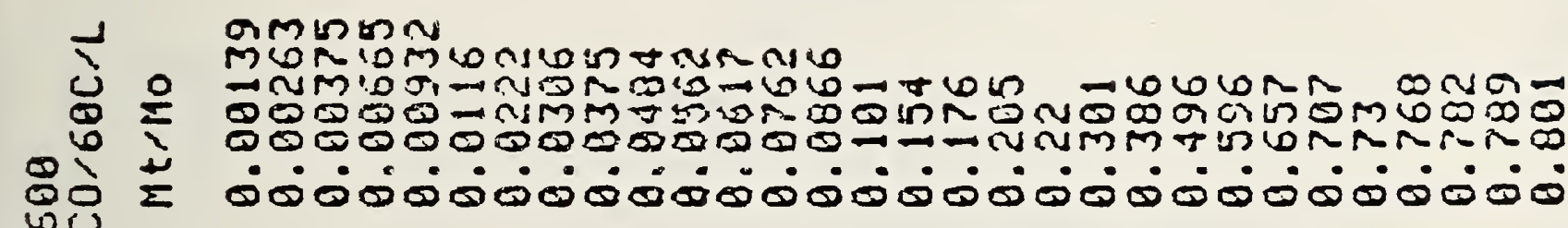

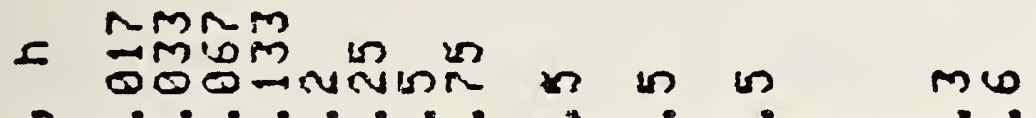

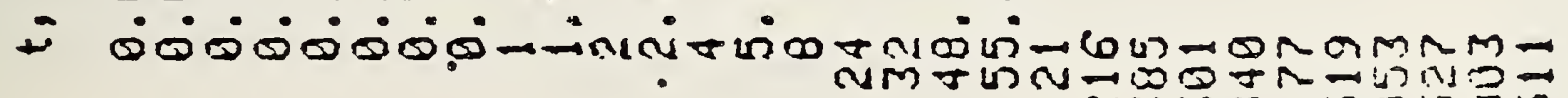

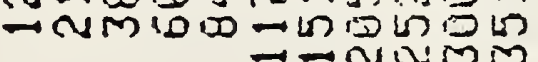

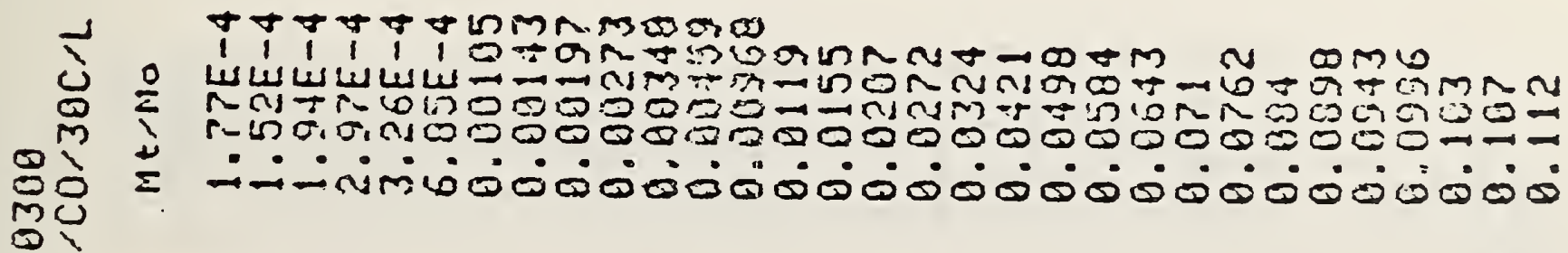

$\rightarrow$ nis

Vim $N M n m$

-is 5 oniman

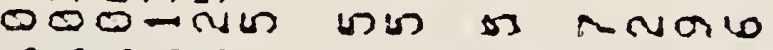

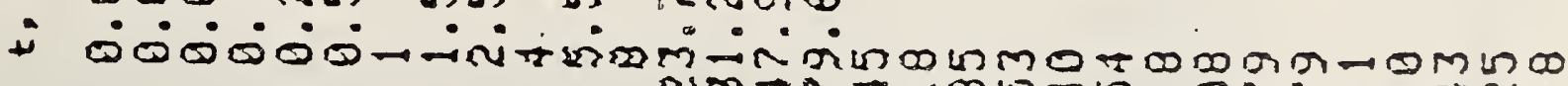

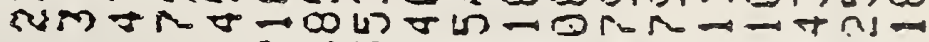

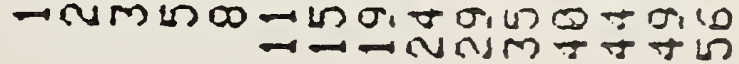




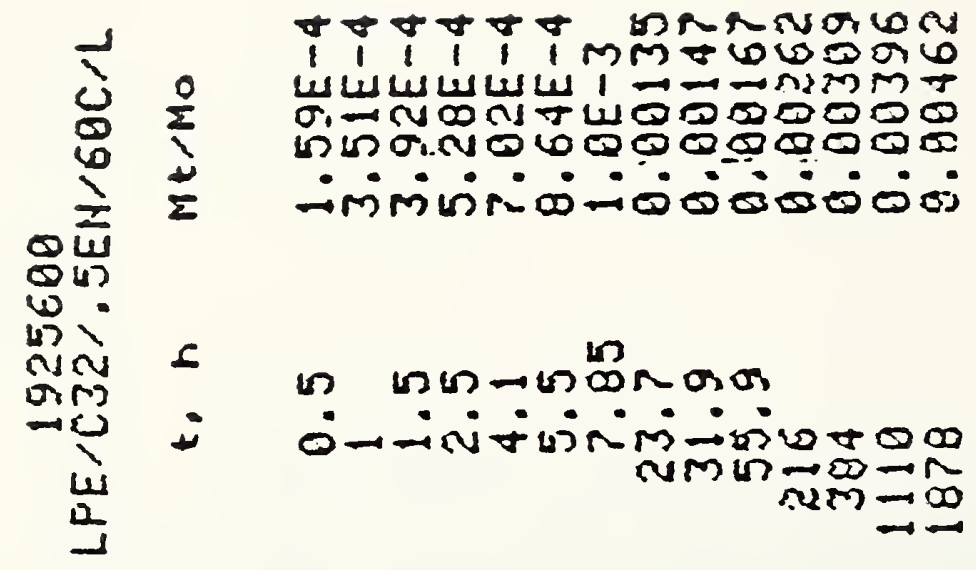
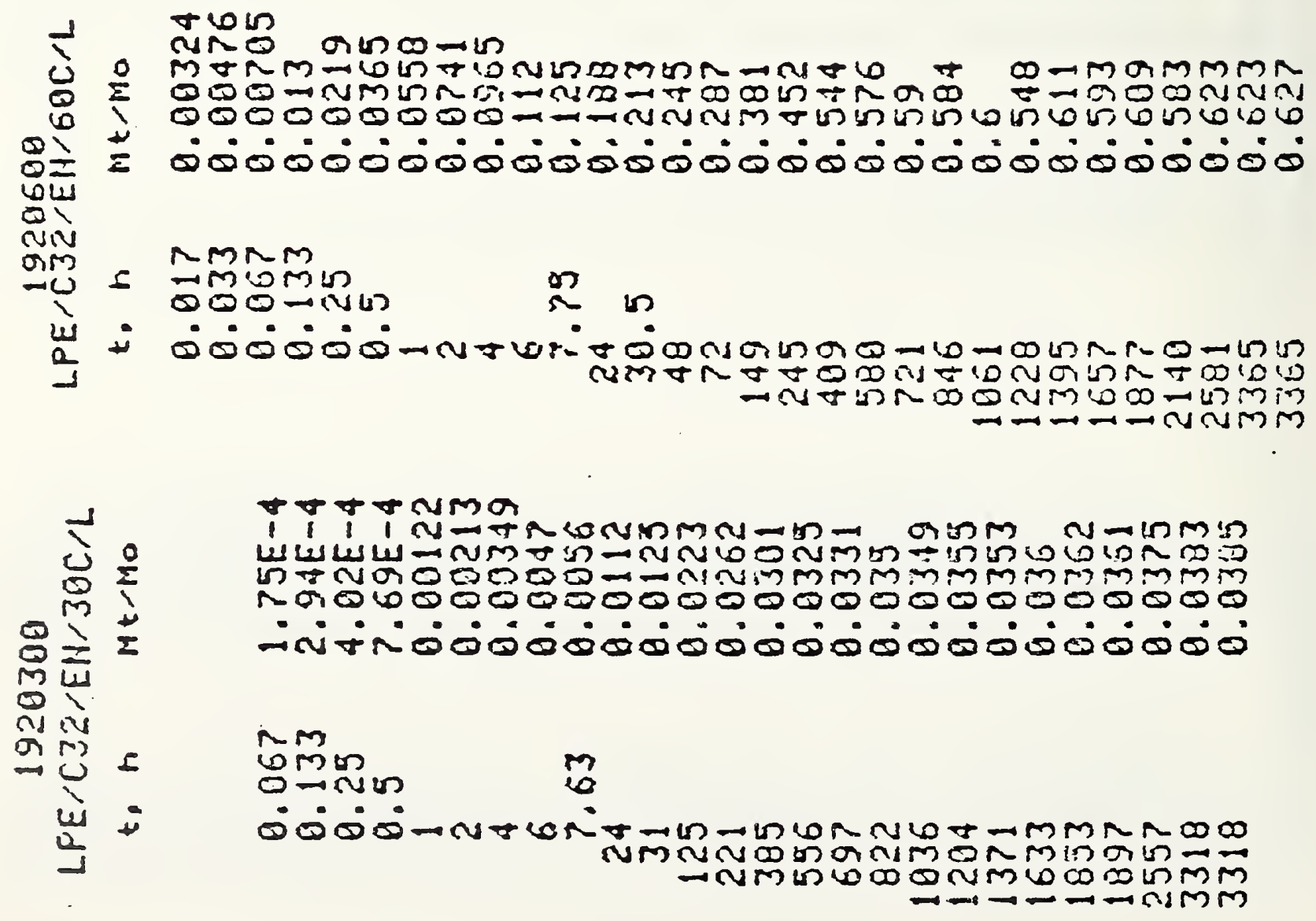


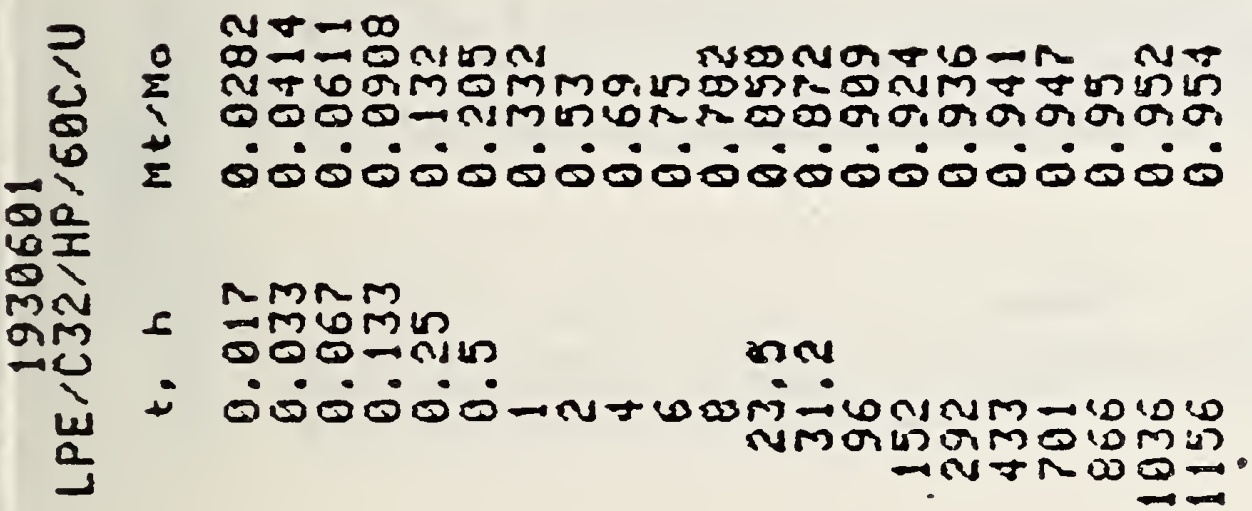

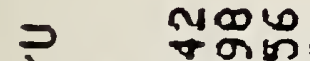
廿aña mán

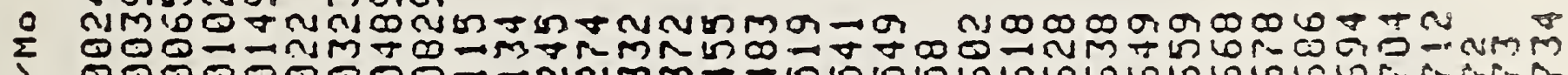

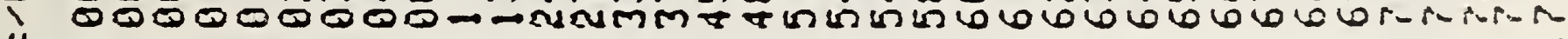

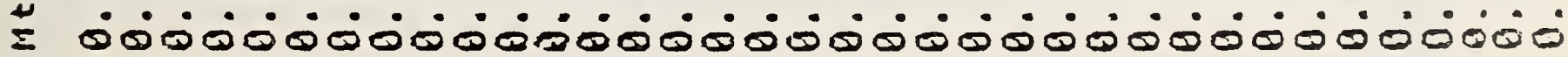

Nmm

$\rightarrow$ L 1 ming

-

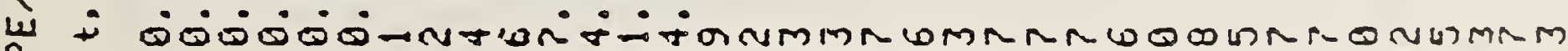
a NMUSR

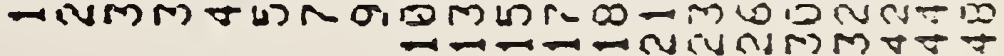



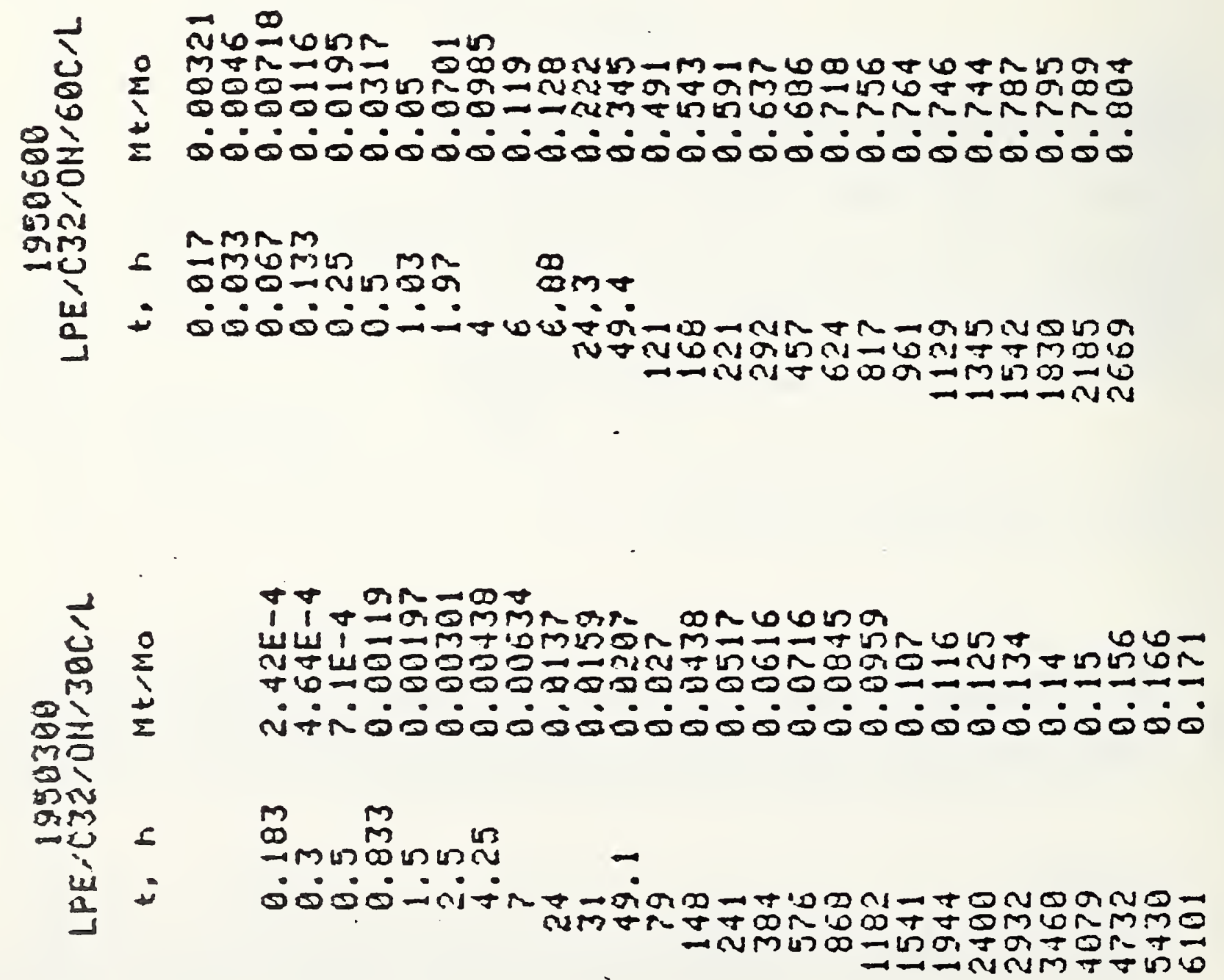


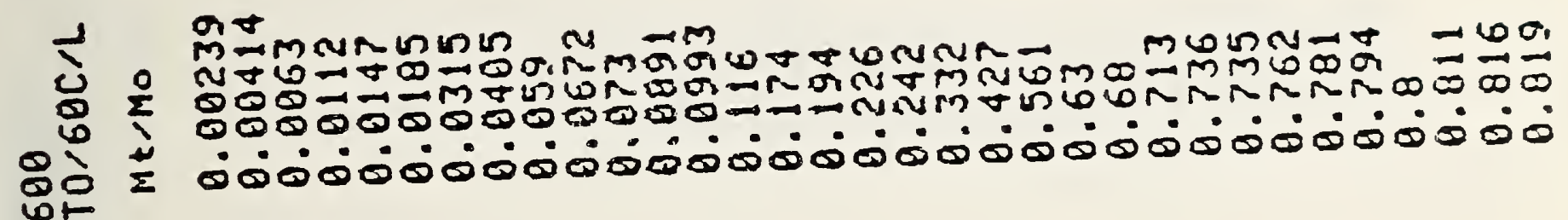

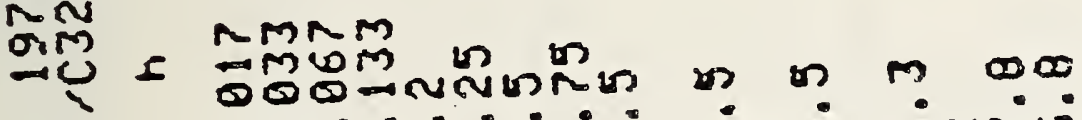

u :

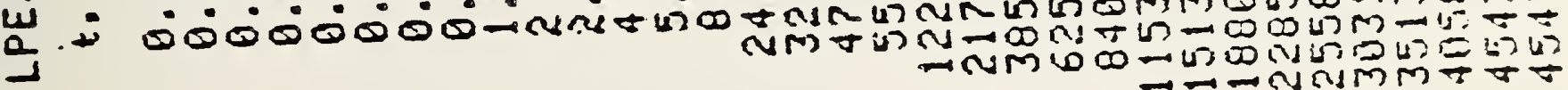

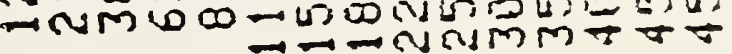

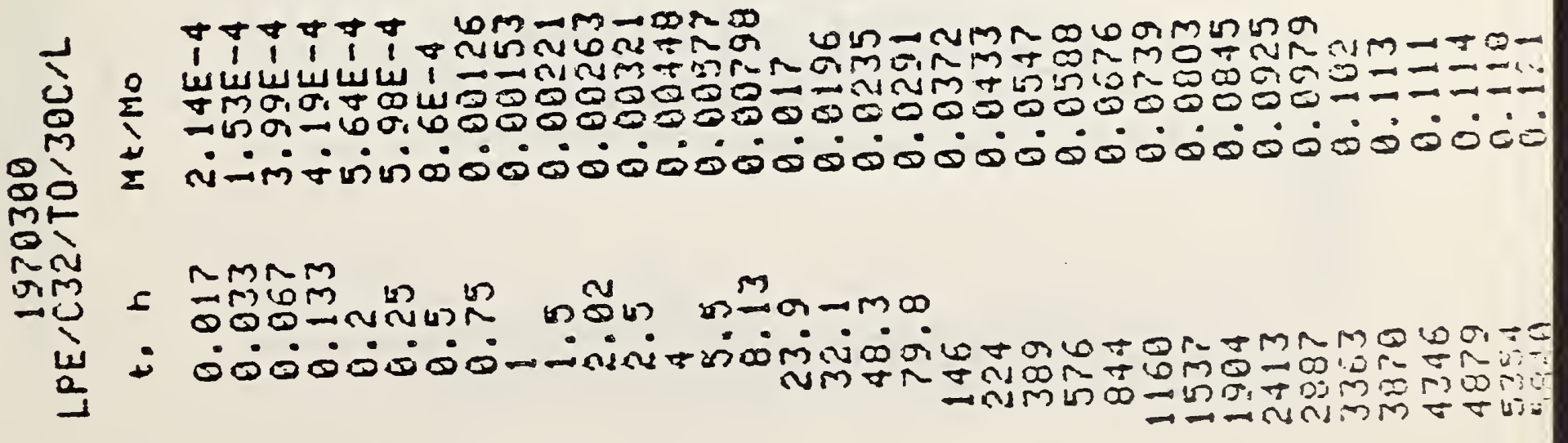




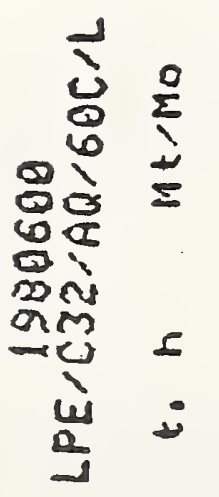

W

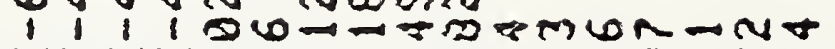

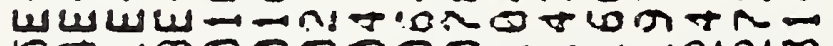

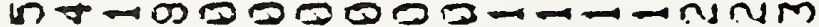

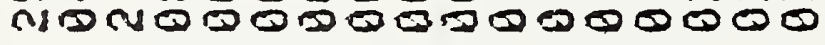

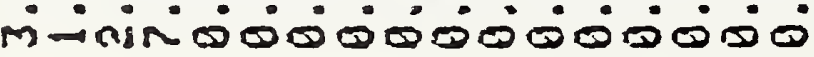

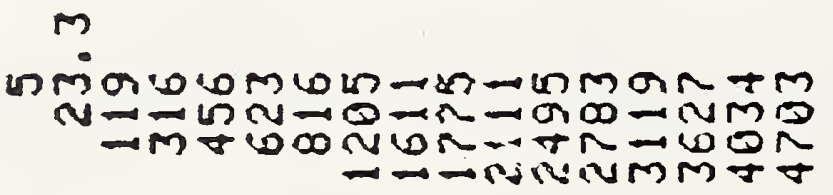




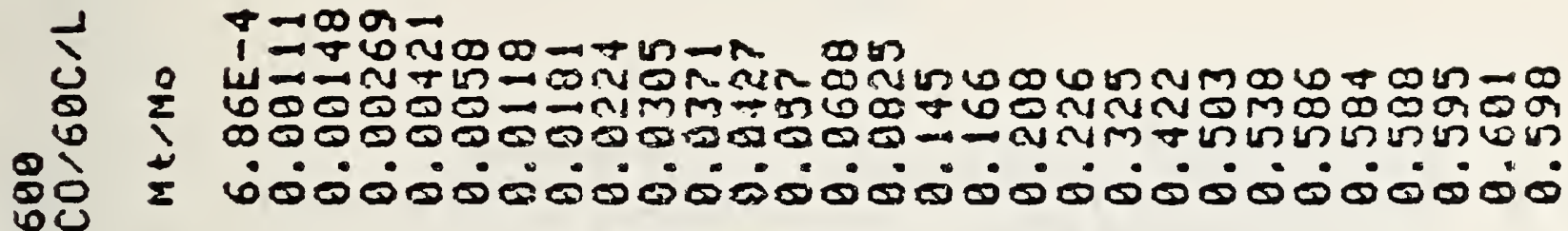

nmn $m$

-

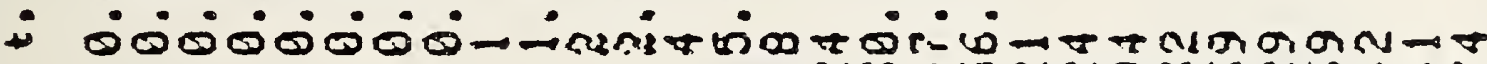
nm - ivmloontarin

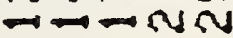

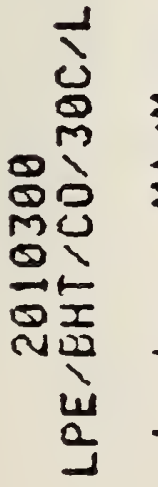

$+\$+\nabla+m+50 \omega 0$

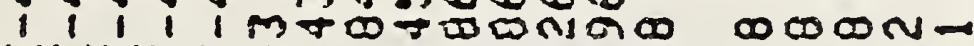

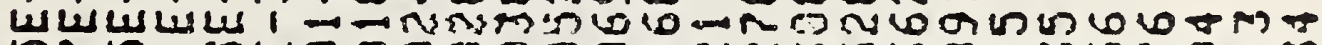

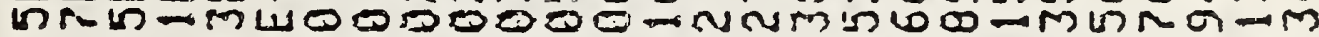

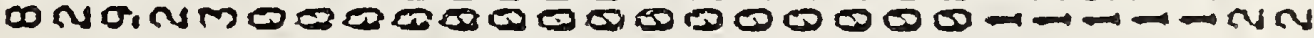
ن்

$n n m$

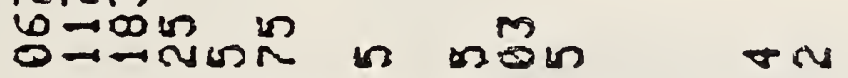

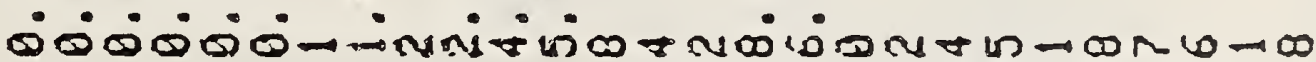
Nm

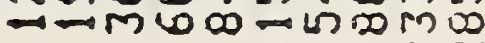




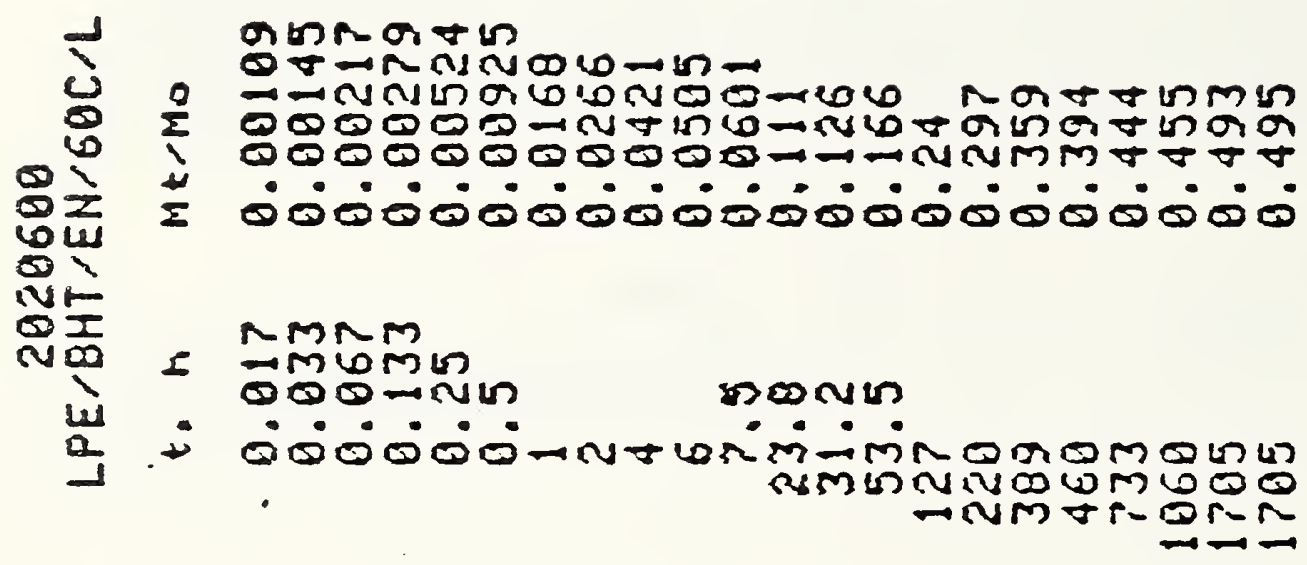

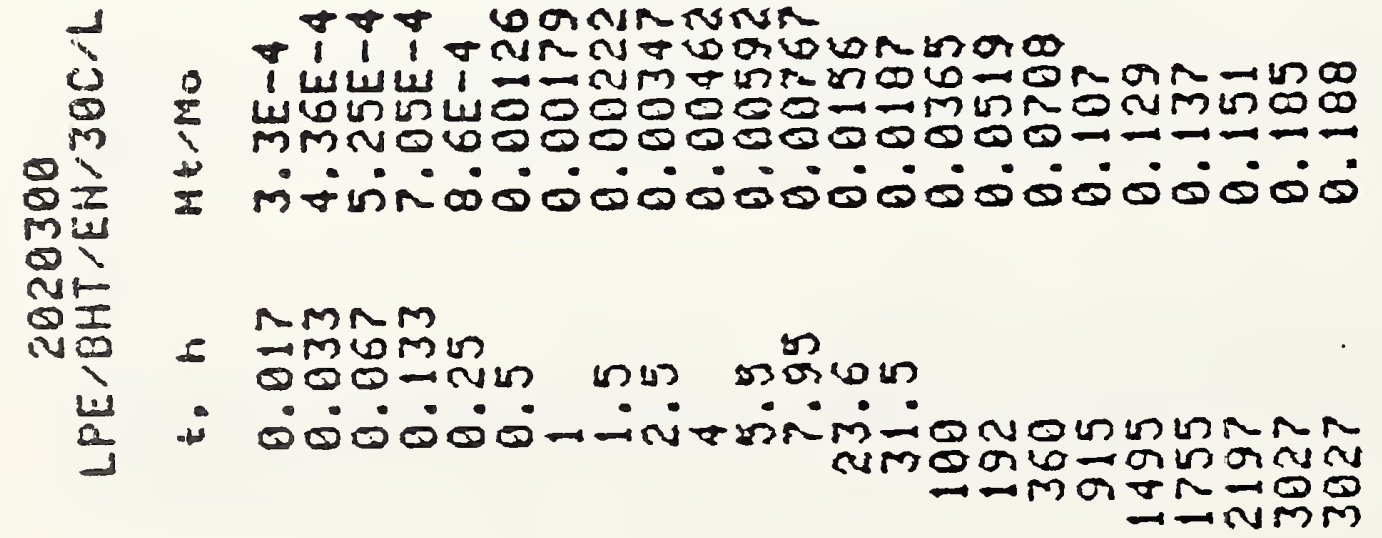




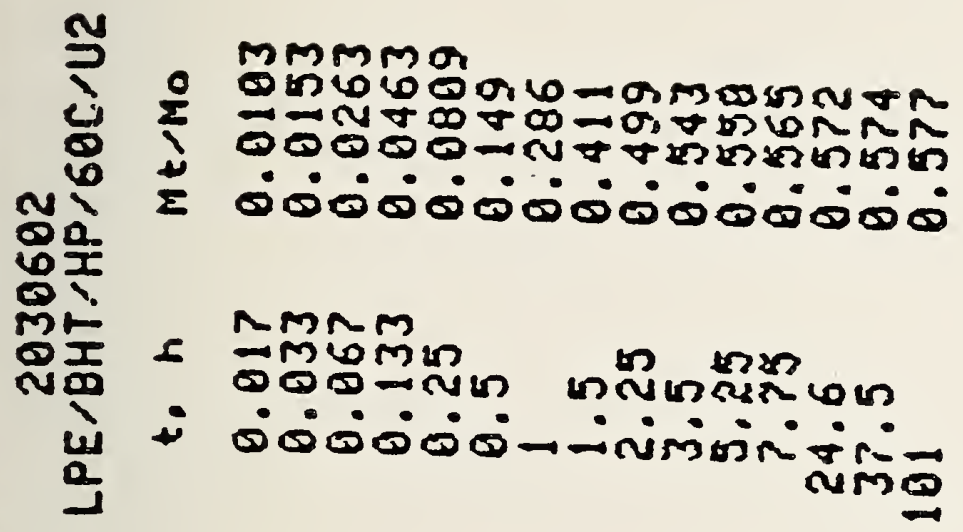

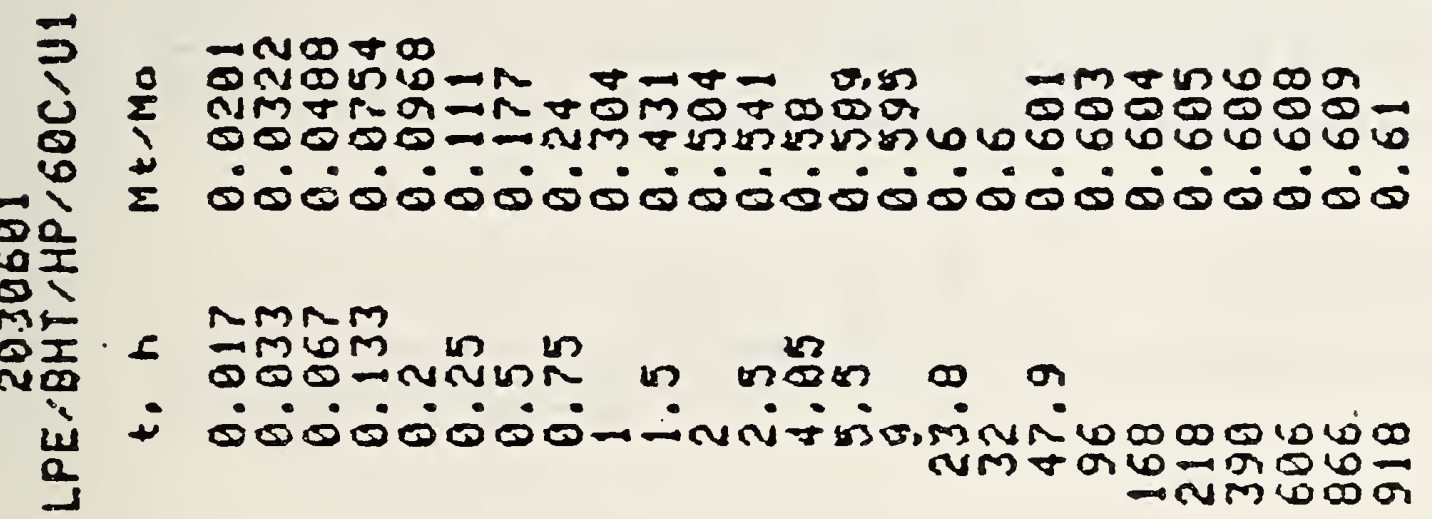

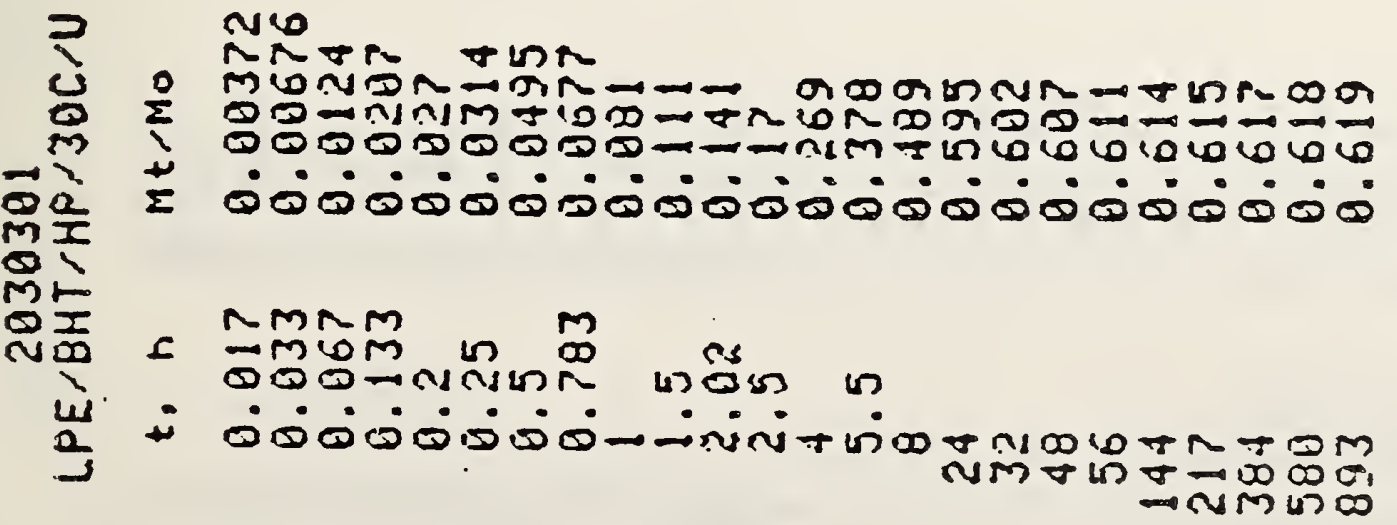




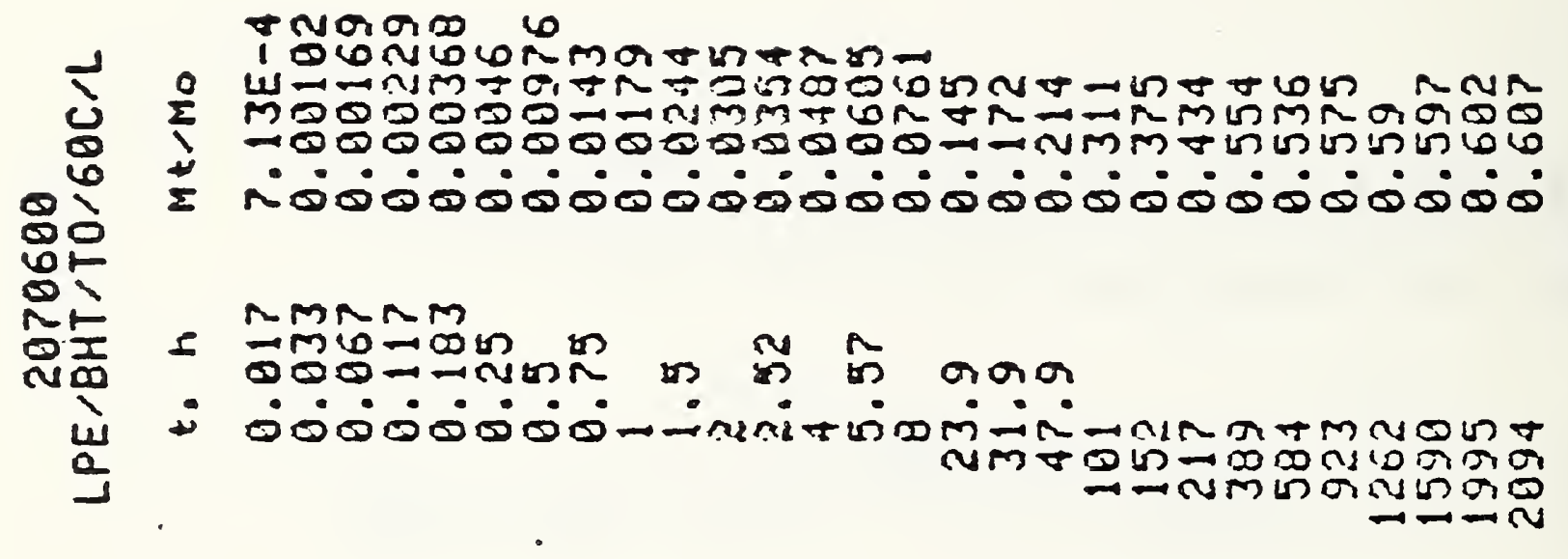

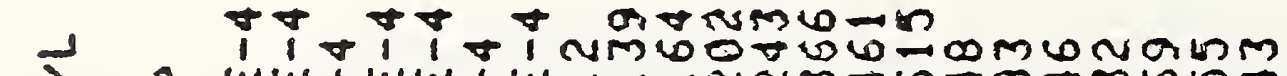

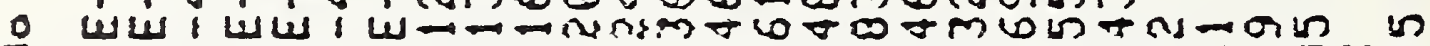

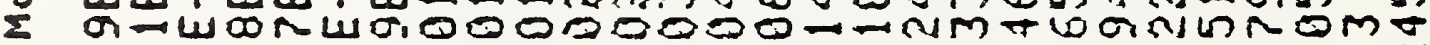

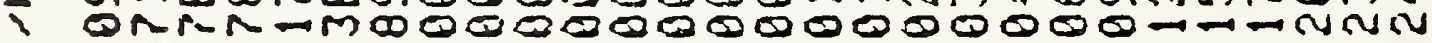

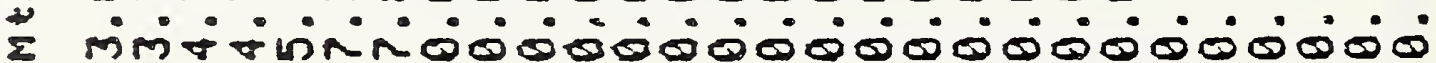
$\infty 0$
$m+$
$\infty$
Nr
QI $\&=m \in=\infty$

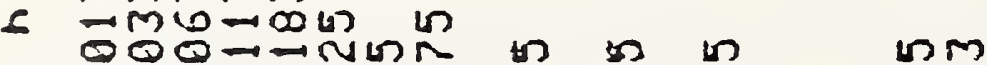
l.

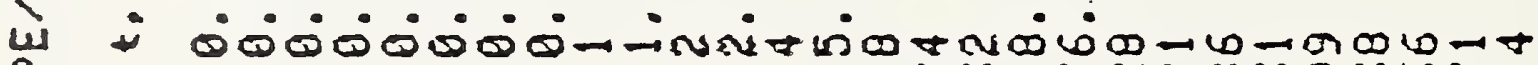
a

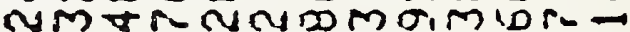

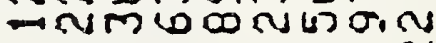




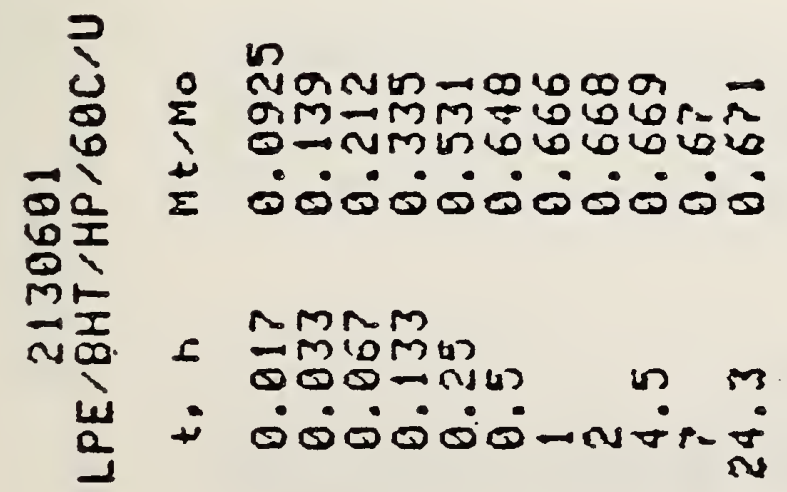




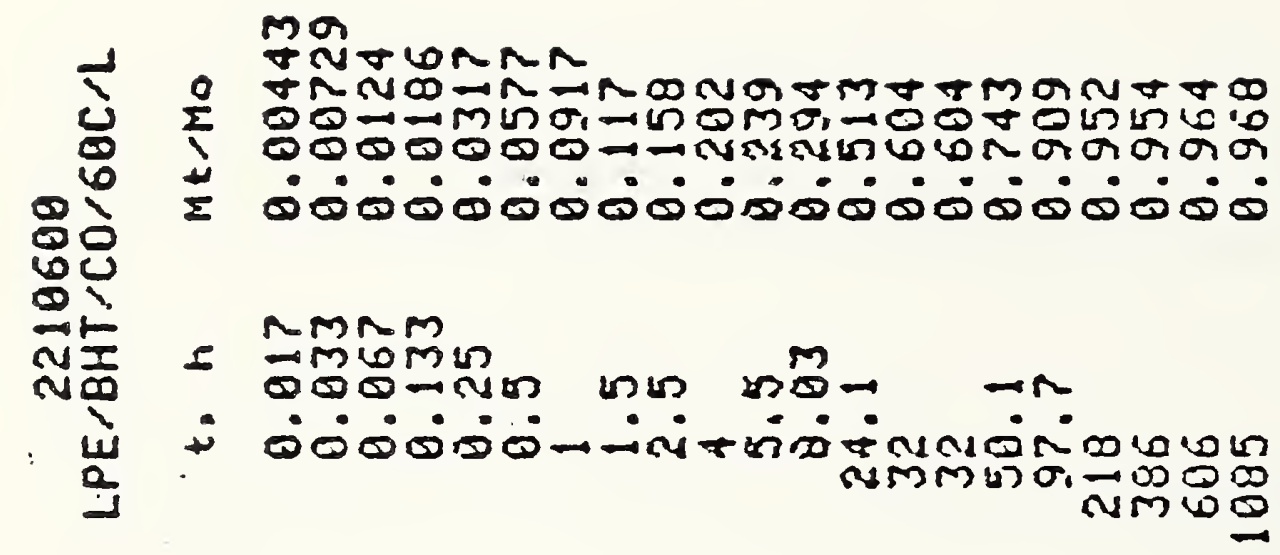

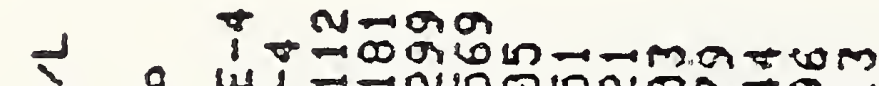

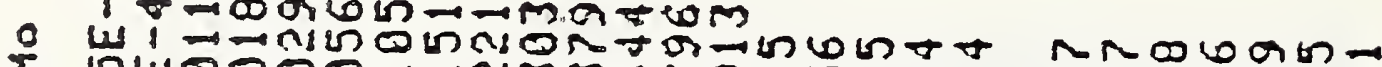

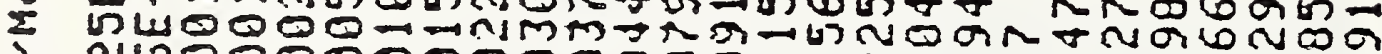

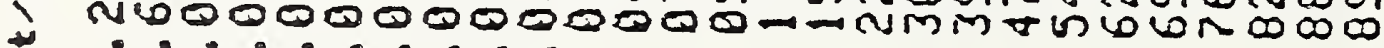

01

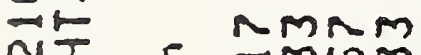

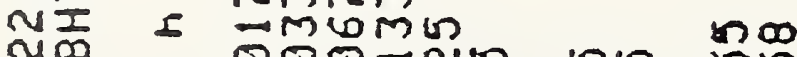

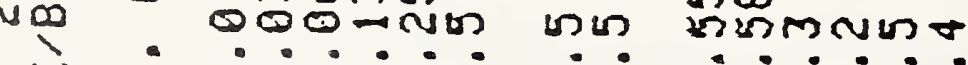

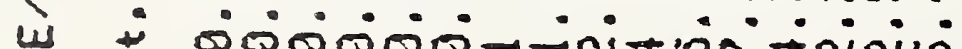

a
rum in a do $\rightarrow m \omega \infty=$ Un




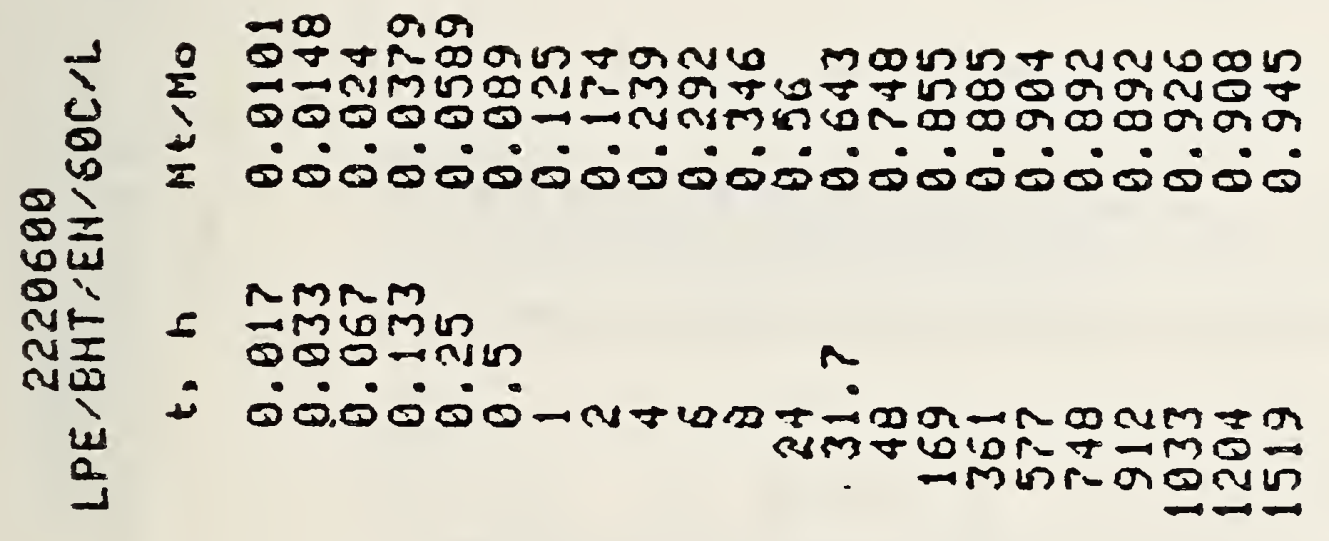

\footnotetext{
Nm-met

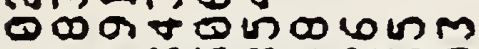

- - - -miñ

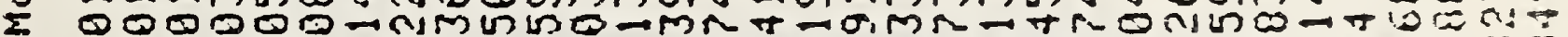

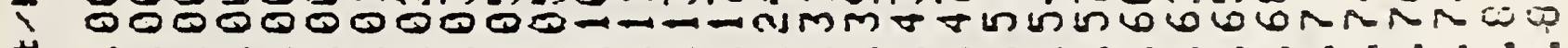

$\infty$

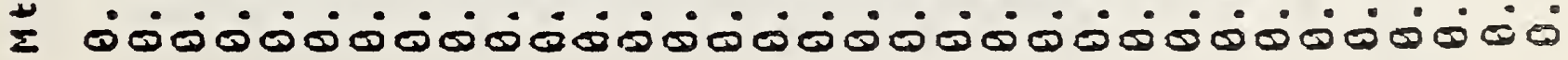

$0 z$

Mw

o)

nt

QU工

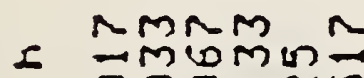

$000-\pi \operatorname{rn}$

$\ln$ or

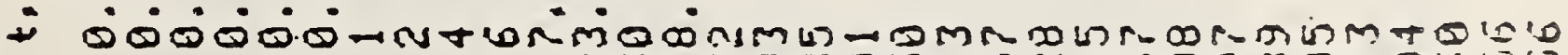
NM

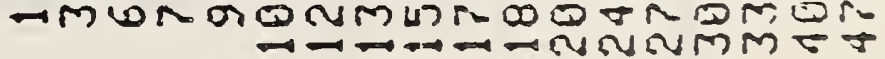




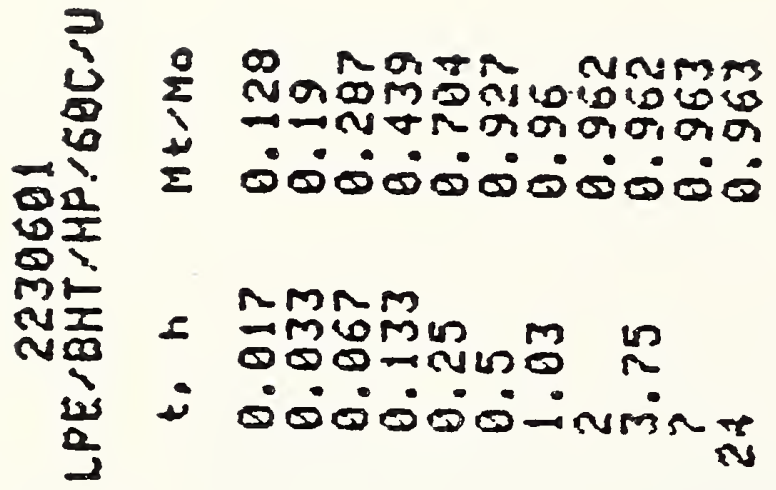

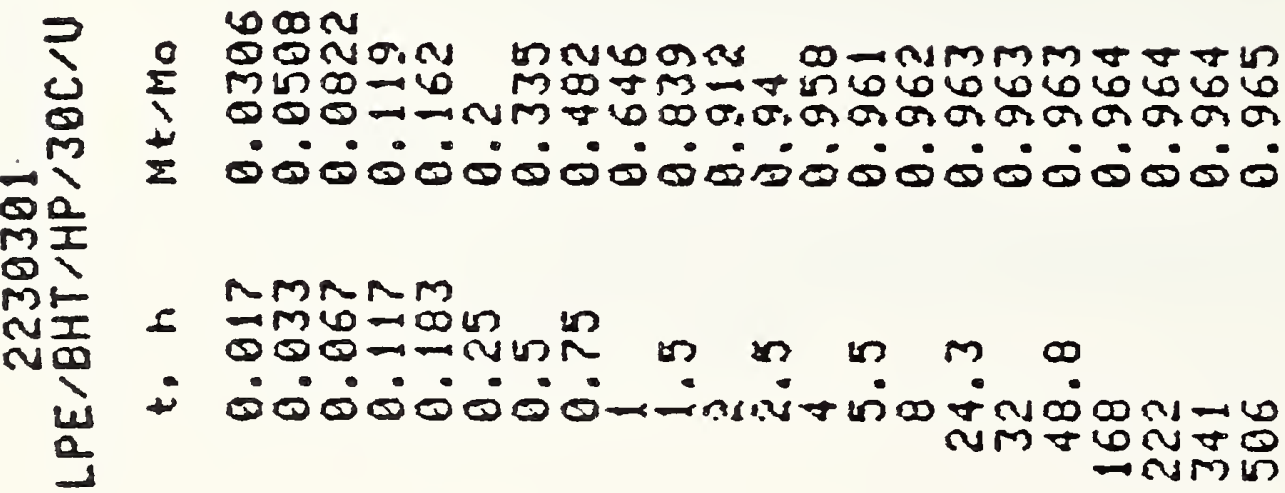

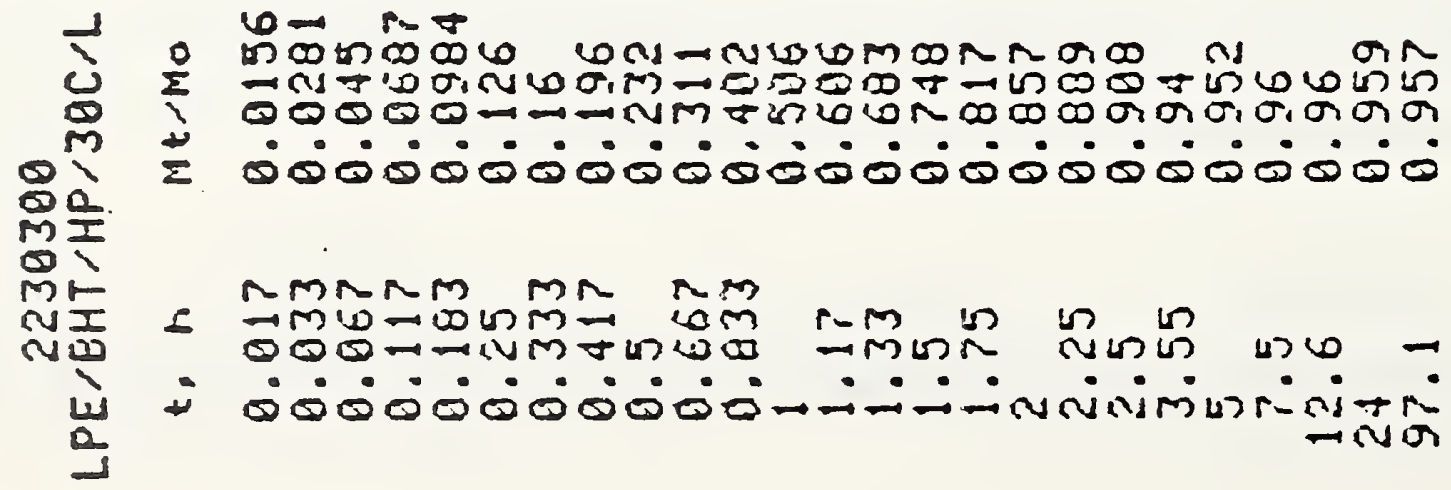


Non

NoN $\rightarrow \forall+\infty$

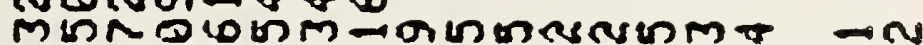

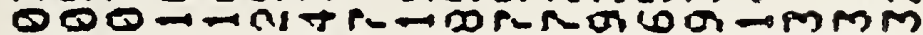

00000000- InM T-

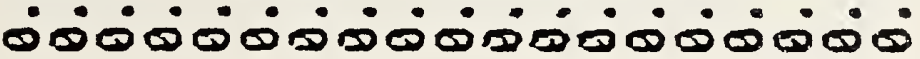

NMNRM

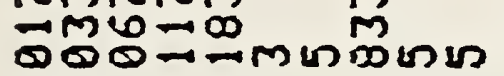
هंவ் $\ln _{2}$

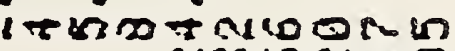
NMU

$m=0 . a$

कaw NMUDMONRR-GBNR-

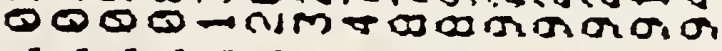

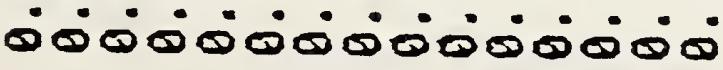

$\sim m$

Cmun

o-nis bant

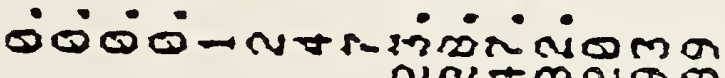

तre $+\infty$ Non

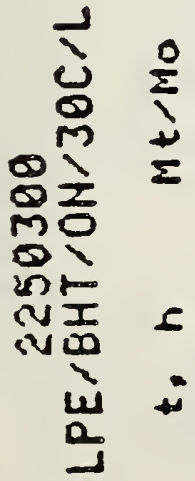

\section{wing}

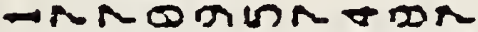

-

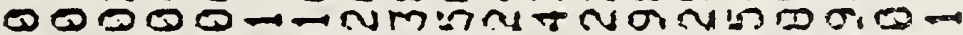

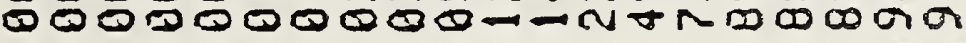

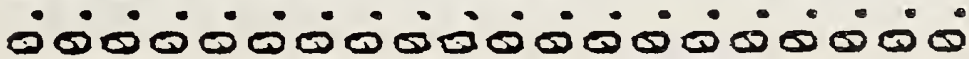

$\sim \sim m$

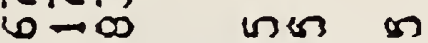

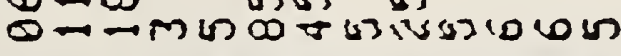

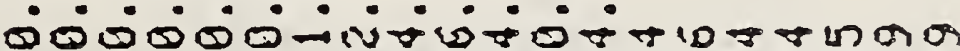

nimun $=0$ Nin os

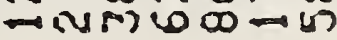




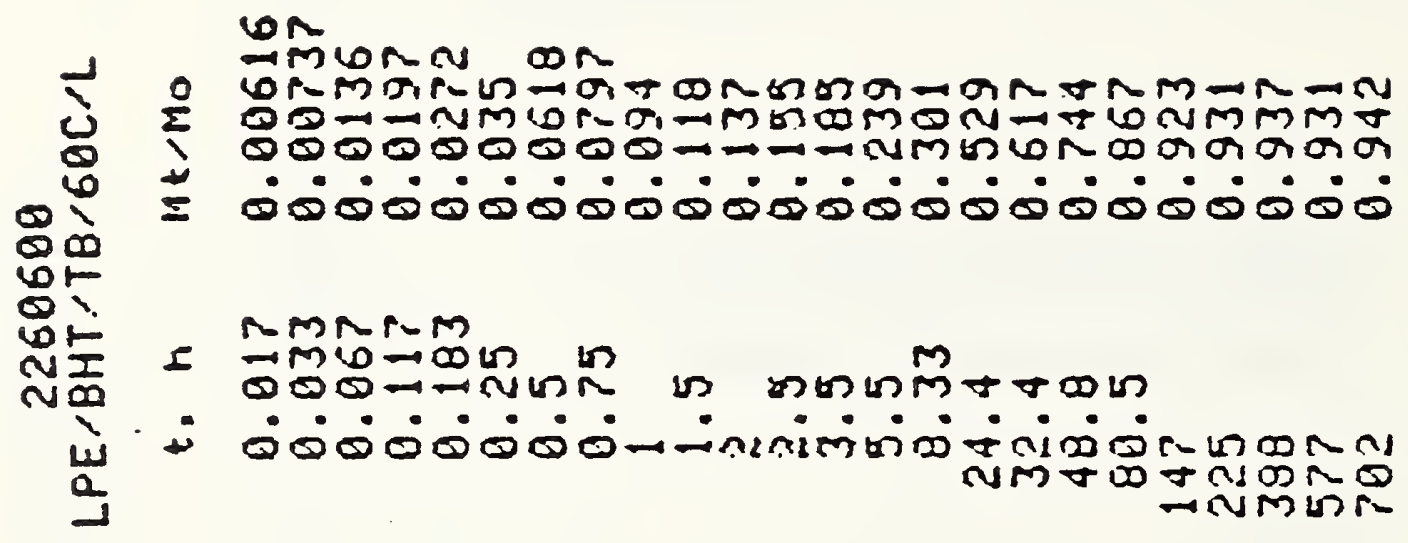

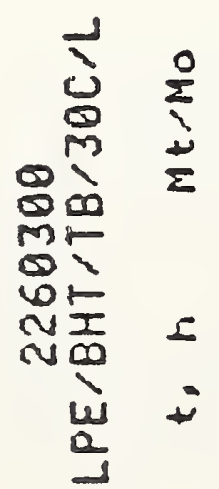

$\rightarrow \cos$ in

$\infty=\ln 0 \mathrm{mNm}, 000 \mathrm{~m}$

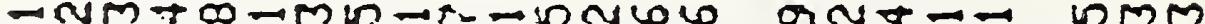

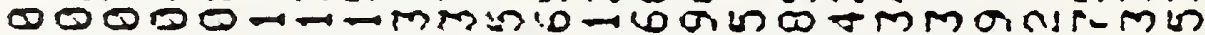

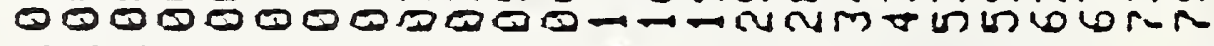

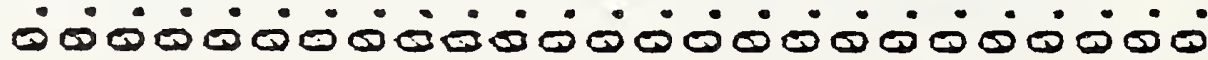

m

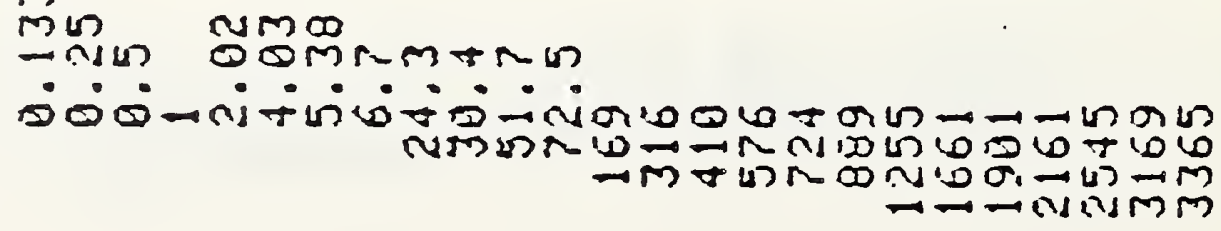




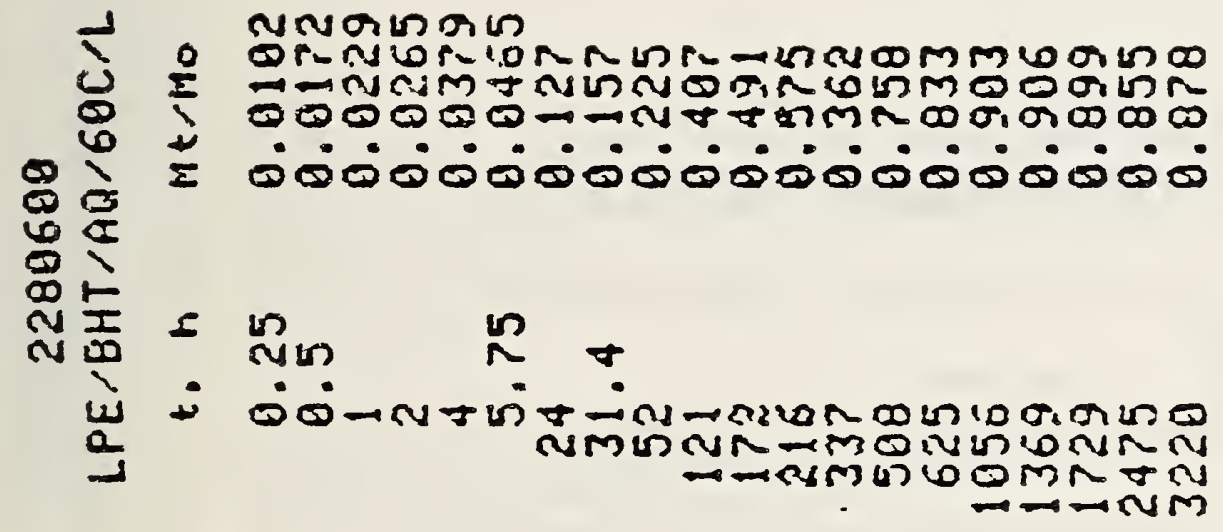

\footnotetext{
$\pi-\pi \sin$

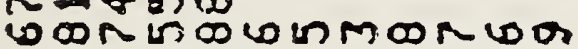

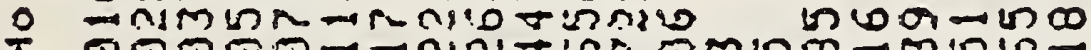

I

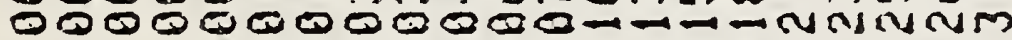

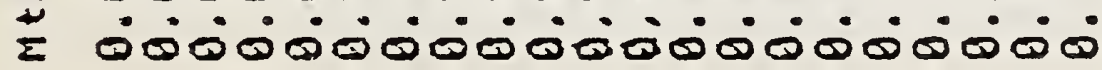

00

$m \Phi$

舟

$\infty$

तथ

Inis nom nom

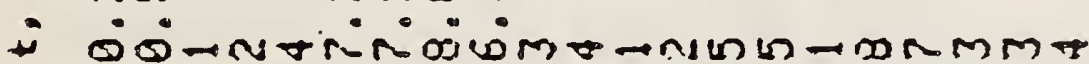

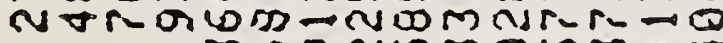
-
} 


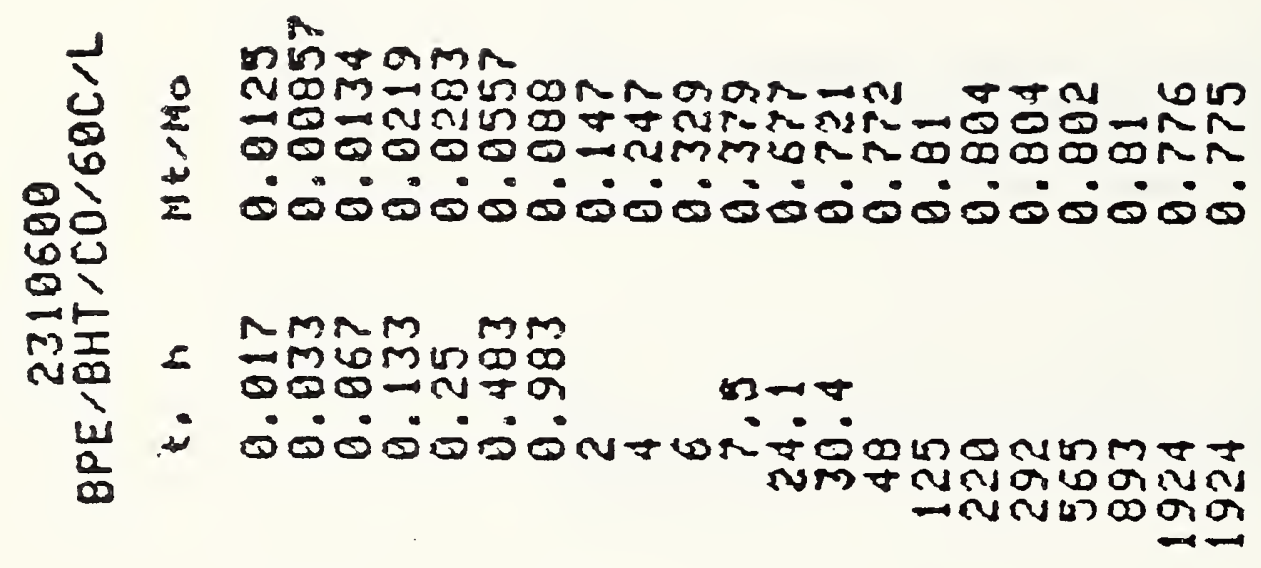

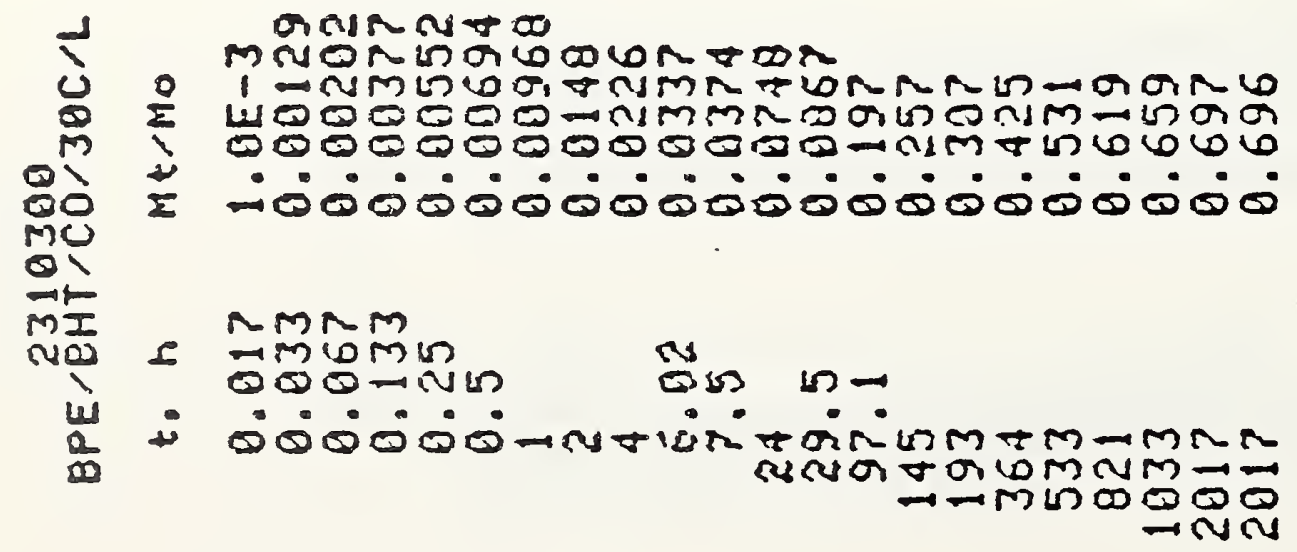




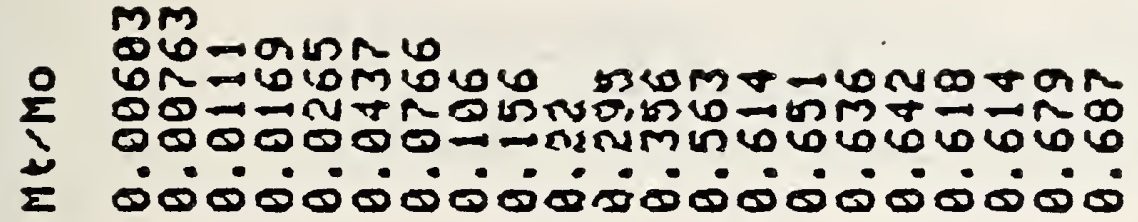

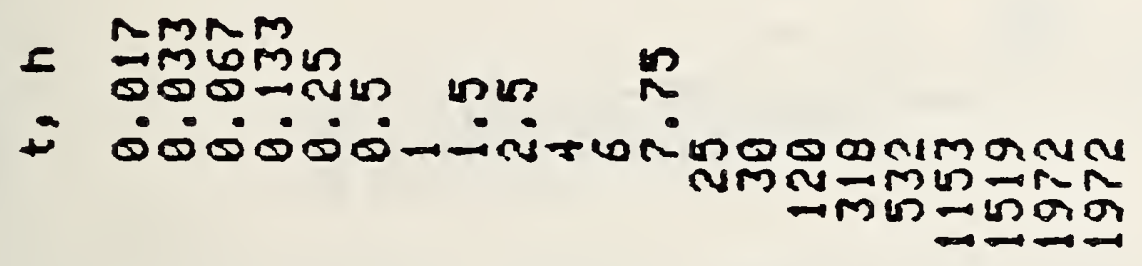

\section{an}

Nmenimor

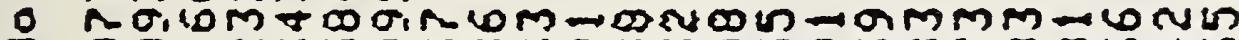

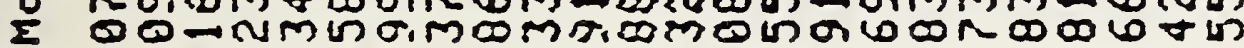
$0000000 \Rightarrow$ - MUM

=

DI

nta

MI

Nos

in

in

- $\min m$

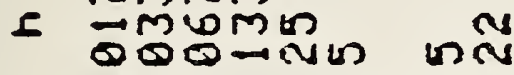

UMज.

+ Nonging

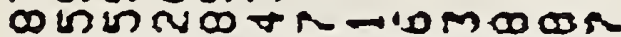

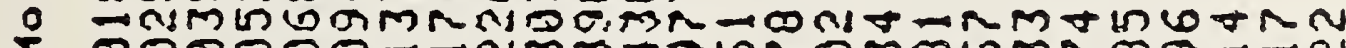

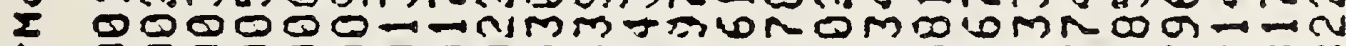

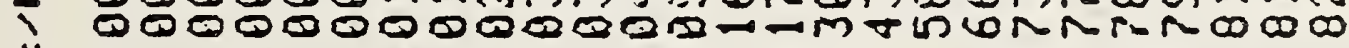

01

$m I$

लำ

rmam

e $=m 6 m i n$

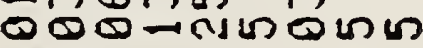

L 


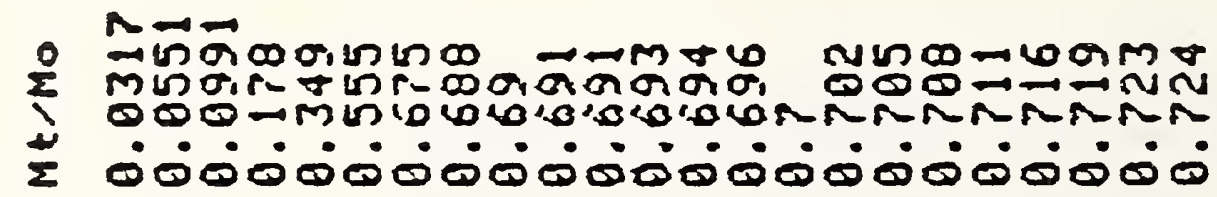

m)

62

$\infty$

$M$

vion

is

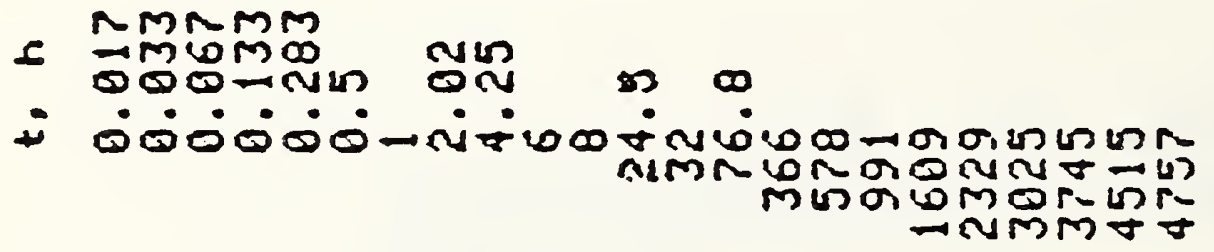

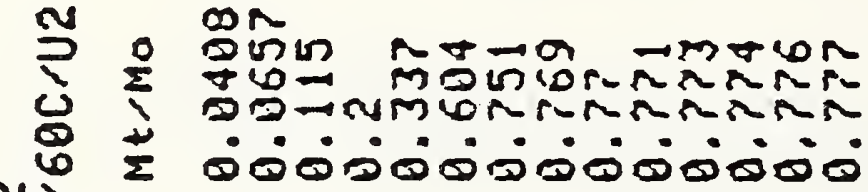

N)

$\infty$

$0 \div$

MI $\&$ ATOMU

as

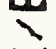

us

$\frac{2}{\infty}$

* க்

os $\rightarrow$ त

3

$\rightarrow 1$

$6 \frac{2}{1}$

$\infty$

$m+$

$m I$

No

U.

$\frac{11}{a}$

$\infty \infty$

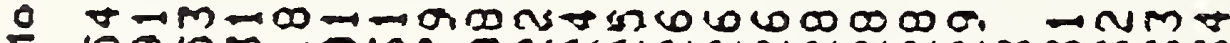

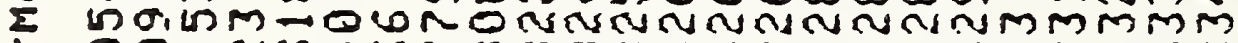

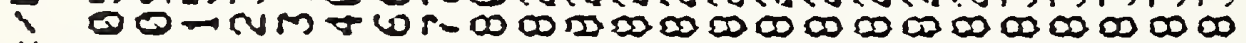

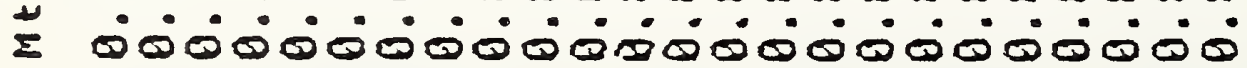

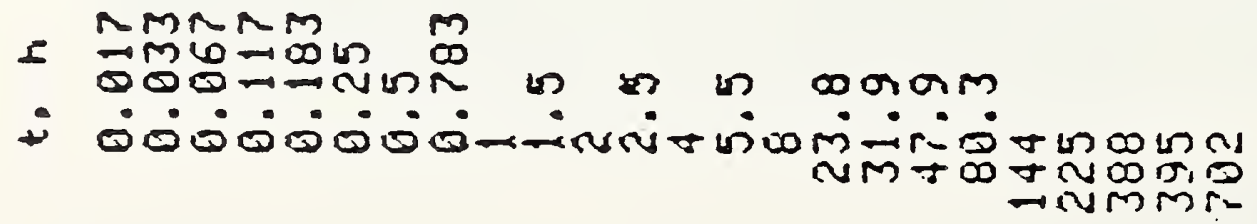

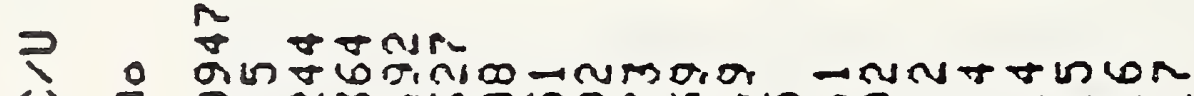

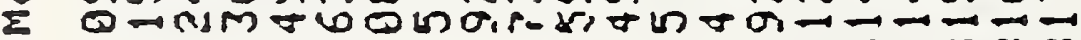

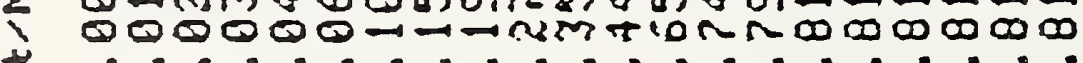

+

$\overrightarrow{0}=000000000000000000000$

ma

0 '

$M+n m n R$

$M I E N M \omega \infty$ in

No

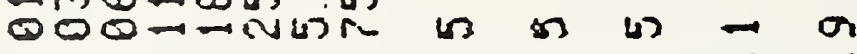

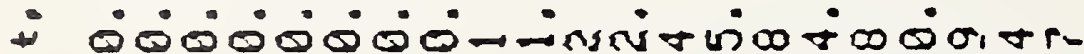

u.

$\infty$

N $=\infty \frac{\infty}{N}$ in 


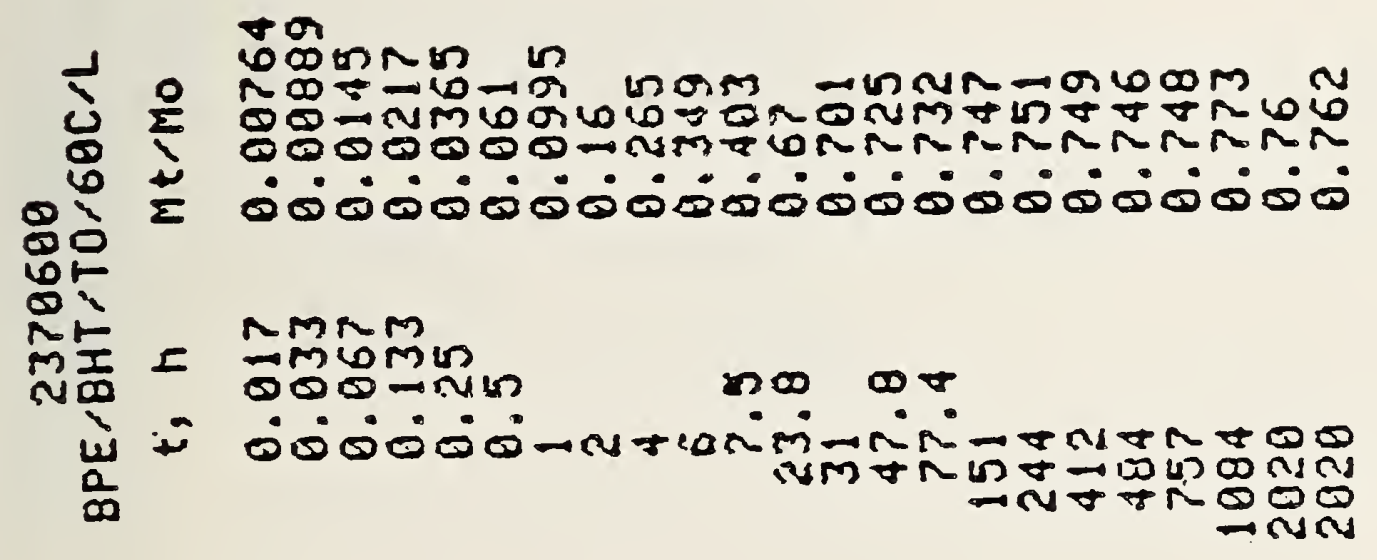

\footnotetext{
M $\infty \pi-\cos$

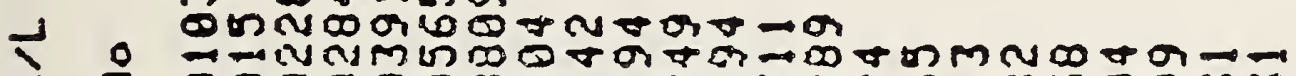

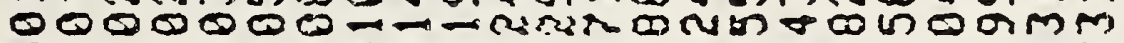
M L

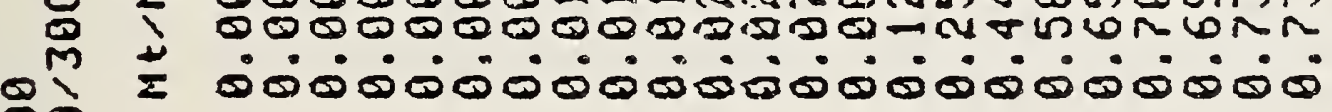
은 Di

NL $\quad N M N m$

MI $E$ -

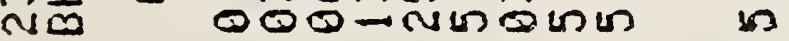

iो + $\dot{000000}$ -

$\frac{a}{\infty}$ 


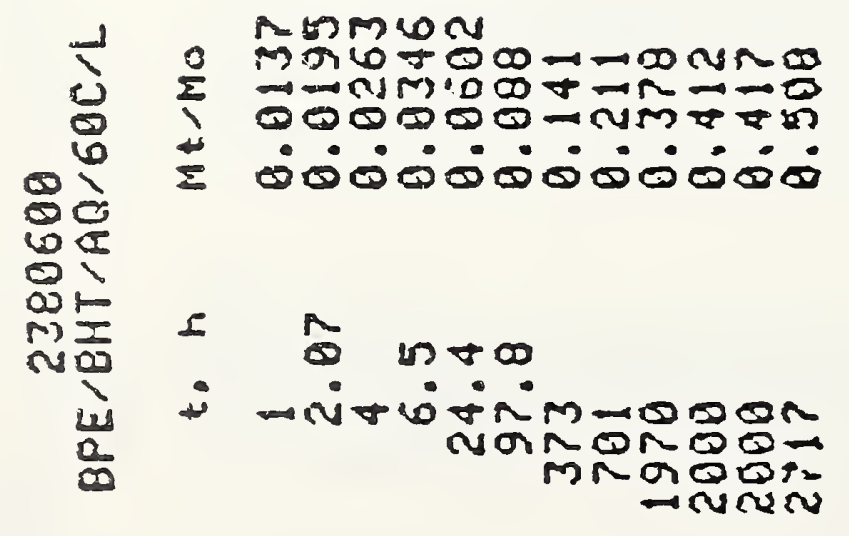




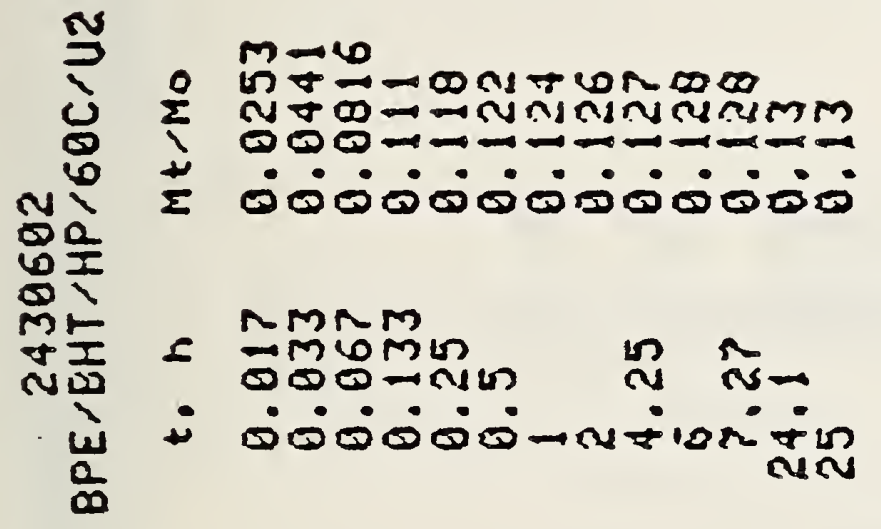

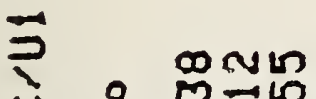

- 0 muntranconan-

U इ

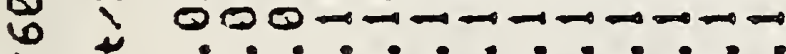

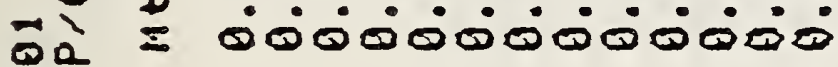

6 중

01

$m$

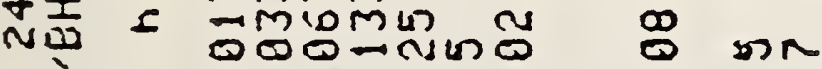

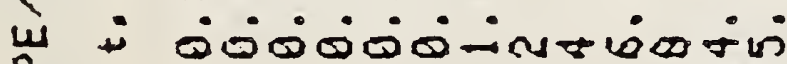

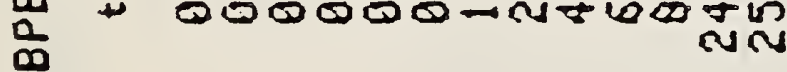



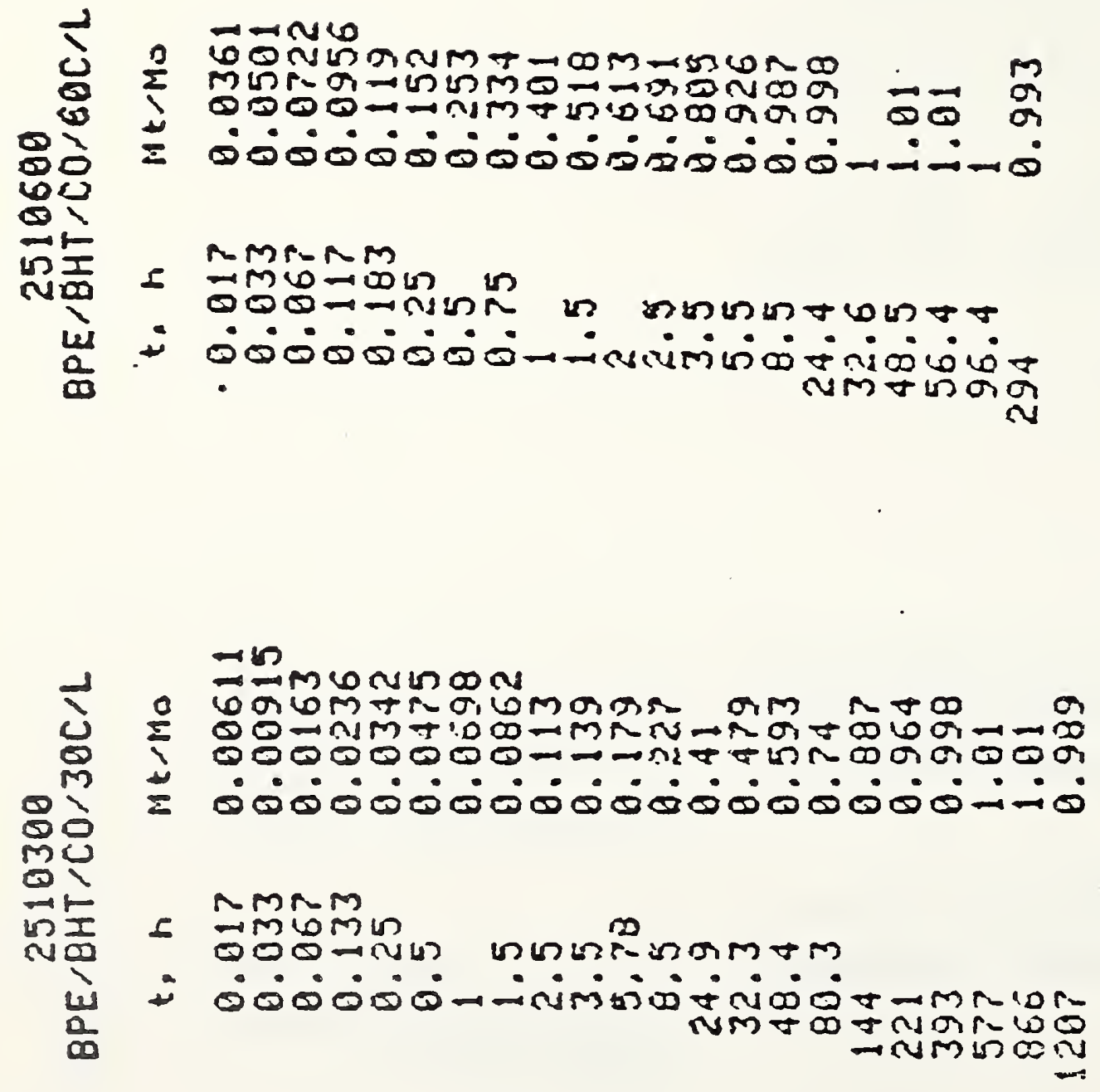


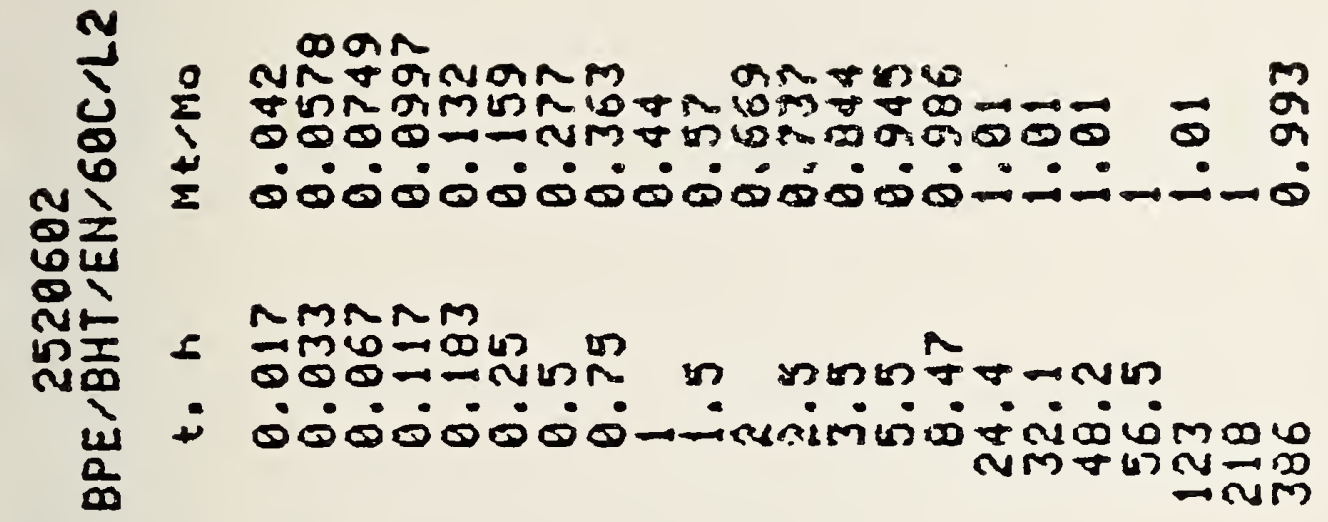

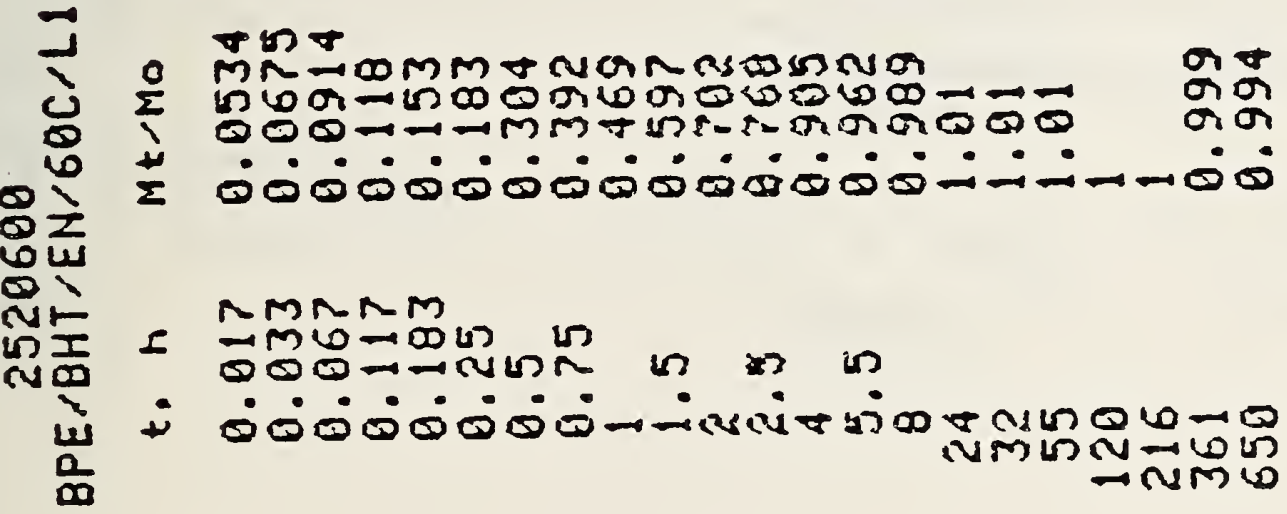

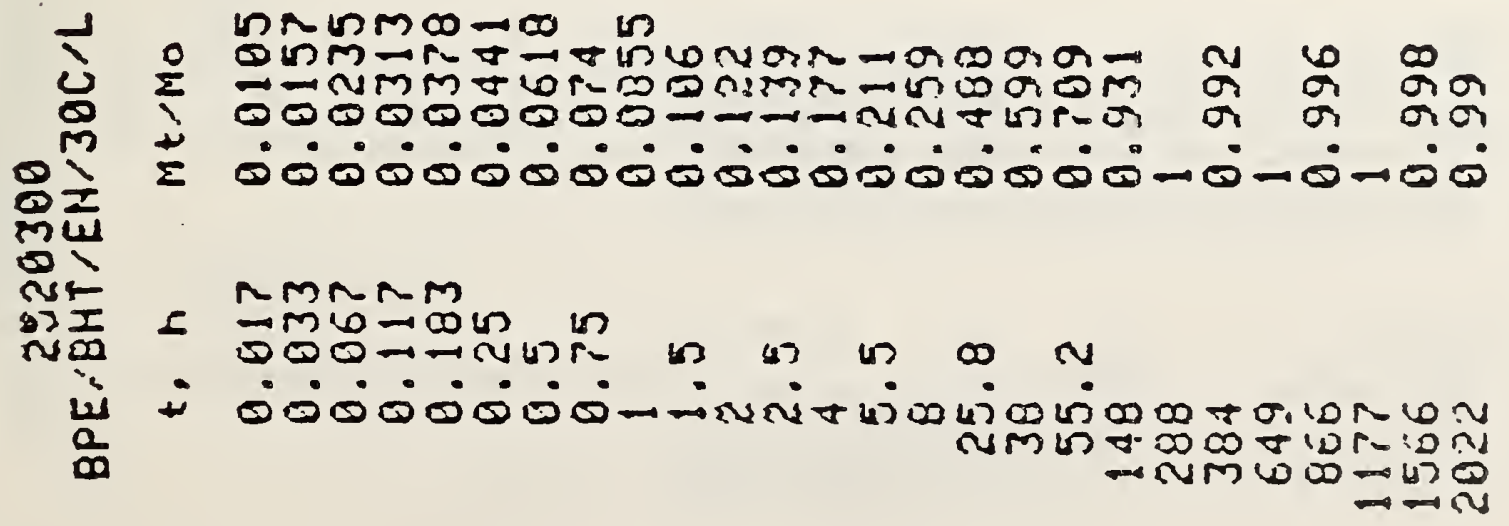



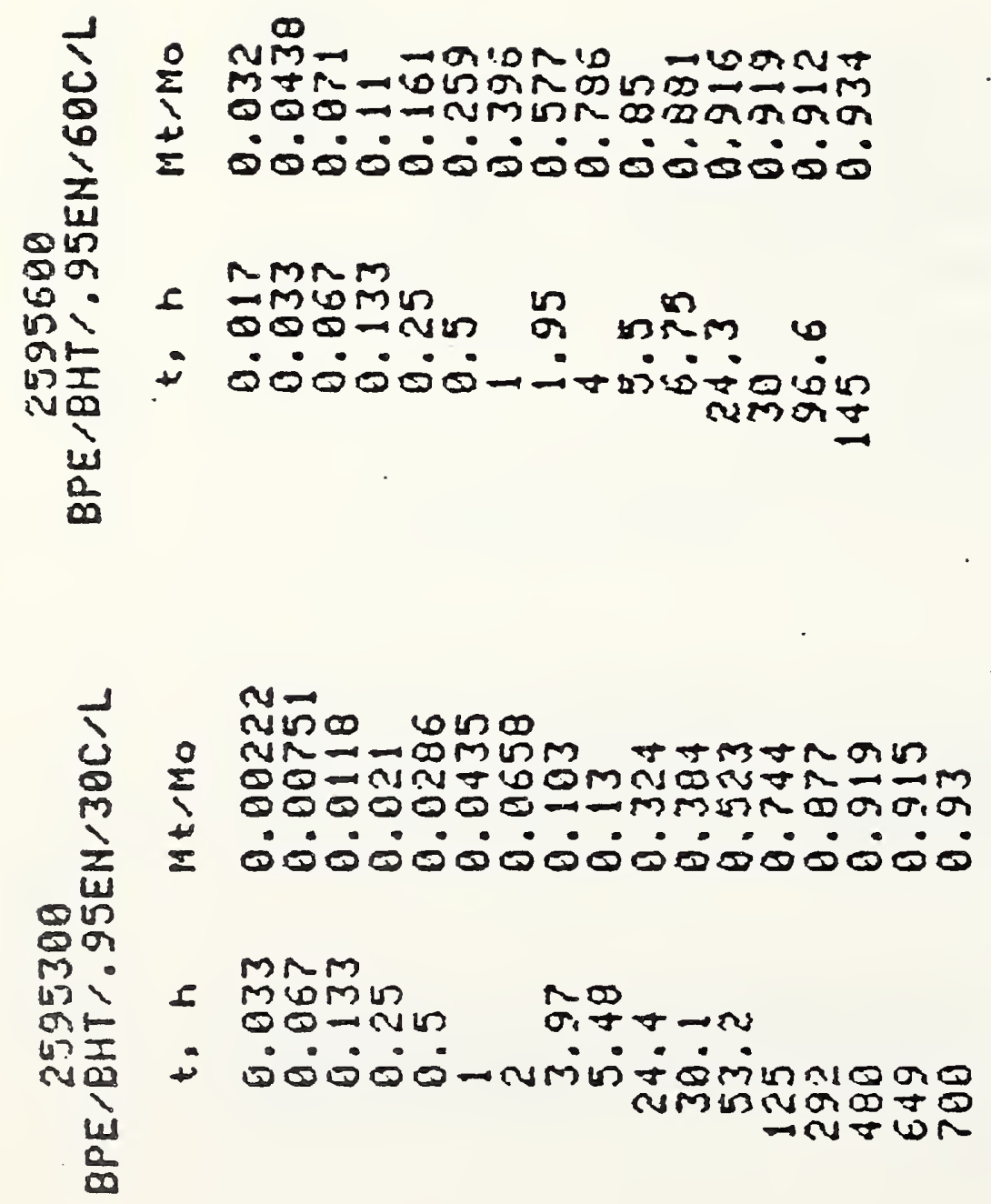

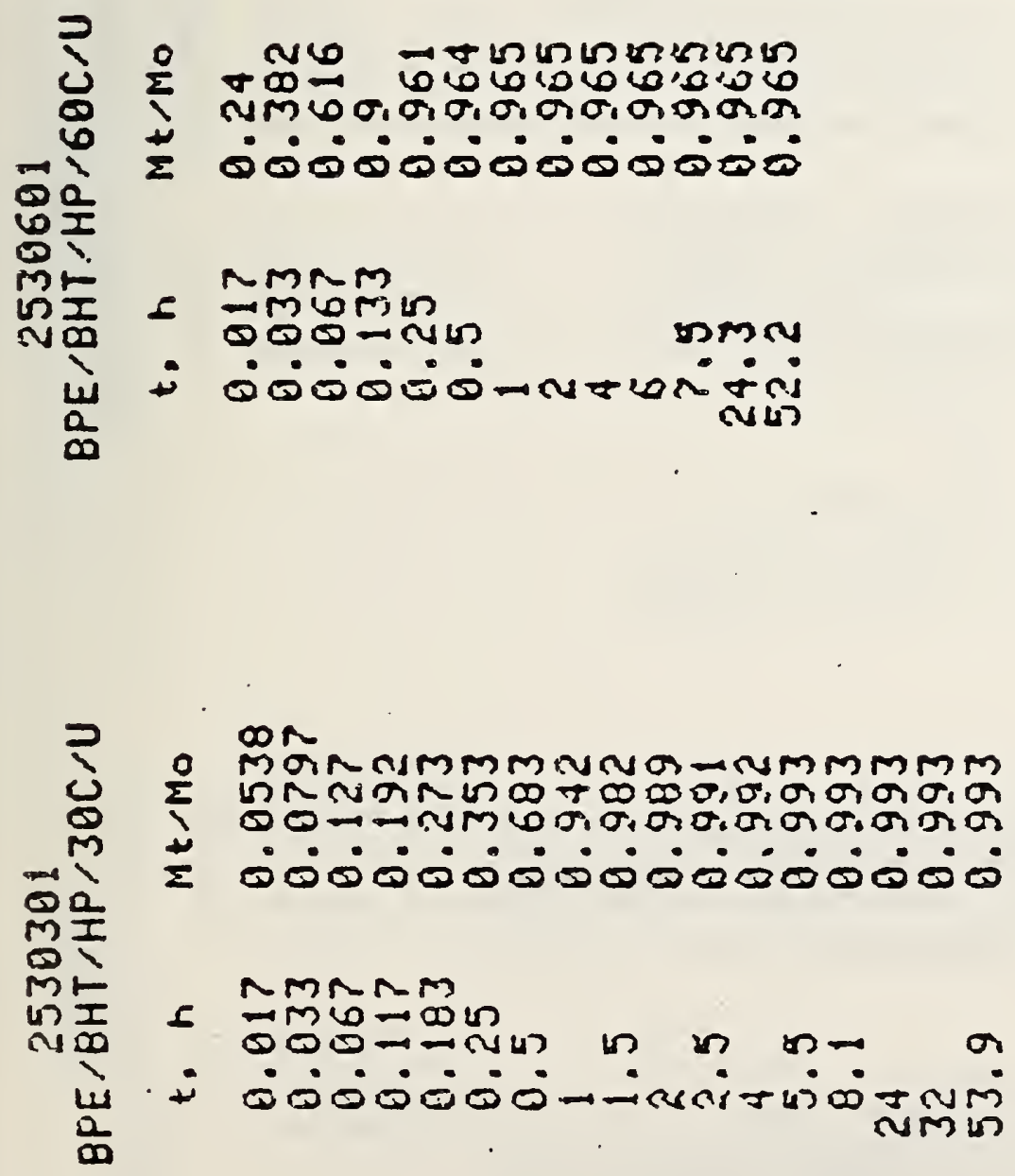


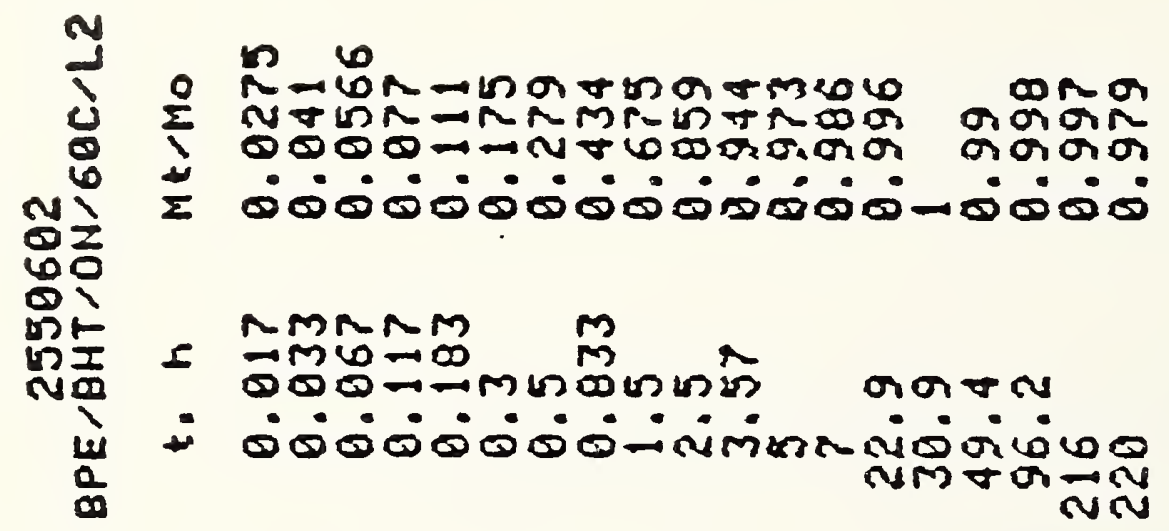

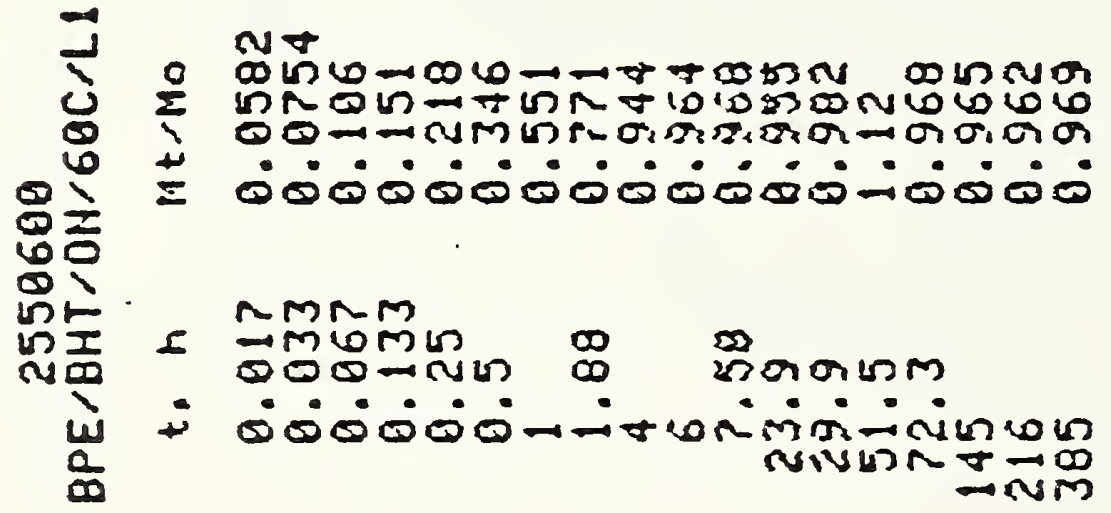

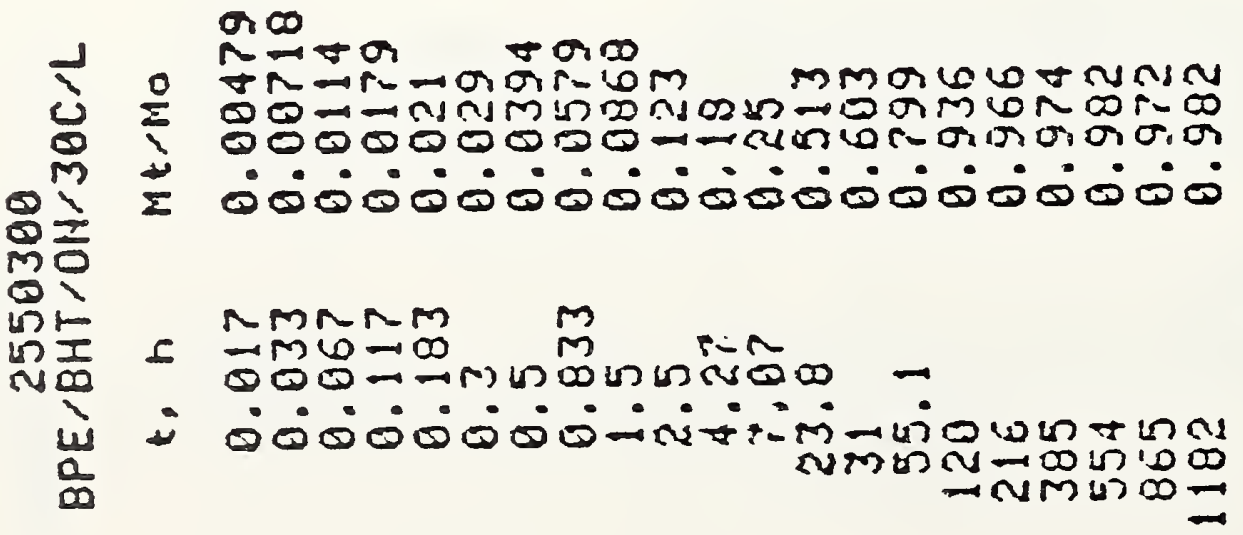




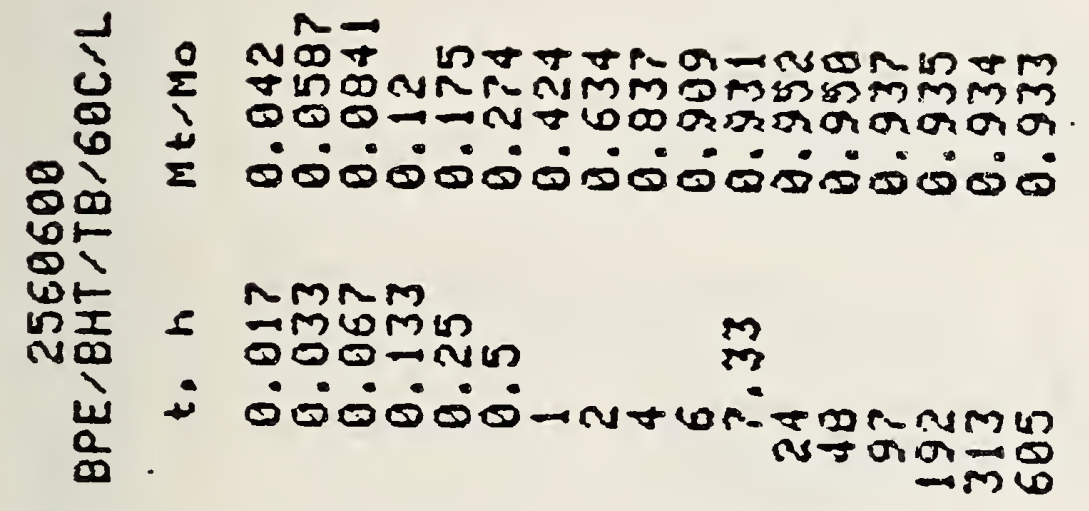

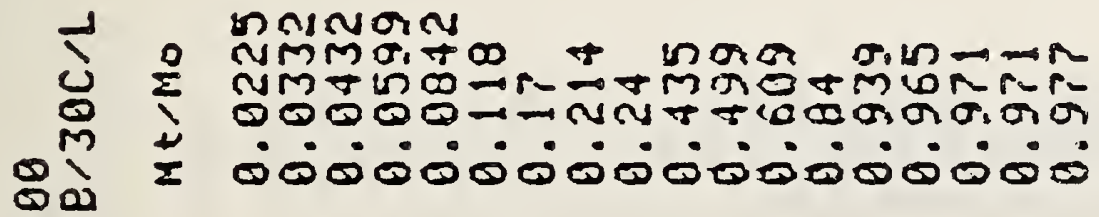

M

M-

$\omega$

I $=m$

UI I DMU

D-nin

से ثंक्षं०

4

$\frac{1}{\infty}$

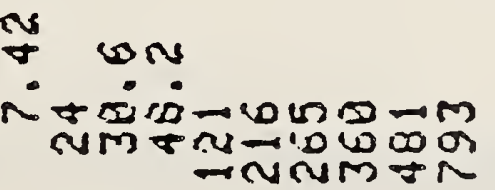




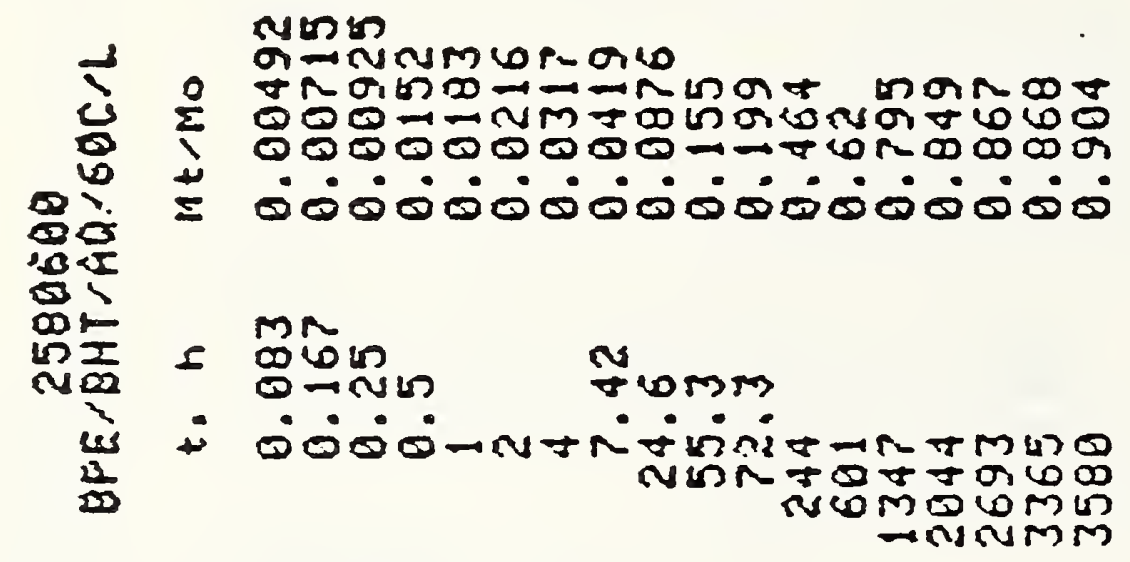

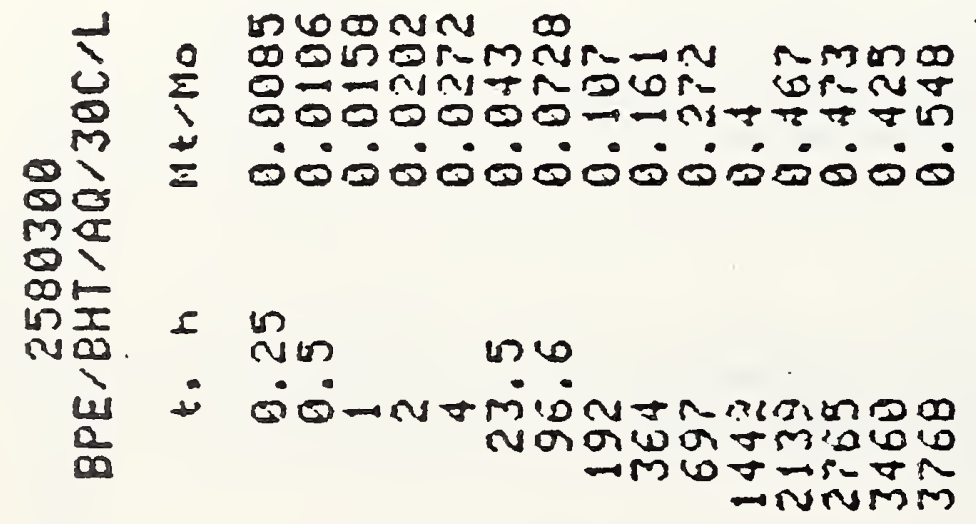




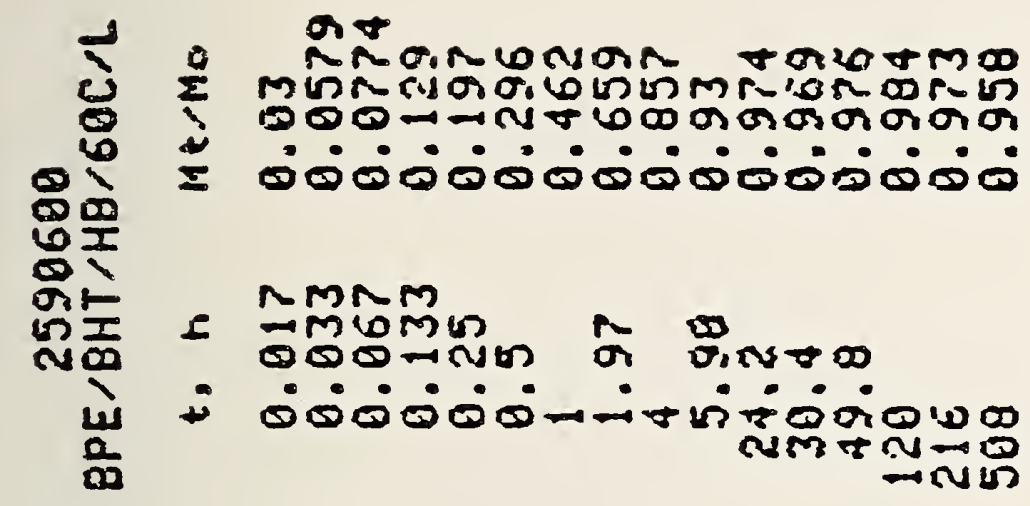

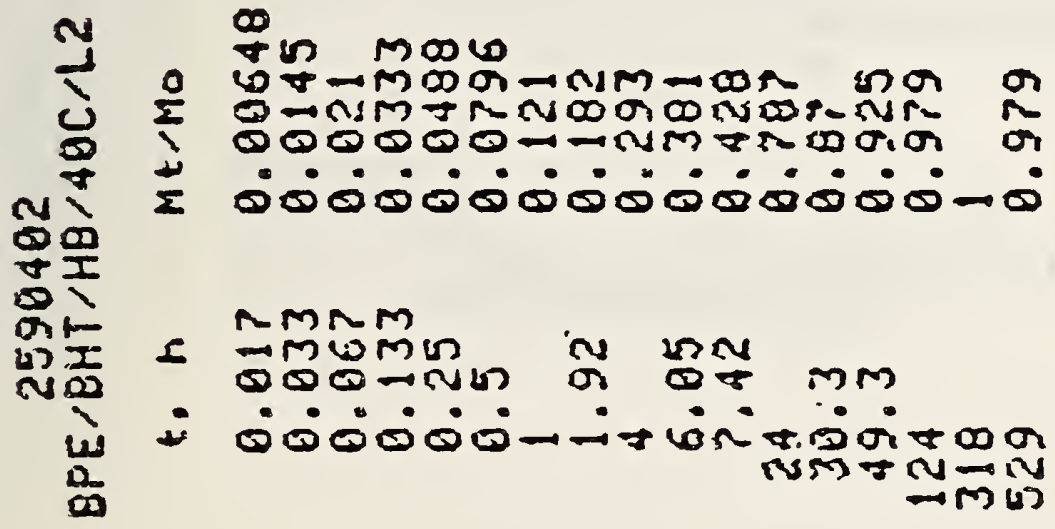

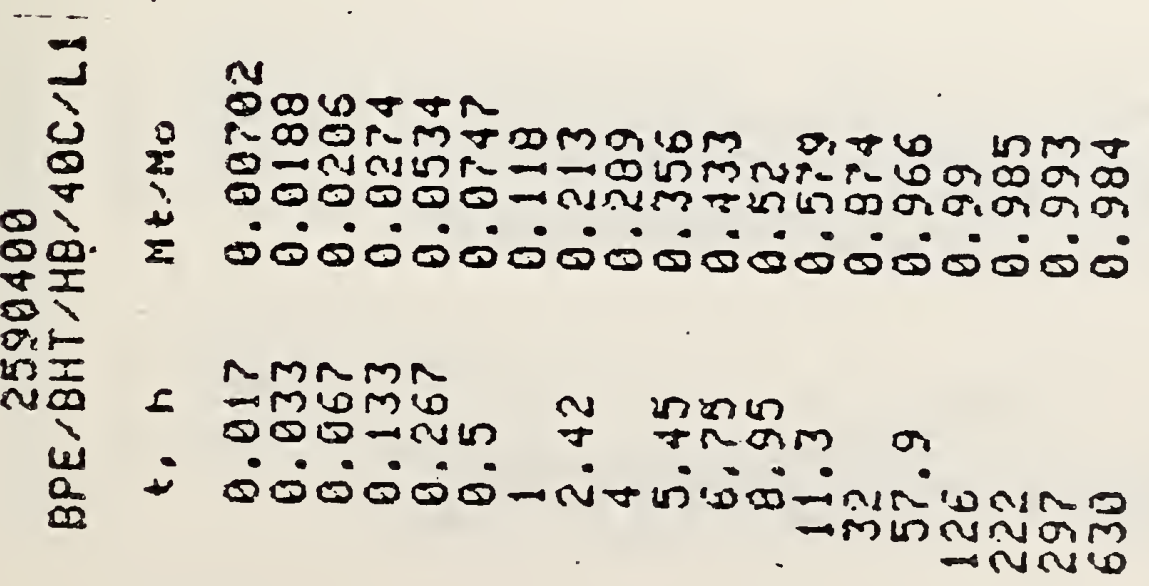



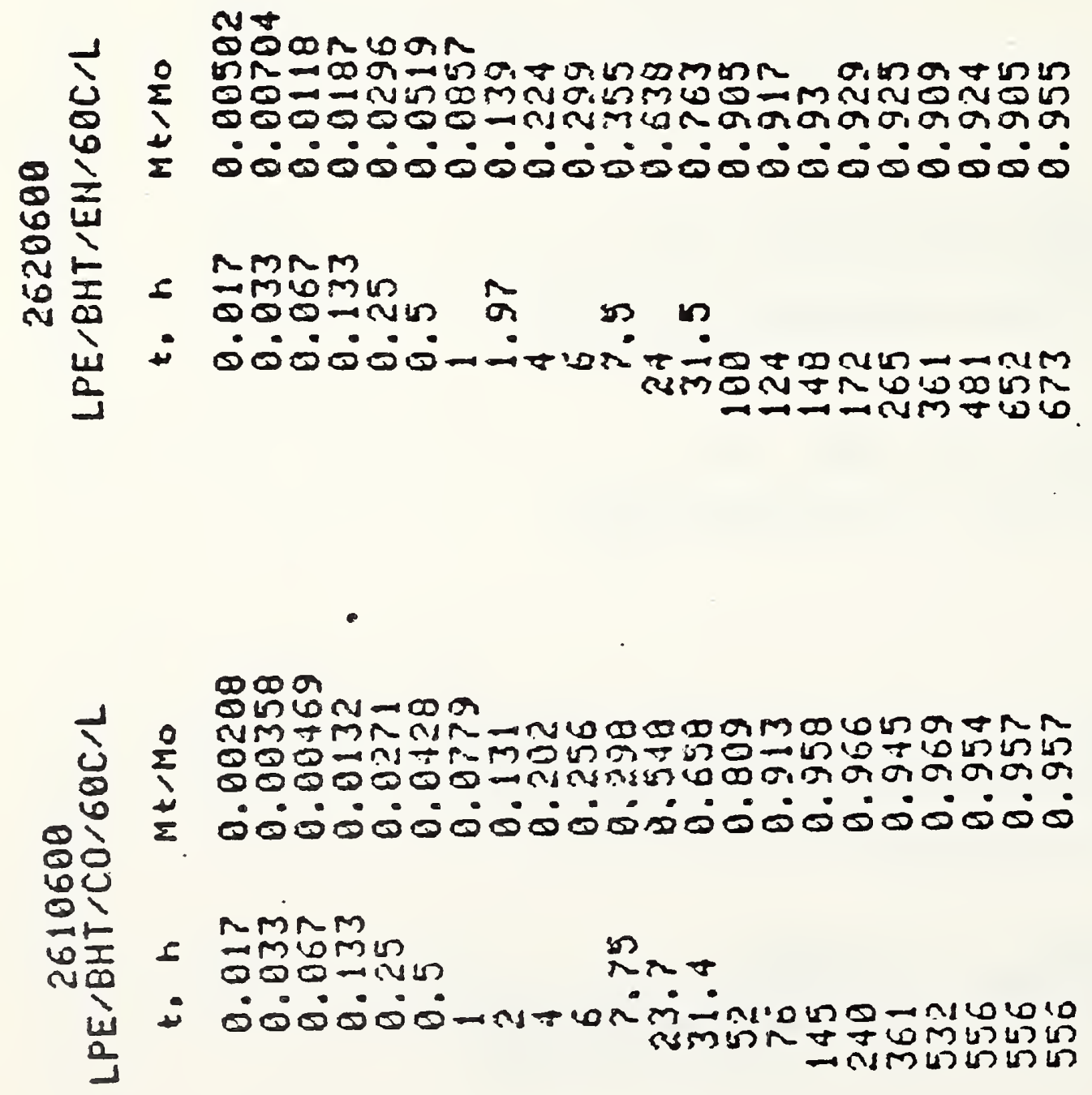


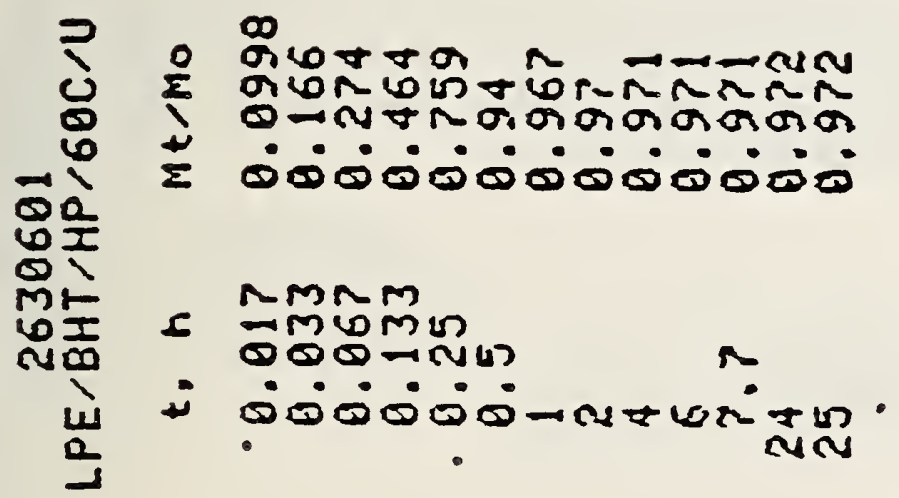

\footnotetext{
3 oñs

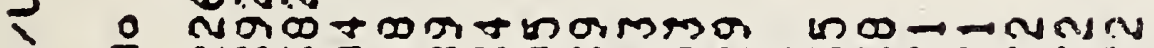

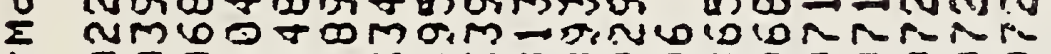

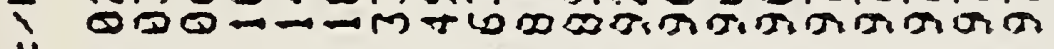

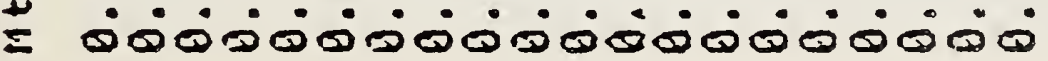

Q1

MI

ML

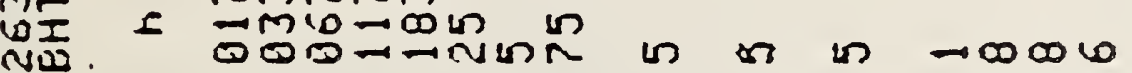

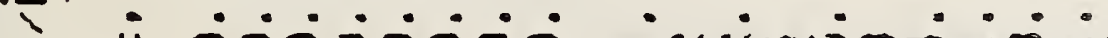
u +

a. 


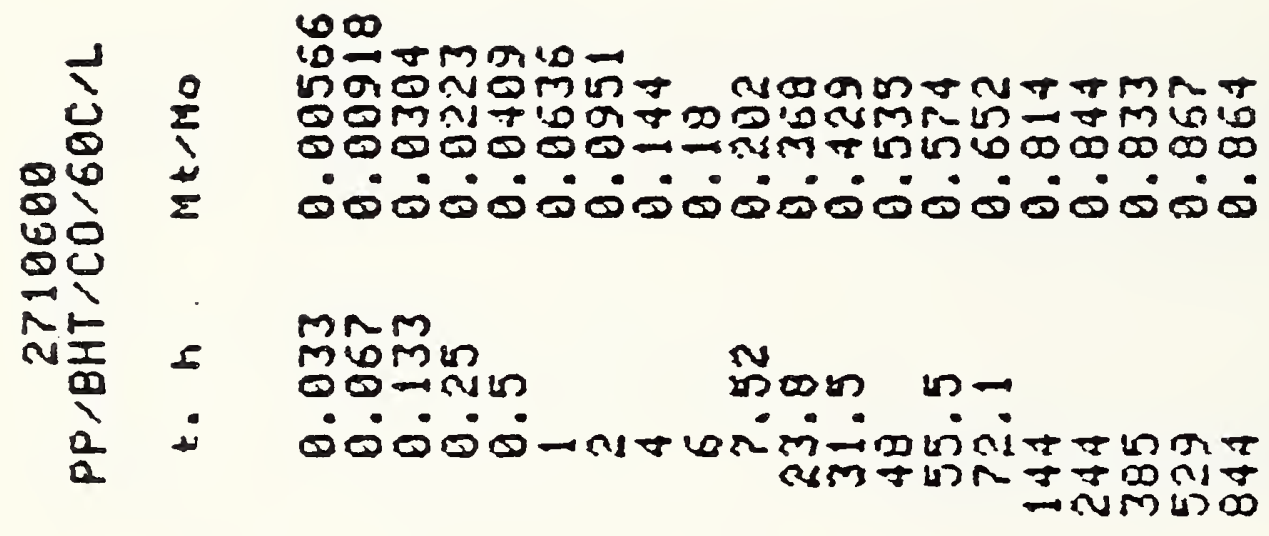

\footnotetext{
mon

omषuकष

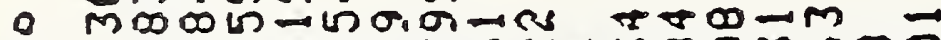

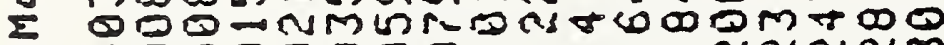

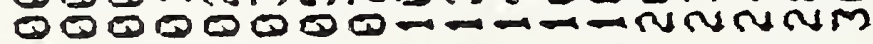

= ம்

$\infty$

$\infty 2$

m昌

$\infty$

NЕ

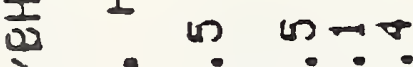

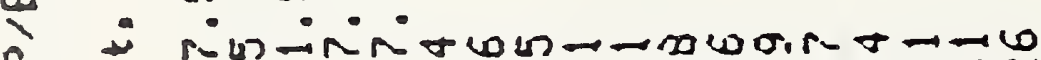

a nmitól.

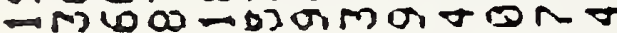
$\Rightarrow$ - N⿴囗十丁tu
} 


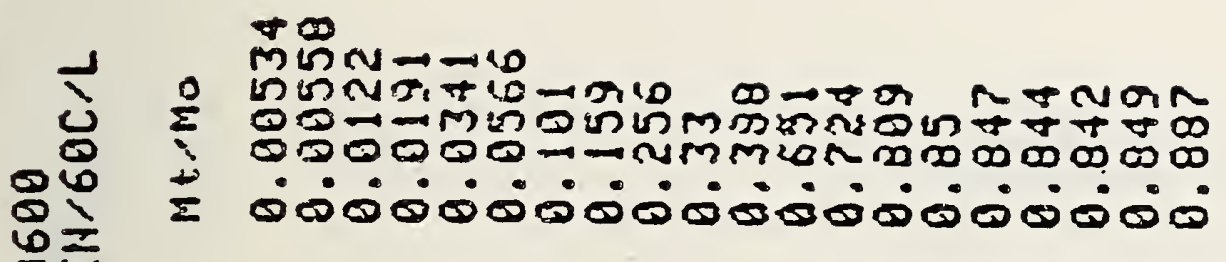

Dus

NE $\quad M m M$

NI 5 =M⿻日禸

D $0000 n$ in

i

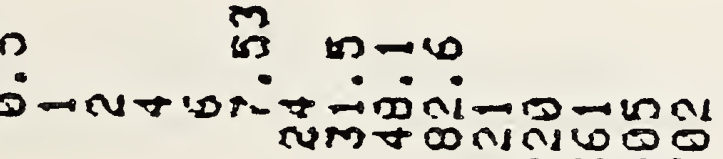

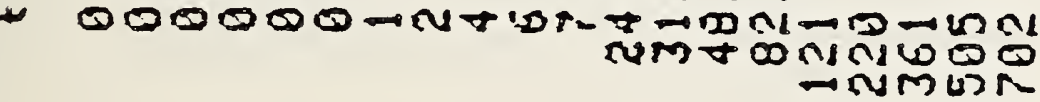

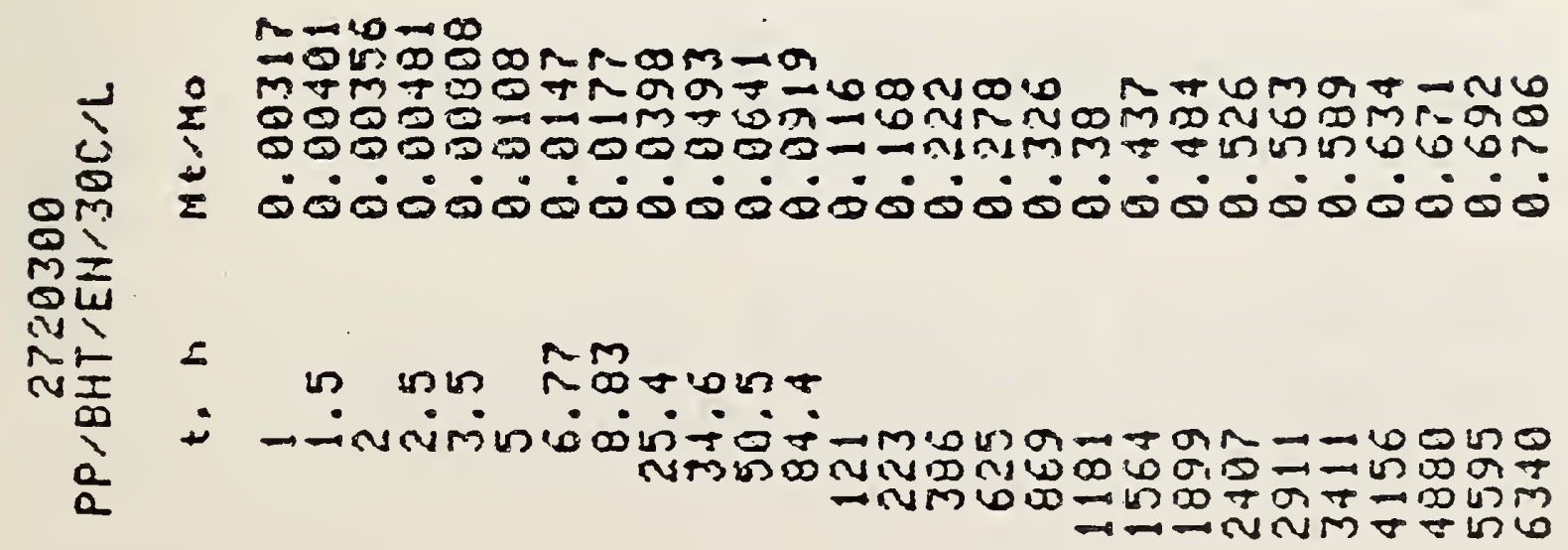

$\frac{2}{2}$ 

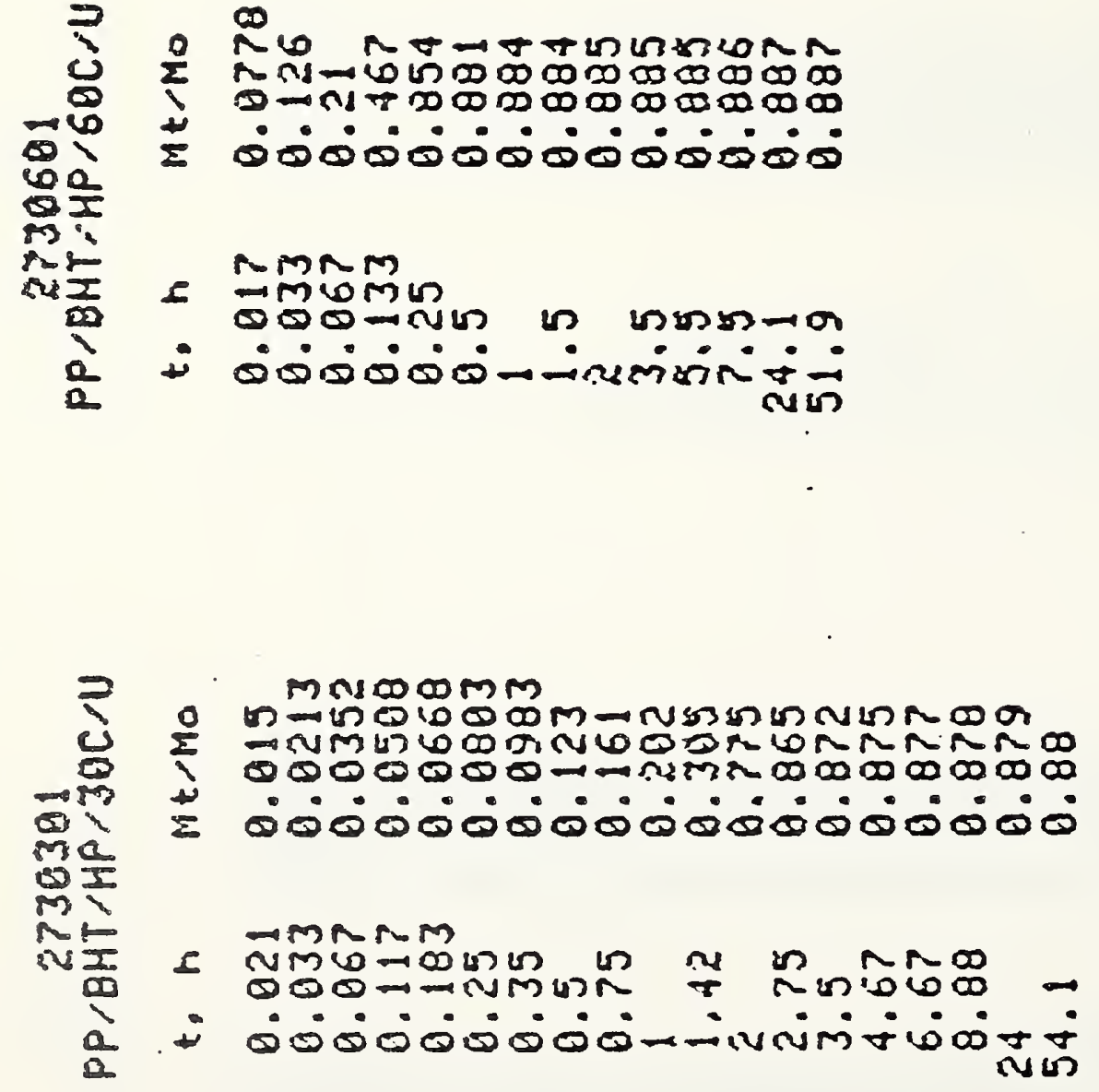


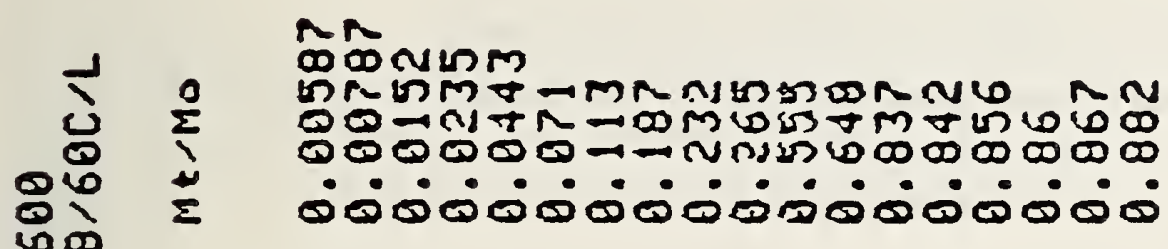

$m \cap m$

momin un

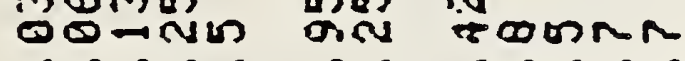

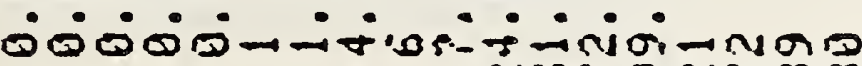

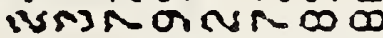

$\rightarrow \infty$ is

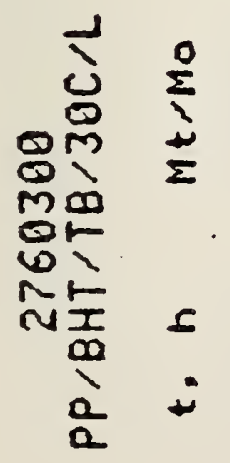

$+\infty \cos 0$

$1-\sigma 0 R-3000 \%$

U-Mm

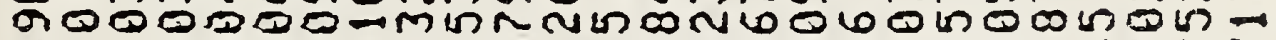

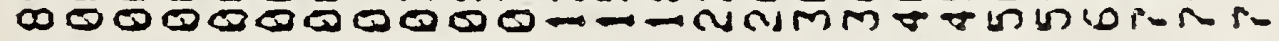
గம் ம் ம் (

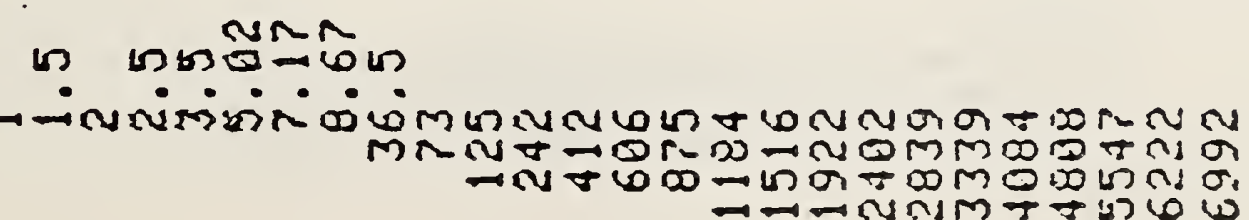




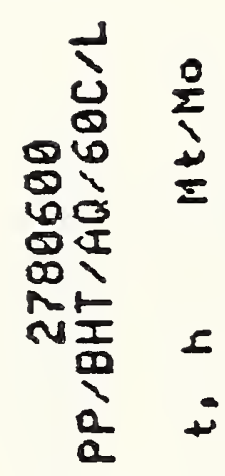

\section{$\forall N$}

nーARnM $\infty$

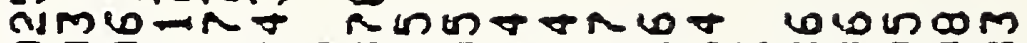

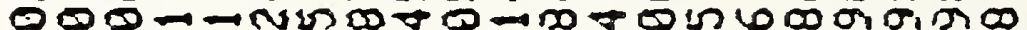

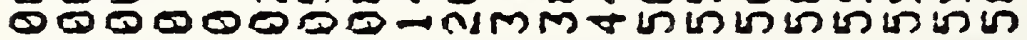

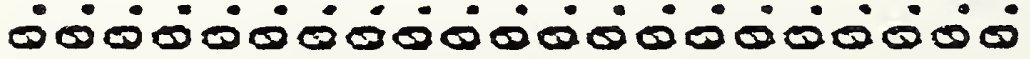

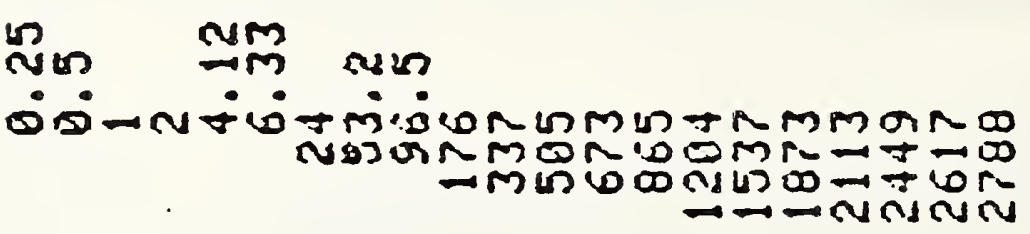

$+a$

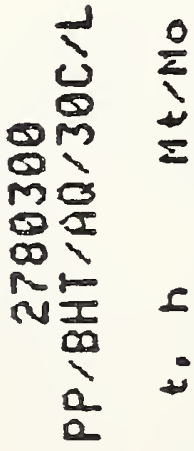




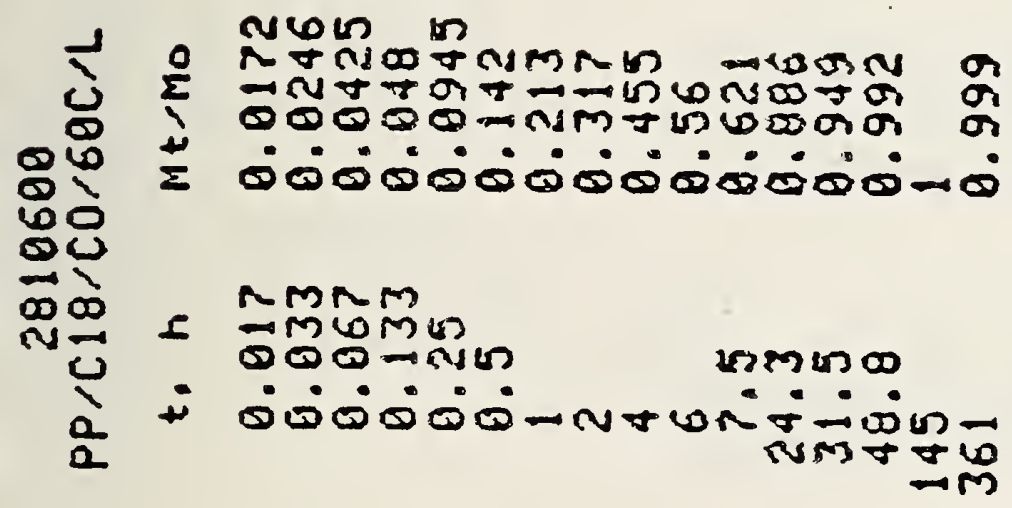

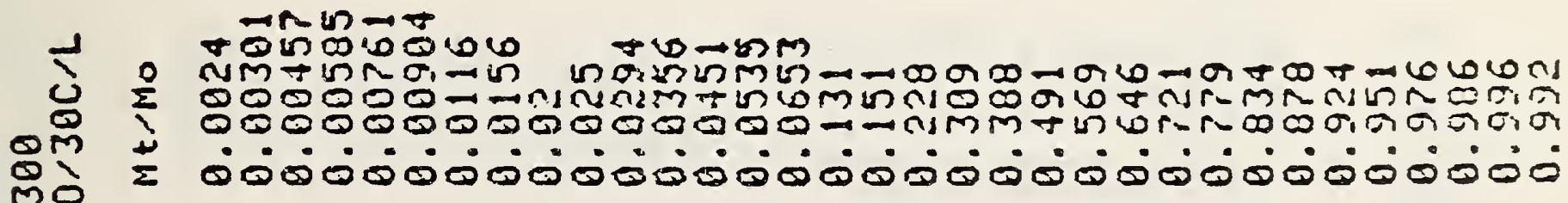
ou $\rightarrow 1$

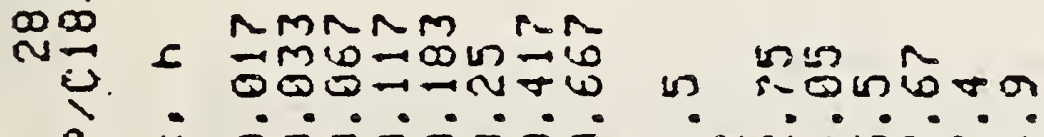

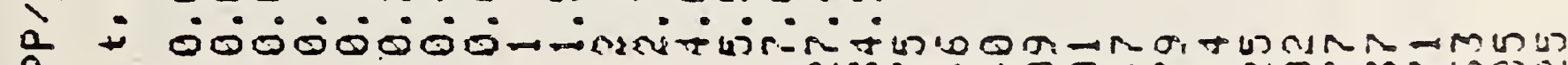
NMR VDOUR-RIDRMN

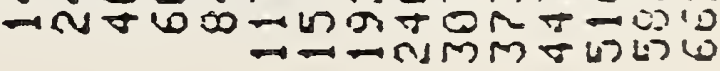



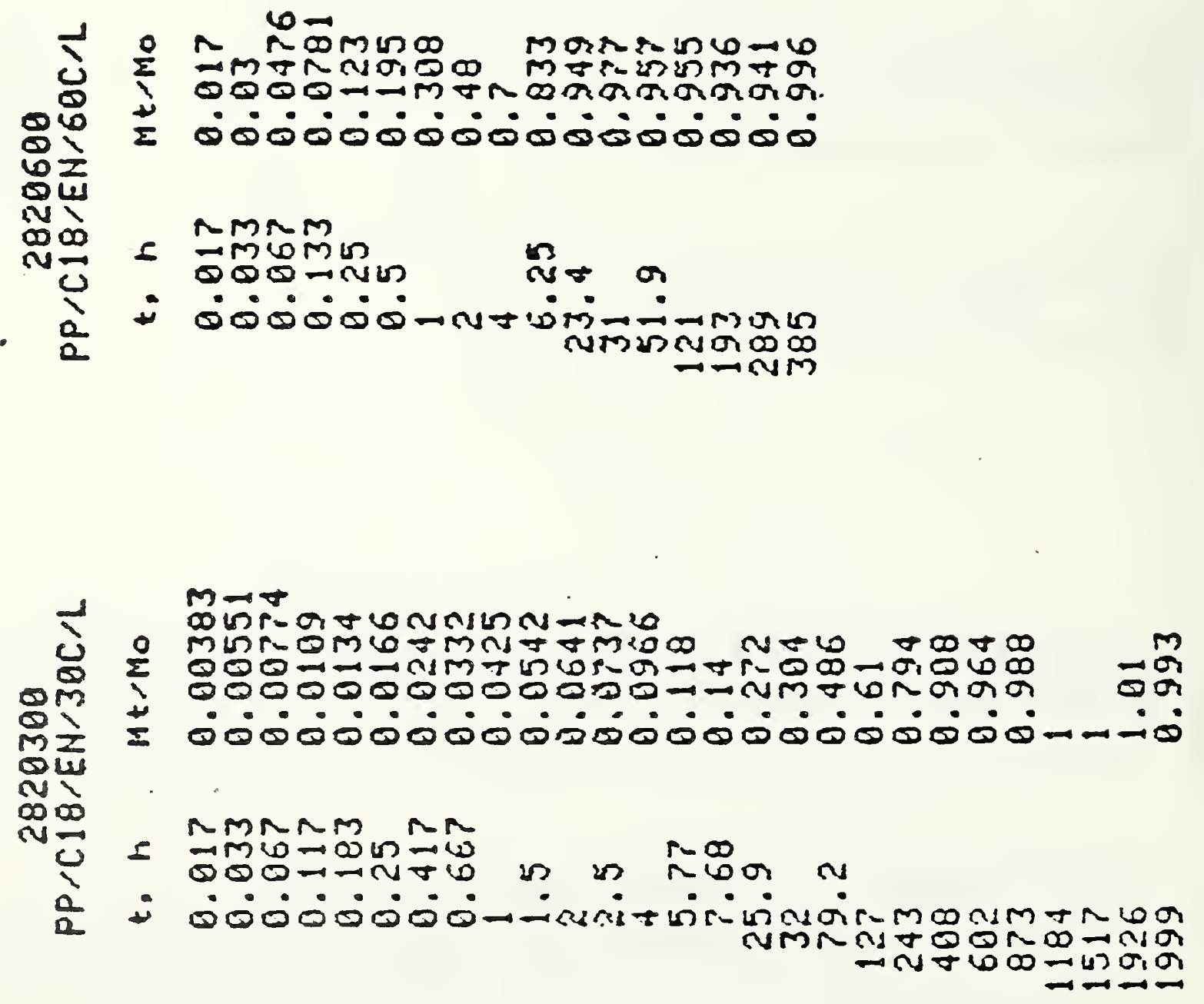


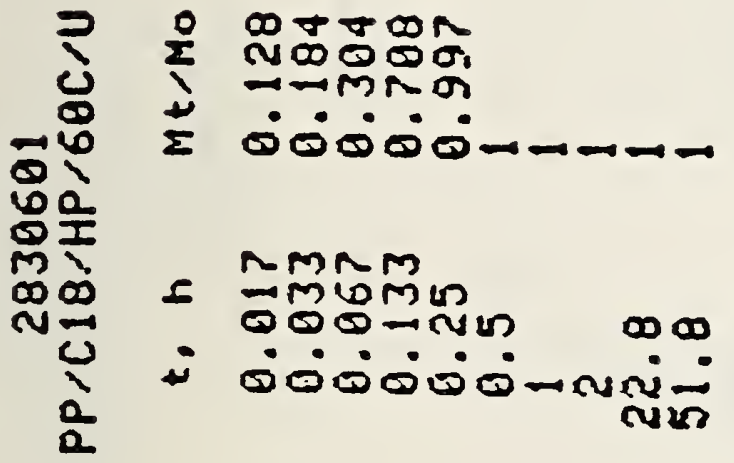

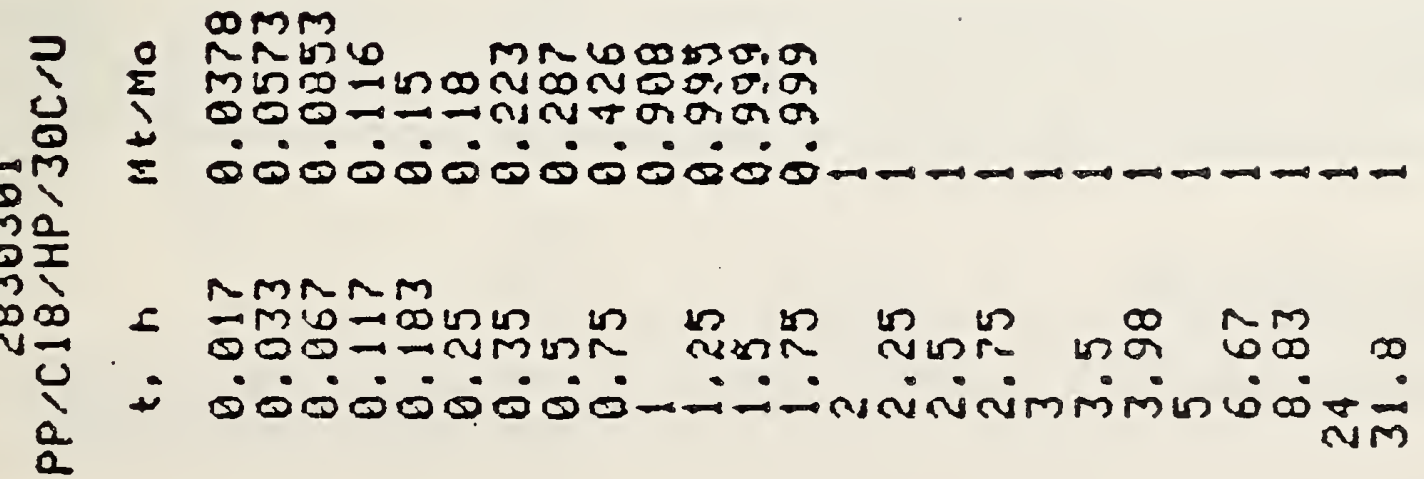



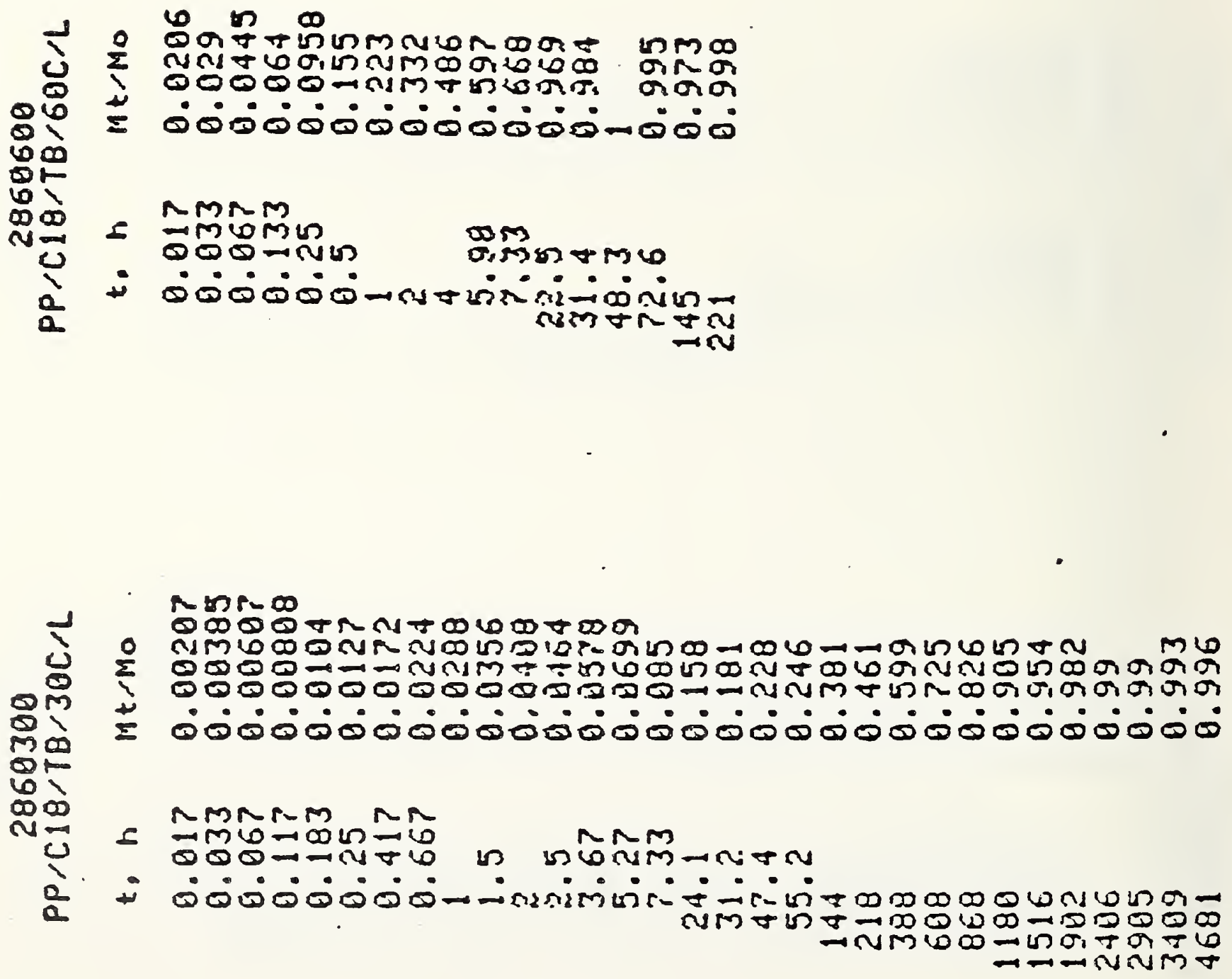


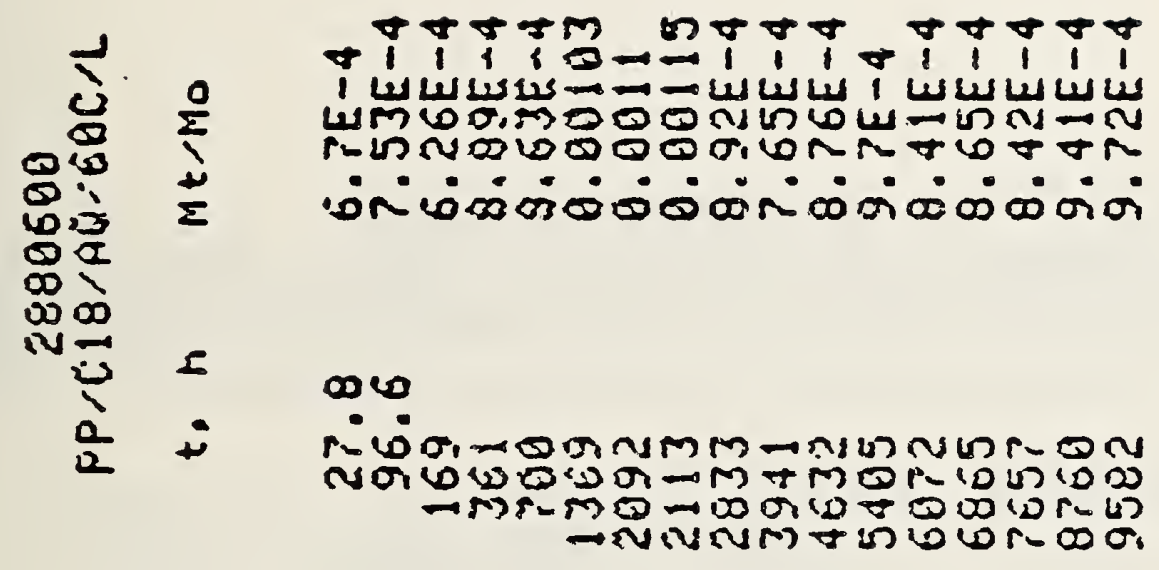

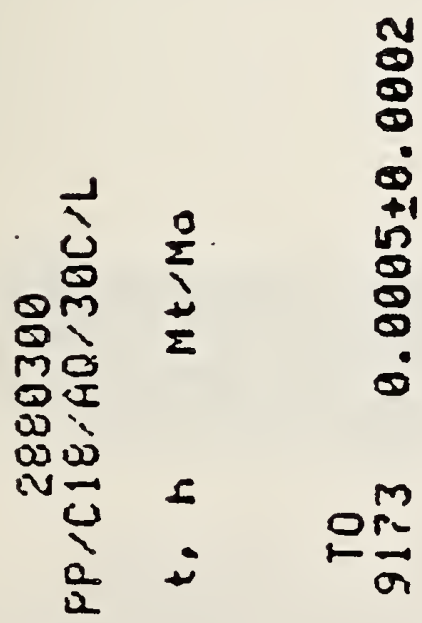




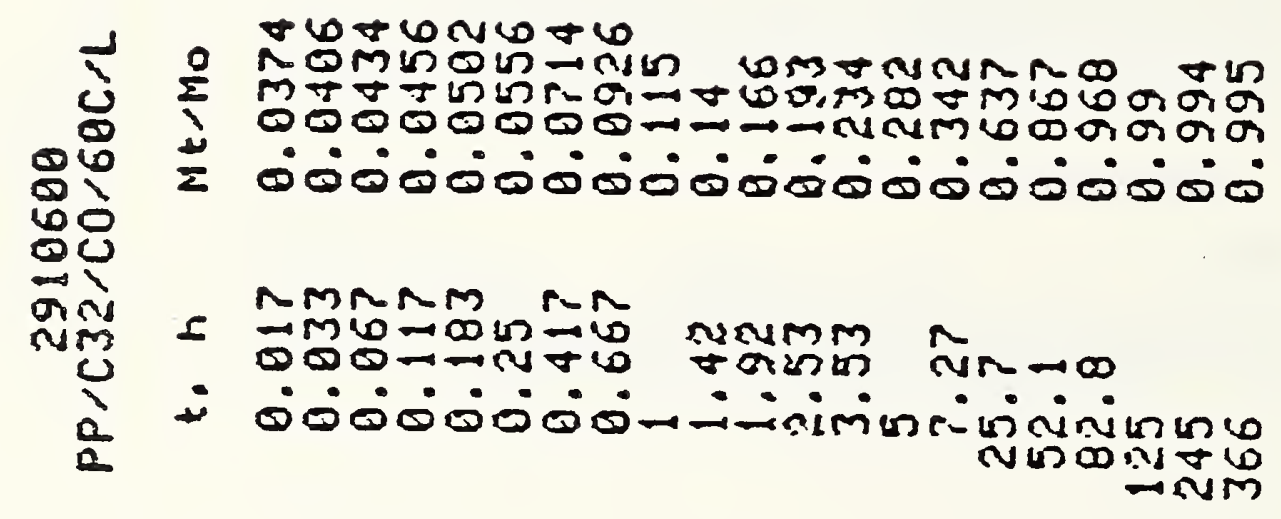

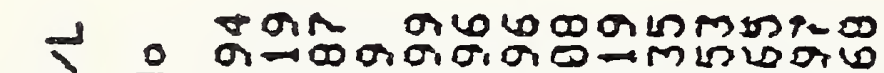

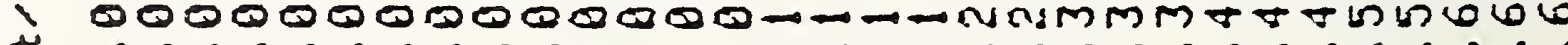

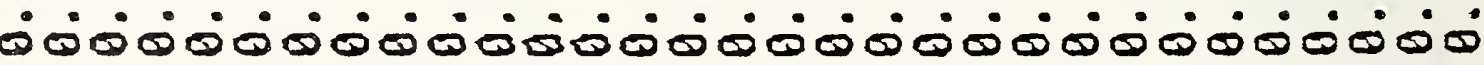

MO

$\infty$

बin

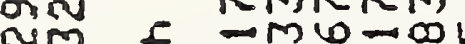

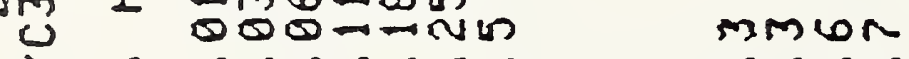

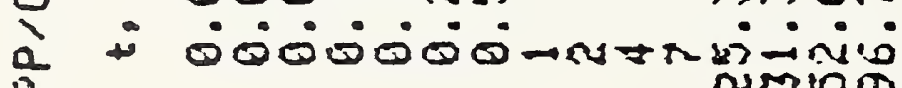

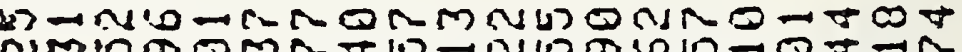

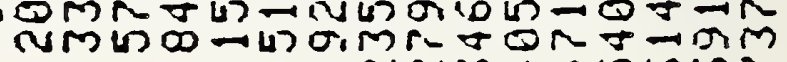




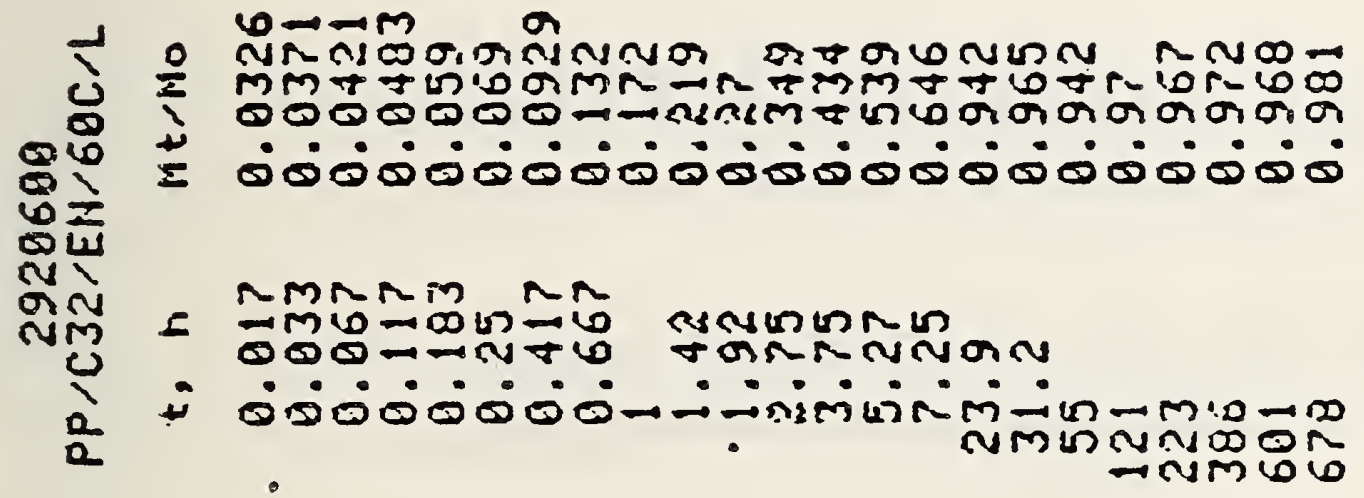

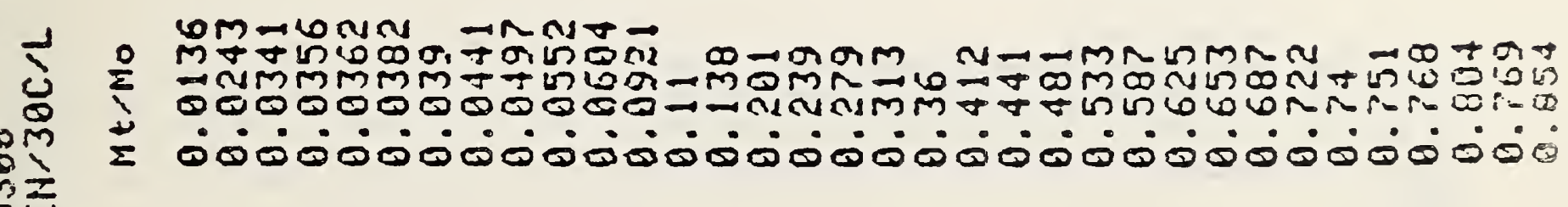

MMNM

- $\rightarrow$ minto

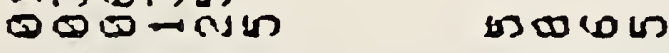

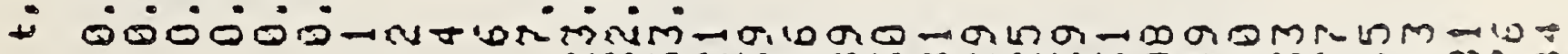

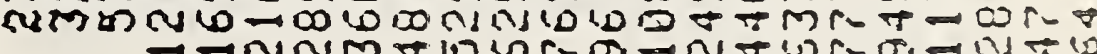

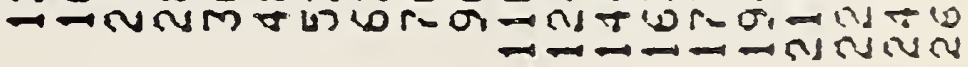



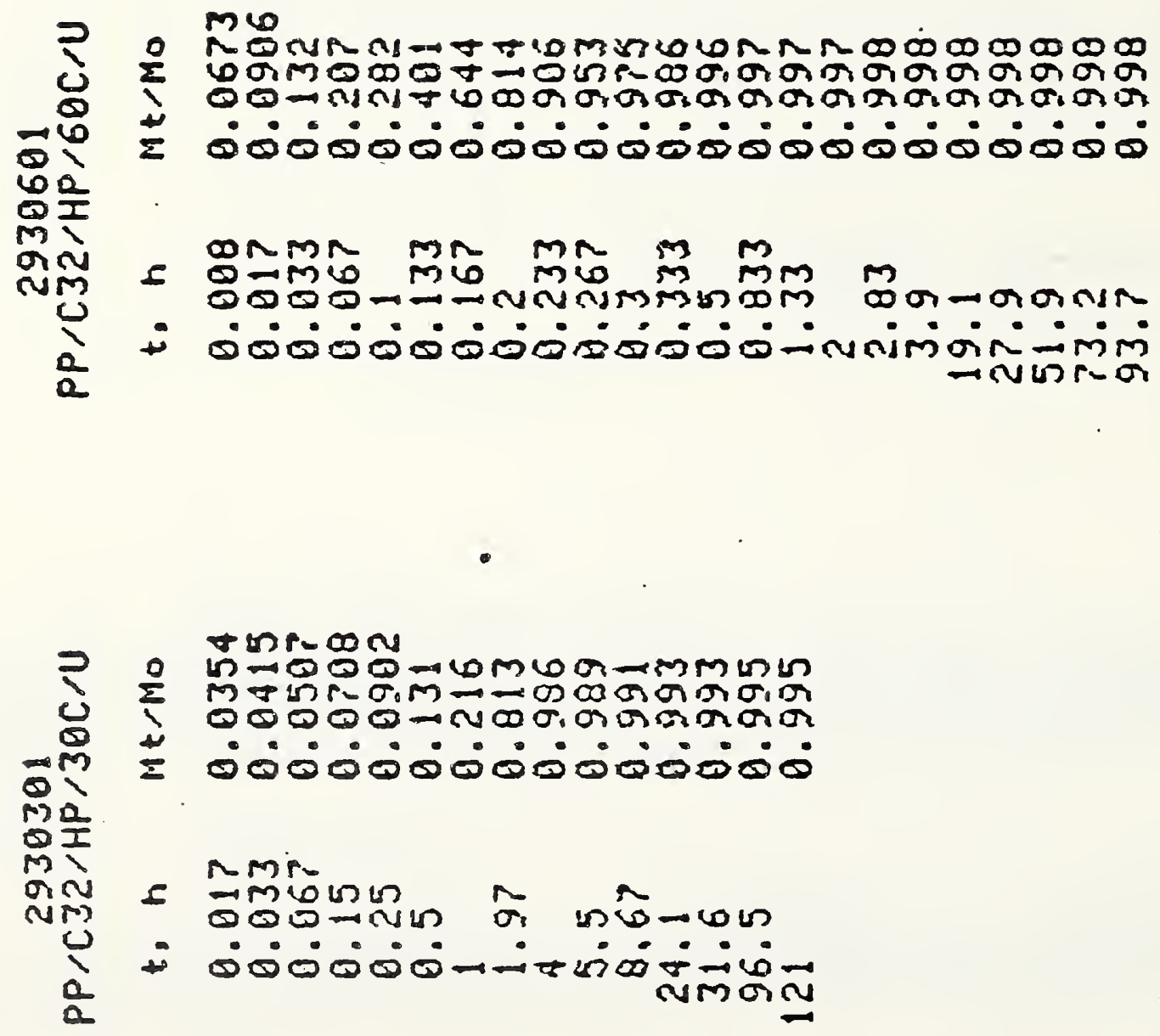


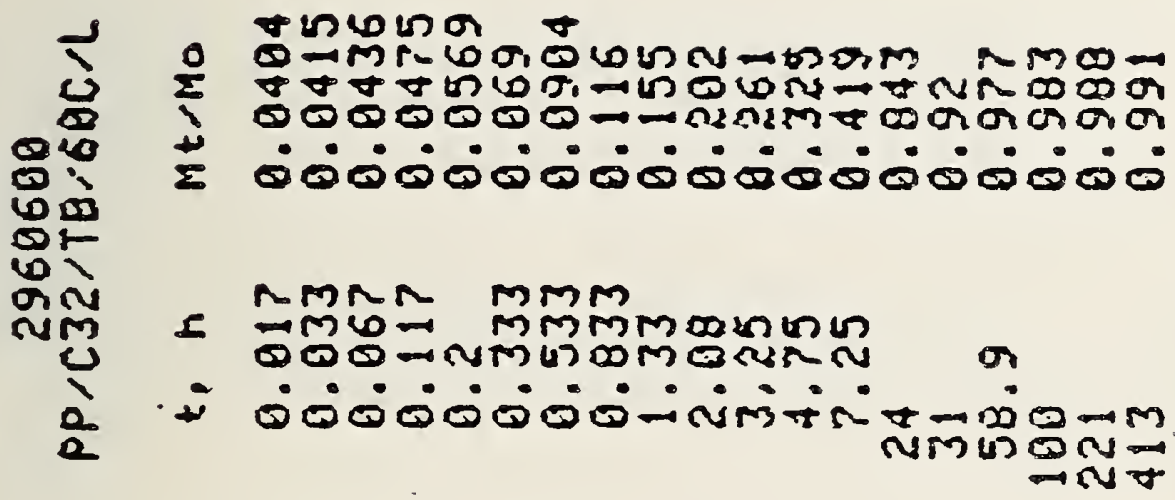

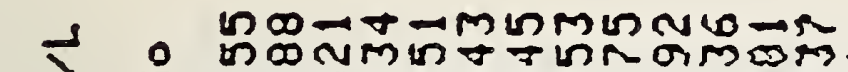

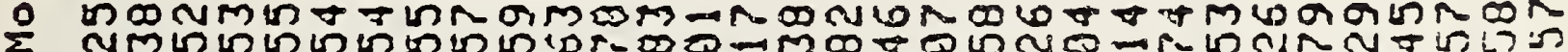

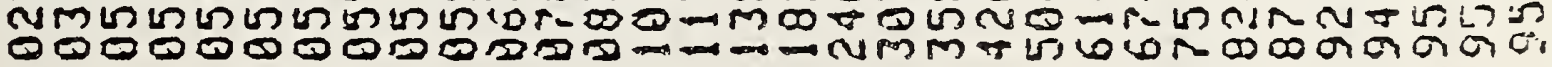
Е ن 0 anc ivim

L $\rightarrow m 6=\infty$

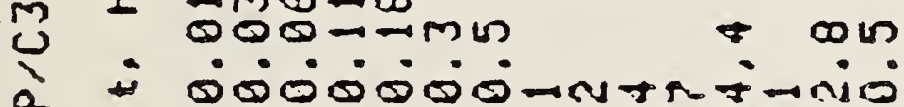

2 NMind - CUM OM - $=$ as Cum
} 


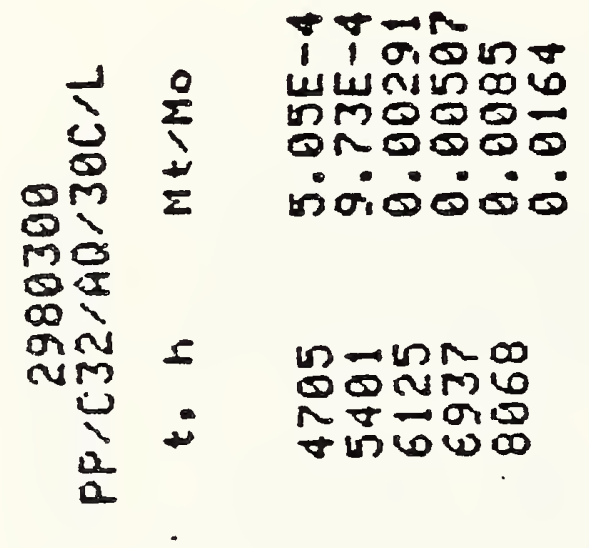

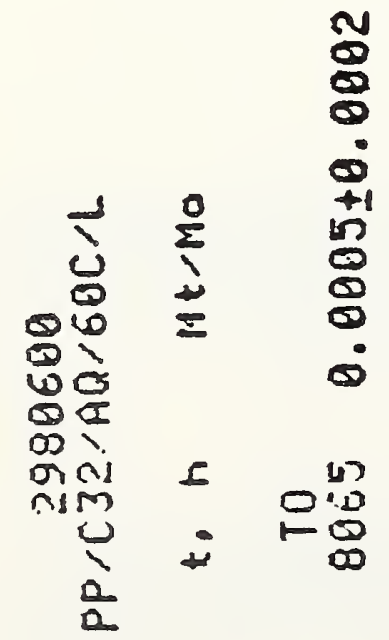




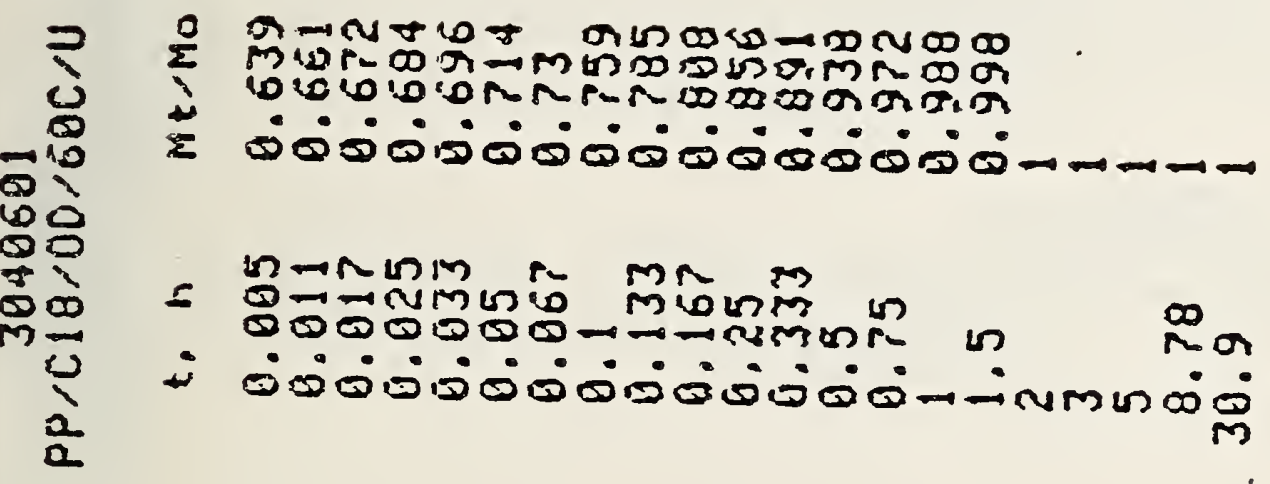

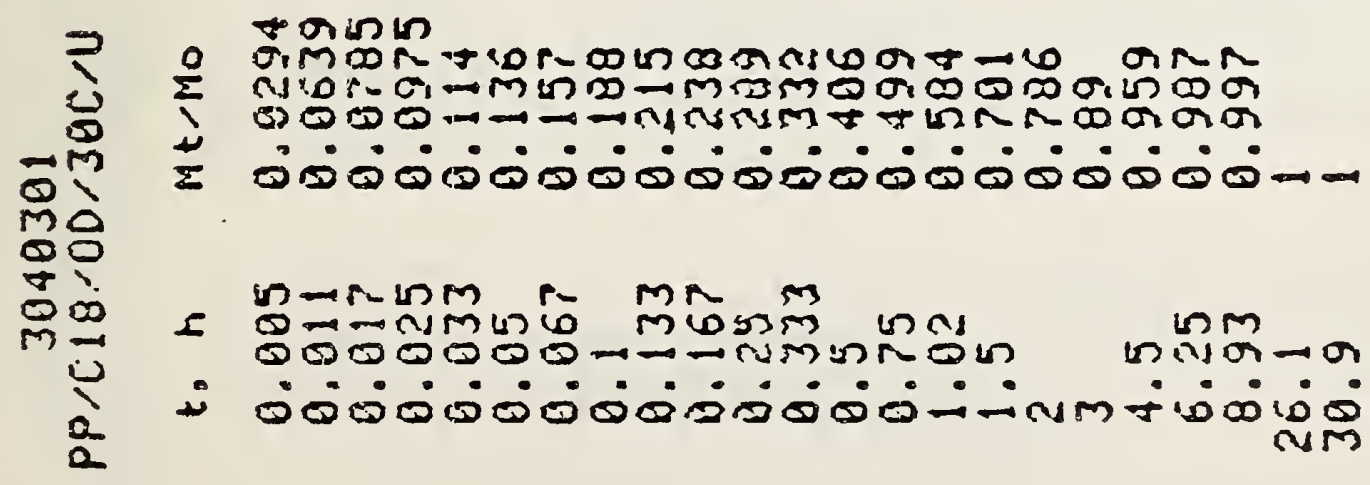



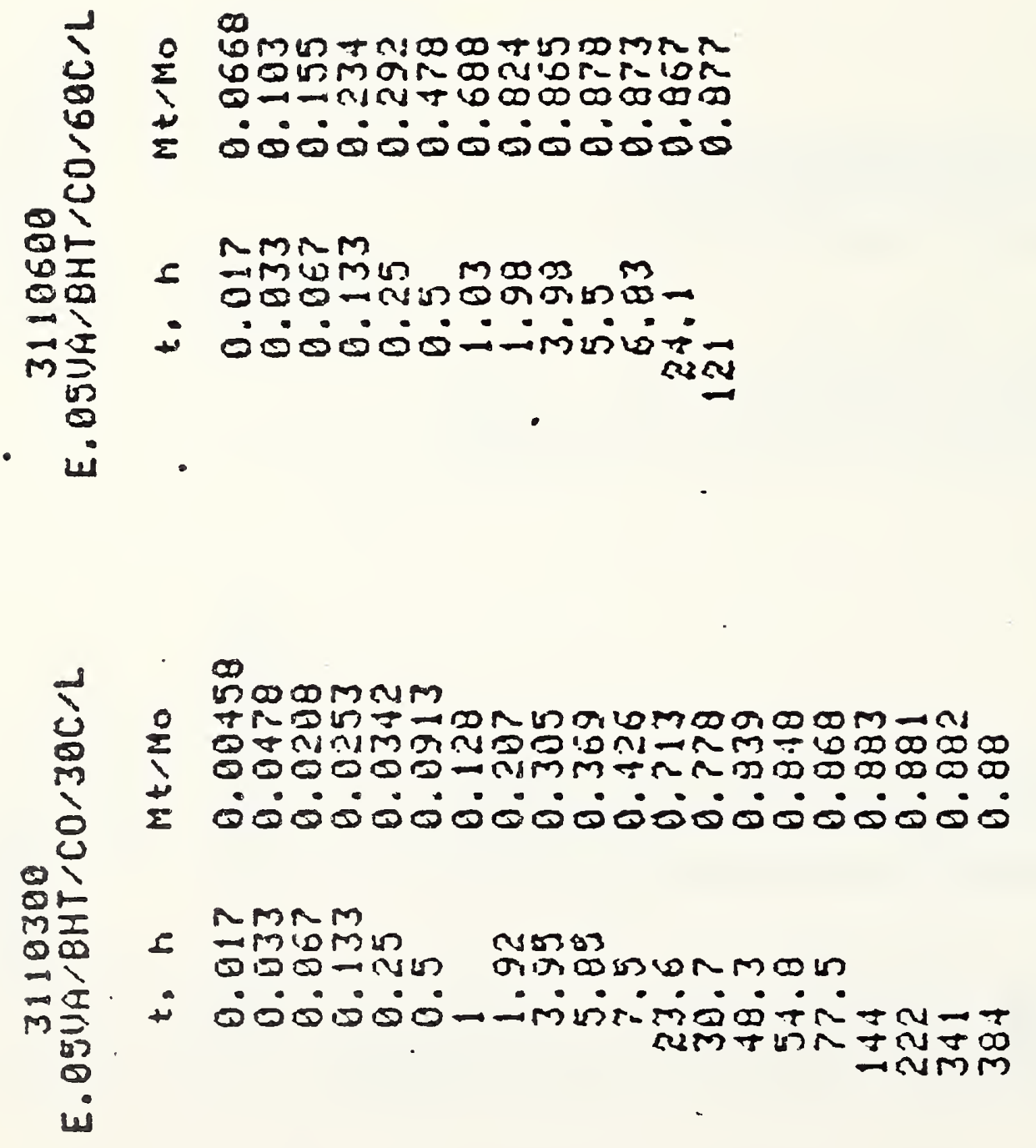

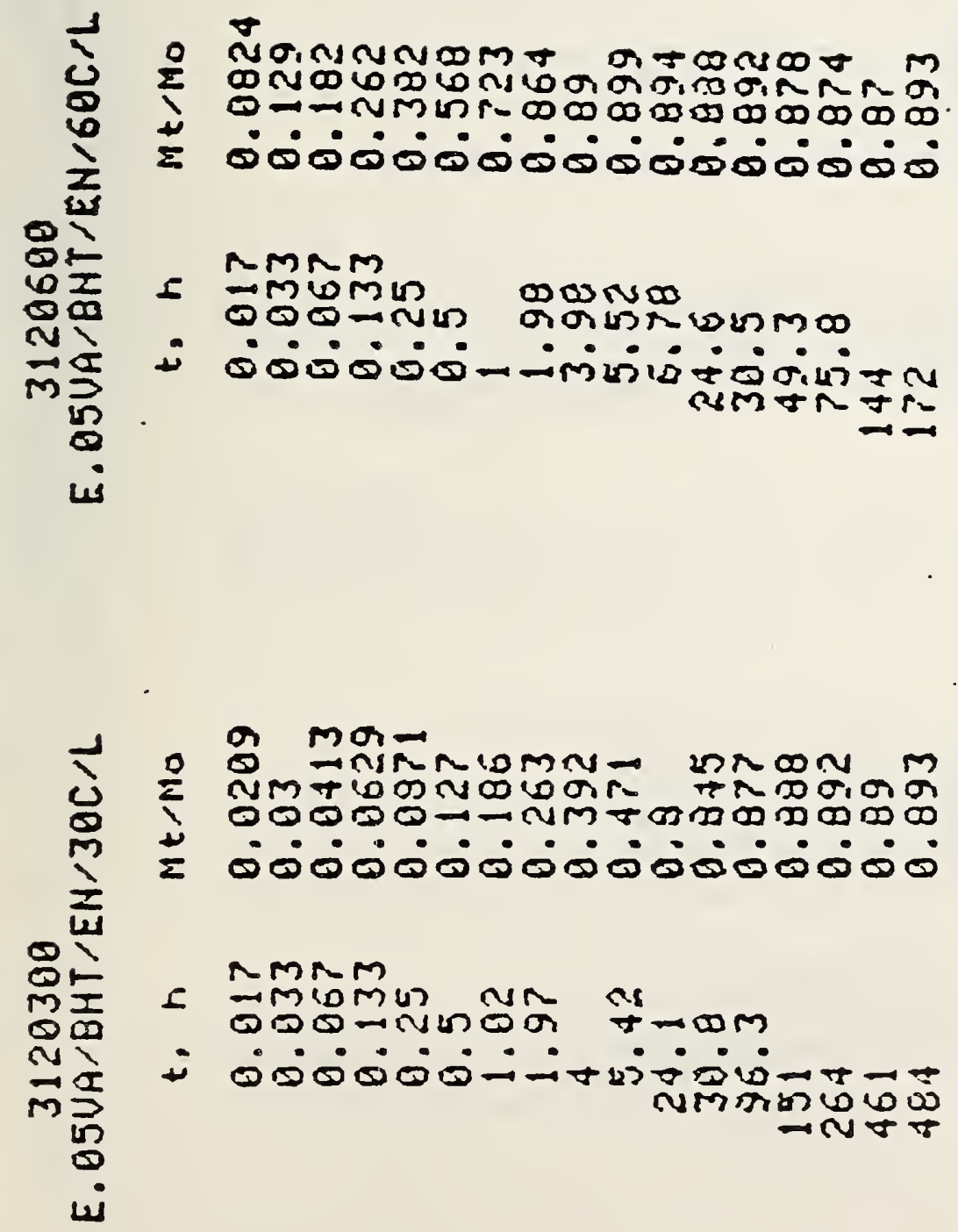


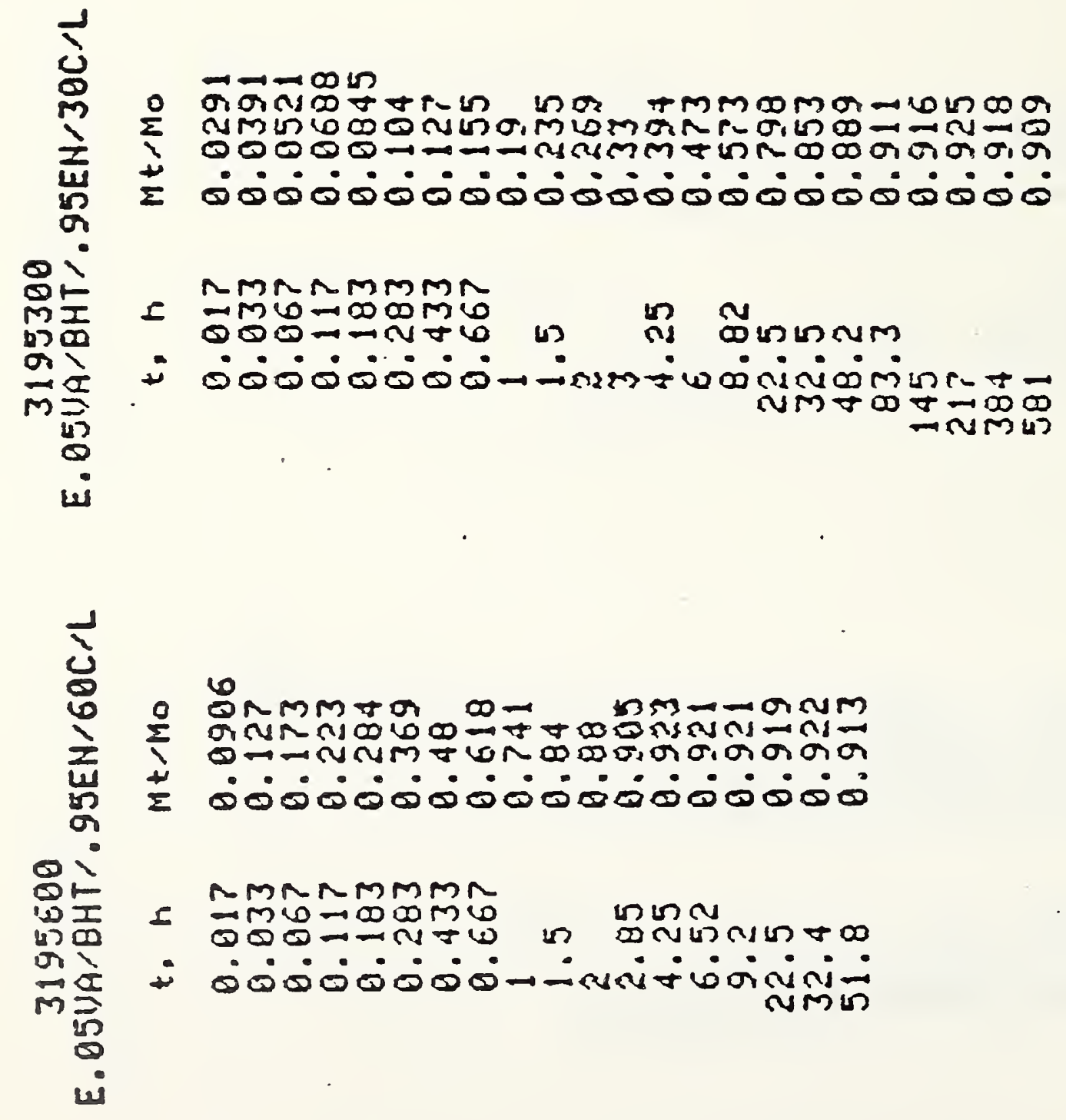




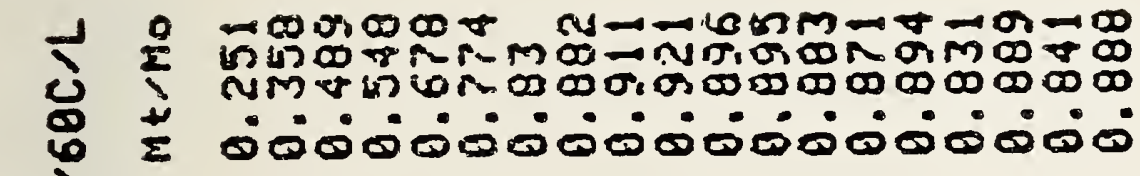

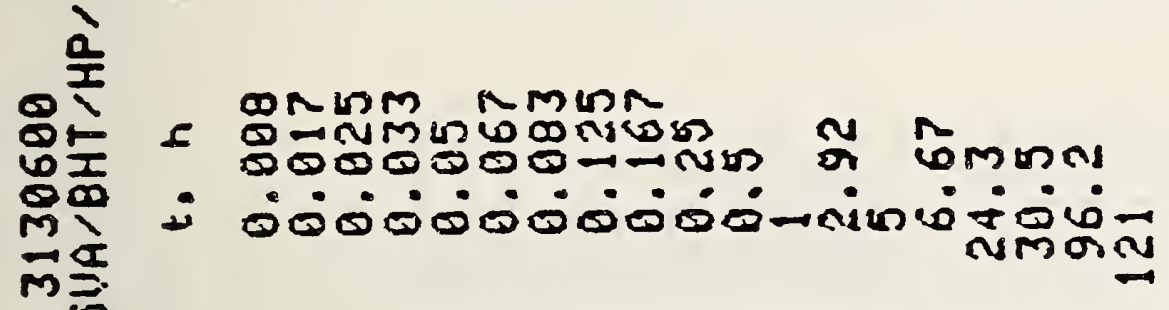

M

。

u

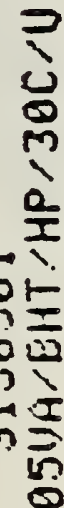

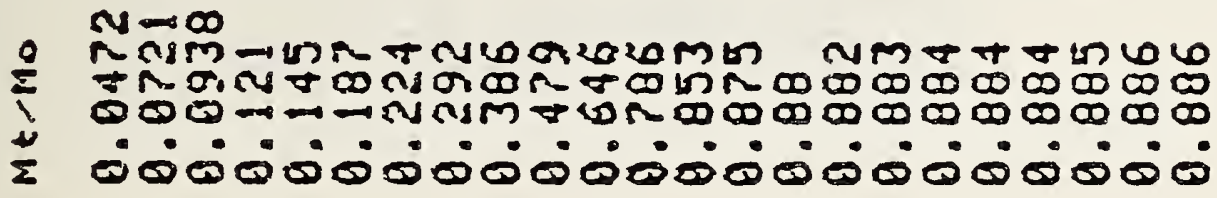

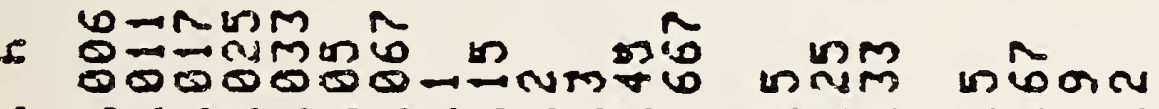
- ن in

نं

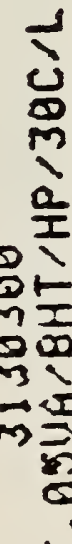

- n-Nonmutrummmat

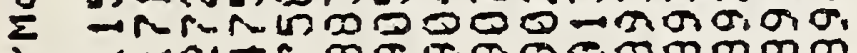

- i் க்

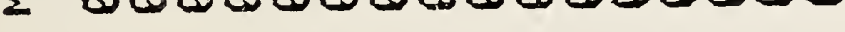
MMRm
I $\rightarrow$ momu
$000-(4)$
6 $m M$
Q DMMN-
+
तथ $\rightarrow 0$ 

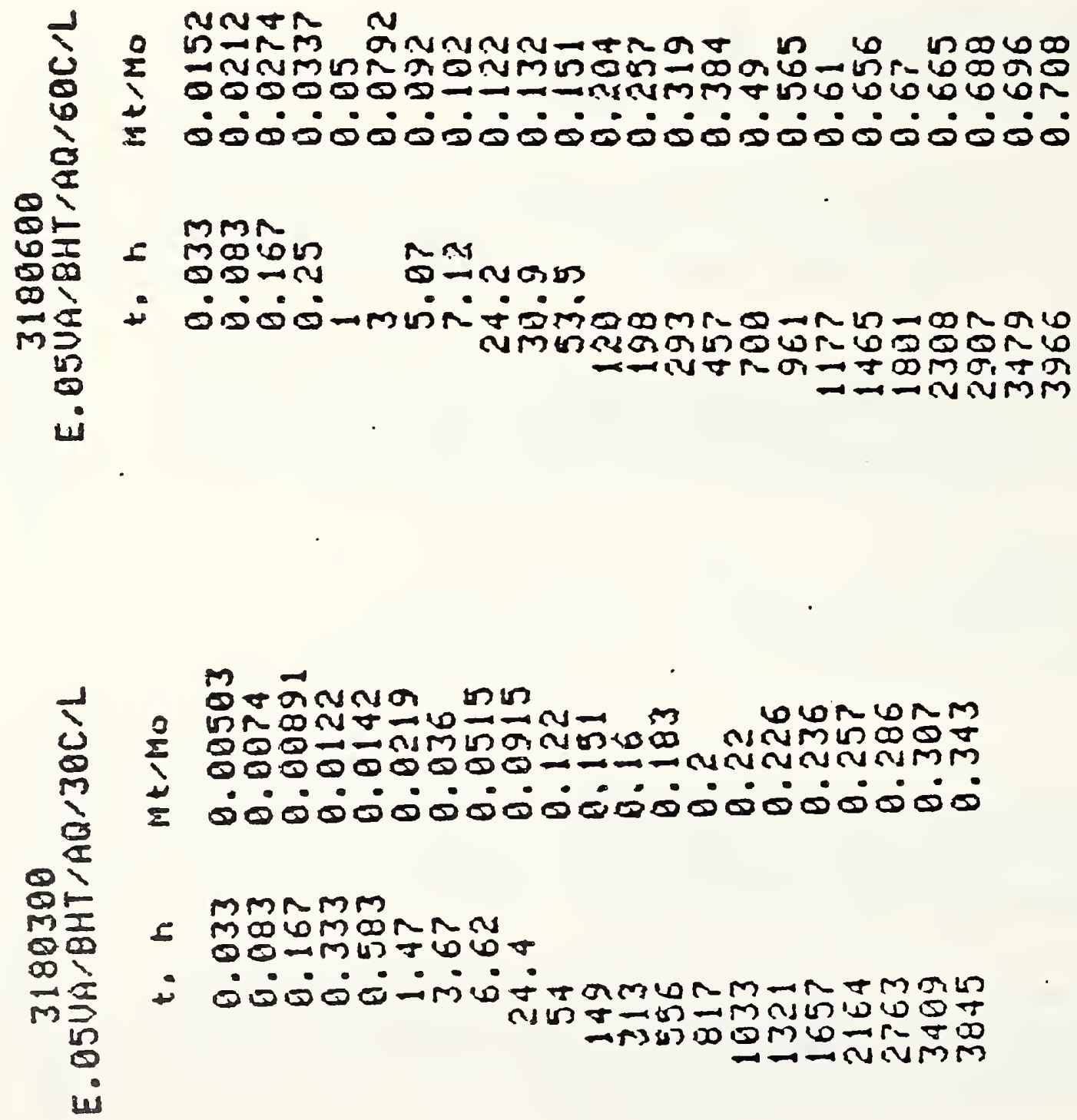


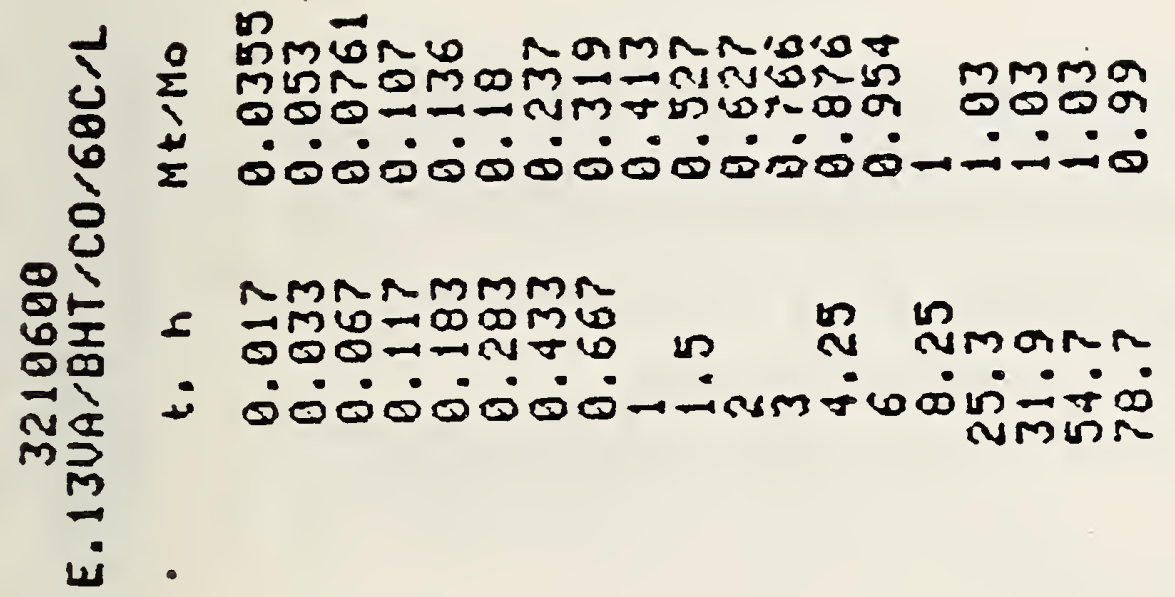

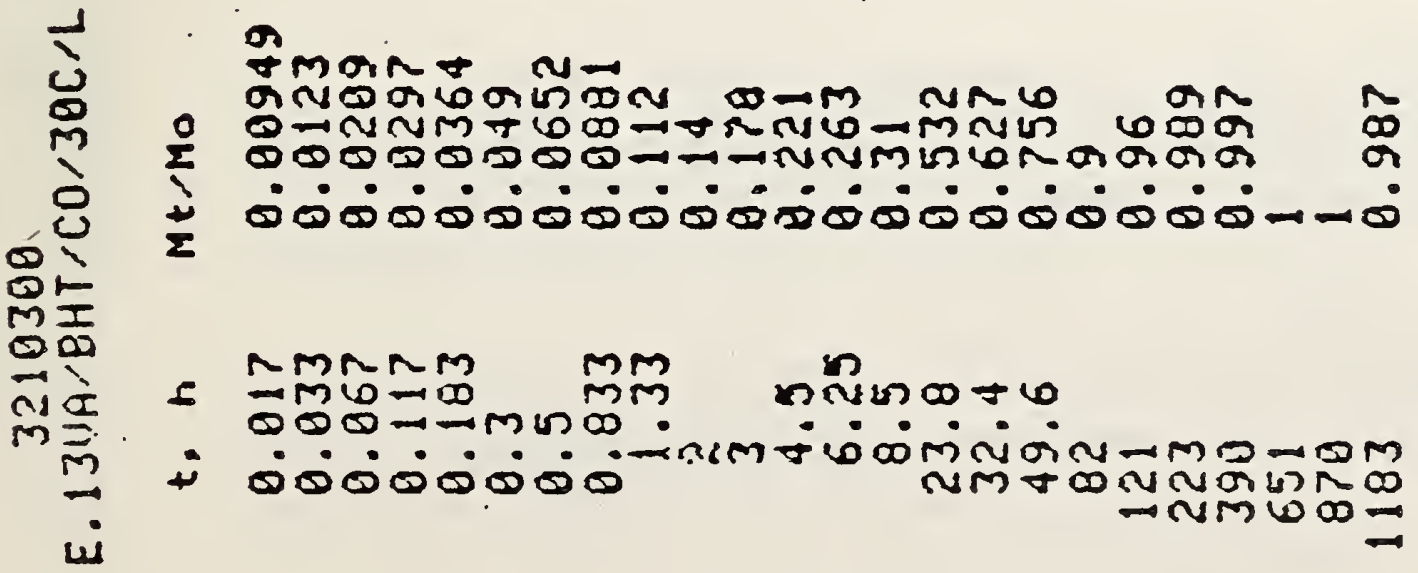




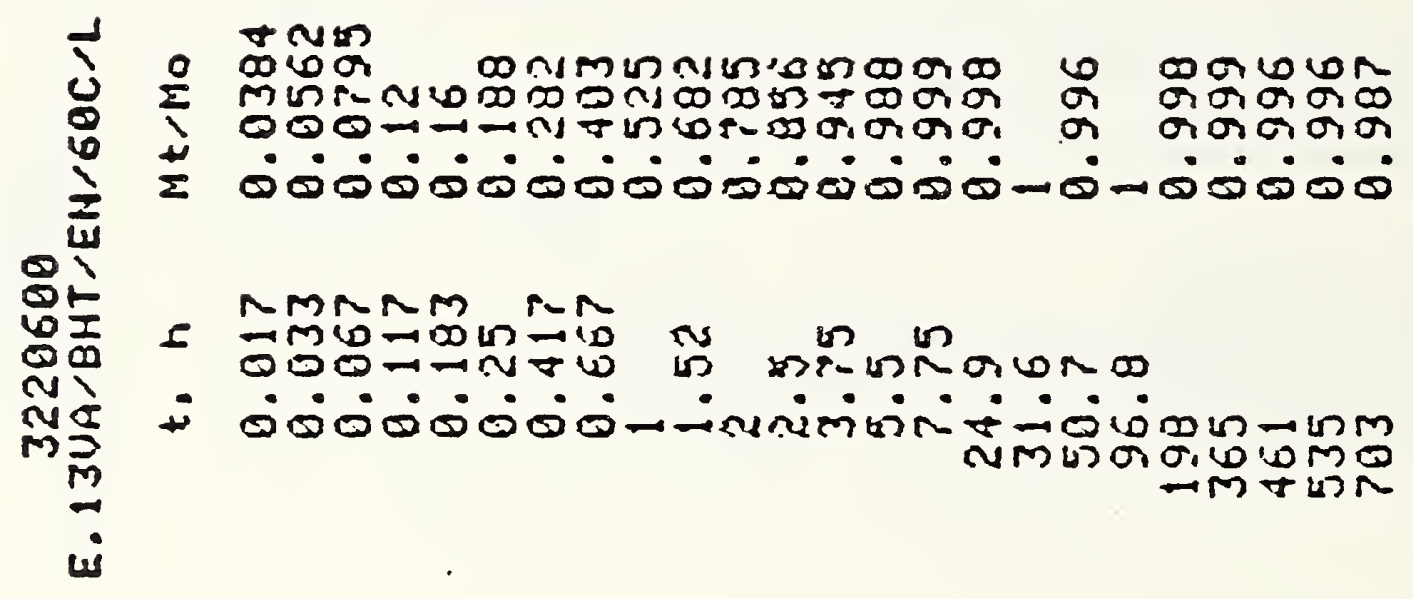

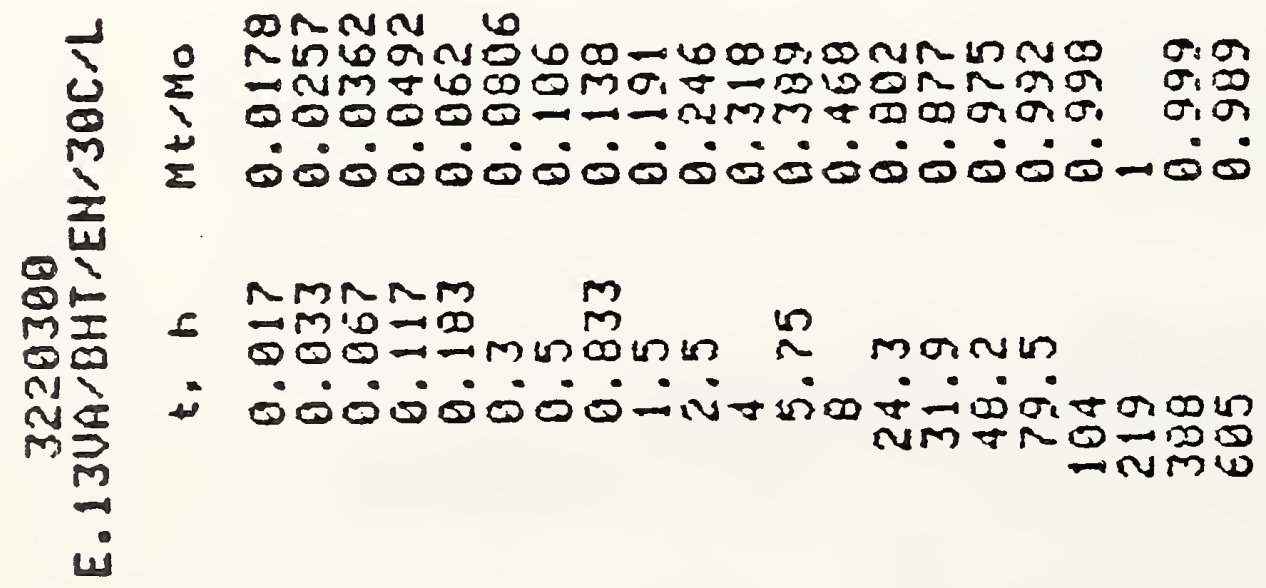




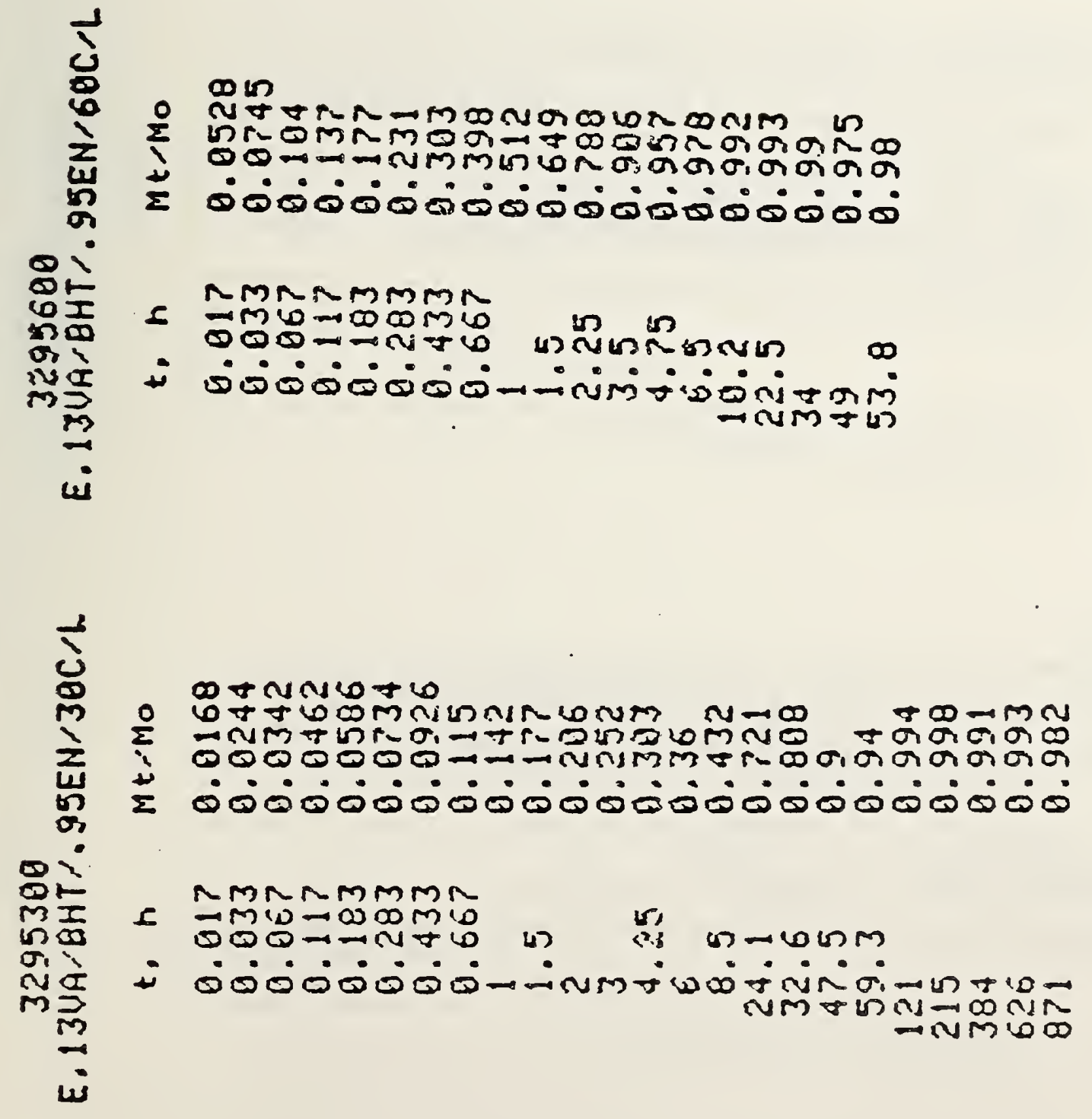




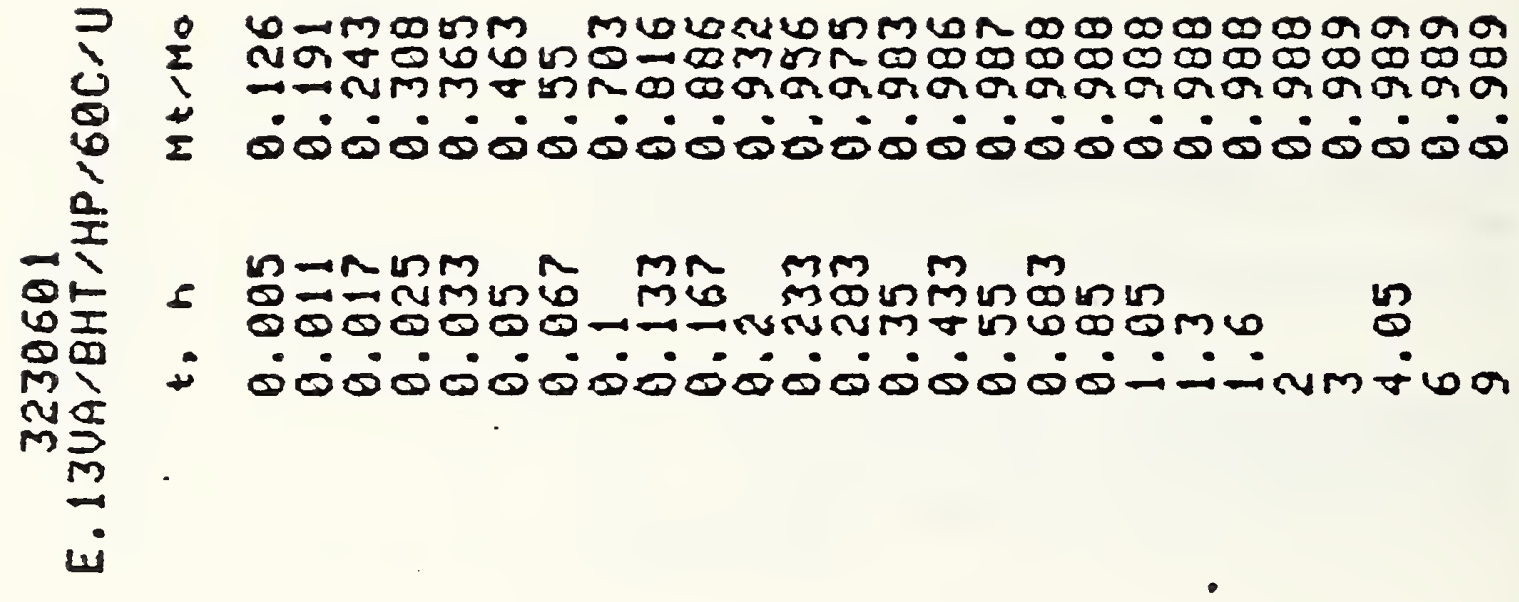

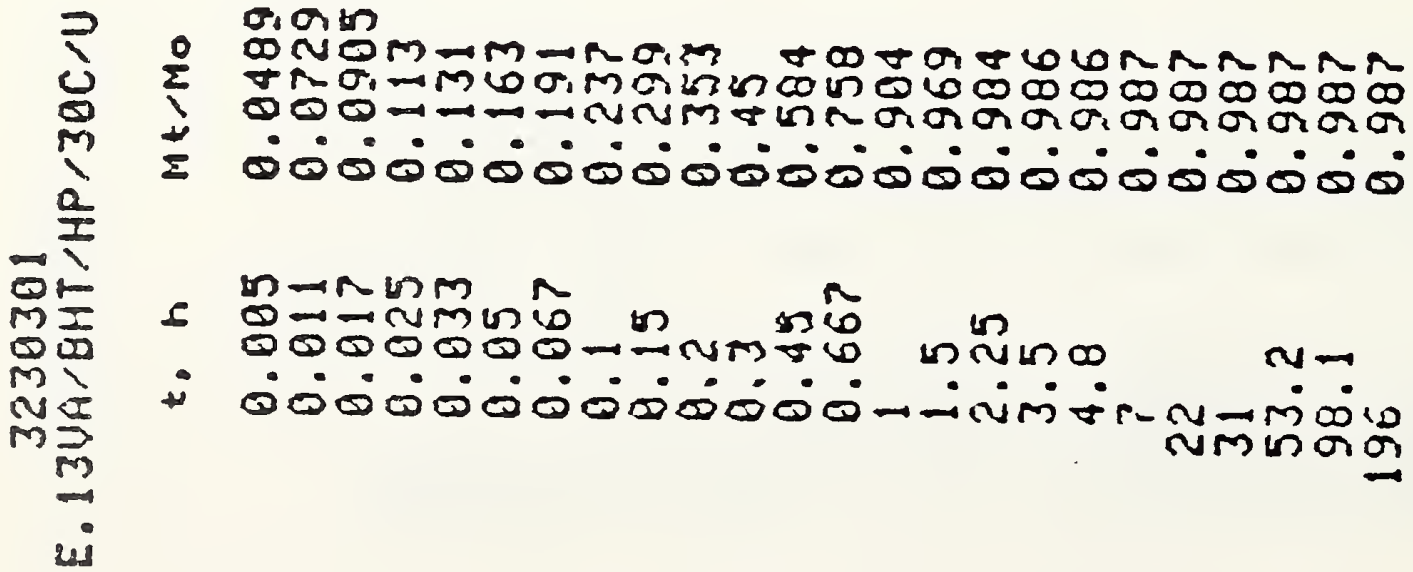




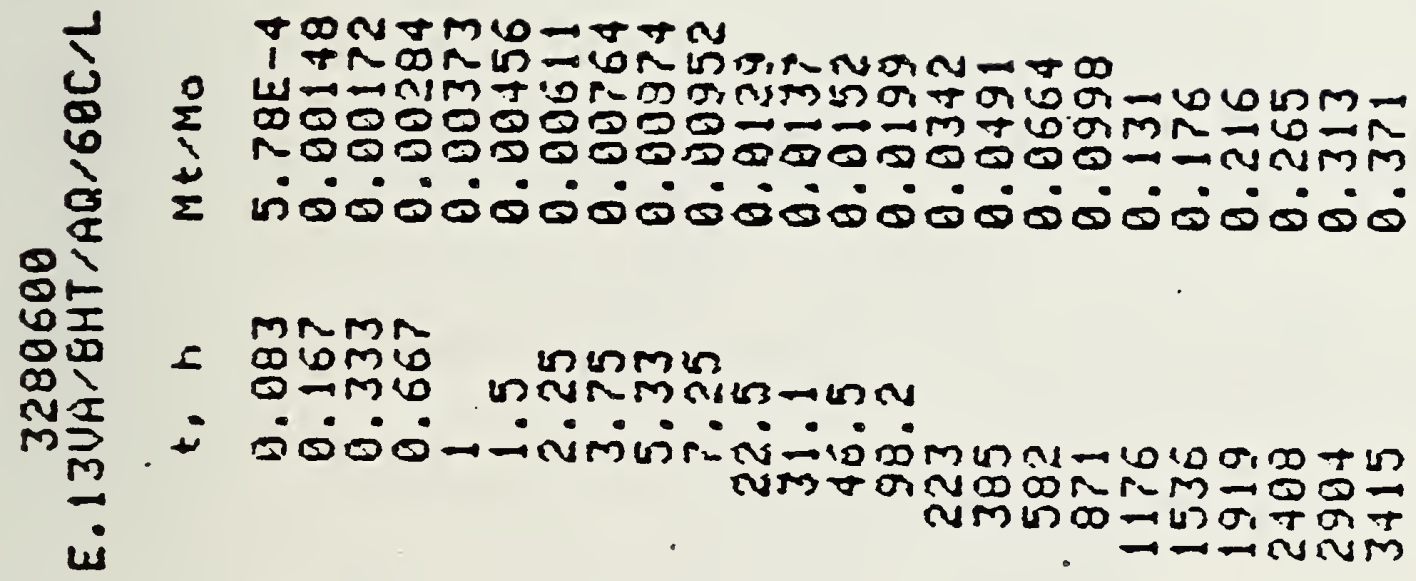

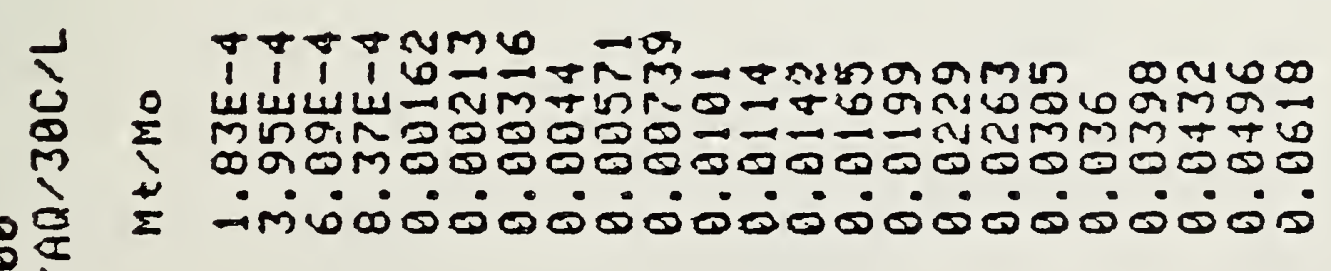

MMNMN

I MDOOMLMN US

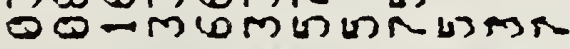

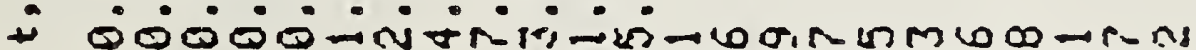

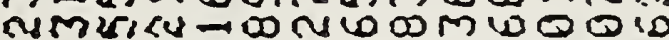
जलm
} 
NBS.114A (AEV. Z.AC)

U.3. OEPT. OF SOMM.

BIBLIOGRAPHIC DATA

SHEET (See instruc:ions)

1. PUBLICATION OR REPORT NO.

NBSIR $82-2472$
2. Performing Orzan. Report Nof 3. Puolication Date

March 1982

4. TITLE AND SUBTITLE

Migration of Low Molecular Additives in Polyolefins and Copolymers

5. AUTHOR(S)

5. S. Chang, G. A. Senich, and L. E. Smith

6. PERFORMING ORGANIZATION (If jOint or Other than NBS. see instruc:ions)

7. Contracer Grane No.

MATIONAL BUREAU OF STANDARDS

DEPARTMEXT OF COMMERCE

WASHINGTOH, D.C. 20234

3. Tyoe of Report \& Period Covere

9. SPONSORING ORGANIZATION NAME AND COMPLETE AOORESS (Stret. CITY. SLOtR, ZIP)

10. SUPPLEMENTARY NOTES

$\begin{array}{rr}\text { Other Related Publications } 73-1499 & 30-1999 \\ 79-1598 & 81-2264 \\ 79-1779 & 31-2314\end{array}$

Document descilbes a comouter program: SF-185. FiPS Sofiware Summary. is attarhed.

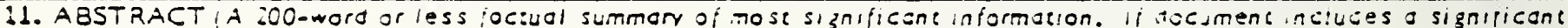
bibliograony or literoture survey, mention it here)

Food packaging is an insaparable part of modern life. Any substance that migrates from the packaging materiai into foods is vieived as indirect food additives. In connection witn toxicological knowiedge, it is important to know the amount of such indirect food additives expected to be present in the food during storage and processing. This program, sponsored by the Bureau of Foods of the food and Orug idministration is to proviue theoretical models, reliable data base, methodology to study the migration pheriomena and to provide reasonabie worst-case estimates for tne concentrations oi the indirect additives in food. In this final report we presant the results of approximately 250 completed nigration experimenss based on radiotracer techniques on the migration of low molecular weight hyoracarbons and antioxidants from polyethylene, polyoropylene and ethylene-vinyl acetace cocolymers.

Results of a study for the detemination of relative difiusion coefficients of several probe molecules in the polvechylene melt by inyerse gas chromatography are also presanted. 3ased on these studies, etharo! aDcears to be a far more reasonadie food-oil stimulating soivent than n-neptane. itner pure or mixed triglycerides may also be considered as food-oil simulating solvents, however they may pose the same analytical difiticuities as inat oi the food oil itself.

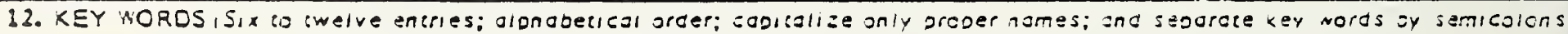
antioxidants; diffusion; ethylene-vinyl acetate copolymers; iood sackaging; inverse gas chromatography; migration; ol igomers; polyethylene; polyoropylene; radintracor

13. AVAILABILITY

X Unlimited

For Official Distribucion. Do Not Release to NTIS

Order From Suderintendent of Documents, U.S. Government Peinting Oifics, Nashington, O.C. 20402.

14. NO. OF

PRINTED PAGE

110

Xl Order From National Technical Information Service (NTIS), Soringfield. VA. 22161

15. Price 

